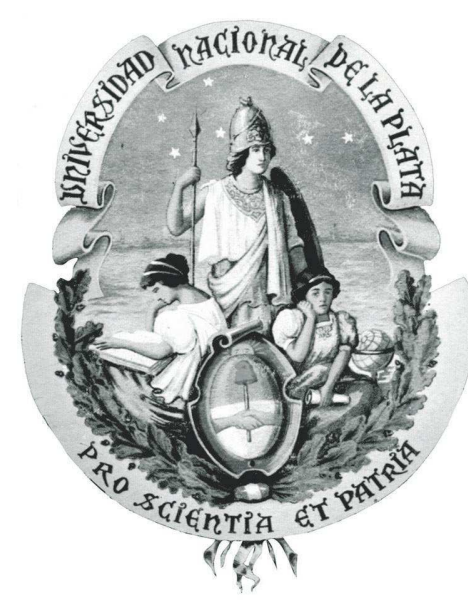

UNIVERSIDAD NACIONAL DE LA PLATA

Facultad de Ciencias Exactas

Departamento de Física

\title{
Aplicaciones de la Conjetura AdS/CFT
}

Tesis presentada para optar al título de Doctor de la Universidad de La Plata en el área Ciencias Físicas

\section{Lic. Raúl E. Arias}

Director de tesis: Dr. Guillermo Silva

Lugar de trabajo: Departamento de Física, Facultad de Ciencias Exactas, UNLP

La Plata, Febrero de 2013 


\section{Resumen}

En esta tesis se estudian las aplicaciones de la conjetura gravedad/gauge (AdS/CFT) al cálculo de funciones de correlación y lazos de Wilson en teorías de gauge mínimamente supersimétricas conformes y no conformes. También se desarrolla un ejemplo de la aplicación de la conjetura al estudio de la termodinámica de un sistema superconductor de interés en el contexto de materia condensada.

En el capítulo 1 se introducen los conceptos fundamentales de la correspondencia AdS/CFT, en el capítulo 2 se extenderá la prescripción de Gubser-Klebanov-Polyakov-Witten (GKPW) a duales gravitatorios con más de un borde.

En el capítulo 3 se discutirá y aplicará la prescripción de Maldacena-Rey para calcular el potencial quark-antiquark y el monopolo-antimonopolo en diferentes fondos gravitatorios duales a teorías conformes y no conformes. En particular, se analizará la estabilidad de las soluciones clásicas frente a pequeñas fluctuaciones.

En el capítulo 4 se investigarán las propiedades termodinámicas y de la entropía de entrelazamiento en sistemas superconductores del tipo $p$ y $p+i p$ en $2+1$ dimensiones a través de sus modelos duales gravitatorios. Se estudiará el límite en que el número de grados de libertad cargados es comparable con el número de grados de libertad totales, lo cual corresponde a considerar en la descripción gravitatoria la deformación de la geometría debida a un campo de gauge no Abeliano. 


\section{Agradecimientos}

En primer lugar quiero agradecer a mi director, colega y amigo Guillermo Silva. A mis amigos de la facultad Ale, Blai, Carlos L, Carlos C, Colo, Diego C, Diego M, Diego R, Gastón, Gianni, Juan, Marcelo, Mariela, Mauricio, Nacho, Nico, Pablo P, Pablo RP y Walter. Mención especial para mi compañero de oficina Diego T por los gratos momentos compartidos. También agradezco a mi familia por el apoyo constante y especialmente a Romi, a quien le dedico esta tesis. 


\section{Índice general}

1. Introducción $\quad 5$

1.1. Correspondencia Gauge/Gravedad . . . . . . . . . . . . . . . . . 5

1.1.1. La Correspondencia . . . . . . . . . . . . . . . . . 6

1.1.2. Funciones de Correlación . . . . . . . . . . . . . . . . . . 9

1.1.3. Lazos de Wilson . . . . . . . . . . . . . . . . . . . . 15

1.2. Aplicaciones a materia condensada . . . . . . . . . . . . . . . 22

2. $A d S$ Lorentziano, Agujeros de Gusano y Holografía 26

2.1. Prescripción GKPW con un solo borde asintótico f . . . . . . . . . . 28

2.2. Prescripción GKPW para agujeros de gusano Lorentzianos . . . . . . . . . . . . . 35

2.3. Entrelazamiento vs. Acoplamiento _. . . . . . . . . . . . . . . . . . . 41

2.4. Conclusiones . . . . . . . . . . . . . . . . . . . . 45

$\begin{array}{ll}\text { 3. Estabilidad de lazos de Wilson } & 48\end{array}$

3.1. Soluciones de Cuerda y Lazos de Wilson . . . . . . . . . . . . . . . . . . 50

3.1.1. Cuerdas estáticas . . . . . . . . . . . . . . . . 50

3.1.2. Análisis de Estabilidad de embeddings de cuerdas clásicas . . . . . . . . . 54

3.2. Fondos Gravitatorios . . . . . . . . . . . . . . . . . . . . 58

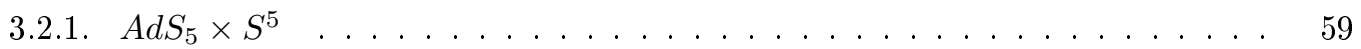

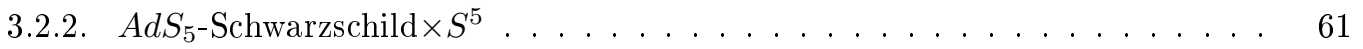

3.2.3. Maldacena-Núñez . . . . . . . . . . . . . . . . . . . . . . . . . 63

3.2.4. Klebanov-Strassler . . . . . . . . . . . . . . . . . . . 66

3.2.5. Maldacena-Núñez generalizado . . . . . . . . . . . . . . . 68

3.3. Análisis de Estabilidad . . . . . . . . . . . . . . . . . . . . . . . 71

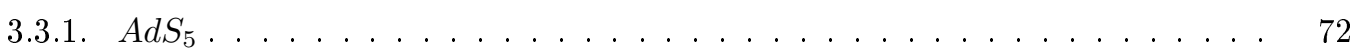

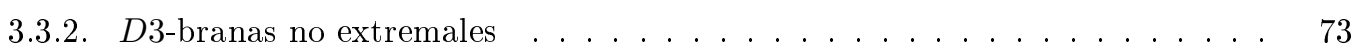

3.3.3. Maldacena-Núñez . . . . . . . . . . . . . . . . . . . . . . . . . . . 74 
3.3.4. Klebanov-Strassler . . . . . . . . . . . . . . . . . . . . . . . . . 75

3.3.5. Maldacena-Núñez generalizada . . . . . . . . . . . . . . 75

3.4. Análisis de Potenciales de Schrödinger . . . . . . . . . . . . . . . . . 77

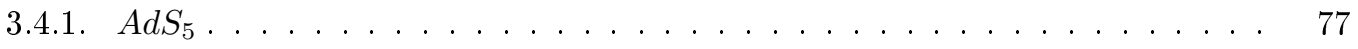

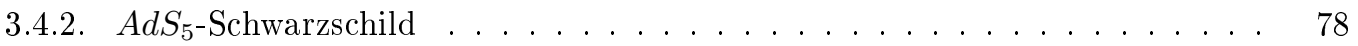

3.4.3. Maldacena-Núñez . . . . . . . . . . . . . . . . . . . . . . . . 79

3.4.4. Klebanov-Strassler . . . . . . . . . . . . . . . . . . . . . . . . 80

3.4.5. Maldacena-Núñez Generalizado . . . . . . . . . . . . . . . . . . . . . . 81

3.5. Lazo de 't Hooft . . . . . . . . . . . . . . . . . . . . . . . . . . 82

3.5.1. Maldacena-Núñez . . . . . . . . . . . . . . . . . . . . . . 82

3.5.2. Klebanov-Strassler . . . . . . . . . . . . . . . . . . 84

3.6. Fluctuaciones del Lazo de Wilson en representaciones de orden más alto . . . . . . 85

3.6.1. Acción general para D-branas . . . . . . . . . . . . . . . . 85

3.6.2. Lazo de Wilson-’t Hooft y sus fluctuaciones . . . . . . . . . . . . . . . 86

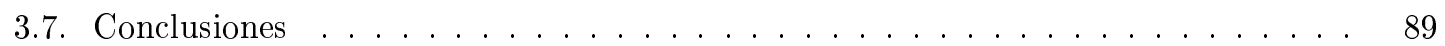

4. Superconductores Holográficos $\quad 92$

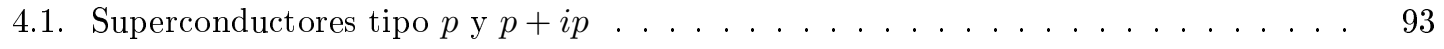

4.1.1. Superconductor tipo $p$ en $2+1$ dimensiones . . . . . . . . . . . . . . 94

4.1.2. Superconductores tipo $p+i p \ldots \ldots \ldots$. . . . . . . . . . 100

4.2. Entropía de entrelazamiento . . . . . . . . . . . . . . . . . . . . . 104

4.2.1. Conclusiones . . . . . . . . . . . . . . . . . . . . 107

$\begin{array}{lr}\text { 5. Conclusiones } & 109\end{array}$

$\begin{array}{ll}\text { Apéndices } & 111\end{array}$

A. Lazos de Wilson $\quad 111$

A.1. De Sturm-Liouville a Schrödinger . . . . . . . . . . . . . . . . . . . . . . . 111

A.2. Espectro para fluctuaciones transversales en $A d S_{5} \times S^{5} \ldots \ldots \ldots \ldots$. . . . . 112

A.3. Fluctuaciones fermiónicas del operador de Wilson-'t Hooft . . . . . . . . . . . . . 113

$\begin{array}{ll}\text { Bibliografía } & 117\end{array}$ 


\section{Capítulo 1}

\section{Introducción}

\subsection{Correspondencia Gauge/Gravedad}

En 1997 Maldacena [1] conjeturó la realización de una antigua idea de 't Hooft [2] en un modelo concreto: la teoría de cuerdas IIB formulada sobre $A d S_{5} \times S^{5}$ es exactamente dual al límite $N \gg 1$ de la fase conforme de la teoría supersimétrica de Yang-Mills (SYM) $\mathcal{N}=4$ con grupo de gauge $S U(N)$. Esta idea se conoce como conjetura de dualidad $A d S / C F T$ puesto que la teoría de gauge es conforme. Si bien la conjetura no ha sido probada, la dualidad ha superado un número importante de chequeos (ver [3]). En particular, la conjetura establece que la teoría de gauge dual a un cierto fondo gravitatorio está definida sobre el borde asintótico de dicho espacio-tiempo $[4,5]$, ver sección 1.1.2. La dualidad entre estas dos teorías tan disímiles se encontró analizando las descripciones complementarias en términos de cuerdas abiertas y cerradas de un sistema de $N$ D3-branas en el límite de horizonte cercano, ver sección 1.1.1. Puesto que la teoría de bajas energías de la teoría de cuerdas es supergravedad, gran parte de las aplicaciones se centran en el estudio de la supergravedad IIB sobre el fondo $A d S_{5} \times S^{5}$. Más concretamente, la supergravedad IIB describe el régimen "planar/no perturbativo de la teoría $\mathcal{N}=4$ SYM. Siguiendo estas ideas, muchos trabajos se han dedicado a la extensión a teorías con menor número de supersimetrías $[6,7]$ y la introducción de quarks (materia en la fundamental) [8]. Por otro lado la conjetura es sorprendente desde el punto de vista que relaciona dos teorías totalmente diferentes definidas sobre espacio-tiempos de dimensiones distintas.

¿ Por qué es importante esta conjetura? La respuesta está relacionada con la siguiente propiedad: la dualidad relaciona el regimen de acoplamiento fuerte de una teoría de gauge con el regimen semiclásico de la teoría de cuerdas IIB. Esto significa que usando la conjetura podemos derivar propiedades de la teoría de gauge en el regimen de acoplamiento fuerte (el cual es un límite difícil de estudiar con técnicas usuales de teorías de campos) estudiando una teoría de cuerdas semiclásica 
en su regimen de acoplamiento débil.

En este capítulo se introducirán los conceptos fundamentales de la correspondencia AdS/CFT señalando más detenidamente aquellos aspectos que se desarrollarán a lo largo de esta tesis.

\subsubsection{La Correspondencia}

La conjetura de Maldacena, o AdS/CFT, fue propuesta en [1] y establece la existencia de una equivalencia entre una teoría de gauge y una teoría de cuerdas $[9,10]$. La correspondencia se infiere a partir de dos descripciones alternativas de un sistema de D-branas [11].

Consideremos una pila de $N$ D3-branas (coincidentes) en un espacio-tiempo de Minkowski 10dimensional, es decir, no consideramos la backreaction de las branas sobre el espacio-tiempo, lo que equivale a tomar $g_{s} N \ll 1$, donde $g_{s}$ es la constante de acoplamiento de la cuerda. El espectro de bajas energías de las cuerdas abiertas que terminan sobre el volumen 4-dimensional de las branas es descripto por partículas puntuales no masivas que componen un multiplete supersimétrico $\mathcal{N}=4$ super Yang-Mills y que transforman bajo el grupo de gauge $S U(N)$. Esta teoría de campos goza de invarianza conforme aún a nivel cuántico. Esquemáticamente la acción de bajas energías que describe el sistema es

$$
S_{\text {open }}=S_{\text {brane }}+S_{\text {bulk }}+S_{\text {int }} \approx S_{\mathcal{N}=4}+S_{\text {bulk flat }}+S_{\text {int }}, \quad g_{s} N \ll 1
$$

Aquí $S_{b u l k}$ es la acción de supergravedad en 10 dimensiones e involucra modos no masivos de cuerdas cerradas que viven en el bulk (espacio plano). $S_{\text {brane }}$ da cuenta de los modos no masivos del espectro de cuerdas abiertas y su límite de bajas energías es el Lagrangiano de $\mathcal{N}=4 \mathrm{SYM}$. El termino $S_{\text {int }}$ representa el acoplamiento entre modos de las cuerdas abiertas y las cerradas, a bajas energías se obtiene que $S_{\text {int }} \rightarrow 0$ [12]. En el límite en que $\lambda=g_{s} N \gg 1$ debemos considerar la deformación al espacio-tiempo debida a las branas. La dinámica del sistema queda descripta simplemente por cuerdas cerradas en la geometría

$$
\begin{aligned}
d s^{2}= & H(r)^{-1 / 2}\left(-d t^{2}+d x_{3}^{2}\right)+H(r)^{1 / 2}\left(d r^{2}+r^{2} d \Omega_{5}^{2}\right) \\
& H(r)=1+\frac{N G_{N}}{r^{4}}, \quad G_{N}=g_{s} \alpha^{\prime}
\end{aligned}
$$

La acción que describe este sistema es

$$
S_{\text {close }}=S_{\text {bulk throat }}+S_{\text {bulk flat }}+S_{\text {int }}, \quad g_{s} N \gg 1
$$

en donde $S_{\text {bulk flat }}$ da cuenta de los modos no masivos de cuerdas cerradas en la región $r \rightarrow \infty$ (espacio plano) de (1.1) y $S_{\text {bulk throat }}$ denota la acción para todos los modos (masivos y no masivos) de cuerdas en la región descripta por $r \rightarrow 0$ en la geometría (1.1), es decir, en $\operatorname{AdS}_{5} \times S^{5}$. Notemos 
que debido al corrimiento al rojo gravitatorio modos masivos de cuerdas en la región que interpola entre Mikowski en 10 dimensiones y $\mathrm{AdS}_{5} \times S^{5}$ (throat) son de baja energía con respecto a un observador en el infinito, y deben ser tenidos en cuenta al tomar el límite de bajas energías. Por lo tanto $S_{\text {bulk throat }} \equiv S_{\text {strings } A d S_{5} \times S^{5}}$. $S_{\text {int }}$ representa el acoplo entre modos que viven en el throat y modos que viven en el espacio plano. De nuevo, a bajas energías $S_{i n t} \rightarrow 0$. Pero ahora, nótese que hay dos descripciones para el mismo sistema y por lo tanto $S_{\text {open }}$ y $S_{\text {close }}$ deben ser equivalentes. Luego, comparando $S_{\text {open }}$ y $S_{\text {close }}$ se obtiene que

$$
S_{\mathcal{N}=4}=S_{\text {strings } A d S_{5} \times S^{5}}
$$

En resumen, la correspondencia AdS/CFT conjetura la equivalencia entre dos teorías:

- $\mathcal{N}=4$ super Yang-Mills con grupo de gauge $S U(N)$ (generada por los modos no masivos de cuerdas abiertas) para $N \gg 1$

- teoría de supercuerdas IIB sobre un fondo $\mathrm{AdS}_{5} \times S^{5}$ con un número entero del flujo de la 5 -forma de Ramond-Ramond, $N=\int_{S^{5}} F_{5}$, (generada por toda la torre de estados de la teoría de cuerdas sobre AdS).

El diccionario de la correspondencia relaciona los dos parámetros de la teoría de campos, es decir la constante de acoplamiento $g_{Y M}$ y el rango del grupo de gauge $N$, con la constante de acoplamiento de la teoría de cuerdas $g_{s}$ y el tamaño de la cuerda $l_{s}=\sqrt{\alpha^{\prime}}$ mediante [1]

$$
g_{Y M}^{2}=g_{s}, \quad\left(\frac{R}{l_{s}}\right)^{4}=4 \pi g_{Y M}^{2} N=4 \pi \lambda
$$

en donde $R$ es el tamaño de AdS y $\lambda=g_{Y M}^{2} N$ es la constante de 't Hooft. Estas ecuaciones indican que dependiendo de la elección de los parámetros podemos estudiar el régimen de acoplamiento débil de la teoría de gauge o de la teoría de cuerdas, pero nunca será simultáneo en ambas teorías. Se observa de (1.2) que en el límite en el cual $\lambda \rightarrow \infty$ (el tamaño de la cuerda es despreciable frente al tamaño de AdS), corresponde al regimen semiclásico de cuerdas, es decir una teoría de supergravedad. La tensión efectiva de la cuerda $T_{e f}=\sqrt{\lambda} \rightarrow \infty$ y las cuerdas son puntuales. Nótese que en este límite la teoría de gauge se encuentra fuertemente acoplada y por lo tanto no es posible estudiarla perturbativamente. El régimen opuesto (cuando el tamaño de la cuerda es comparable con el tamaño del espacio) nos dice que debemos analizar la teoría cuántica de cuerdas en AdS a fin de hacer predicciones sobre el límite de acoplamiento débil de la teoría de campos. Luego, la correspondencia puede ser aplicada en dos direcciones, podemos usar la teoría de cuerdas en su límite semiclásico para aprender sobre las propiedades de una teoría de gauge fuertemente acoplada ó podemos estudiar una teoría de campos en su regimen perturbativo para entender la teoría cuántica de cuerdas sobre fondos no triviales como lo es por ejemplo AdS. 
Un primer chequeo de la conjetura es la correspondencia entre el grupo de isometrías de la teoría de cuerdas y el grupo conforme de $\mathcal{N}=4$. En particular, el grupo de isometrías de $\mathrm{AdS}_{5}$ coincide con el grupo conforme en 4 dimensiones $S O(4,2)$. Por otro lado, la teoría $\mathcal{N}=4$ tiene una simetría global adicional, además de las de Poincaré y supersimetría, dada por el grupo $S U(4)_{\mathcal{R}} \sim S O(6)$ y denominada simetría $\mathcal{R}$. No es difícil ver entonces que el origen del espacio 10-dimensional en donde vive la teoría de cuerdas debe ser el producto de un espacio AdS y una esfera 5-dimensional $S^{5}$. En otras palabras, cada vez que la teoría de gauge sea una teoría conforme (a nivel cuántico) debemos tener un espacio AdS en su dual gravitatorio. Por otra parte cualquier grupo de simetría adicional en la teoría SYM se relaciona con la simetría del espacio interno $\mathcal{M}$, la teoría de cuerdas dual queda definida en $\operatorname{AdS} \times \mathcal{M}$.

La conjetura AdS/CFT implica una identificación entre la coordenada radial $r$ en la solución de supergravedad y la escala de energía de la teoría de campos dual a ella. Esta identificación surge de analizar cómo se encuentra realizado el operador de dilataciones $D \subset \operatorname{Conf}\left(\mathbb{R}^{3,1}\right)=\operatorname{so}(2,4)$ en la solución de gravedad. El resultado es que una dilatación $x_{i} \rightarrow \lambda x_{i}$ en la teoría de gauge se corresponde en AdS: $d s^{2}=d r^{2}+e^{2 r / R}\left(d x_{i}\right)^{2}$ con la isometría

$$
\begin{array}{cc}
\qquad x_{i} \rightarrow \Lambda x_{i} & x_{i} \rightarrow \lambda x_{i}, \quad r \rightarrow r-R \log \lambda \\
\text { teoria de gauge } & \text { gravedad }
\end{array}
$$

El borde del AdS $(r \rightarrow \infty)$ se asocia con el regimen ultravioleta (UV) de la teoría de campos conforme (CFT), mientras que el horizonte del AdS $(r \rightarrow 0)$ se corresponde con el régimen infrarrojo (IR). Esta asociación es más que una identificación formal como veremos al calcular lazos de Wilson: cálculos de valores de expectación de vacío (VEV) de operadores en la teoría de gauge con una escala característica $\mu$, se encuentran dominados a nivel semiclásico por contribuciones provenientes de la región $r=R \log \mu$ de la geometría (ver [3] para mas ejemplos y referencias).

Un cambio de escala en una CFT es irrelevante físicamente. Sin embargo, en una teoría no conforme las constantes de acoplamiento cambian con la escala. La no invarianza conforme de la teoría cuántica de campos se manifiesta en el lado gravitatorio en la ausencia de una simetría que relacione diferentes valores de la dirección radial. Esto sugiere interpretar la dependencia de los acoplamientos con la escala en términos de una dependencia específica para los campos de la solución de gravedad. Esta interpretación en la teoría de campos funciona razonablemente a nivel cualitativo, sin embargo, la identificación cuantitativa del radio con la escala de energía puede ser difícil de hallar. Una posibilidad para identificar la relación radio/energía es considerar el factor de enrollamiento $Z(r)$ que multiplica el hiperplano $3+1$ en la métrica $d s^{2}=Z(r) d x_{i} d x^{i}+\ldots$, puesto que $Z(r)$ es el factor que relaciona las energías de dos observadores ubicados en puntos distintos del espacio transverso mediante: $Z\left(r^{\prime}\right)^{-1 / 2} E^{\prime}=Z(r)^{-1 / 2} E[3]$. 


\section{Extensión a teorías no conformes y menos supersimétricas}

Hasta acá se discutió la conjetura originalmente postulada por Maldacena, en donde la teoría de campos es invariante conforme y supersimétrica. Una pregunta natural es cómo encontrar duales gravitatorios para teorías de gauge más realistas, es decir, con menos (o ninguna) supersimetrías y que no gocen de invarianza conforme. Como veremos a continuación, resulta problemático describir teorías realistas mediante supergravedad (ver [13]).

Las razones son las siguientes: existen conceptualmente dos maneras de romper la simetría conforme: (i) comenzar con una teoría conforme para la cual conocemos su dual gravitatorio y deformarla mediante operadores relevantes o marginales que rompen supersimetría e invarianza conforme, técnicamente esto corresponde a encender campos en la solución de gravedad, ó (ii) comenzar con una configuración donde la supersimetría y la invarianza conforme se encuentran rotas (las soluciones discutidas en el capítulo 3 pertenecen a esta segunda categoría). En el primer caso, una manera posible de obtener "SYM"pura sería agregar una deformación con parámetro de masa $M$ a los campos escalares de $\mathcal{N}=4$. Ahora bien, el parámetro de masa $M$ inducirá una escala dimensional $\Lambda \sim M e^{-1 / g_{Y M}^{2} N}$. El límite en el cual la deformación da origen a SYM $(\Lambda$ finito) es $M \rightarrow \infty, \lambda=g_{Y M}^{2} N \ll 1$ con $\lambda$ fijo. Sin embargo, como discutimos en la introducción, la aproximación de supergravedad es confiable en el límite opuesto $\lambda \gg 1$. De manera que, en términos generales, la descripción de bajas energías de SYM pura requiere el conocimiento completo del espectro de la teoría de cuerdas. El segundo caso (ii) enumerado arriba corresponde al de enrollamiento de branas. La característica principal y recurrente de este tipo de soluciones es que los grados de libertad de la teoría de gauge se encuentran entrelazados con los modos de KaluzaKlein (modos KK) de la variedad transversa y no es posible desacoplarlos. Los modos KK entran en juego a una escala de energía que es inversamente proporcional al tamaño de la variedad, y el inconveniente en este tipo de soluciones es que este tamaño de la variedad compacta es comparable con la escala en la que los fenómenos no perturbativos son relevantes (confinamiento, ruptura de simetría quiral,...). En resumen, soluciones clásicas de supergravedad describen apropiadamente teorías que no son puramente teorías de Yang-Mills, sino que contienen un número infinito de campos adicionales. Se espera que el dual holográfico en el límite de acoplamiento débil de QCD sea un modelo de cuerdas fuertemente acoplado.

\subsubsection{Funciones de Correlación}

La conjetura fue definida más precisamente luego de los trabajos de Witten [5] y Polyakov et. al. [4], en donde se propuso una técnica de cálculo para la relación entre valores de expectación de operadores de la CFT y la función de partición de la teoría de cuerdas. Es el propósito de esta 
sección analizar en detalle dicha relación.

Para definir la correspondencia necesitamos una relación unívoca entre los observables de ambas teorías y una prescripción para comparar cantidades físicas. Desde el punto de vista de la teoría 4-dimensional, todo operador $\mathcal{O}$ invariante de gauge puede asociarse a una fuente $h$

$$
L_{C F T}+\int d^{4} x h \mathcal{O}
$$

en donde $L_{C F T}$ es el Lagrangiano de la teoría 4-dimensional. Usualmente se define la funcional generatriz, $W(h)$, de funciones de correlación del operador $\mathcal{O}$ a partir de

$$
e^{-W(h)}=\left\langle e^{-\int h \mathcal{O}}\right\rangle_{Q F T}
$$

y el valor de expectación de los operadores se escribe como

$$
\langle\mathcal{O} \ldots \mathcal{O}\rangle_{c}=(-1)^{n}\left[\frac{\delta^{n} W}{\delta h^{n}}\right]_{h=0} .
$$

Un aspecto básico de la conjetura es la siguiente afirmación: todo operador invariante de gauge de la teoría cuántica se relaciona con un campo $h$ en AdS (ambos deben tener los mismos números cuánticos). La correspondencia propone identificar a la fuente del operador $(h(x))$ con el valor en el borde de un campo 5-dimensional $h(x, z)$ ( $z$ es la coordenada radial de AdS). Para cada posible fuente externa en la CFT hay un campo 5 -dimensional $h(x, z)$. Con esto podemos entender la ecuación fundamental de la conjetura AdS/CFT

$$
e^{W(h)}=\left\langle e^{\int h O}\right\rangle_{Q F T}=\mathcal{Z}_{\text {strings en } \operatorname{AdS}}\left[h(x, z=\text { borde })=h_{0}\right] .
$$

En el lado izquierdo de esta ecuación tenemos una funcional que depende de una fuente arbitraria 4-dimensional (QFT en presencia de fuente externa) y del lado derecho tenemos la función de partición de la teoría de cuerdas con particulares condiciones de contorno para los campos definidos en el espacio AdS. La función de partición es un objeto complicado de calcular. En el límite semiclásico de cuerdas $(\lambda \gg 1)$ se aproxima como

$$
\mathcal{Z}_{\text {strings en } \operatorname{AdS}}[h(x, z=\text { borde })] \sim e^{S_{\text {sugra }}\left[h_{0}\right]}, \quad \lambda \gg 1
$$

En el lado derecho la acción de supergravedad 5-dimensional se encuentra evaluada sobre la solución de sus ecuaciones de movimiento que tiene por valor de borde $h_{0}(x)$. En resumen, (1.7) y (1.8) prescriben que el valor de borde de campos de supergravedad actúa como una fuente para operadores de la teoría de gauge dual.

Algunos comentarios adicionales al respecto de las ecuaciones (1.7) y (1.8):

- Las ecuaciones de movimiento en AdS son ecuaciones diferenciales de segundo orden y por lo tanto vamos a necesitar estudiar las condiciones de borde para determinar una solución 
única. La asociación no es tan simple como $h(x$, borde $)=h_{0}(x)$, veremos más adelante que la condición adecuada es pedir $h(x, z) \sim f(z) h(x)$. Por otro lado, la segunda condición de borde depende fuertemente de la signatura de AdS. Si se está trabajando con AdS Euclídeo pedir regularidad en el interior del espacio determina la solución unívocamente. Se verá en el capitulo 2 que en signatura Lorentziana pedir regularidad en el horizonte no es suficiente para tener una solución única, se requiere información adicional en el bulk relativa a los estados entrantes y salientes en las tapas del cilindro de AdS [14, 15, 16, 17] (ver figura 1.1 y 1.2).

- El campo $h$ que se acopla al operador $\mathcal{O}$ usualmente puede identificarse mediante simetrías debido a que ambos deben tener los mismos números cuánticos. En particular, para el caso de corrientes conservadas los acoplamientos son conocidos

$$
L_{C F T}+\int d^{4} x \sqrt{g}\left(g_{\mu \nu} T^{\mu \nu}+J_{\mu} A^{\mu}+\phi \operatorname{Tr}\left(F_{\mu \nu} F^{\mu \nu}\right)+\ldots\right) .
$$

El operador asociado al gravitón se corresponde con el tensor de energía-momento y el asociado con un campo de gauge en AdS a una corriente (global) conservada. Nótese que la conservación de $T_{\mu \nu}$ ó de $J_{\mu}$ se asocia con una invariancia de gauge al nivel de las fuentes (esto significa que $W\left(g_{\mu \nu}, A_{\mu}\right)$ es una funcional invariante de gauge frente a $A_{\mu}$ y $\left.g_{\mu \nu}\right)$. Este es un hecho general de AdS/CFT, simetrías de gauge en el bulk son simetrías globales en la teoría del borde.

En lo que sigue de la sección voy a reproducir un ejemplo canónico con el objetivo de entender el poder de la expresión (1.8). Consideremos el caso de un campo escalar masivo en $\operatorname{AdS}_{d+1}[4,5]$, el cual es dual a un operador $\mathcal{O}$ en la CFT dual. La métrica de $\operatorname{AdS}_{d+1}$ en coordenadas de Poincaré se escribe

$$
d s^{2}=R^{2} \frac{d x^{\mu} d x_{\mu}+d z^{2}}{z^{2}} \equiv g_{A B} d x^{A} d x^{B}, \quad A=0, \ldots, d, \quad x^{A}=\left(z, x^{\mu}\right)
$$

En estas coordenadas el borde de AdS se sitúa en $z=0$ y la geometría del mismo es la del espacio de Minkowski. La figura 1.1 muestra la topología en forma de esfera de la métrica (1.10) en coordenadas Euclídeas, por otro lado la figura 1.2 muestra la topología cilíndrica de AdS en signatura Lorentziana y la región cubierta por las coordenadas (1.10) (Poincaré patch).

Dado un campo escalar $\phi\left(z, x^{\mu}\right)$, su acción en un espacio curvo se escribe

$$
S \sim \int d^{d+1} x \sqrt{g}\left(g^{A B} \partial_{A} \phi \partial_{B} \phi+m^{2} \phi^{2}\right) .
$$

Integrando por partes y usando el teorema de Gauss tenemos

$$
S \sim \int_{\partial A d S} d^{d} x \sqrt{g} g^{z B} \phi \partial_{B} \phi+\int d^{d+1} x \sqrt{g} \phi\left(-\square+m^{2}\right) \phi,
$$




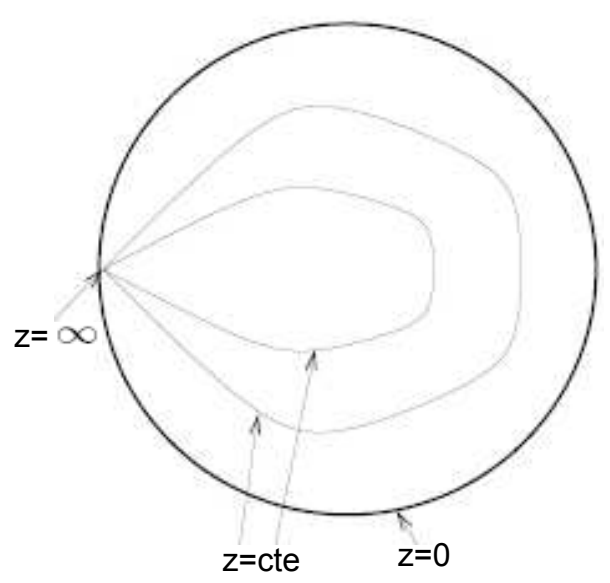

Figura 1.1: AdS en coordenadas de Poincaré con signatura Euclídea. La topología en forma de esfera se obtiene de mapear todos los puntos del infinito a un solo punto. Las coordenadas de Poincaré cubren toda la variedad.

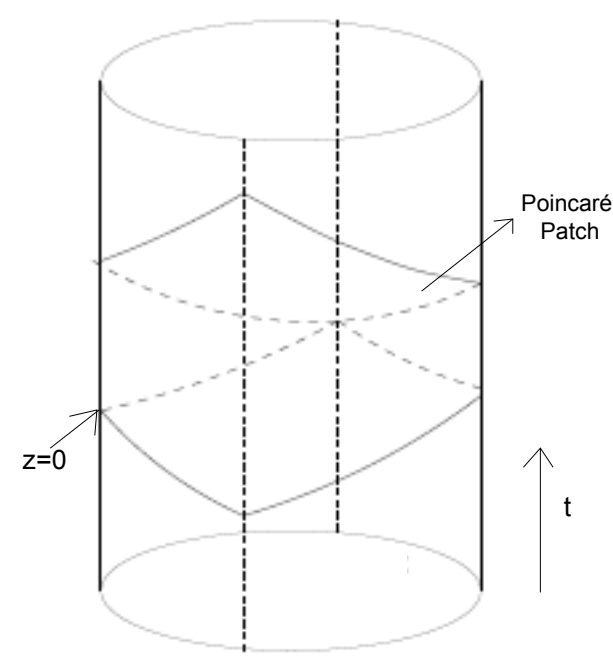

Figura 1.2: Topología de AdS en signatura Lorentziana. La región denominada Poincaré patch es la región del espacio cubierta por las coordenadas de Poincaré (1.10).

en donde $\square \phi=\frac{1}{\sqrt{g}} \partial_{A}\left(\sqrt{g} g^{A B} \partial_{B}\right) \phi$. Nótese que el segundo término es la ecuación de Klein-Gordon para un campo escalar masivo en un espacio curvo y, por lo tanto, la acción on-shell está dada para el caso libre simplemente por el primer término de (1.12).

Nuestra intención ahora es resolver la ecuación de movimiento para $\phi,\left(\square+m^{2}\right) \phi=0$ con condición de borde " $\phi(0, x)=\phi_{0}(x) "$. Descomponiendo al campo escalar en modos de Fourier

$$
\phi\left(z, x^{\mu}\right)=e^{i k_{\mu} x^{\mu}} f_{k}(z), \quad k_{\mu} x^{\mu}=-\omega t+\vec{k} \cdot \vec{x}
$$

y usando el hecho de que la métrica solo depende de $z$, la ecuación de movimiento para el campo escalar queda

$$
0=\frac{1}{R^{2}}\left(z^{2} k^{2}-z^{d+1} \partial_{z}\left(z^{-d+1} \partial_{z}\right)+m^{2} R^{2}\right) f_{k}(z), \quad k^{2}=\vec{k}^{2}-\omega^{2} .
$$

En el límite $z \rightarrow 0$ la solución asintótica de la ecuación de movimiento es $f_{k} \sim z^{\Delta}$, en donde $\Delta$ queda definida por

$$
m^{2} R^{2}=\Delta(\Delta-d)
$$

Esta ecuación tiene dos soluciones, genéricamente una positiva $\Delta_{+}$y otra negativa $\Delta_{-} . \Delta_{+}$se identifica con la dimensión conforme del campo dual $\mathcal{O}$. Las coordenadas de Poincaré no cubren toda (la variedad) AdS en el caso Lorentziano, solo una parte (ver figura 1.2). De manera que al definir el problema de $\phi$ en el interior debemos dar condiciones de contorno no solo en $z=0$, sino 
también en el horizonte $(z \rightarrow \infty)$. Las dos soluciones linealmente independientes de la ecuación diferencial (1.14) cerca del borde son

$$
\phi \sim \phi_{+} z^{\Delta_{+}}+\phi_{-} z^{\Delta_{-}},
$$

siendo $\Delta_{ \pm}=\frac{d}{2} \pm \sqrt{\left(\frac{d}{2}\right)^{2}+m^{2} R^{2}}=\frac{d}{2} \pm \nu$ las raíces de la ecuación (1.15) y $\phi_{ \pm}(k)$ dos constantes. El primer término $\phi_{+}(k)$ da origen a una solución normalizable en el sentido de que la expresión

$$
\int d z \sqrt{g}|\phi|^{2}<\infty
$$

El coeficiente $\phi_{-}(k)$ corresponde a una solución no normalizable.

Debido al carácter singular del borde de AdS $(z=0)$ es conveniente introducir una regularización en $z=\epsilon$ y tomar $\epsilon \rightarrow 0$ al final de los cálculos como veremos a continuación. La condición de contorno es entonces

$$
\left.\phi\left(z, x^{\mu}\right)\right|_{z=\epsilon} \rightarrow \epsilon^{\Delta_{-}} \phi_{-}^{r e n}\left(x^{\mu}\right) .
$$

La función $\phi_{-}^{r e n}$ se identifica con la fuente del operador $\mathcal{O}$.

Hay varias observaciones que mencionar con respecto a la ecuación (1.15):

- Heurísticamente puede demostrarse que $\mathcal{O}(\epsilon, x) \sim \epsilon^{\Delta_{+}} \mathcal{O}^{r e n}(x)$ y por lo tanto, la dimensión de escala de $\mathcal{O}$ es $\Delta_{+}$. Supongamos que

$$
S_{\text {borde }} \ni \int_{z=\epsilon} d^{d} x \sqrt{g_{\epsilon}} \phi(x, \epsilon) \mathcal{O}(x, \epsilon)=\int d^{d} x\left(\frac{R}{\epsilon}\right)^{d} \epsilon^{\Delta_{-}} \phi_{-}^{r e n}(x) \mathcal{O}(x, \epsilon)
$$

en donde $g_{\epsilon}$ es la métrica de AdS evaluada en $z=\epsilon$. Pidiendo que esta expresión sea finita cuando $\epsilon \rightarrow 0$ se tiene

$$
\mathcal{O}(x, \epsilon) \sim \epsilon^{d-\Delta_{-}} \mathcal{O}^{r e n}(x)=\epsilon^{\Delta_{+}} \mathcal{O}^{r e n}(x)
$$

y por lo tanto la dimensión de escala de $\mathcal{O}^{r e n}$ es $\Delta_{+}$.

- Si $m^{2}>0$ entonces $\Delta_{+}>d$ y el operador $\mathcal{O}$ es irrelevante. Esto significa que si perturbamos la CFT con este operador su coeficiente tendrá una escala de masa negativa y por lo tanto sus efectos no serán importantes en el IR ( $z$ grande).

- El operador $\mathcal{O}$ es marginal si $m^{2}=0$.

- Si $m^{2}<0$ entonces $\Delta_{+}<d$ y el operador $\mathcal{O}$ es relevante en el IR. Hay que destacar que en AdS, masas negativas están permitidas siempre y cuando se satisfaga la cota de BreitenlohnerFreedman [18] $m^{2}>-\left|m_{B F}\right|^{2}=-\left(\frac{d}{2 R}\right)^{2}$. Valores de $m^{2}$ dentro de esta cota aseguran energía positiva y por lo tanto una teoría bien definida. 
Volvamos a la ecuación diferencial (1.14). Para valores de $k^{\mu}$ tipo espacio $\left(k^{2}>0\right)$ la solución es

$$
f_{k}(z)=A(k) z^{d / 2} K_{\nu}(k z)+B(k) z^{d / 2} I_{\nu}(k z)
$$

en donde $A(k), B(k)$ son constantes arbitrarias y $K_{\nu}, I_{\nu}$ son funciones de Bessel. Incidentalmente el caso $k^{2}>0$ coincide con la ecuación diferencial que se obtiene en el caso de signatura Euclídea. Regularidad en el interior de AdS $(z=\infty)$ requiere $B(k)=0$ y la solución de (1.14) es entonces única si fijamos los coeficientes $A(k)$. Dichos coeficientes $A(k)=\phi_{+}(k)$ se relacionan con la transformada de Fourier de la condición de contorno $\phi_{+}(x)$ en $z=\epsilon$ de la siguiente manera: normalizando la función de onda para que $f_{k}(z=\epsilon)=1$

$$
f_{k}(z)=\frac{z^{d / 2} K_{\nu}(k z)}{\epsilon^{d / 2} K_{\nu}(k \epsilon)} .
$$

La solución de (1.14) con condición de contorno (1.18) es

$$
\phi(x, z)=\int d^{d} k e^{i k x} f_{k}(z) \phi(k, \epsilon),
$$

con $\phi(k, \epsilon)=\int d x e^{-i k x} \phi(x, \epsilon)$. La acción on-shell resulta

$$
S(\phi)=2 R^{d-1} \int d^{d} k \phi(k, \epsilon) \phi(-k, \epsilon) \epsilon^{-d+1} \partial_{z}\left(\frac{z^{d / 2} K_{\nu}(k z)}{\epsilon^{d / 2} K_{\nu}(k \epsilon)}\right)_{z=\epsilon} .
$$

Expandiendo en serie la función de Bessel vemos que tenemos dos tipos de contribuciones a la acción. Por un lado se obtienen términos con potencias enteras de $k$, es decir analíticos en $k$, estos son términos de contacto y pueden ser substraídos tomando contratérminos en la acción (1.11). La contribución física, no analítica en $k$ tiene la forma,

$$
-\frac{(-1)^{\nu-1} 2^{1-2 \nu}}{\Gamma(\nu)^{2}} k^{2 \nu} \ln k \epsilon . \epsilon^{2 \nu-d}+\ldots
$$

La transformada de Fourier de este término y la apropiada condición de borde (1.18) permiten obtener la función de dos puntos (finita) para el operador $\mathcal{O}$, el resultado es

$$
\left\langle\mathcal{O}\left(x_{1}\right) \mathcal{O}\left(x_{2}\right)\right\rangle=-\frac{\delta}{\delta \phi_{-}^{r e n}\left(x_{1}\right)} \frac{\delta}{\delta \phi_{-}^{r e n}\left(x_{2}\right)} S=\frac{2 \Gamma\left(\Delta_{+}\right)}{\pi^{d / 2} \Gamma\left(\Delta_{+}-d / 2\right)} \frac{1}{\left(x_{1}-x_{2}\right)^{2 \Delta_{+}}} .
$$

En el caso de $k^{\mu}$ tipo tiempo, es decir $k^{2}<0$, las dos soluciones linealmente independientes tienen un comportamiento asintótico similar en $z \rightarrow \infty$

$$
z^{d / 2} K_{ \pm \nu}(i q z) \sim e^{ \pm-i q z}
$$

En este caso regularidad en el interior no elimina una de las soluciones. Esta ambigüedad tiene su origen en que existen diferentes elecciones para la función de Green en $\mathrm{AdS}_{d+1}$. Por ejemplo, una posible elección es la función de Green retardada, la cual da cuenta de la respuesta causal del sistema ante una perturbación. 
En el capítulo 2 se extenderá en detalle la discusión hecha aquí arriba para $\operatorname{AdS}_{d+1}$ en coordenadas globales y signatura Lorentziana. Además se utilizará la prescripción GKPW para calcular funciones de dos puntos en espacios con dos bordes. En particular se estudiará la función de correlación en un agujero de gusano que resuelve las ecuaciones de movimiento de una acción de Einstein-Gauss-Bonnet (EGB) cuyos bordes son asintóticamente AdS. Además se revisará la prescripción de Gubser-Klebanov-Polyakov-Witten (GKPW) [4, 5] para extraer correladores de la QFT a partir de cálculos de gravedad y se discutirá su aplicación a la solución de agujero de gusano Lorentziana encontrada en [37]. A lo largo del camino se mencionarán similitudes y diferencias con el caso de $\mathrm{AdS}_{2}$ (ver $[30,39]$ para otros estudios sobre el agujero de gusano analizado en esta tesis). Recordemos que la prescripción GKPW en signatura Lorentziana involucra no solo conocer datos en el borde del espacio-tiempo sino también el conocimiento de los estados inicial y final, veremos cómo estos estados aparecen en el cálculo (ver [40, 14, 15, 16, 41, 42] para discusiones sobre temas relacionados a la prescripción GKPW en signatura Lorentziana).

\subsubsection{Lazos de Wilson}

El confinamiento de quarks en teorías de gauge no abelianas aún no se ha demostrado debido al hecho de que este fenómeno ocurre en el regimen de acoplamiento fuerte, lo cual nos impide tratarlo con técnicas perturbativas de teoría cuántica de campos. Sin embargo, Wilson propuso [43] un criterio para establecer cuándo una teoría es confinante y cuándo no lo es. Dicho criterio establece que el confinamiento del flujo cromoeléctrico (cuerda de QCD) ocurre en teorías para las cuales el valor de expectación del lazo de Wilson es proporcional al área encerrada por él. En particular, esta ley de área para un contorno rectangular en el espacio-tiempo (banda infinita) correspondiente a un par quark-antiquark estático, corresponde a un potencial confinante lineal en la distancia de separación entre quarks.

En esta sección vamos a estudiar cómo la conjetura AdS/CFT permite estudiar valores de expectación de operadores no locales como el lazo de Wilson y cómo el resultado obtenido estudiando teoría de cuerdas concuerda con los resultados esperados para la teoría de campos.

\section{Teoría de Gauge}

Esencialmente un lazo de Wilson es un factor de fase en teorías de gauge tanto Abelianas como no Abelianas. Estos operadores son invariantes de gauge y por lo tanto son observables de la teoría cuántica. En QCD permite medir el potencial de interacción entre un par de quarks.

La linea de Wilson se define como

$$
U_{\mathcal{C}}(y, z)=\mathcal{P} e^{i \int_{\mathcal{C}} d x^{\mu} A_{\mu}^{a} T^{a}}
$$


donde $\mathcal{C}$ es una curva que conecta $(y, z)$. El operador $U_{\mathcal{C}}(y, z)$ nos provee del valor de un campo $\varphi(y)$ transportando paralelamente el valor de $\varphi(z)$ a lo largo de la curva $\mathcal{C}$ como

$$
\varphi_{\mathcal{C}}(y)=U_{\mathcal{C}}(y, z) \varphi(z) .
$$

$U_{\mathcal{C}}(y, z)$ satisface $D_{\mu} U_{\mathcal{C}}(y, z)=0$ donde $D_{\mu}=\partial_{\mu}+i A_{\mu}$ es la derivada covariante. El símbolo $\mathcal{P}$ en (1.28) nos indica que debemos tomar el orden de caminos, es decir, como en una teoría no Abeliana el campo de gauge $A_{\mu}(x)$ no conmuta en puntos diferentes debemos ordenar el camino de tal forma que en la expansión en serie de la exponencial (1.28) campos evaluados en valores mayores del parámetro $t$ que describe la curva $\mathcal{C}$ queden a la izquierda. $T^{a}$ son los generadores del grupo $G$ en alguna de sus representaciones [44]. El lazo de Wilson se define como la traza de una linea de Wilson a lo largo de una curva cerrada

$$
W_{\mathcal{C}}(\mathcal{R})=\operatorname{Tr} \mathcal{P} e^{i \oint_{\mathcal{C}} d x^{\mu} A_{\mu}^{a} T_{\mathcal{R}}^{a}}
$$

El operador así definido depende solamente de la representación $\mathcal{R}$ del grupo de gauge y del camino $\mathcal{C}$. Este operador invariante de gauge sirve para expandir cualquier función de $A_{\mu}$ invariante de gauge. También se utiliza para distinguir entre las fases de confinamiento y deconfinamiento de una teoría de gauge. Veamos este última propiedad con más detalle: Físicamente el lazo de Wilson describe la contribución del campo de Yang-Mills a la amplitud de propagación de una partícula muy masiva, externa y cargada ante la representación $\mathcal{R}$ del grupo que se mueve a lo largo de $\mathcal{C}$. Si $\mathcal{C}$ es un camino rectangular que tiene una longitud $T$ a lo largo del eje temporal y una separación espacial $l(T \gg l)$, su valor de expectación se comporta como

$$
\left\langle W_{\mathcal{C}}(\square)\right\rangle \sim e^{-T V(l)},
$$

en donde $V$ es el potencial quark-antiquark estático y $\square$ denota la representación fundamental de $S U(N)$. La teoría es linealmente confinante si $V(l) \sim l$. Nótese que para un potencial confinante lineal el argumento de la exponencial en (1.31) es el área encerrada por la curva que define $\mathcal{C}$. Esta es la denominada ley de área. Por otro lado, para QED con quarks externos infinitamente masivos se encuentra que $V(l) \sim \frac{1}{l}$, es decir es de tipo Coulomb, el comportamiento esperado para una teoría conforme.

\section{Teoría de Cuerdas}

Veremos ahora cuál es la prescripción en el lado de cuerdas de la conjetura que nos permite calcular el valor de expectación del lazo de Wilson. En particular voy a enfocarme con detalle en el caso en que el camino $\mathcal{C}$ es un rectángulo y la representación $\mathcal{R}$ es la fundamental. Más adelante en esta sección comentaré cómo obtener el valor de expectación en otras representaciones del grupo de gauge. 


\section{- Representación Fundamental}

En su construcción la conjetura goza de una simetría de gauge $U(N)$ cuyo origen son las cuerdas cuyos extremos terminan en cualquiera de las $N$ D3-branas que se apilan paralelamente unas a otras y no guardan distancia perpendicular entre ellas. Las cuerdas cuyos extremos se ubican sobre diferentes branas son no masivas debido a que no hay una separación física entre las mismas. Consideremos $N+1$ D3-branas ubicadas en el mismo punto del espacio, generando un grupo de gauge $U(N+1)$. Tomemos una de ellas y separémosla del resto, la cuantización del sistema muestra que este procedimiento es la realización del mecanismo de Higgs donde se rompe la simetría de gauge $U(N+1) \rightarrow U(N) \times U(1)$. La cuerda que tiene un extremo sobre cualquiera de las $N$ branas y el otro extremo en la brana alejada será masiva y su masa será proporcional a la distancia de separación $\rho$ entre las branas, asimismo transformará en la representación fundamental del grupo $U(N)$. El extremo de la cuerda es fuente para campos de gauge $U(N)$, y debe ser interpretado como un quark en la representación fundamental. Obtendremos un quark (externo) infinitamente masivo tomando $\rho \rightarrow \infty$. Si tenemos en cuenta la backreaction de las $N D 3$-branas, la cuerda se extenderá a lo largo de la dirección radial de AdS y su hoja de mundo formará una superficie suave.

Como el dual gravitatorio de $\mathcal{N}=4$ es $\mathrm{AdS}_{5} \times S^{5}$, debemos también especificar cómo se extiende la cuerda en la 5 -esfera parametrizada por los ángulos $\theta^{I}$. Debido a esto, se encuentra que la hoja de mundo descripta anteriormente no es fuente para el lazo de Wilson usual sino que lo es para uno supersimétrico, acoplándose también a los campos escalares $X^{I}, I=1, \ldots, 6$ de $\mathcal{N}=4 \mathrm{SYM}$

$$
W_{\mathcal{C}, \mathcal{C}_{\text {int }}}(\square)=\operatorname{Tr} \mathcal{P} e^{\oint\left(i A_{\mu} \dot{x}^{\mu}+\theta^{I} X^{I}\left(x^{\mu}\right) \sqrt{\dot{x}^{2}}\right) d \tau}
$$

En esta expresión $x^{\mu}(\tau)$ parametriza el camino $\mathcal{C}, \theta^{I}(\tau)$ denota el acoplo a los escalares $X^{I}$. $\mathcal{C}_{\text {int }}$ denota la curva sobre el espacio interno. En esta tesis voy a considerar el caso $\theta^{I}=$ constante, es decir, la cuerda se sitúa en un punto sobre la $S^{5}$.

La prescripción para obtener el valor de expectación del lazo de Wilson [45, 46], dice que debemos calcular la función de partición de la cuerda cuya hoja de mundo termina sobre la curva $\mathcal{C}$

$$
\left\langle W_{\mathcal{C}, \mathcal{C}_{\text {int }}}(\square)\right\rangle=\mathcal{Z}_{\text {string }}\left[\mathcal{C}, \mathcal{C}_{\text {int }}, \square\right]
$$

En el límite de supergravedad $g_{s} \rightarrow 0, \lambda \rightarrow \infty$ (y fijo) se tiene

$$
\mathcal{Z}_{\text {string }}\left[\mathcal{C}, \mathcal{C}_{\text {int }}, \square\right] \cong e^{-S_{\text {string }}^{\text {ons shell }}\left[\mathcal{C}, \mathcal{C}_{\text {int }}\right]},
$$

en donde $S_{\text {string }}$ es la acción de Nambu-Goto evaluada en la solución clásica para la cuerda cuyos extremos se ubican sobre la curva $\mathcal{C}$. La mayor contribución a la integral funcional se obtendrá para la/las configuraciones de área mínima. Antes de pasar a calcular el potencial quark-antiquark 
explícitamente, debo hacer notar que dicha área mínima es divergente debido a que el volumen de mundo de la cuerda se extiende hasta el borde de AdS. Esta divergencia es interpretada de la siguiente manera: lo que en realidad estamos calculando es la energía del par quark-antiquark incluyendo la auto-energía debida a su masa infinita. Dicha masa debe ser substraída, la misma es equivalente a la hoja de mundo recta que se extiende desde el origen de AdS hasta infinito. En resumen, el valor de expectación del lazo de Wilson se calcula a partir del área mínima de la hoja de mundo que termina sobre el camino $\mathcal{C}$ en el borde (ver figura 1.3) convenientemente renormalizada.

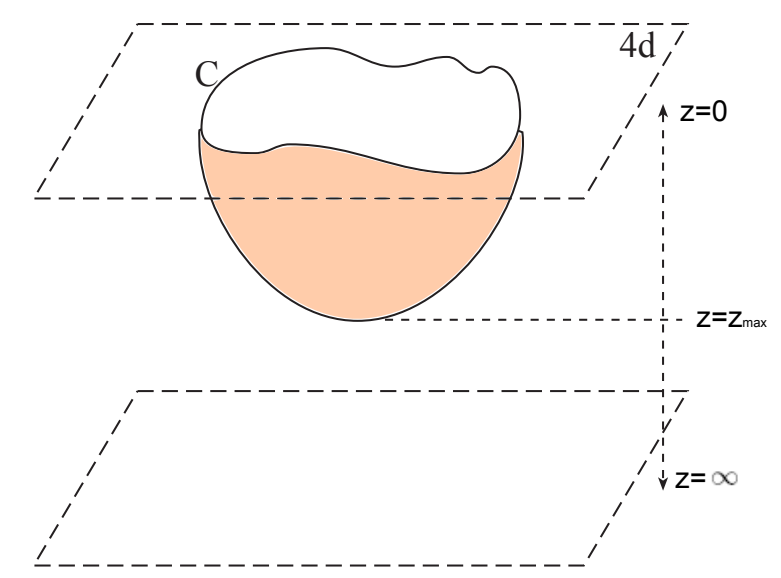

Figura 1.3: Hoja de mundo de la cuerda que tiene a $\mathcal{C}$ como borde. La teoría de gauge vive en el borde del espacio $(z=0)$.

\section{Potencial quark-antiquark}

Estudiemos ahora el potencial quark-antiquark en $\mathcal{N}=4$ a partir de la correspondencia AdS/CFT calculando el valor de expectación del lazo de Wilson por medio de la ecuación (1.34) $[45,46]$. Para hallar dicho potencial la curva $\mathcal{C}$ es un rectángulo de lados $l, T$ con $T \gg l$ (ver figura 1.4). Primero debemos parametrizar la hoja de mundo de la cuerda $X^{\mu}(\tau, \sigma)$ con $, \mu=0, \ldots, 9$ y decidir cómo está inmersa en AdS. La inmersión es la siguiente

$$
\tau=t, \quad \sigma=x, \quad z=z(x)
$$

Las condiciones de contorno para nuestra configuración son $\tau \in\left(-\frac{T}{2}, \frac{T}{2}\right), \sigma \in\left(-\frac{l}{2}, \frac{l}{2}\right)$ y $z\left( \pm \frac{l}{2}\right)=0$, $l$ debe ser interpretado como la distancia de separación entre quarks en la teoría de gauge. 


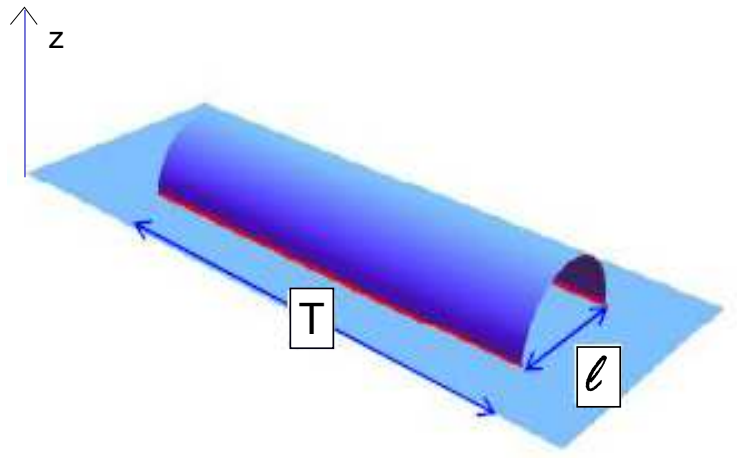

Figura 1.4: Hoja de mundo de la cuerda que tiene al rectángulo de lados $T$ y $l$ como borde. La teoría de gauge vive en el borde del espacio $(z=0)$.

La métrica inducida sobre la hoja de mundo $g_{\alpha \beta}=\partial_{\alpha} X^{\mu} \partial_{\beta} X^{\nu} G_{\mu \nu}(X)$ para el embedding (1.35) queda

$$
d s^{2}=\frac{R^{2}}{z^{2}}\left[-d t^{2}+d x^{2}\left(1+z^{\prime 2}\right)\right]=\gamma_{a b} d x^{a} d x^{b}
$$

en donde $z^{\prime}=\frac{d z}{d x}$. La acción de Nambu-Goto para la cuerda se escribe

$$
\begin{aligned}
S_{N G} & =\frac{1}{2 \pi \alpha^{\prime}} \int_{-T / 2}^{T / 2} d t \int_{-l / 2}^{l / 2} d x \sqrt{-\operatorname{det}(\gamma)} \\
& =\sqrt{4 \pi \lambda} T \int_{-l / 2}^{l / 2} d x \frac{\sqrt{1+z^{\prime 2}}}{z^{2}} .
\end{aligned}
$$

Encontrar la superficie mínima en este caso es análogo a un problema de mecánica clásica en una variable. El Lagrangiano tiene una simetría traslacional en $x$ y por lo tanto existe una corriente conservada $\mathcal{H}$ asociada a la misma dada por

$$
\begin{aligned}
\mathcal{H} & =\mathcal{L}-z^{\prime} \frac{\partial \mathcal{L}}{\partial z^{\prime}} \\
& =\frac{1}{z^{2} \sqrt{1+z^{\prime 2}}}=\frac{1}{z_{\text {max }}^{2}}
\end{aligned}
$$

en donde $\mathcal{L}$ es el Lagrangiano de Nambu-Goto y $z_{\max }$ el máximo valor alcanzado por la hoja de mundo en el interior de AdS (ver figura 1.5). Esta ecuación puede reescribirse como un problema unidimensional en un potencial efectivo $V_{e f}(z)$ ( $z_{\max }$ es el punto de retorno)

$$
z^{\prime 2}+V_{e f}(z)=0, \quad V_{e f}(z)=1-\left(\frac{z_{\max }}{z}\right)^{4}
$$

A partir de (1.38) podemos despejar $x^{\prime}(z)$ y calcular la separación entre quarks $l$ como función de $z_{\max }$ obteniendo

$$
l=\int_{-l / 2}^{l / 2} d x=2 \int_{0}^{z_{\max }} d z \frac{d x}{d z}=2 z_{\max } \int_{0}^{1} d y \frac{y^{2}}{\sqrt{1-y^{4}}}=2 z_{\max } \frac{\sqrt{2 \pi^{3}}}{\Gamma^{2}(1 / 4)},
$$




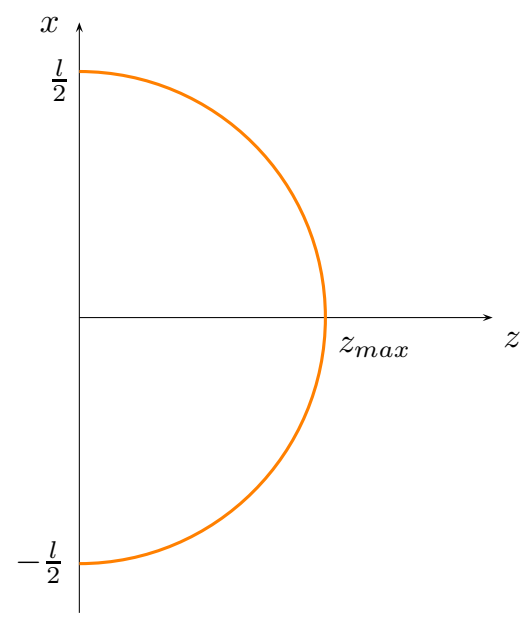

Figura 1.5: Plano $x-z$. La curva naranja muestra el perfil $z(x)$ de la cuerda en AdS, alcanzando un valor máximo $z_{\max }$.

Nótese que al aumentar la separación $l$ entre quarks en el borde, la cuerda explora regiones más interiores del espacio AdS. Sustituyendo la expresión para $z^{\prime}$ obtenida de (1.39) en la acción de Nambu-Goto queda

$$
S_{N G}=2 \sqrt{4 \pi \lambda} T \int_{\epsilon}^{z_{\max }} d z \frac{z_{\max }^{2}}{z^{2} \sqrt{z_{\max }^{4}-z^{4}}} .
$$

La divergencia para $z \rightarrow 0$ en esta expresión se debe como mencionamos anteriormente a que la hoja de mundo se extiende hasta el borde de AdS. Para regularizarla, sustraemos la auto-energía de los quarks restando el área de dos superficies planas que terminan sobre dos líneas rectas y se extienden a lo largo de todo el espacio AdS

$$
m_{q}=\sqrt{4 \pi \lambda} T \int_{\epsilon}^{z_{\max }} \frac{d z}{z^{2}}
$$

Luego

$$
\ln \left\langle W_{\mathcal{C}}(\square)\right\rangle=S_{N G}-2 m_{q}=-\frac{2 \sqrt{4 \pi \lambda} T}{z_{\max }} \int_{\epsilon / z_{\max }}^{1} \frac{d y}{y^{2}}\left(\frac{1}{\sqrt{1-y^{4}}}-1\right),
$$

y el potencial quark-antiquark queda

$$
V(l)=-\frac{\sqrt{\lambda}}{l} \frac{4 \pi^{2}}{\Gamma^{4}(1 / 4)} .
$$

Nótese que la energía se comporta como $1 / l$ y es proporcional a $\sqrt{\lambda}$. Lo primero era esperable ya que $\mathcal{N}=4$ es invariante de escala. Con respecto al coeficiente de proporcionalidad se debe decir que está predicción de AdS/CFT sugiere un apantallamiento en el régimen de acoplamiento fuerte. 


\section{Lazo de Wilson circular}

Para estudiar el lazo de Wilson definido sobre una curva circular spacelike [47, 48] es conveniente escribir la métrica de AdS (1.10) (en signatura Euclídea) en la forma

$$
d s^{2}=\frac{1}{z^{2}}\left(d r^{2}+r^{2} d \phi^{2}+d z^{2}+d x_{i}^{2}\right) .
$$

En este caso la solución para la hoja de mundo de la cuerda (ver figura 1.6) es

$$
z=\sqrt{a^{2}-r^{2}}, \quad 0 \leq r \leq a, \quad 0 \leq \phi \leq 2 \pi
$$

en donde $a$ denota el radio del círculo en el borde de AdS. Calculando el área mínima de la superficie que termina sobre el cárculo (propiamente regularizada) se encuentra que

$$
\ln \left\langle W_{\mathcal{C}}(\square)\right\rangle=-\sqrt{\lambda}
$$

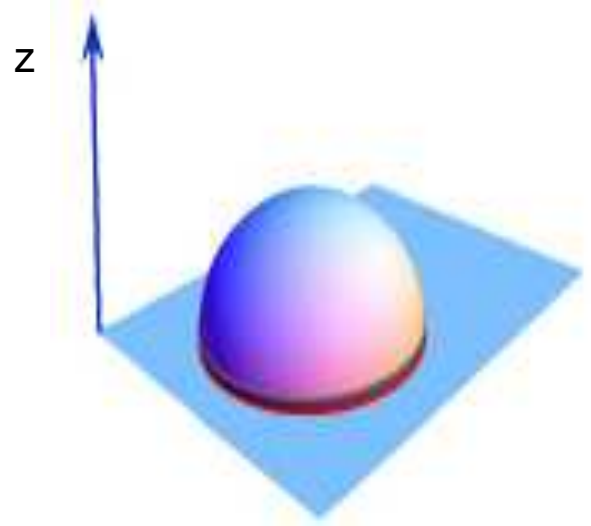

Figura 1.6: Hoja de mundo de la cuerda que tiene al circulo de radio a como borde.

Tanto para la línea de Wilson como para el lazo circular se demostró en $[49,50]$ que el valor de expectación obtenido mediante AdS/CFT coincide exactamente con lo obtenido en $\mathcal{N}=4$ SYM a todo orden en teoría de perturbaciones.

\section{- Representaciones simétrica y antisimétrica, líneas y círculos}

La descripción gravitatoria dual para líneas de Wilson supersimétrico en $\mathcal{N}=4$ en la representación simétrica o antisimétrica del grupo fue propuesta en [51] y [52] respectivamente. En [51] se argumento que soluciones de $D 3$-branas enroscadas de forma tal que su volumen de mundo sea $\mathrm{AdS}_{2} \times S^{2} \subset \mathrm{AdS}_{5}$, con campo eléctrico encendido y ubicadas en un punto de $S^{5}$ son duales al valor de expectación del lazo de Wilson en la representación simétrica. El flujo del campo eléctrico caracteriza el número de bloques de la representación. Por otro lado, la representación antisimétrica 
se obtiene enroscando $D 5$ branas [52] (también eléctricamente cargadas) con el objetivo de obtener un volumen de mundo $\mathrm{AdS}_{2} \times S^{4}$ donde $\mathrm{AdS}_{2} \subset \mathrm{AdS}_{5}$ y $S^{4} \subset S^{5}$. En ambos casos, la guía para obtener estas a priori caprichosas formas de embeber las branas en $\operatorname{AdS}_{5} \times S^{5}$ fue la simetría. El lazo de Wilson 1/2-BPS descripto en la ecuación (1.32) para una curva tipo tiempo $\mathcal{C}$ recta o un circulo espacial es invariante ante el grupo $O s p\left(4^{*} \mid 4\right) \approx S U(1,1) \times S U(2) \times S O(5)$ [94]. La conjetura nos dice que debemos realizar estas simetrías en el lado de gravedad y la forma natural de hacerlo es enroscando las $D 3$ o $D 5$ branas como ha sido explicado mas arriba. La presencia de un campo eléctrico da cuenta de la carga $[53,54]$. Al igual que en la representación fundamental, en el límite semiclásico debemos minimizar la acción de Dirac-Born-Infeld (DBI) para la D-brana y calcular su valor evaluándola en la solución de las ecuaciones de movimiento para el embedding. En la representación simétrica el resultado obtenido en el caso en que $\mathcal{C}$ es un circulo tipo espacio es [51]

$$
\ln \left\langle W_{\mathcal{C}}(S)\right\rangle=2 N\left(\kappa \sqrt{1+\kappa^{2}}+\sinh ^{-1} \kappa\right),
$$

en donde $\kappa$ es la constante de proporcionalidad del campo eléctrico. En la representación antisimétrica se obtiene [52]

$$
\ln \left\langle W_{\mathcal{C}}(A)\right\rangle=-\frac{2 N \sqrt{\lambda}}{3 \pi} \sin ^{3} \theta_{k},
$$

siendo $\theta_{k}$ la posición de la D5-brana en la $S^{5}$. El subíndice $k$ da cuenta de que dicha posición depende del campo eléctrico y determina el orden de la representación antisimétrica (número de bloques en el diagrama de Young).

En [55] se confirmaron estos resultados desde el punto de vista de la teoría conforme $\mathcal{N}=4$ utilizando técnicas de localización y modelo de matrices.

\subsection{Aplicaciones a materia condensada}

En los último años la conjetura de Maldacena ha sido utilizada para modelar la física de materia condensada. En particular la idea es usar la correspondencia AdS/CFT para estudiar materiales no convencionales que desafían los paradigmas tradicionales de la física de materia condensada como, por ejemplo, materiales que involucran electrones fuertemente correlacionados. Esta aplicación de la correspondencia ha sido llamada AdS/CMT [71].

La técnica convencional para estudiar sistemas de materia condensada se basa en dos pilares fundamentales: la teoría del líquido de Fermi, la cual estudia propiedades de electrones en sólidos como pequeñas perturbaciones de estado fundamental, y la teoría de Landau, que afirma que al producirse una transición de fase debe haber una ruptura de simetría.

Recientemente diferentes experimentos con materiales no convencionales mostraron que en 
muchas situaciones de interés la teoría de Fermi no es una descripción adecuada del problema. Una famoso indicativo de esto fue que la resistividad eléctrica observada era proporcional a la temperatura $T$ y no a $T^{2}$ como predice la teoría de Fermi.

Lo que sucede en realidad es que las hipótesis de estas teorías no son validas, esto sucede por ejemplo en sistemas fuertemente correlacionados tales como superconductores con alta temperatura crítica, compuestos de fermiones pesados, líquidos con efecto Hall cuántico fraccionario en gases de electrones 2-dimensionales y líquidos de Lüttinger en una dimensión. En estos sistemas ocurren transiciones de fase que no se relacionan con la ruptura de ninguna simetría y asimismo presentan propiedades que no pueden ser descriptas con la teoría de perturbaciones usual de un líquido de Fermi.

$\mathrm{Al}$ estar en un regimen de acoplamiento fuerte la correspondencia AdS/CFT postula un dual gravitatorio para dichos sistemas. La idea en esta extensión de la conjetura es dar cuenta cualitativamente de fenómenos que no cuentan con una descripción adecuada. La aplicación de la conjetura AdS/CFT a estos sistemas persigue el objetivo de dar un respuesta desde el punto de vista gravitatorio a la siguiente pregunta que nos podemos hacer desde el punto de vista de la teoría de campos: ¿Cómo podemos clasificar todas las posibles fases gapless de la materia a baja temperatura? Las teorías de campos libres exhiben dos fases a bajas temperatura. Un bosón cargado libre será sometido a una condensación de Bose-Einstein, rompiendo espontáneamente la simetría de carga. El grado de libertad gapless es en consecuencia un bosón de Goldstone. Por otro lado, los fermiones libres cargados pueden formar una superficie de Fermi y los grados de libertad gapless son las excitaciones de dicha superficie. La dinámica de los bosones de Goldstone y de las excitaciones de la superficie de Fermi está estrechamente condicionada por la cinemática y está bien entendida. Para teorías fuertemente interactuantes la pregunta sobre las posibles fases de la materia se torna más complicada de responder, aquí es en donde la perspectiva gravitacional es útil.

En general, los fondos gravitatorios duales a un sistema de materia condensada involucran geometrías de agujeros negros cargados, la dualidad prescribe que su horizonte y su carga eléctrica son mapeados a la temperatura y el potencial químico de la teoría de campos. Las técnicas desarrolladas permiten estudiar, por ejemplo, conductividad Hall [56], efecto Nernst [57] y la ley de Ohm [58] en un régimen de acoplamiento fuerte del sistema de materia condensada.

La mayoría de los modelos AdS/CMT son del tipo bottom-up, es decir, son efectivos y en general no se derivan de la teoría de cuerdas. El objetivo de estos modelos es dar una descripción macroscópica/cualitativa de ciertos fenómenos que ocurren en sistemas de materia condensada.

Hay muchos trabajos con aplicaciones de la conjetura al estudio de sistemas superfluídos y superconductores $[40,59,60,61,62]$, líquidos de Fermi (FL) [63, 64], líquidos que no cumplen con 
la teoría de Fermi (NFL) [65, 66, 67], líquidos cuánticos semi-locales [68], puntos críticos cuánticos y aislantes topológicos $[69,70]$ entre otras interesantes teorías de materia condensada.

En el capítulo 4 se estudiará en detalle cómo funciona AdS/CMT aplicada al estudio de un superconductor tipo $p$. 
Los resultados obtenidos en el desarrollo de esta tesis fueron presentados en las siguientes reuniones científicas

- XXXVIII reunión de la red Strings@ar, IAFE, Buenos Aires. Septiembre 2009.

- XLV reunión de la red Strings@ar, IAFE, Buenos Aires. Marzo 2011.

- Encuentro de Becarios del IFLP, La Plata. Diciembre 2010 y Diciembre 2012.

- International School on String Theory and Fundamental Physics, DESY, Hamburg, Germany. Julio 2012.

y han sido documentados en las siguientes publicaciones científicas internacionales con referato:

- Wilson loop stability in the gauge/gravity correspondence.

Raúl Arias y Guillermo Silva.

JHEP 1001 (2010) 023;

arXiv:0911.0662 [hep-th]

- Lorentzian AdS, Wormholes and Holography.

Raúl Arias, Marcelo Botta Cantcheff y Guillermo Silva.

Phys.Rev. D83 (2011) 066015;

arXiv:1012.4478 [hep-th]

- Backreacting $p$-wave superconductors

Raúl Arias e Ignacio Salazar Landea.

JHEP 1301 (2013) 157;

arXiv:1210.6823 [hep-th] 


\section{Capítulo 2}

\section{AdS Lorentziano, Agujeros de Gusano y Holografía}

En este capítulo se investigará la estructura de funciones de dos puntos para la QFT dual a un agujero de gusano Lorentziano. La geometría en el volumen es una solución 5-dimensional de la gravedad de Einstein-Gauss-Bonnet y conecta causalmente dos espacio-tiempos asintóticamente AdS. Se resumirá la prescripción de GKPW para calcular funciones de correlación de dos puntos para los operadores duales $\mathcal{O}$ en signatura Lorentziana y se propondrá expresar los campos en el

volumen en términos de su valor en los bordes asintóticamente AdS independientes $\phi_{0}^{ \pm}$, a lo largo del camino se mostrará cómo la ambigüedad de agregar modos normalizables en el volumen se relaciona con los estados inicial y final sobre los que se calcula el correlador. Los valores en el borde son interpretados como fuentes para los operadores duales $\mathcal{O}^{ \pm}$y se argumentará que, además de la posibilidad de entrelazamiento, existe un acoplamiento entre los grados de libertad definidos en cada uno de los bordes. Se discutirá también la geometría $\mathrm{AdS}_{1+1}$ debido a sus estructuras de borde similar. Basado en el análisis, se propondrá un criterio geométrico que permitirá distinguir efectos de entrelazamiento y efectos de acoplamiento entre los dos conjuntos de grados de libertad asociados a cada uno de los bordes desconectados.

Las geometrías asintóticamente AdS cumplen un rol destacado en la correspondencia AdS/CFT $[1,4,5]$ ya que estas proveen duales gravitatorios para teorías cuánticas de campos (QFT) con puntos fijos conformes en el UV. Existe un consenso general, basado en un gran número de comprobaciones, para la interpretación dual de diferentes soluciones asintóticamente AdS: una solución de agujero negro se supone describe una QFT térmica [19], una solución que interpola entre un horizonte de AdS (correspondiente a un punto fijo IR) y una geometría de AdS en infinito con diferente radio realiza el flujo del grupo de renormalización entre dos puntos conformes [20]. Una tercera posibilidad son ciertos solitones (cargados) en AdS que se interpretan como estados coherentes excitados de la QFT [21]. 
Se discutirá la problemática que se genera cuando la solución de gravedad es un agujero de gusano que conecta causalmente dos bordes asintóticamente $\mathrm{AdS}_{d+1}$ (Lorentzianos). La holografía y la correspondencia AdS/CFT en presencia de múltiples bordes aún no están bien comprendidas. La implementación del paradigma AdS/CFT en tales casos sugiere que la teoría de campos dual vive en la unión de dos bordes disjuntos, y entonces es el producto de las teorías de campos de los diferentes bordes (ver [22]). Voy a rever esta afirmación y discutir cuándo las dos teorías duales son independientes, están acopladas o son desacopladas.

Para signatura Lorentziana, las geometrías de agujero de gusano están descartadas (en $d \geq 2$ dimensiones) como soluciones de la acción de Einstein-Hilbert satisfaciendo condiciones naturales de causalidad: bordes desconectados deben estar separados por horizontes [23] ([24] es un trabajo reciente en $2+1$ dimensiones). Por lo tanto, los estudios de agujeros de gusano en teoría de cuerdas y en el contexto de la correspondencia AdS/CFT se han concentrado en espacios de signatura Euclídea, particularmente motivados por sus aplicaciones a cosmología (ver referencias de [25, 26]). En el contexto Euclídeo existe un teorema que establece que los espacios con bordes desconectados (de curvatura escalar positiva) también están descartados como soluciones de Einstein sobre variedades de curvatura negativa [27] (ver también [28]). Además, [27] prueba que para bordes de curvatura negativa la teoría de campos dual definida sobre ellos debe ser inestable para $d \geq 3$ (ver también [29]). Los agujeros de gusano estudiados en [25] evitan el teorema de [27] debido a que fueron construidos como rebanadas hiperbólicas de AdS soportados con campos de supergravedad extras.

El ejemplo canónico de un espacio con dos bordes, con signatura Lorentziana, separados por un horizonte es el agujero negro eterno. En [31] se hace contacto entre esta geometría y la formulación dinámica de campos térmicos (TFD) de una QFT a temperatura finita [34]: los dos bordes desconectados dan lugar a dos copias desacopladas $\mathcal{H}_{ \pm}$de la teoría de campos dual y los correladores no nulos $\left\langle\mathcal{O}^{+}(\mathbf{x}) \mathcal{O}^{-}\left(\mathbf{x}^{\prime}\right)\right\rangle$ se interpretan como promedios sobre un estado entrelazado que codifica la información estadística/térmica de la geometría (ver también [35] y [32]). Un segundo ejemplo Lorentziano de interés con dos bordes desconectados fue construido en [33] trabajando con un orbifolio no singular de $\mathrm{AdS}_{3}$. El resultado de la construcción condujo a dos bordes cilíndricos causalmente conectados y su teoría de campos dual involucra el límite DLCQ de la teoría conforme que vive en una configuración de D1-D5 branas, pero el acoplamiento entre los grados de libertad de los diferentes bordes no ha sido clarificado. La diferencia principal entre estos dos ejemplos es que en el último caso existe contacto causal entre los bordes conformes. El teorema [23] se elude en el segundo caso debido a que el orbifold tiene una dirección compacta ( $S^{1}$ en este caso) fibrada sobre $\mathrm{AdS}_{2}$, en donde el mencionado teorema no aplica.

Los teoremas [23] que prohíben agujeros de gusano Lorentzianos no son válidos cuando se 
trabaja con gravedades de orden más alto. Por otro lado, correcciones en la curvatura de orden más alto a la gravedad estándar de Einstein son esperables en una teoría cuántica de la gravedad. Sin embargo, no se conoce mucho acerca de la forma precisa de las correcciones en derivadas de orden superior más que en unos pocos casos máximamente supersimétricos. Como desde el punto de vista puramente gravitatorio la teoría más general que involucra ecuaciones de campo de segundo orden para la métrica es del tipo de Lovelock [36], voy a elegir trabajar con la más simple de ellas conocida como teoría de Einstein-Gauss-Bonnet. La acción para esta teoría solo contiene términos hasta orden cuadrático en la curvatura y mi interés en la solución de agujero de gusano, encontrada en [37], es que permite un tratamiento analítico. La geometría corresponde a un agujero de gusano estático que conecta dos regiones localmente asintóticas AdS con variedad

de base $\tilde{\Sigma}$, la cual en $d+1=5$ toma la forma $\tilde{\Sigma}=H^{3}$ o $S^{1} \times H^{2}$, en donde $H^{2}$ y $H^{3}$ son espacios hiperbólicos (cocientados) dos y tres- dimensionales. La geometría resultante es suave, no contiene horizontes, y las dos regiones asintóticas están causalmente conectadas. En el caso de la solución en 5 dimensiones [37] se realizó un análisis de estabilidad en [30].

Es comúnmente aceptado que la QFT dual a una geometría de agujero de gusano debe corresponder a dos teorías de gauge independientes definidas sobre cada uno de los bordes y la geometría del agujero de gusano codifica la información de un estado entrelazado entre ellos. Por otro lado, la conexión causal entre los bordes da lugar a un acoplamiento no trivial entre las dos teorías duales [33]. Se va a argumentar, continuando analíticamente a una sección Euclídea del espacio-tiempo, que el resultado no nulo obtenido para el correlador $\left\langle\mathcal{O}^{+}(\mathbf{x}) \mathcal{O}^{-}\left(\mathbf{x}^{\prime}\right)\right\rangle$ entre operadores localizados en bordes opuestos señala la existencia de un acoplamiento entre los campos asociados a cada uno de los bordes.

El capítulo se organizará de la siguiente manera: en la sección 2.1 se revisará la prescripción GKPW para calcular correladores de la QFT a partir de la teoría de gravedad mencionando las peculiaridades de la signatura Lorentziana. En la sección 2.2 se extenderá la prescripción GKPW para el caso de datos sobre dos bordes asintóticos independientes. Se aplicará esto a $\mathrm{AdS}_{2}$, reproduciendo los resultados que aparecen en la literatura, y al agujero de gusano [37] mostrando sus similitudes. En la sección 2.3 se discutirán varios argumentos relacionados con la posibilidad de entrelazamiento y/o interacción entre las dos QFT duales.

\subsection{Prescripción GKPW con un solo borde asintótico}

En esta sección se reformulará lo estudiado en la sección 1.1.2 en términos de funciones de Green, con el objeto de facilitar la extensión de la prescripción GKPW a espacios con más de un borde que se realizará en la siguiente sección. 
La prescripción de GKPW [4, 5] iguala la función de partición de gravedad para un espaciotiempo $\mathcal{M}$ asintóticamente $\mathrm{AdS}_{d+1}$, entendida como una funcional de los datos de borde, a la funcional generatriz de funciones de correlación para una teoría de campos conforme (CFT) definida sobre el borde conforme del espacio-tiempo $\partial \mathcal{M}$. Explícitamente la prescripción es

$$
\mathcal{Z}_{\text {gravity }}\left[\phi\left(\phi_{0}\right)\right]=\left\langle e^{i \int_{\partial \mathcal{M}} d^{d} \mathbf{x} \phi_{0}(\mathbf{x}) \mathcal{O}(\mathbf{x})}\right\rangle
$$

En el lado izquierdo $\phi_{0}=\phi_{0}(\mathbf{x})$ es el valor en el borde del campo $\phi$, el lado derecho es la funcional generatriz de correladores en la CFT para operadores $\mathcal{O}$ duales al campo $\phi$. En este capítulo se trabajará en el límite semiclásico del espacio-tiempo (límite de $N$ grande en la CFT) y por lo tanto el lado izquierdo en (2.1) podrá ser aproximado por la acción on-shell del campo $\phi$, el cual por simplicidad será tomado como un campo escalar de masa $m$. Se centrará el interés en geometrías de tiempo real (Lorentzianas) debido a que la prescripción (2.1) en este caso se encuentra incompleta, esto se debe a la necesidad de especificar los estados inicial y final $\psi_{\mathrm{i}, \mathrm{f}}$ sobre los cuales se calcula los correladores del lado derecho.

Para fijar la notación se resumirá la prescripción para campos escalares masivos resaltando los puntos importantes para nuestros argumentos. La métrica de $A d S_{d+1}$ en coordenadas de Poincaré es

$$
d s^{2}=\frac{R^{2}}{z^{2}}\left(d \mathbf{x}^{2}+d z^{2}\right),
$$

en donde el termino $d \mathbf{x}^{2}$ significa $-d t^{2}+d \vec{x}^{2}$. El borde (conforme) de AdS se ubica en $z=0 \mathrm{y}$ existe un horizonte en $z=\infty^{1}$. La solución para la ecuación de movimiento para el campo $\phi$ sujeto a datos de borde $\phi_{0}$ se escribe comúnmente

$$
\phi(\mathbf{x}, z)=\int_{\partial \mathcal{M}} d \mathbf{y K}(\mathbf{x}, z \mid \mathbf{y}) \phi_{0}(\mathbf{y}) .
$$

En el límite de campo libre, la ecuación de Klein-Gordon (KG) muestra que el comportamiento asintótico para $\phi$ es

$$
\phi(\mathbf{x}, z) \sim z^{\Delta_{ \pm}} \phi_{0}(\mathbf{x}), \quad z \rightarrow 0
$$

en donde ${ }^{2}$

$$
\Delta_{ \pm}=\frac{d}{2} \pm \mu, \quad \mu=\sqrt{\frac{d^{2}}{4}+m^{2} R^{2}} .
$$

El propagador volumen-borde $\mathrm{K}$ en $(2.3)$ debe satisfacer $[4,5]$

$$
\left(\square-m^{2}\right) \mathrm{K}(\mathbf{x}, z \mid \mathbf{y})=0
$$

\footnotetext{
${ }^{1}$ En el caso Euclídeo $z=\infty$ es solo un punto, lo que conduce a un semiplano $z \geq 0$ en (2.2) compactificado a una esfera.

${ }^{2}$ Campos escalares de masa negativa están permitidos en AdS siempre que $\mu \geq 0$. El mínimo permitido para la masa del campo escalar en $\operatorname{AdS}_{d+1}$ esta dado por la llamada cota de Breitenlohner-Freedman (BF) $\mu_{B F}=0$, o equivalentemente $m_{B F}^{2}=-d^{2} / 4[18]$.
} 
con condiciones de borde

$$
\mathrm{K}(\mathbf{x}, z \mid \mathbf{y}) \sim z^{\Delta_{-}} \delta(\mathbf{x}-\mathbf{y}), \quad z \rightarrow 0
$$

Finalmente, el propagador volumen-borde K puede ser relacionado con la función de Green (Dirichlet) volumen-volumen $\mathrm{G}\left(\mathbf{x}, z \mid \mathbf{y}, z^{\prime}\right)$ a través de la segunda identidad de Green, resulta entonces que $\mathrm{K}$ puede ser obtenido como la derivada normal de $\mathrm{G}$ evaluada en el borde del espacio-tiempo (ver $[72,73])$ como

$$
\mathrm{K}(\mathbf{x}, z \mid \mathbf{y})=\lim _{z^{\prime} \rightarrow 0} \sqrt{-g} g^{z^{\prime} z^{\prime}} \partial_{z^{\prime}} \mathrm{G}\left(\mathbf{x}, z \mid \mathbf{y}, z^{\prime}\right) .
$$

Dos comentarios vienen al caso: i) la solución en el volumen del espacio para unas dadas condiciones de borde $\phi_{0}$ calculadas a partir de (2.3) no es única ya que espacios AdS Lorentzianos admiten soluciones normalizables $\varphi(\mathbf{x}, z)$ que pueden sumarse a (2.3) sin alterar el comportamiento en el borde (2.7), explícitamente

$$
\phi(\mathbf{x}, z)=\int_{\partial \mathcal{M}} d \mathbf{y K}(\mathbf{x}, z \mid \mathbf{y}) \phi_{0}(\mathbf{y})+\varphi(\mathbf{x}, z) .
$$

La consecuencia de su inclusión en la CFT se interpreta como que se están fijando los estados inicial y final $\left|\psi_{\mathrm{i}, \mathrm{f}}\right\rangle$ sobre los cuales se calculan los valores de expectación en el lado derecho de (2.1). La segunda observación es ii) en signatura Lorentziana la superficie $z=\infty$ es un horizonte de Killing y por lo tanto se necesita especificar el campo sobre un borde adicional para obtener un problema de Dirichlet bien definido (ver fig.2.2(a)). Estas dos observaciones están relacionadas al echo de se requiere una segunda condición para fijar completamente el propagador volumen-borde $\mathrm{K}$ (recordar que en espacio Euclídeo pedir regularidad en el volumen implica $K \rightarrow 0$ cuando $z \rightarrow \infty$ ). La condición faltante sobre $\mathrm{K}$ impuesta en el horizonte $(z=\infty)$ se expresa mejor en términos de modos de Fourier de ondas puramente entrantes (funciones que decaen exponencialmente) para momento tipo tiempo (espacio), este es un problema bien conocido de las teorías cuánticas de campos en espacio-tiempos curvos y se reduce a la elección de un vacío. La incorporación de modos normalizables (tipo tiempo) induce una componente saliente del horizonte, la cual se interpreta naturalmente como una excitación (ver $[4,14,15,16]$ y $[32,74,75,76]$ para trabajos relacionados).

Me interesa calcular la función de correlación de dos puntos en la teoría de campos dual. Con este fin se necesita la acción evaluada en la solución para un campo escalar a orden cuadrático

$$
S=-\frac{1}{2} \int d \mathbf{x} d z \sqrt{-g}\left(g^{\mu \nu} \partial_{\mu} \phi \partial_{\nu} \phi+m^{2} \phi^{2}\right) .
$$

integrando por partes y evaluando en la solución, la contribución del borde conforme esta dado por (ver [75] para una discusión sobre posibles contribuciones del horizonte)

$$
S\left[\phi_{0}\right]=\frac{1}{2} \int d \mathbf{x}\left[\sqrt{-g} g^{z z} \phi(\mathbf{x}, z) \partial_{z} \phi(\mathbf{x}, z)\right]_{z=0} .
$$


Insertando (2.3) en esta expresión obtenemos la acción evaluada en la solución como una funcional del valor del campo en el borde $\phi_{0}$

$$
S\left[\phi_{0}\right]=\frac{1}{2} \int d \mathbf{y} d \mathbf{y}^{\prime} \phi_{0}(\mathbf{y}) \Delta\left(\mathbf{y}, \mathbf{y}^{\prime}\right) \phi_{0}\left(\mathbf{y}^{\prime}\right)
$$

en donde

$$
\Delta\left(\mathbf{y}, \mathbf{y}^{\prime}\right)=\int d \mathbf{x}\left[\sqrt{-g} g^{z z} \mathrm{~K}(\mathbf{x}, z \mid \mathbf{y}) \partial_{z} \mathrm{~K}\left(\mathbf{x}, z \mid \mathbf{y}^{\prime}\right)\right]_{z=0} .
$$

Teniendo en cuenta (2.7) y (2.8) en (2.13) se obtiene

$$
\begin{aligned}
\Delta\left(\mathbf{y}, \mathbf{y}^{\prime}\right) & \sim\left[\sqrt{-g} g^{z z} \partial_{z} \mathrm{~K}\left(\mathbf{y}, z \mid \mathbf{y}^{\prime}\right)\right]_{z=0} \\
& \sim \lim _{z, z^{\prime} \rightarrow 0}\left(\sqrt{-g} g^{z z}\right)\left(\sqrt{-g} g^{z^{\prime} z^{\prime}}\right) \frac{\partial^{2}}{\partial z \partial z^{\prime}} \mathrm{G}\left(\mathbf{y}, z \mid \mathbf{y}^{\prime}, z^{\prime}\right) .
\end{aligned}
$$

Esta relación ha sido usada para relacionar, en el límite semiclásico, la función de dos puntos con las geodésicas de la geometría [77].

Resumiendo, la función de dos puntos para un operador $\mathcal{O}$ dual al campo $\phi$ se obtiene a partir de la acción evaluada en la solución como

$$
\left\langle\psi_{\mathrm{f}}\left|\mathcal{O}(\mathbf{y}) \mathcal{O}\left(\mathbf{y}^{\prime}\right)\right| \psi_{\mathrm{i}}\right\rangle=-i \frac{\delta^{2} S\left[\phi_{0}\right]}{\delta \phi_{0}(\mathbf{y}) \delta \phi_{0}\left(\mathbf{y}^{\prime}\right)}=-i \Delta^{\mathrm{i}, \mathrm{f}}\left(\mathbf{y}, \mathbf{y}^{\prime}\right) .
$$

Los estados inicial y final $\psi_{\mathrm{i}, \mathrm{f}}$ del lado izquierdo involucran la ambigüedad ante la adición de una solución normalizable a (2.3), en la siguiente sección se verá explícitamente como se manifiestan estos estados en (2.13).

\section{AdS en coordenadas globales}

La receta para obtener correladores de una teoría de campos a partir de cálculos de gravedad involucra evaluar cantidades, definidas en el volumen del espacio-tiempo, en el borde conforme, como puede sospecharse de (2.4), (2.7) y (2.13) la evaluación conduce a singularidades y por lo tanto se necesita una regularización. En lo que sigue se discutirá cómo se hace ésto en AdS expresado en coordenadas globales debido a que el caso del agujero de gusano discutido después coincide en su región asintótica con este sistema de coordenadas y será por lo tanto regularizado de la misma manera. En el camino se mostrará cómo aparece en los cálculos la especificación de los estados inicial y final $\psi_{\mathrm{i}, \mathrm{f}}$.

La regularización de (2.16) se realiza imponiendo la condición de borde en alguna distancia finita en el volumen y tomando el límite al borde al final de los cálculos (ver [78] para una sutileza al tomar este límite). La variedad $\mathrm{AdS}_{d+1}$ esta totalmente cubierta por las llamadas coordenadas globales, en las cuales la métrica toma la forma

$$
d s^{2}=R^{2}\left[-\frac{d t^{2}}{1-x^{2}}+\frac{d x^{2}}{\left(1-x^{2}\right)^{2}}+\frac{x^{2}}{1-x^{2}} d \Omega_{d-1}^{2}\right]
$$


en donde se ha cambiado la variable $\rho$ a $x=\tanh \rho$ para mapear el borde conforme a $x=1$.

Se imponen los datos en el borde a una distancia finita $x_{\epsilon}=1-\epsilon$, luego, por consistencia se necesita que

$$
\lim _{x \rightarrow x_{\epsilon}} \mathrm{K}\left(t, \Omega, x \mid t^{\prime}, \Omega^{\prime}, x_{\epsilon}\right)=\frac{\delta\left(t-t^{\prime}\right) \delta\left(\Omega-\Omega^{\prime}\right)}{\sqrt{g_{\Omega}}} .
$$

y $\mathrm{K}$ regular en el interior. El propagador volumen-borde $\mathrm{K}$ que satisface (2.18) puede ser obtenido a partir de la solución de la ecuaciones de Klein-Gordon $\phi(t, \Omega, x)=e^{-i \omega t} Y_{l m}(\Omega) f_{l \omega}(x)$ como

$$
\mathrm{K}\left(t, \Omega, x \mid t^{\prime}, \Omega^{\prime}, x_{\epsilon}\right)=\int_{-\infty}^{\infty} \frac{d \omega}{2 \pi} \sum_{l m} e^{-i \omega\left(t-t^{\prime}\right)} Y_{l m}(\Omega) Y_{l m}^{*}\left(\Omega^{\prime}\right) f_{l \omega}(x)
$$

si se normaliza ${ }^{3} f_{l \omega}\left(x_{\epsilon}\right)=1$. La ecuación diferencial que satisface $f_{l \omega}(x)$ es

$$
\left(1-x^{2}\right) \frac{d^{2} f_{l \omega}}{d x^{2}}+\frac{d-1-x^{2}}{x} \frac{d f_{l \omega}}{d x}+\left(\omega^{2}-\frac{q^{2}}{x^{2}}-\frac{m^{2} R^{2}}{1-x^{2}}\right) f_{l \omega}=0 .
$$

La solución de esta ecuación es una combinación lineal de dos funciones hipergeométricas, pero una de ellas diverge cuando $x \rightarrow 0$ y entonces pedir regularidad en el volumen del espacio demanda anularla. La solución regular propiamente normalizada es

$f_{l \omega}(x)=\left(\frac{x^{-\frac{d}{2}+\nu+1}\left(1-x^{2}\right)^{\frac{1}{2} \Delta_{+}}}{(1-\epsilon)^{-\frac{d}{2}+\nu+1}((2-\epsilon) \epsilon)^{\frac{1}{2} \Delta_{+}}}\right) \frac{{ }_{2} F_{1}\left(\frac{1}{2}(\mu+\nu-\omega+1), \frac{1}{2}(\mu+\nu+\omega+1) ; \nu+1 ; x^{2}\right)}{{ }_{2} F_{1}\left(\frac{1}{2}(\mu+\nu-\omega+1), \frac{1}{2}(\mu+\nu+\omega+1) ; \nu+1 ;(1-\epsilon)^{2}\right)}$

aquí ${ }_{2} F_{1}$ es la función hipergeométrica de Gauss con $\mu$ dado por $(2.5)$ y $\nu=\sqrt{\left(\frac{d-2}{2}\right)^{2}+q^{2}}$, la simetría de la función hipergeométrica en sus primeros dos argumentos implica que $f_{l \omega}(x)=$ $f_{l-\omega}(x)$. El comportamiento asintótico de la solución (2.21) cerca del borde va como

$$
f_{l \omega}(x) \sim C_{+}(1-x)^{\frac{1}{2} \Delta_{+}}+C_{-}(1-x)^{\frac{1}{2} \Delta_{-}},
$$

en donde $\Delta_{ \pm}$esta dado por $(2.5)$ y $C_{ \pm}=C_{ \pm}(\mu, \nu, \omega)$. En signatura Lorentziana el operador de $\mathrm{KG}$ posee soluciones normalizables, estas aparecen para valores particulares de $\omega$ dados por $[18,14,79]$

$$
\begin{aligned}
\omega_{n l} & = \pm(2 n+\nu+\mu+1) \\
& = \pm\left(2 n+l+\Delta_{+}\right), \quad n, l=0,1,2 \ldots
\end{aligned}
$$

o dicho de otra manera, estas son las frecuencias para las cuales ${ }^{4} C_{-}=0$. La discretitud del espectro manifiesta el carácter tipo " caja"de AdS y desde la perspectiva dual proviene del echo de que $S^{3}$ es una geometría compacta. La cuantización de los estados (2.23) en el volumen se interpreta como dual a los estados definidos sobre el borde conforme $S^{3} \times \mathbb{R}$ de AdS.

\footnotetext{
${ }^{3}$ Los esféricos armónicos $Y_{l m}$ sobre la esfera $d-1$ satisfacen $\nabla^{2} Y_{l m}=-q^{2} Y_{l m}$ con $q^{2}=l(l+d-2), l=0,1, \ldots$

${ }^{4}$ ver [18] para una condición de cuantización alternativa para $0 \leq \mu \leq 1$ y [80] para su interpretación en el contexto de AdS/CFT.
} 


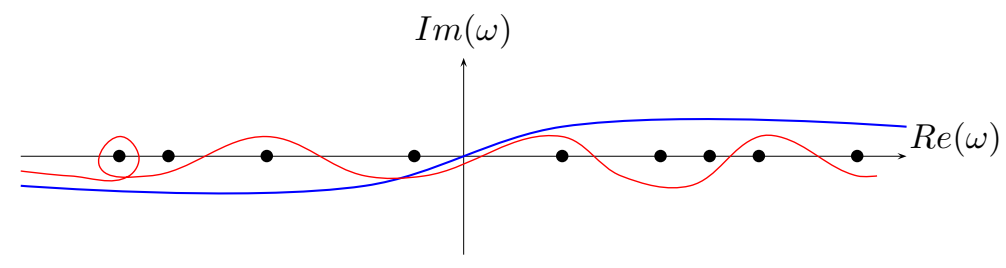

Figura 2.1: Contornos en el plano complejo $\omega$ : al hacer la integración sobre $\omega$ en $(2.27)$ cualquier contorno arbitrario (dibujado en rojo) puede ser deformado al contorno de Feynman (dibujado en azul) más contribuciones provenientes de rodear los polos (2.23). El rodeo de polos positivos (negativos) fijan los estados inicial (final) $\psi_{\mathrm{i}, \mathrm{f}}$ en (2.16).

La función de dos puntos para los operadores de la QFT dual se obtienen de insertar

$$
\phi(t, \Omega, x)=\int d t^{\prime} d \Omega^{\prime} \sqrt{g_{\Omega^{\prime}}} \mathrm{K}\left(t, \Omega, x \mid t^{\prime}, \Omega^{\prime}, x_{\epsilon}\right) \phi_{0}\left(t^{\prime}, \Omega^{\prime}\right)
$$

en la acción (2.10), nótese que no se incluyeron soluciones normalizables a (2.24) (ver próximo párrafo). La acción evaluada en la solución conduce a un termino de borde evaluado en $x_{\epsilon}$ (ver (2.11)-(2.13)) y la expresión regularizada para $\Delta\left(t, \Omega \mid t^{\prime}, \Omega^{\prime}\right)$ en $(2.12)$ se escribe como ${ }^{5}$

$$
\begin{aligned}
\Delta_{\mathrm{reg}}\left(t, \Omega \mid t^{\prime}, \Omega^{\prime}\right) & =-\frac{1}{\sqrt{g_{\Omega}}}\left[\sqrt{-g} g^{x x} \partial_{x} \mathrm{~K}\left(t, \Omega, x \mid t^{\prime}, \Omega^{\prime}, x_{\epsilon}\right)\right]_{x=x_{\epsilon}} \\
& =-\int \frac{d \omega}{2 \pi} e^{-i \omega\left(t-t^{\prime}\right)} \sum_{l m} Y_{l m}(\Omega) Y_{l m}^{*}\left(\Omega^{\prime}\right)\left[\frac{x^{d-1}}{\left(1-x^{2}\right)^{\frac{d-2}{2}}} \partial_{x} f_{l \omega}(x)\right]_{x=x_{\epsilon}} \\
& =-\sum_{l m} Y_{l m}(\Omega) Y_{l m}^{*}\left(\Omega^{\prime}\right) \int \frac{d \omega}{2 \pi} e^{-i \omega\left(t-t^{\prime}\right)}\left[\frac{x^{d-1}}{\left(1-x^{2}\right)^{\frac{d-2}{2}}} \partial_{x} f_{l \omega}(x)\right]_{x=x_{\epsilon}}
\end{aligned}
$$

en donde la primera linea proviene de (2.13) teniendo en cuenta (2.18).

Algunos comentarios sobre (2.25): cuando se toma el límite $\epsilon \rightarrow 0$, la expresión en la última linea es ambigua debido a la existencia de polos simples ubicados a lo largo del contorno de integración en el plano $\omega$ en $(2.23){ }^{6}$. Estos polos manifiestan la existencia de soluciones normalizables en el volumen de la geometría (ver (2.9)) y entonces, para definir el contorno de integración en $\omega$ se necesita alguna prescripción para evitar los polos. La elección del contorno se entiende tradicionalmente como la elección entre las funciones de Green avanzada/retardada/Feynman, en lo que sigue se trabajará con la de Feynman. Quiero llamar la atención acerca de la observación (ver [42]) sobre la relación entre contornos en el plano complejo $\omega$ y la elección de soluciones normalizables. La observación es simple, cualquier elección particular de contornos es equivalente por deformación a elegir el contorno de Feynman más contribuciones que provendrán de rodear los polos (2.23). Luego, la ambigüedad en la expresión (2.24) proveniente de la adición de modos normalizables

\footnotetext{
${ }^{5}$ Siguiendo [78], cuando se calcula (2.25) primero se calcula la derivada en $x$ y después se toma el límite $\epsilon \rightarrow 0$. ${ }^{6} \mathrm{Su}$ origen se debe a haber pedido $f_{l \omega}\left(x_{\epsilon}\right)=1$.
} 
arbitrarios se translada en una elección del contorno en el plano complejo $\omega$ (ver fig. 2.1). El contorno de Feynman naturalmente conduce a correladores ordenados temporalmente, y el rodeo de modos normalizables positivos (negativos) fija el estado inicial (final) $\psi_{\mathrm{i}, \mathrm{f}}$ en el lado izquierdo de (2.16). La elección del contorno retardado da lugar a funciones respuesta en lugar de funciones de correlación. En resumen, los estados son interpretados como creados a partir de un fundamental $\left|\psi_{0}\right\rangle$ asociado al contorno de integración de referencia elegido.

El límite $\epsilon \rightarrow 0$ de la expresión dentro de los corchetes en (2.25) también presenta polos (analíticos y no analíticos) en $\epsilon$. El resultado físico se obtiene renormalizando los datos en el borde teniendo en cuenta el comportamiento asintótico en la dirección radial (ver (2.22)), en el presente caso esto lleva a reescalear $\phi_{0}$ como (ver $[5,78,73]$ )

$$
\phi_{0}(t, \Omega)=\epsilon^{\frac{1}{2} \Delta_{-}} \phi_{\mathrm{ren}}(t, \Omega)
$$

Además, como eventualmente estamos interesados en funciones de correlación para puntos separados, no se deben tener en cuenta términos de contacto proporcionales a potencias enteras positivas de $q^{2}$. El término finito en el límite $\epsilon \rightarrow 0$ es

$$
\begin{aligned}
\Delta_{\mathrm{ren}}\left(t, \Omega \mid t^{\prime}, \Omega^{\prime}\right) \equiv & \lim _{\epsilon \rightarrow 0} \epsilon^{\Delta_{-}} \Delta_{\mathrm{reg}}\left(t, \Omega \mid t^{\prime}, \Omega^{\prime}\right) \\
= & \sum_{l m} Y_{l m}(\Omega) Y_{l m}^{*}\left(\Omega^{\prime}\right) \int \frac{d \omega}{2 \pi} e^{-i \omega\left(t-t^{\prime}\right)} \\
& \times \frac{\Delta_{+}}{2^{\Delta_{-}}} \frac{\Gamma(1-\mu)}{\Gamma(1+\mu)} \frac{\Gamma\left(\frac{1}{2}(-\omega+\nu+\mu+1)\right) \Gamma\left(\frac{1}{2}(\omega+\nu+\mu+1)\right)}{\Gamma\left(\frac{1}{2}(-\omega+\nu-\mu+1)\right) \Gamma\left(\frac{1}{2}(\omega+\nu-\mu+1)\right)} .
\end{aligned}
$$

El numerador de esta última expresión muestra explícitamente la aparición de polos a lo largo del contorno de integración precisamente a las frecuencias $\omega_{n l}$ dadas por (2.23). La especificación de un contorno $\mathcal{C}$ en el plano complejo $\omega$ fija los estados inicial y final $\psi_{\mathrm{i}, \mathrm{f}}$ cuando se compara con el camino de Feynman estándar (ver fig. 2.1).

A partir de la discusión que sigue a (2.25) debe quedar claro que las funciones de correlación calculadas en el estado de vacío de la QFT se obtienen de elegir el contorno de Feynman para la integración en $\omega$ en (2.27). Integrando sobre $\omega$ se obtiene ${ }^{7}$

$$
\begin{aligned}
\Delta_{\text {ren }}^{F}\left(t, \Omega \mid t^{\prime}, \Omega^{\prime}\right)= & 2 i \frac{\Delta_{+} \Gamma(1-\mu)}{2^{\Delta_{-}} \Gamma(1+\mu)} \sum_{l m} Y_{l m}(\Omega) Y_{l m}^{*}\left(\Omega^{\prime}\right) \\
& \times\left[\sum_{n=0}^{\infty} \frac{(-1)^{n}}{n !} \frac{\Gamma\left(n+l+\Delta_{+}\right)}{\Gamma\left(n+l+\frac{d}{2}\right) \Gamma(-(n+\mu))} e^{-i\left|t-t^{\prime}\right|\left(2 n+l+\Delta_{+}\right)}\right]
\end{aligned}
$$

\footnotetext{
${ }^{7}$ Generalmente (2.23) son las únicas divergencias en (2.27), se debe tener un cuidado especial para valores enteros de $\mu$. No se discutirán los detalles en esta tesis debido a que la experiencia con la correspondencia AdS/CFT ha mostrado que la función de correlación no cambia cualitativamente en este límite.
} 


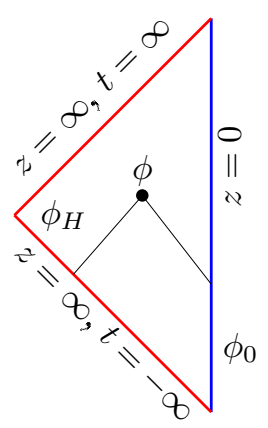

(a)

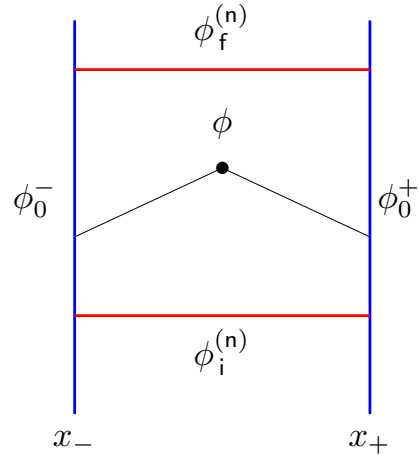

(b)

Figura 2.2: (a) Coordenadas de Poincare para AdS Lorentziano: el valor del campo escalar $\phi$ en cualquier punto del volumen no solo depende del valor en el borde $\phi_{0}$ sino que también depende del valor del campo en los horizontes pasado y futuro $\phi_{H}$. $\quad$ (b) Geometría de Agujero de Gusano Lorentziano: el valor del campo escalar en el volumen no solo depende de los dos valores de borde $\phi_{0}^{ \pm}$(azul) sino que también depende de los modos normalizables en el volumen $\phi^{(\mathrm{n})}$ (rojo). La dependencia, en ambos gráficos, sobre los modos normalizables (líneas rojas) se correlata con la elección de los estados inicial y final $\left|\psi_{\mathrm{i}, \mathrm{f}}\right\rangle$ y se manifiesta como la elección de un contorno de integración en el plano complejo $\omega$ cuando se calculan los correladores (2.27).

La suma sobre los residuos conduce a

$$
\begin{aligned}
\left\langle 0\left|T \mathcal{O}(t, \Omega) \mathcal{O}\left(t^{\prime}, \Omega^{\prime}\right)\right| 0\right\rangle= & -i \Delta_{\text {ren }}^{F}\left(t, \Omega \mid t^{\prime}, \Omega^{\prime}\right)=-\frac{2 \Delta_{+}}{2^{\Delta_{-}} \Gamma(\mu)} \sum_{l m} Y_{l m}(\Omega) Y_{l m}^{*}\left(\Omega^{\prime}\right) \frac{\Gamma\left(l+\Delta_{+}\right)}{\Gamma\left(l+\frac{d}{2}\right)} \\
& \times e^{-i\left|t-t^{\prime}\right|\left(l+\Delta_{+}\right)}{ }_{2} F_{1}\left(1+\mu, l+\Delta_{+}, l+\frac{d}{2} ; e^{-2 i\left|t-t^{\prime}\right|}\right)
\end{aligned}
$$

\subsection{Prescripción GKPW para agujeros de gusano Lorentzianos}

El gol en esta sección será extender la prescripción de GKPW al caso de múltiples bordes tipo tiempo, por simplicidad se discutirá el caso con dos bordes. Sobre fondos gravitatorios generales la correspondencia AdS/CFT sugiere que la presencia de dos bordes tipo tiempo se debe asociar con la existencia de dos conjuntos de operadores duales $\mathcal{O}^{ \pm}$correspondientes a las dos condiciones de borde independientes $\phi_{0}^{ \pm}$que deben ser impuestas al campo $\phi$ cuando se resuelve la ecuación de onda ${ }^{8}$.

Se considerarán agujeros de gusano con topología en el borde conforme de la forma $\mathbb{R} \times \Sigma$, con $\mathbb{R}$ representando el tiempo y $\Sigma=\Sigma_{+}+\Sigma_{-}$la unión de las dos copias (espaciales) compactas y disjuntas $\Sigma_{ \pm}$. Los agujeros de gusano pueden cubrirse por un sistema de coordenadas $(x, t, \theta)$ en donde $x$ es la coordenada holográfica radial en el volumen y $\left(x_{ \pm}, t, \theta\right)$ las coordenadas que parametrizan los dos bordes $\mathbb{R} \times \Sigma_{ \pm}$. En presencia de dos bordes conformes desconectados se propone escribir al campo en el volumen en términos de los datos $\phi_{0}^{ \pm}(\mathbf{y})$ sobre cada uno de los

\footnotetext{
${ }^{8}$ Algunos autores asumen que las dos teorías de campos duales están desacopladas debido a la estructura disconexa del borde [35].
} 
bordes como (ver figura 2.2)

$$
\begin{aligned}
\phi(\mathbf{y}, x) & =\int d \mathbf{y}^{\prime} \mathrm{K}^{i}\left(\mathbf{y}, x \mid \mathbf{y}^{\prime}\right) \phi_{0}^{i}\left(\mathbf{y}^{\prime}\right) \\
& =\int d \mathbf{y}^{\prime}\left[\mathrm{K}^{+}\left(\mathbf{y}, x \mid \mathbf{y}^{\prime}\right) \phi_{0}^{+}\left(\mathbf{y}^{\prime}\right)+\mathrm{K}^{-}\left(\mathbf{y}, x \mid \mathbf{y}^{\prime}\right) \phi_{0}^{-}\left(\mathbf{y}^{\prime}\right)\right] .
\end{aligned}
$$

aquí $\mathbf{y}=(t, \theta)$, y nótese que la solución para dadas condiciones de borde no es única debido a que en signatura Lorentziana pueden agregarse soluciones normalizables a (2.30). Esta ambigüedad se resuelve, como se discutió en la sección 2.1, cuando se elige un contorno en el espacio de frecuencias $\omega$ del núcleo K. Mi método difiere de la propuesta desarrollada en [33]: en aquel trabajo solo se discutió el propagador volumen-borde $K^{+}$y su forma fue determinada pidiendo ausencia de cortes cuando se extiende la coordenada radial a valores complejos. La prescripción condujo a la conclusión de que los $\phi_{0}^{ \pm}$no eran independientes.

Consistencia con (2.30) demanda que los propagadores volumen-borde $\mathrm{K}^{ \pm}\left(\mathbf{y}, x \mid \mathbf{y}^{\prime}\right)$ sean solución de la ecuación de Klein-Gordon (2.6) con las siguientes condiciones de contorno

$$
\begin{array}{lll}
\left.\mathrm{K}^{+}\left(\mathbf{y}, x \mid \mathbf{y}^{\prime}\right)\right|_{x=x_{+}} & =\delta\left(\mathbf{y}-\mathbf{y}^{\prime}\right), & \left.\mathrm{K}^{+}\left(\mathbf{y}, x \mid \mathbf{y}^{\prime}\right)\right|_{x=x_{-}}=0 \\
\left.\mathbf{K}^{-}\left(\mathbf{y}, x \mid \mathbf{y}^{\prime}\right)\right|_{x=x_{-}}=\delta\left(\mathbf{y}-\mathbf{y}^{\prime}\right), & \left.\mathrm{K}^{-}\left(\mathbf{y}, x \mid \mathbf{y}^{\prime}\right)\right|_{x=x_{+}}=0
\end{array}
$$

Estas expresiones determinan completamente los propagadores volumen-borde $\mathrm{K}^{ \pm}$. La acción evaluada en la solución (2.10) resulta en dos términos provenientes de los bordes y toma la forma

$$
S=-\frac{1}{2} \int d \mathbf{y}\left(\left[\sqrt{-g} g^{x x} \phi(\mathbf{y}, x) \partial_{x} \phi(\mathbf{y}, x)\right]_{x=x_{+}}-\left[\sqrt{-g} g^{x x} \phi(\mathbf{y}, x) \partial_{x} \phi(\mathbf{y}, x)\right]_{x=x_{-}}\right)
$$

Insertando la solución (2.30) en (2.32) se obtiene

$$
S\left[\phi_{0}\right]=-\frac{1}{2} \int d \mathbf{y} d \mathbf{y}^{\prime} \phi_{0}^{i}(\mathbf{y}) \Delta_{i j}\left(\mathbf{y}, \mathbf{y}^{\prime}\right) \phi_{0}^{j}\left(\mathbf{y}^{\prime}\right)
$$

con $i, j=+,-$ denotando los dos bordes y $\Delta_{i j}$ la generalización de (2.13). Su forma explícita es

$$
\Delta_{+i}\left(\mathbf{y}, \mathbf{y}^{\prime}\right)=\left[\sqrt{-g} g^{x x} \partial_{x} \mathrm{~K}^{i}\left(\mathbf{y}, x \mid \mathbf{y}^{\prime}\right)\right]_{x=x_{+}}, \quad \Delta_{-i}\left(\mathbf{y}, \mathbf{y}^{\prime}\right)=-\left[\sqrt{-g} g^{x x} \partial_{x} \mathrm{~K}^{i}\left(\mathbf{y}, x \mid \mathbf{y}^{\prime}\right)\right]_{x=x_{-}}
$$

Como en la sección 2.1 la función de dos puntos de operadores en el mismo borde resulta

$$
\left\langle\psi_{\mathrm{f}}\left|\mathcal{O}^{ \pm}(\mathbf{y}) \mathcal{O}^{ \pm}\left(\mathbf{y}^{\prime}\right)\right| \psi_{\mathrm{i}}\right\rangle \sim-i \Delta_{ \pm \pm}\left(\mathbf{y}, \mathbf{y}^{\prime}\right)
$$

y los correladores entre operadores sobre bordes opuestos es

$$
\left\langle\psi_{\mathrm{f}}\left|\mathcal{O}^{ \pm}(\mathbf{y}) \mathcal{O}^{\mp}\left(\mathbf{y}^{\prime}\right)\right| \psi_{\mathrm{i}}\right\rangle \sim-i \Delta_{ \pm \mp}\left(\mathbf{y}, \mathbf{y}^{\prime}\right)
$$

La generalización de las expresiones (2.8) y (2.15) a fondos de gravedad con dos bordes toma la forma

$$
\mathrm{K}^{i}\left(\mathbf{y}, x \mid \mathbf{y}^{\prime}\right)=\lim _{x^{\prime} \rightarrow x^{i}} \sqrt{-g} g^{x^{\prime} x^{\prime}} \partial_{x^{\prime}} \mathrm{G}\left(\mathbf{y}, x \mid \mathbf{y}^{\prime}, x^{\prime}\right)
$$


lo cual da

$$
\Delta_{i j}\left(\mathbf{y}, \mathbf{y}^{\prime}\right) \sim \lim _{x \rightarrow x^{i}, x^{\prime} \rightarrow x^{j}}\left(\sqrt{-g} g^{x x}\right)\left(\sqrt{-g} g^{x^{\prime} x^{\prime}}\right) \frac{\partial^{2}}{\partial_{x} \partial_{x^{\prime}}} \mathrm{G}\left(\mathbf{y}, x \mid \mathbf{y}^{\prime}, x^{\prime}\right) .
$$

Nótese que la función de correlación $\Delta_{ \pm \mp}$ involucra, en el límite semiclásico, una geodésica a través del volumen que conecta los puntos sobre bordes opuestos.

\section{Banda Lorentziana de $\mathrm{AdS}_{2}$}

En esta sección se aplicará la prescripción desarrollada en la subsección anterior para $\mathrm{AdS}_{2}$ Lorentziano, reobteniendo los resultados previos [35]. La métrica Lorentziana de $\mathrm{AdS}_{2}$ puede escribirse como

$$
d s^{2}=R^{2}\left[-\frac{d t^{2}}{1-x^{2}}+\frac{d x^{2}}{\left(1-x^{2}\right)^{2}}\right]
$$

el borde tipo tiempo esta ubicado en $x= \pm 1$ y el sistema de coordenada $(t, x)$ cubre todo el espacio-tiempo. Para encontrar el propagador volumen-borde $\mathrm{K}^{ \pm}$en (2.30) se propone

$$
\mathrm{K}^{ \pm}(t, x)=\int_{-\infty}^{\infty} \frac{d \omega}{2 \pi} e^{-i \omega t} f_{\omega}^{ \pm}(x)
$$

Insertándolo dentro de la ecuación de KG (2.6) se obtiene la ecuación diferencial para $f_{\omega}$

$$
\left(1-x^{2}\right) \frac{d^{2} f_{\omega}^{ \pm}(x)}{d x^{2}}-x \frac{d f_{\omega}^{ \pm}(x)}{d x}+\left(\omega^{2}-\frac{m^{2} R^{2}}{1-x^{2}}\right) f_{\omega}^{ \pm}(x)=0 .
$$

La solución a (2.41) puede escribirse en términos de polinomios generalizados de Legendre como

$$
f_{\omega}^{ \pm}(x)=\left(1-x^{2}\right)^{\frac{1}{4}}\left[a_{\omega}^{ \pm} P_{\nu}^{\mu}(x)+b_{\omega}^{ \pm} Q_{\nu}^{\mu}(x)\right]
$$

con $\mu=\sqrt{\frac{1}{4}+m^{2} R^{2}}, \nu=\omega-\frac{1}{2}$ y $a_{\omega}^{ \pm}, b_{\omega}^{ \pm}$constantes arbitrarias que quedarán fijadas cuando se impongan las condiciones de contorno (2.31). Las condiciones se trasladan en ${ }^{9}$

$$
f_{\omega}^{ \pm}\left( \pm x_{\epsilon}\right)=1, \quad f_{\omega}^{ \pm}\left(\mp x_{\epsilon}\right)=0
$$

Las soluciones a (2.43) son

$$
\begin{gathered}
f_{\omega}^{+}(x)=\left(\frac{1-x^{2}}{1-x_{\epsilon}^{2}}\right)^{\frac{1}{4}} \frac{Q_{\nu}^{\mu}(x) P_{\nu}^{\mu}\left(-x_{\epsilon}\right)-Q_{\nu}^{\mu}\left(-x_{\epsilon}\right) P_{\nu}^{\mu}(x)}{Q_{\nu}^{\mu}\left(x_{\epsilon}\right) P_{\nu}^{\mu}\left(-x_{\epsilon}\right)-Q_{\nu}^{\mu}\left(-x_{\epsilon}\right) P_{\nu}^{\mu}\left(x_{\epsilon}\right)} \\
f_{\omega}^{-}(x)=\left(\frac{1-x^{2}}{1-x_{\epsilon}^{2}}\right)^{\frac{1}{4}} \frac{Q_{\nu}^{\mu}(x) P_{\nu}^{\mu}\left(x_{\epsilon}\right)-Q_{\nu}^{\mu}\left(x_{\epsilon}\right) P_{\nu}^{\mu}(x)}{Q_{\nu}^{\mu}\left(-x_{\epsilon}\right) P_{\nu}^{\mu}\left(x_{\epsilon}\right)-Q_{\nu}^{\mu}\left(x_{\epsilon}\right) P_{\nu}^{\mu}\left(-x_{\epsilon}\right)} .
\end{gathered}
$$

Analizando el comportamiento asintótico cerca del borde en (2.42) se encuentran los modos normalizables para

$$
\omega_{n}= \pm\left(n+\mu+\frac{1}{2}\right), \quad n=0,1,2 \ldots \quad \text { y } \quad \frac{b_{\omega Q}}{a_{\omega Q}}=-\frac{2 \tan \pi \mu}{\pi}
$$

\footnotetext{
${ }^{9}$ Como se discutió en la sección 2.1 el dato en el borde se impone a una distancia finita $x= \pm x_{\epsilon}$ donde $x_{\epsilon}=1-\epsilon$.
} 
Las funciones renormalizadas $\Delta_{i j}$ (sin tener en cuenta términos de contacto) resultan

$$
\begin{aligned}
& \Delta_{\text {ren }_{ \pm \pm}}\left(t, t^{\prime}\right)=\mp \frac{2^{\Delta_{-}}}{2 \pi} \frac{\Gamma(1-\mu)}{\Gamma(1+\mu)} \int \frac{d \omega}{2 \pi} e^{-i \omega\left(t-t^{\prime}\right)} \Gamma\left(\frac{1}{2}+\mu-\omega\right) \Gamma\left(\frac{1}{2}+\mu+\omega\right) \cos (\pi \omega(2.47) \\
& \Delta_{\text {ren } \pm \mp}\left(t, t^{\prime}\right)=\mp \frac{2^{\Delta_{-}}}{\Gamma(\mu)^{2}} \int \frac{d \omega}{2 \pi} e^{-i \omega\left(t-t^{\prime}\right)} \Gamma\left(\frac{1}{2}+\mu-\omega\right) \Gamma\left(\frac{1}{2}+\mu+\omega\right)
\end{aligned}
$$

como antes, los integrandos en estas expresiones muestran polos en $\omega$ para los valores dados por (2.46).

Las integrales (2.47)-(2.48) puede calcularse usando el teorema de los residuos una vez que se haya elegido un contorno en el plano complejo. Para el contorno de Feynman se obtiene

$$
\begin{aligned}
& \Delta_{\text {ren }_{ \pm \pm}}^{F}\left(t, t^{\prime}\right)=\mp\left(\frac{i^{\Delta_{-}-\Delta_{+}}}{8^{\Delta_{+}} \pi^{\frac{1}{2}}}\right) \frac{\Gamma\left(\frac{1}{2}+\mu\right)}{\Gamma(\mu) \sin ^{2 \Delta_{+}}\left(\frac{t-t^{\prime}}{2}\right)} \\
& \Delta_{\text {ren }_{ \pm \mp}}^{F}\left(t, t^{\prime}\right)=\mp\left(\frac{8^{\Delta_{-}} i}{4}\right) \frac{\Gamma(1+2 \mu)}{\Gamma(\mu)^{2} \cos ^{2 \Delta_{+}}\left(\frac{t-t^{\prime}}{2}\right)}
\end{aligned}
$$

El valor de expectación de vacío entre operadores sobre el mismo y opuestos bordes resultan (confrontar con [35])

$$
\begin{aligned}
& \left\langle 0\left|T \mathcal{O}^{ \pm}(t) \mathcal{O}^{ \pm}\left(t^{\prime}\right)\right| 0\right\rangle= \pm\left(\frac{4^{\Delta_{-}} i^{2 \Delta_{-}}}{8^{\Delta_{+}}}\right) \frac{\Gamma(2 \mu)}{\Gamma(\mu)^{2} \sin ^{2 \Delta_{+}}\left(\frac{t-t^{\prime}}{2}\right)}, \\
& \left\langle 0\left|T \mathcal{O}^{ \pm}(t) \mathcal{O}^{\mp}\left(t^{\prime}\right)\right| 0\right\rangle=\mp\left(\frac{8^{\Delta_{-}}}{4}\right) \frac{\Gamma(1+2 \mu)}{\Gamma(\mu)^{2} \cos ^{2 \Delta_{+}\left(\frac{t-t^{\prime}}{2}\right)} .}
\end{aligned}
$$

La primera linea da el resultado para operadores sobre el mismo borde y tiene el comportamiento conforme esperado $\left|t-t^{\prime}\right|^{-2 \Delta_{+}}$cuando los operadores se acercan uno al otro. La segunda linea corresponde a operadores localizados sobre bordes diferentes y son singulares para $t=t^{\prime}+(2 n+1) \pi$, $n \in \mathbb{Z}$, esta singularidad refleja la existencia de curvas causales (nulas) que conectan los dos bordes y se a argumentado que su existencia es una señal de interacción entre los dos conjuntos de grados de libertad $\mathcal{O}^{ \pm}[33]^{10}$. La periodicidad observada en el tiempo se relaciona con una propiedad particular de AdS, esta es la convergencia de geodésicas nulas cuando se pasa al cubrimiento universal y puede ser entendidas como una consecuencia de que los modos propios (2.46) están equiespaciados (ver [79]- [81]).

En el caso sin masa $\left(\mu=\frac{1}{2}\right)$ las funciones de dos puntos toman la forma

$$
\left\langle 0\left|T \mathcal{O}^{ \pm}(t) \mathcal{O}^{ \pm}\left(t^{\prime}\right)\right| 0\right\rangle= \pm \frac{1}{8 \pi \sin ^{2}\left(\frac{t-t^{\prime}}{2}\right)}, \quad\left\langle 0\left|T \mathcal{O}^{ \pm}(t) \mathcal{O}^{\mp}\left(t^{\prime}\right)\right| 0\right\rangle=\mp \frac{1}{4 \pi \cos ^{2}\left(\frac{t-t^{\prime}}{2}\right)}
$$

\section{Agujero de Gusano}

Voy ahora a analizar las funciones de dos puntos en un fondo gravitatorio de agujero de gusano, esto es, una geometría de espacio-tiempo con dos bordes conformes conectados a través del volumen.

\footnotetext{
10 Esta interpretación asume que las foliaciones espaciales del borde $\Sigma_{+}^{(t)}$ y $\Sigma_{-}^{\left(t^{\prime}\right)}$ deben ser identificada como la misma superficie de Cauchy $\tilde{\Sigma}$ de una única variedad de base en donde se define la teoría de campos.
} 
Se trabajará con una agujero de gusano que sirve como modelo de juguete, el cual permite un tratamiento analítico, que consiste de una geometría estática que conecta dos regiones asintóticas localmente AdS con variedad de base de la forma $H^{3}$ o $S^{1} \times H^{2}$, con $H^{n}$ un espacio hiperbólico cocientado $n$ - dimensional. La geometría no contiene horizontes, y las dos regiones asintóticas están causalmente conectadas. El espacio-tiempo fue encontrado como una solución de la gravedad de Einstein-Gauss-Bonnet, la cual en $d+1=5$ dimensiones toma la forma

$$
S_{5}=\kappa \int \epsilon_{a b c d e}\left(R^{a b} R^{c d}+\frac{2}{3 l^{2}} R^{a b} e^{c} e^{d}+\frac{1}{5 l^{4}} e^{a} e^{b} e^{c} e^{d}\right) e^{e},
$$

aquí $R^{a b}=d \omega^{a b}+\omega_{f}^{a} \omega^{f b}$ es la dos forma de curvatura para la conección de spin $\omega^{a b}$, y $e^{a}$ es el vielbein. La métrica $d+1$-dimensional encontrada en [37] es

$$
\begin{aligned}
d s^{2} & =R^{2}\left[-\cosh ^{2} \rho d t^{2}+d \rho^{2}+\cosh ^{2} \rho d \tilde{\Sigma}_{d-1}^{2}\right] \\
& =R^{2}\left[-\frac{d t^{2}}{1-x^{2}}+\frac{d x^{2}}{\left(1-x^{2}\right)^{2}}+\frac{d \tilde{\Sigma}_{d-1}^{2}}{1-x^{2}}\right]
\end{aligned}
$$

en donde $d \tilde{\Sigma}_{d-1}^{2}$ es una métrica constante de curvatura negativa sobre la variedad de base $\tilde{\Sigma}_{d-1}$. Nótese que los dos bordes conformes desconectados se ubican en $x= \pm 1$.

Para construir los propagadores del borde al volumen $\mathrm{K}^{ \pm}$discutidas antes se propone

$$
\mathrm{K}^{ \pm}\left(t, x, \theta \mid t^{\prime}, \theta^{\prime}\right)=\int_{-\infty}^{\infty} \frac{d \omega}{2 \pi} \sum_{Q} e^{-i \omega\left(t-t^{\prime}\right)} Y_{Q}(\theta) Y_{Q}^{*}\left(\theta^{\prime}\right) f_{\omega Q}^{ \pm}(x)
$$

aquí $Y_{Q}(\theta)$ son funciones armónicas ${ }^{11}$ sobre $\tilde{\Sigma}_{d-1}$.

Insertando (2.54) en las ecuaciones de KG (2.6) se encuentra que $f_{\omega Q}$ satisface

$$
\left(1-x^{2}\right) \frac{d^{2} f_{\omega Q}^{ \pm}(x)}{d x^{2}}+(d-2) x \frac{d f_{\omega Q}^{ \pm}(x)}{d x}+\left[\left(\omega^{2}-Q^{2}\right)-\frac{m^{2} R^{2}}{1-x^{2}}\right] f_{\omega Q}^{ \pm}(x)=0 .
$$

Las soluciones a (2.55) pueden ser escritas en términos de polinomios generalizados de Legendre como [30]

$$
f_{\omega Q}^{ \pm}(x)=\left(1-x^{2}\right)^{\frac{d}{4}}\left[a_{\omega Q}^{ \pm} P_{\nu}^{\mu}(x)+b_{\omega Q}^{ \pm} Q_{\nu}^{\mu}(x)\right]
$$

en donde $\mu$ está dado por (2.5) y

$$
\nu=\varpi-\frac{1}{2}=\sqrt{\left(\frac{d-1}{2}\right)^{2}+\omega^{2}-Q^{2}}-\frac{1}{2} .
$$

$a_{\omega Q}^{ \pm}, b_{\omega Q}^{ \pm}$en (2.56) son coeficientes constantes que quedan fijados cuando imponemos las condiciones (2.31), estos son

$$
f_{\omega Q}^{ \pm}\left( \pm x_{\epsilon}\right)=1, \quad f_{\omega Q}^{ \pm}\left(\mp x_{\epsilon}\right)=0 .
$$

\footnotetext{
${ }^{11}$ Estas funciones satisfacen $\nabla_{\Sigma}^{2} Y_{Q}=-Q^{2} Y_{Q}$ y en una variedad conforme con bordes se tiene $Q^{2} \geq 0$. Los modos propios y autovalores para el Laplaciano sobre una variedad compacta suave hiperbólica no pueden expresarse en una forma cerrada analítica y depende de un subgrupo discreto de $S O(d-1,1)$ elegido para hacer el cociente. Ver [87] y las referencias citadas en él para tener un tratamiento numérico del problema.
} 
Las soluciones para (2.56) que satisfacen (2.58) son

$$
\begin{aligned}
& f_{\omega Q}^{+}(x)=\left(\frac{1-x^{2}}{1-x_{\epsilon}^{2}}\right)^{\frac{d}{4}} \frac{P_{\nu}^{\mu}(x) Q_{\nu}^{\mu}\left(-x_{\epsilon}\right)-P_{\nu}^{\mu}\left(-x_{\epsilon}\right) Q_{\nu}^{\mu}(x)}{P_{\nu}^{\mu}\left(x_{\epsilon}\right) Q_{\nu}^{\mu}\left(-x_{\epsilon}\right)-P_{\nu}^{\mu}\left(-x_{\epsilon}\right) Q_{\nu}^{\mu}\left(x_{\epsilon}\right)} \\
& f_{\omega Q}^{-}(x)=\left(\frac{1-x^{2}}{1-x_{\epsilon}^{2}}\right)^{\frac{d}{4}} \frac{P_{\nu}^{\mu}(x) Q_{\nu}^{\mu}\left(x_{\epsilon}\right)-P_{\nu}^{\mu}\left(x_{\epsilon}\right) Q_{\nu}^{\mu}(x)}{P_{\nu}^{\mu}\left(-x_{\epsilon}\right) Q_{\nu}^{\mu}\left(x_{\epsilon}\right)-P_{\nu}^{\mu}\left(x_{\epsilon}\right) Q_{\nu}^{\mu}\left(-x_{\epsilon}\right)},
\end{aligned}
$$

La existencia de dos propagadores volumen-borde independientes proviene del echo de que la variedad de base $\tilde{\Sigma}$ nunca se reduce a tamaño cero dentro del volumen (ver (2.3)) y entonces la regularidad en el interior no impone ninguna condición sobre las soluciones (2.56). Los modos normalizables aparecen para

$$
\omega_{n Q}= \pm \sqrt{\left(\mu+\frac{1}{2}+n\right)^{2}+Q^{2}-\left(\frac{d-1}{2}\right)^{2}}, \quad n=0,1, \ldots, \quad \text { y } \quad \frac{b_{\omega Q}}{a_{\omega Q}}=-\frac{2 \tan \pi \mu}{\pi}
$$

En estas frecuencias el índice $\nu$ en (2.57) toma el valor $\nu_{n}=\mu+n$ y la solución resultante se hace normalizable. Las funciones de dos puntos (2.35)-(2.36) entre operadores $\mathcal{O}_{ \pm}$sobre el mismo y opuestos bordes toman la forma

$$
\begin{aligned}
\left\langle\psi_{\mathrm{f}}\left|T \mathcal{O}^{ \pm}(t, \theta) \mathcal{O}^{ \pm}\left(t^{\prime}, \theta^{\prime}\right)\right| \psi_{\mathrm{i}}\right\rangle= & \pm i \frac{2^{\Delta_{-}}}{\pi 2^{d}} \frac{\Gamma(1-\mu)}{\Gamma(1+\mu)} \sum_{Q} Y_{Q}(\theta) Y_{Q}^{*}\left(\theta^{\prime}\right) \\
& \times \int \frac{d \omega}{2 \pi} e^{-i \omega\left(t-t^{\prime}\right)} \Gamma\left(\frac{1}{2}+\mu-\varpi\right) \Gamma\left(\frac{1}{2}+\mu+\varpi\right) \cos (\pi \varpi) \\
\left\langle\psi_{\mathrm{f}}\left|T \mathcal{O}^{ \pm}(t, \theta) \mathcal{O}^{\mp}\left(t^{\prime}, \theta^{\prime}\right)\right| \psi_{\mathrm{i}}\right\rangle= \pm & \pm i \frac{2^{\Delta_{-}}}{2^{d-1}} \frac{1}{\Gamma(\mu)^{2}} \sum_{Q} Y_{Q}(\theta) Y_{Q}^{*}\left(\theta^{\prime}\right) \\
& \times \int \frac{d \omega}{2 \pi} e^{-i \omega\left(t-t^{\prime}\right)} \Gamma\left(\frac{1}{2}+\mu-\varpi\right) \Gamma\left(\frac{1}{2}+\mu+\varpi\right)
\end{aligned}
$$

Unos pocos comentarios sobre esta expresión: (i) en el límite de frecuencias grandes $\varpi \sim \omega \mathrm{y}$ los integrandos en (2.61)-(2.62) coinciden con aquellos de $\mathrm{AdS}_{2}$ (confrontar con (2.47)-(2.48)), (ii) cuando se elige el contorno de Feynman, debe entenderse el lado derecho de (2.61)-(2.62) como ordenado temporalmente y (iii) aunque las funciones Gamma $\Gamma\left(\frac{1}{2}+\mu \pm \varpi\right)$ presentan dos cortes en $\omega= \pm \sqrt{Q^{2}-\left(\frac{d-1}{2}\right)^{2}}$ (ver (2.57)), el producto de los integrandos (2.61)-(2.62) está libre de ellos.

La correlación entre operadores insertados sobre bordes opuestos es no nula, y este resultado ha sido explicado de diferentes maneras dependiendo del contexto: (i) como resultado de calcular el correlador (2.62) sobre un estado entrelazado de dos teorías de borde no interactuantes (contexto de agujero negro [31]) o (ii) como debido a una interacción entre teorías definidas sobre cada uno de los bordes (orbifold D1/D5 en [33]). El punto crucial en ambos argumentos fue la ausencia/existencia de conexión causal entre las regiones asintóticas. 


\subsection{Entrelazamiento vs. Acoplamiento}

En esta sección se van a revisar la interpretación de los resultados (2.51) y (2.62). Se intenta responder cuándo son consecuencia de: (i) interacción entre las dos QFT duales o (ii) correladores evaluados en un estado entrelazado o (iii) ambas situaciones.

\section{Entropía de Entrelazamiento}

La entropía de entrelazamiento $S_{A}$ es una cantidad no local que mide cuan correlacionados están dos sistemas, digamos A y B. Para una QFT en $d$ dimensiones se define como la entropía de von Neumann de la matriz densidad reducida $\rho_{A}$ obtenida cuando se toma la traza sobre los grados de libertad correspondientes a una sub-variedad tipo espacio de $d-1$ dimensiones, llamada $\mathrm{B}$, que es el complemento de A (ver [85] para un resumen).

En $[82,83]$ se propuso una formula holográfica para la entropía de entrelazamiento de una $\mathrm{CFT}_{d}$ dual a una geometría $\mathrm{AdS}_{d+1}$, esta es

$$
\mathrm{S}_{\mathrm{A}}=\frac{\operatorname{Area}\left(\gamma_{\mathrm{A}}\right)}{4 G_{N}^{(d+1)}}
$$

en donde $\gamma_{\mathrm{A}}$ es un area mínima $(d-1)$ dimensional en $\operatorname{AdS}_{d+1}$ cuyo borde $\mathcal{S}$, ubicado en el infinito de AdS, coincide con el de A, esto es $\mathcal{S}=\partial \gamma_{\mathrm{A}}=\partial \mathrm{A}$ y $G_{N}^{(d+1)}$ es la constante de Newton en $\mathrm{AdS}_{d+1}$. Esta fórmula, que supone la aproximación de supergravedad de la teoría de cuerdas completa, a sido aplicada en el caso de $\mathrm{AdS}_{3} / \mathrm{CFT}_{2}$ mostrando perfecto acuerdo con los resultados conocidos de la 2D CFT [85].

Podemos aplicar una generalización de (2.63) a la geometría de agujero de gusano (2.3) como sigue ${ }^{12}$ : el agujero de gusano presenta dos regiones espaciales desconectadas $\Sigma_{ \pm}$en las cuales se supone que viven dos grados de libertad idénticos. Imaginemos que construimos una superficie cerrada de codimension 1 que es el borde, $\mathcal{S}$, de una pequeña región B sobre $\Sigma_{-}$, experiencia en lazos de Wilson y embeddings de branas muestra que mientras B permanezca chico, la superficie mínima se ubicará cerca de $x=-1$. A medida que vamos incrementando el tamaño de $\mathrm{B}$, la superficie mínima extendida sobre $\Sigma_{-}$explora regiones más profundas del volumen del espacio y en el límite cuando $\mathrm{B} \rightarrow \Sigma_{-}$el borde $\mathcal{S}$ colapsa y la superficie mínima se localiza en la garganta $x=0$ de la geometría (ver figura 2.3), ofreciendo un resultado no nulo

$$
\mathrm{S}_{\tilde{\Sigma}}=\frac{\operatorname{Area}(\tilde{\Sigma})}{4 G_{N}^{(d+1)}}
$$

Este resultado debe entenderse como un indicador de que el estado cuántico en la QFT dual descripto por la geometría de agujero de gusano es no separable, o dicho de otra manera, el

\footnotetext{
${ }^{12}$ Ver [84] para obtener una generalización de (2.63) para el agujero de gusano Euclídeo construido en [25].
} 


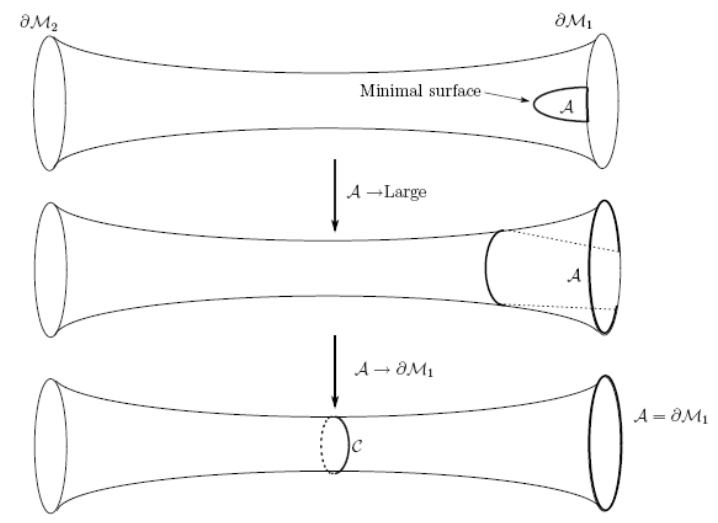

Figura 2.3: Área mínima de la superficie cuyos extremos se encuentran sobre el borde de la región $\mathcal{A}$. A medida que $\mathcal{A}$ va cubriendo todos los grados de libertad de una de las teorías de campos, la superficie mínima explora el interior del agujero de gusano. Cuando $\mathcal{A}$ cubre todos los grados de libertad $\left(\mathcal{A}=\partial \mathcal{M}_{1}\right)$ la superficie mínima es el área del cuello.

resultado (2.62) puede ser atribuido a que la geometría de agujero de gusano realiza un estado entrelazado en el espacio de Hilbert producto $\mathcal{H}=\mathcal{H}_{+} \otimes \mathcal{H}_{-}$con la entropía (2.64) resultante de integrar los grados de libertad sobre $\mathcal{H}_{-}$.

\section{Interacción entre copias duales}

Se argumentará que además del entrelazamiento discutido anteriormente, el contacto causal entre los bordes asintóticos de la geometría de agujero de gusano conduce a un término de interacción entre los dos conjuntos de grados de libertad que viven en cada uno de los bordes. En particular, se concluirá que existe un acoplamiento entra las teorías de campos cuando las regiones asintóticas se encuentren en contacto causal.

Comenzaré escribiendo la función de partición en el lado de gravedad para la geometría de agujero de gusano en el límite semiclásico, su forma es

$$
\mathcal{Z}_{\text {gravity }}\left[\phi\left(\phi_{0}^{+}, \phi_{0}^{-}, \mathcal{C}\right)\right] \sim e^{-\frac{i}{2} \int d \mathbf{y} d \mathbf{y}^{\prime} \phi_{0}^{i}(\mathbf{y}) \Delta_{i j}\left(\mathbf{y}, \mathbf{y}^{\prime}\right) \phi_{0}^{j}\left(\mathbf{y}^{\prime}\right)}
$$

aquí $\mathbf{y}=(t, \theta)$ denota los puntos en el borde y $i, j=+,-$ refieren a los bordes asintóticos en donde el dato $\phi_{0}^{ \pm}$es dado, el contorno $\mathcal{C}$ fija las soluciones normalizables en el volumen del espacio y la expresión para $\Delta_{i j}$ esta dada por (2.34). De acuerdo a la prescripción GKPW la función de partición (2.65) es la funcional generatriz para correladores $\mathcal{O}^{ \pm}$, esto es

$$
\mathcal{Z}_{\text {gravity }}\left[\phi\left(\phi_{0}^{+}, \phi_{0}^{-}, \mathcal{C}\right)\right]=\left\langle\psi_{\mathrm{f}}\left|T e^{i \int d \mathbf{y} \phi_{0}^{+}(\mathbf{y}) \mathcal{O}^{+}(\mathbf{y})+i \int d \mathbf{y} \phi_{0}^{-}(\mathbf{y}) \mathcal{O}^{-}(\mathbf{y})}\right| \psi_{\mathrm{i}}\right\rangle_{Q F T},
$$

en donde los observables $\mathcal{O}^{ \pm}$deben ser construidos como funcionales locales de los campos fundamentales $\Psi_{ \pm}$definidos en cada uno de los bordes. Se asume que estos campos describen grados de libertad independientes: $\left[\mathcal{O}^{+}, \mathcal{O}^{-}\right]=0$ sobre la misma rebanada espacial (ver pie de página 10 ). 
Consideremos la situación más simple correspondiente a elegir a $\mathcal{C}$ como el contorno de Feynman, esto es, se están calculando las amplitudes de transición vacío-vacío en la teoría de campos. El lado derecho en (2.66) puede escribirse como

$\left\langle\psi_{0}\left|T e^{i \int d \mathbf{y} \phi_{0}^{+}(\mathbf{y}) \mathcal{O}^{+}(\mathbf{y})+i \int d \mathbf{y} \phi_{0}^{-}(\mathbf{y}) \mathcal{O}^{-}(\mathbf{y})}\right| \psi_{0}\right\rangle_{Q F T}=\operatorname{Tr}\left[\rho_{\psi_{0}} T e^{i \int d \mathbf{y} \phi_{0}^{+}(\mathbf{y}) \mathcal{O}^{+}(\mathbf{y})+i \int d \mathbf{y} \phi_{0}^{-}(\mathbf{y}) \mathcal{O}^{-}(\mathbf{y})}\right]$,

en donde ya se a realizado la operación de traza sobre un conjunto completo de estados del espacio de Hilbert de la teoría de campos dual y $\rho_{\psi_{0}}=\left|\psi_{0}\right\rangle\left\langle\psi_{0}\right|$ es la matriz densidad asociada al estado de vacío de agujero de gusano. Este estado de vacío pertenece al espacio de Hilbert $\mathcal{H}=\mathcal{H}_{+} \otimes \mathcal{H}_{-}$y de acuerdo con los argumentos resumidos en la subsección anterior, no es separable como un único producto tensorial $\left|\psi_{0}\right\rangle \neq\left|\psi_{+}\right\rangle \otimes\left|\psi_{-}\right\rangle$.

Para analizar la posibilidad de interacción entre los campos definidos en cada uno de los bordes consideremos el sistema a temperatura finita $T=\beta^{-1}$. La ausencia de singularidades en la continuación Euclídea implica que el agujero de gusano esta en equilibrio con un reservorio térmico de temperatura arbitraria, o dicho de otra manera, la periodicidad en el tiempo Euclídeo es arbitraria. En equilibrio térmico un estado de la teoría de campos queda descrito por la distribución de Boltzmann $\rho_{\beta}=e^{-\beta H}$, con $H=H_{+}\left[\Psi_{+}\right]+H_{-}\left[\Psi_{-}\right]+H_{\text {int }}\left[\Psi_{+}, \Psi_{-}\right]$el Hamiltoniano de la teoría de campos dual, y $H_{\text {int }}$ un posible término de acoplamiento entre los conjuntos idénticos de grados de libertad $\Psi_{ \pm}$.

Ahora veremos que el término de interacción $H_{\text {int }}$ debe estar presente en el Hamiltoniano para evitar una contradicción. El argumento es el siguiente: las funciones de correlación a temperatura finita sobre la teoría de campos son obtenidas a partir de una rotación Euclídea de (2.67), con lo cual

$$
\left\langle e^{-\int d \mathbf{y} \phi_{0}^{+}(\mathbf{y}) \mathcal{O}^{+}(\mathbf{y})-\int d \mathbf{y} \phi_{0}^{-}(\mathbf{y}) \mathcal{O}^{-}(\mathbf{y})}\right\rangle_{\beta}=\operatorname{Tr}\left[\rho_{\beta} e^{-\int d \mathbf{y} \phi_{0}^{+}(\mathbf{y}) \mathcal{O}^{+}(\mathbf{y})-\int d \mathbf{y} \phi_{0}^{-}(\mathbf{y}) \mathcal{O}^{-}(\mathbf{y})}\right],
$$

la teoría de campos esta definida sobre una variedad Euclídea con topología $S_{\beta}^{1} \times \tilde{\Sigma}$ (ver pie de página 10). La propuesta de AdS/CFT iguala esta expresión con la continuación Euclídea del lado izquierdo de (2.66), el resultado es

$$
\operatorname{Tr}\left[e^{-\beta H} e^{-\int d \mathbf{y} \phi_{0}^{+}(\mathbf{y}) \mathcal{O}^{+}(\mathbf{y})-\int d \mathbf{y} \phi_{0}^{-}(\mathbf{y}) \mathcal{O}^{-}(\mathbf{y})}\right]=\mathcal{Z}_{\text {Egravity }}\left[\phi\left(\phi_{0}^{+}, \phi_{0}^{-}\right)\right] \sim e^{-S_{E}\left[\phi_{0}^{+}, \phi_{0}^{-}\right.} \gamma_{2}
$$

sobre el lado derecho $S_{E}\left[\phi_{0}^{+}, \phi_{0}^{-}\right]$corresponde a la acción para el campo escalar sobre el fondo gravitatorio del agujero de gusano evaluada en la solución de las ecuaciones de movimiento para dicho campo con el tiempo compactificado sobre un circulo de radio $\beta$. Luego de la rotación Euclídea y la compactificación temporal, la geometría resultante es un cilindro con topología $S_{\beta}^{1} \times I \times \tilde{\Sigma}$ y los dos bordes sobre los cuales se imponen los datos $\phi_{0}^{ \pm}$se localizan en los extremos $x_{ \pm}$del intervalo 
finito $I^{13}$. Nótese que en el contexto Euclídeo la solución en el interior queda completamente especificada por los datos del borde, existen soluciones no normalizables y rotar el contorno de Feynman al eje temporal imaginario conduce a soluciones no singulares. La expresión explícita para el lado derecho de (2.69) es

$$
\mathcal{Z}_{\text {Egravity }}\left[\phi\left(\phi_{0}^{+}, \phi_{0}^{-}\right)\right] \sim e^{-\frac{1}{2} \int d \mathbf{y} d \mathbf{y}^{\prime} \phi_{0}^{i}(\mathbf{y}) \tilde{\Delta}_{i j}\left(\mathbf{y}, \mathbf{y}^{\prime}\right) \phi_{0}^{j}\left(\mathbf{y}^{\prime}\right)}
$$

en donde $\tilde{\Delta}_{i j}$ denota el propagador volume-borde (2.34) rotado de manera Euclídea. Finalmente, a partir de (2.69) y (2.70) se obtiene la prescripción gauge/gravedad para una agujero de gusano a temperatura finita

$$
\operatorname{Tr}\left[e^{-\beta H} e^{-\int d \mathbf{y} \phi_{0}^{+}(\mathbf{y}) \mathcal{O}^{+}(\mathbf{y})-\int d \mathbf{y} \phi_{0}^{-}(\mathbf{y}) \mathcal{O}^{-}(\mathbf{y})}\right] \sim e^{-\frac{1}{2} \int d \mathbf{y} d \mathbf{y}^{\prime} \phi_{0}^{i}(\mathbf{y}) \tilde{\Delta}_{i j}\left(\mathbf{y}, \mathbf{y}^{\prime}\right) \phi_{0}^{j}\left(\mathbf{y}^{\prime}\right)} .
$$

Esta es la fórmula central del argumento debido a que si uno asume que los grados de libertad $\Psi_{+}, \Psi_{-}$son desacoplados, esto es

$$
H\left[\Psi_{+}, \Psi_{-}\right]=H_{+}\left[\Psi_{+}\right]+H_{-}\left[\Psi_{-}\right]
$$

luego, $\rho_{\beta}=e^{-\beta H_{+}} e^{-\beta H_{-}}$, y el lado izquierdo de (2.71) se factoriza en un producto de dos cantidades: una que depende de $\phi_{0}^{+}$y otra que depende de $\phi_{0}^{-14}$. Sin embargo, el cálculo de gravedad no se factoriza debido a los términos no nulos $\tilde{\Delta}_{ \pm \mp}$. Se interpreta este resultado como la manifestación de existencia de un acoplamiento no trivial $H_{i n t}$ entre los grados de libertad duales $\Psi_{ \pm}$: la teoría de campos dual a la geometría de agujero de gusano contiene un término de acoplamiento no trivial entre los grados de libertad de sus bordes $\Psi_{+}$y $\Psi_{-}$.

\section{Aplicaciones}

El resultado de las observaciones anteriores es que la geometría de agujero de gusano codifica la descripción de una teoría de campos dual consistente de dos copias de campos fundamentales en interacción. Además, el estado cuántico descripto por el agujero de gusano está entrelazado. En particular, la continuación Euclídea puede ser vista como prescripción para separar, en la teoría de campos dual, efectos de entrelazamiento y de interacción $H_{i n t}$. Una función de correlación no nula entre operadores localizados en bordes diferentes debe interpretarse como originados a partir de una interacción $H_{i n t}$, en lugar de ser vistos como producido por efectos de entrelazamiento. En lo que sigue se confrontarán estos dos puntos de vista con otras dos geometrías relevantes en la literatura: $\mathrm{AdS}_{1+1}$ y el agujero negro eterno de $\mathrm{AdS}$ [31].

\footnotetext{
${ }^{13}$ Nótese las similitud con la geometría estudiada en [25].

${ }^{14}$ Nótese que en ausencia de interacción $\left[\mathcal{O}_{+}, \mathcal{O}_{-}\right]=0$ sin importar si los puntos en donde están insertados los operadores están espaciados temporal/espacialmente.
} 


\section{$\operatorname{AdS}_{1+1}:$}

$\mathrm{El}$ análisis de $\mathrm{AdS}_{1+1}$ (2.39) es enteramente análogo al agujero de gusano estudiado. La sección Euclídea de la métrica global (2.39) tiene dos bordes antes de compactificar la dirección temporal y por lo tanto se requieren dos condiciones de borde $\phi_{0}^{ \pm}$en el límite semiclásico (ver (2.65)). Los argumentos que conducen a (2.71) se aplican con las funciones de correlación Euclídeas $\tilde{\Delta}_{i j}$ inmediatamente obtenidas a partir de las fórmulas Lorentzianas (2.51). Se concluye entonces que el echo de tener $\tilde{\Delta}_{ \pm \mp} \neq 0$ nos dice que $\mathrm{AdS}_{1+1}$ es dual a una mecánica cuántica conforme compuesta de dos sectores interactuantes.

\section{Agujero negro eterno de AdS:}

La diferencia crucial entre el agujero de gusano (2.3) y la solución de agujero negro de AdS máximamente extendida [31] es la siguiente: al realizar la continuación Euclídea de la solución de agujero negro, la existencia de un horizonte en signatura Lorentziana genera una singularidad cónica en la variedad Euclídea, que solo puede ser evitada pidiendo una periodicidad precisa en el tiempo Euclídeo. La geometría Riemanniana resultante tiene inevitablemente solo un borde asintótico, y entonces requiere imponer solo un dato en el borde, $\phi_{0}$, esto indica la existencia de un único conjunto de grados de libertad $\Psi$ y un único propagador volumen-borde $\tilde{\Delta}$. El sistema en equilibrio con un baño térmico de temperatura fija (determinada por la masa del agujero negro) tiene una funcional generatriz que se escribe

$$
\operatorname{Tr}\left[e^{-\beta H} e^{-\int d \mathbf{y} \phi_{0}(\mathbf{y}) \mathcal{O}(\mathbf{y})}\right] \sim e^{-\frac{1}{2} \int d \mathbf{y} d \mathbf{y}^{\prime} \phi_{0}(\mathbf{y}) \tilde{\Delta}\left(\mathbf{y}, \mathbf{y}^{\prime}\right) \phi_{0}\left(\mathbf{y}^{\prime}\right)},
$$

La solución en tiempo real (agujero negro máximamente extendido) fue analizada e interpretada en el contexto de AdS/CFT en [31]. El segundo borde causalmente desconectado, presente en signatura Lorentziana, fue entendido como soportando los acompañantes de TFD (dinámica de campos térmicos) necesarios para obtener un estado térmico a partir de su integración y conduce a una duplicación del espacio de Hilbert $\mathcal{H}=\mathcal{H}_{+} \otimes \mathcal{H}_{-}$. El segundo conjunto de grados de libertad está causalmente desconectado del conjunto original a temperatura cero; aunque ellos aparezcan en signatura Lorentziana y den lugar a funciones $\Delta_{i j}$ no triviales, ellos son ficticios desde el punto de vista físico. La desconexión causal entre los bordes está relacionada con que la función de partición de dos puntos $\Delta_{ \pm \mp}$ nunca es singular.

\subsection{Conclusiones}

En este capítulo se resumió la prescripción GKPW en el contexto Lorentziano relacionando la ambigüedad de agregar modos normalizables a (2.3) con el contorno de integración $\mathcal{C}$ requerido 
para evitar las singularidades en el integrando de (2.27), (2.61) proviene de la existencia de modos normalizables. Para calcular cantidades Lorentzianas se necesita fijar un contorno de referencia $\mathcal{C}_{\text {ref }}$ y las dos elecciones sensibles son la retardada o la de Feynman. Estas elecciones son duales, en el lado de la QFT, a calcular funciones de respuesta o de correlación. Se eligió el camino de Feynman como contorno de referencia, cualquier otro contorno $\mathcal{C}$ difiere de $\mathcal{C}^{F}$ en contribuciones que provienen de enlazar polos, estos polos enlazados fijan los estados inicial y final en la función de correlación (ver (2.16)).

En la sección 2.2 se extendió la prescripción de GKPW a espacio-tiempos con más de un borde asintótico, en particular se estudió el caso de dos bordes. Se propuso escribir el campo en el volumen del espacio en términos de dos valores de borde asintóticos y como ejemplo de juguete se aplicó esta construcción al caso $\mathrm{AdS}_{1+1}$ reobteniendo resultados previos, después se calcularon las funciones de correlación de dos puntos para el agujero de gusano de Einstein-Gauss-Bonnet (2.3). En este punto debo enfatizar que la diferencia principal entre este método y el utilizado en [33], para un orbifolio de $\mathrm{AdS}_{3}$, consiste en que hemos considerado los valores de borde $\phi_{0}^{ \pm}$del campo escalar en el volumen como cantidades independientes, además, se mostró explícitamente la posibilidad de construir dos propagadores volumen-borde $\mathrm{K}^{ \pm}$(su condición de borde fue dada en (2.31)). La construcción realizada en [33] utilizó una relación entre los valores de borde $\phi_{0}^{ \pm}$y esto fue entendido como un indicativo de que las fuentes \pm para la teoría de campos dual están prendidas de manera correlacionada. Una cuestión que permanece pendiente es cuándo los dos conjuntos de datos son independientes o redundantes en la formulación dual, por otro lado otra cuestión es el origen de valores no nulos para operadores ubicados en bordes diferentes $\left(\Delta_{ \pm \mp}\right)$ esto puede ser producido por entrelazamiento o por interacciones o ambos casos simultáneamente.

En la sección 2.3 se aplicaron las ideas de entrelazamiento holográfico desarrolladas en [83] a la geometría de agujero de gusano. El resultado no nulo obtenido para $\mathrm{S}_{\tilde{\Sigma}}$ de los grados de libertad que viven sobre bordes opuestos sugieren que el agujero de gusano debe ser entendido como representando a un estado entrelazado en $\mathcal{H}=\mathcal{H}_{+} \otimes \mathcal{H}_{-}$. Por otro lado, la conexión causal entre los bordes sugiere que también puede existir un acoplamiento. Para atacar este problema se consideró al sistema del agujero de gusano en contacto con un baño térmico, después de la Euclideanización, la geometría resultante aún tiene dos bordes, esto indica, para evitar una contradicción, que la QFT dual consiste de dos copias $\mathcal{H}_{ \pm}$en interacción. En resumen, el número de bordes desconectados de la sección Euclídea determina la cantidad de grados de libertad físicos.

Me gustaría enfatizar finalmente las implicaciones de este trabajo sobre la gravedad cuántica, la cual puede ser vista como una de las motivaciones. Este tema a sido discutido en diferentes contextos en los últimos años y es referido como espacio-tiempo emergente [86]. En este sentido, se mostró cómo se decodifican propiedades topológicas y causales (conectividad) en la acción de 
la QFT, y esta parte de la información no está solamente en el estado fundamental sino en su estructura de interacciones. Espero que esta conclusión pueda contribuir a la construcción de reglas hacia una ingeniería de geometrías.

Debo mencionar que en presencia de interacciones se necesita decir cómo están identificados los puntos sobre bordes opuestos. Un primer paso hacia el entendimiento de este problema es identificar los puntos $\mathbf{y}, \mathbf{y}^{\prime}$ en el espacio de configuración en los cuales $\Delta_{ \pm \mp}$ diverge y estudiar la consistencia de identificarlos, esto esta siendo investigado actualmente. 


\section{Capítulo 3}

\section{Estabilidad de lazos de Wilson}

En los últimos años, la correspondencia gauge/gravedad [1, 4, 5] nos permitió estudiar nuevos aspectos referidos al fenómeno de confinamiento. El punto crucial es que el aspecto de cuerda que presenta el tubo de flujo cromoeléctrico se manifiesta en la teoría dual mediante la aparición de una dimensión holográfica $[88,89]$. En $[45,46]$ se propuso desde la teoría de cuerdas una prescripción para calcular el valor de expectación del lazo de Wilson: el lazo de la teoría de gauge se puede pensar localizado en infinito en la coordenada radial holográfica y el lazo de Wilson para quarks fundamentales queda definido por una cuerda abierta cuyos extremos están sobre él. En el límite de $N_{c}$ grande (límite de 't Hooft) las auto-interacciones de la cuerda de QCD se anulan [2] y la prescripción gravitatoria dual para calcular la energía potencial entre quarks en una dada teoría de gauge queda reducida a encontrar una superficie mínima en la teoría de gravedad.

Como se explicó en la sección 1.1.3, el cálculo canónico de la energía potencial entre un par de quarks fundamentales (para un lazo rectangular), involucra una cuerda con forma de U extendida en la dirección holográfica, en donde la separación, $L$, de los quarks en la teoría de gauge se traduce en la separación entre los extremos fijos de la cuerda ubicados en infinito. A medida que varía la separación entre los quarks la cuerda explora la dirección holográfica, luego, la posición radial mínima alcanzada por la cuerda, $r_{0}$, depende de la distancia de separación entre los extremos L. Este procedimiento ha sido aplicado a un gran número de ejemplos paradigmáticos y a dado resultados consistentes con lo que es esperable obtener de la teoría de gauge, en particular, existe un teorema que establece condiciones suficientes para obtener fondos gravitatorios confinantes [91] (ver [90] para un resumen). Extensiones a representaciones más altas del grupo de gauge y lazos de 't Hooft han sido analizadas y propuestas en [92, 93, 94, 95]. Estas, involucran D-branas de dimensiones más altas con y sin campos de gauge prendidos respectivamente.

En muchas aplicaciones a fondos gravitatorios duales, la prescripción $[45,46]$ ha sido aplicada a orden cero para establecer confinamiento, transiciones de fase o propiedades de transporte [90, 96], solo recientemente ha sido estudiada la estabilidad de algunas formas de embeber las cuerdas 
clásicas en el fondo de gravedad [97]-[101] (ver también [104]-[107]). Una de las motivaciones para el análisis de la estabilidad, en general, ha sido la existencia de multiples formas de embeber la cuerda en el espacio de fondo para unas dadas condiciones de contorno [98]-[101],[108] (ver también [109]) señaladas por la presencia de un extremo en la función longitud $L\left(r_{0}\right)$ (ver [110], [111]). La presencia de una longitud de separación máxima fue interpretada como el fenómeno dual a la presencia de apantallamiento en la teoría de gauge. El motivo del presente trabajo es mostrar que aquellas ramas de la solución con $L^{\prime}\left(r_{0}\right)>0$ son inestables. Se confirmará esta afirmación mostrando explícitamente la existencia, en fondos de gravedad particulares, de modos inestables $\left(\omega^{2}<0\right)$ para la rama con $L^{\prime}\left(r_{0}\right)>0$. Es oportuno mencionar que este es un resultado satisfactorio, ya que la forma físicamente aceptable de la función $L\left(r_{0}\right)$ calculada desde la correspondencia gauge/gravedad esta dada por $L^{\prime}\left(r_{0}\right)<0$. Voy a mencionar brevemente cálculos de lazos de 't Hooft en duales gravitatorios con dichas inestabilidades.

En el camino, se discutirán varios aspectos de las diferentes formas de embeber una cuerda en el espacio de fondo: el primer análisis estará relacionado a la interpretación física de la configuración empleada para obtener una energía potencial finita entre los quarks en duales gravitatorios suaves, la segunda trata sobre la invarianza ante difeomorfismos de la acción de la cuerda y su relación con las posibles elecciones de gauge para la orientación de las fluctuaciones en un plano, la tercera es la relación entre las inestabilidades producida por la forma de embeber la cuerda y $L\left(r_{0}\right)$, es decir, la relación entre la separación de los extremos de la cuerda como función de la profundidad máxima alcanzada por la misma en la dirección holográfica. En [99, 100, 101] se demostró que la presencia de un extremo en $L\left(r_{0}\right)$ es un indicativo de la existencia de un modo cero en las fluctuaciones longitudinales, lo cual señala la presencia de una inestabilidad. Voy a confirmar esta echo calculando explícitamente los modos de fluctuación más bajos en diferentes duales gravitatorios.

En este capítulo se estudiará la estabilidad de soluciones clásicas para la hoja de mundo de una cuerda, la cual se emplea para calcular la energía potencial entre quarks fundamentales en duales gravitatorios confinantes y no confinantes. Se discutirá el fijado ante difeomorfismos de la acción de la cuerda, su relación con la orientación de la fluctuación y la interpretación de la sustracción de la masa de los quarks necesaria para calcular la energía potencial en fondos de gravedad (confinantes) suaves. Voy a estudiar varios duales gravitatorios y mostraré mediante análisis numérico la existencia de inestabilidades ante fluctuaciones lineales para soluciones clásicas de cuerdas que tienen derivada positiva de su función longitud $L^{\prime}\left(r_{0}\right)>0$. Finalmente se discutirán brevemente los lazos de 't Hooft en fondos gravitatorios no conformes.

El capítulo estará organizado de la siguiente manera: en la sección 3.1 voy a resumir la prescripción para calcular valores de expectación de lazos de Wilson desde el punto de vista del dual gravitatorio. En la sección 3.2 se describirán los fondos de gravedad bajo estudio y se calcularán las 
funciones de longitud y energía. En la sección 3.3 se van a estudiar fluctuaciones a orden cuadrático y se calcularán numéricamente los modos mas bajos de fluctuación. En la sección 3.4 se analizarán los resultados obtenidos en la sección 3.3 llevando las ecuaciones de movimiento para las fluctuaciones a ecuaciones de Schrodinger. En la sección 3.5 se va a discutir brevemente el lazo de 't Hooft y en las sección 3.6 se estudiarán fluctuaciones bosónicas y fermiónicas en el dual gravitatorio de un operador de Wilson-’t Hooft evaluado en la representación simétrica del grupo de gauge.

\subsection{Soluciones de Cuerda y Lazos de Wilson}

\subsubsection{Cuerdas estáticas}

El punto de partida para realizar cálculos de valores de expectación de lazos de Wilson, en el límite de $\mathrm{N}_{c}, \lambda$ grandes (límite de 't Hooft), a partir de duales gravitatorios con métrica $g_{\mu \nu}$ es la acción de Nambu-Goto ${ }^{1}$

$$
S=\frac{\eta}{2 \pi \alpha^{\prime}} \int d \tau d \sigma \sqrt{\eta h} .
$$

donde $h=\operatorname{det} h_{\alpha \beta}, h_{\alpha \beta}=g_{\mu \nu} \partial_{\alpha} X^{\mu} \partial_{\beta} X^{\nu}$ es la métrica inducida sobre la hoja de mundo de la cuerda, $\partial_{\alpha}=\partial / \partial \xi^{\alpha}$ con $\xi^{\alpha}=\{\tau, \sigma\}$ son las coordenadas sobre la hoja de mundo y $X^{\mu}$ tomando valores sobre el espacio de fondo (target). El signo $\eta$ da cuenta de posibles configuraciones Euclídeas $(\eta=+)$ o Lorentzianas (tipo tiempo) $(\eta=-)$. Las clases de métricas que se van a considerar son de la forma

$$
d s^{2}=-g_{t}(r) d t^{2}+g_{x}(r) d x_{i}^{2}+g_{r}(r) d r^{2}+g_{a b}(r, \theta) d \theta^{a} d \theta^{b} .
$$

Las coordenadas $t, x_{i}(i=1,2,3)$ representan las coordenadas sobre la teoría de gauge, $r$ es la coordenada holográfica y los $\theta_{a}(a, b=1, . ., 5)$ son coordenadas angulares adicionales que parametrizan un espacio compacto 5-dimensional $\Sigma_{5}$. Para conocer la energía potencial entre los quarks se debe resolver la acción de NG para hojas de mundo (tipo tiempo) correspondientes a cuerdas cuyos extremos en infinito se localizan sobre el lazo a calcular, típicamente los extremos están separados por una distancia $L$ en una de las coordenadas $x_{i}$ a la cual llamaré $x$ (ver [90]). Voy a comenzar analizando formas estáticas de embeber la cuerda $t(\tau), x(\sigma), r(\sigma)$, con todas las otras coordenadas fijadas a constantes ${ }^{2}$. Las ecuaciones de movimiento correctas se obtienen de insertar la propuesta de solución (ansatz) en la acción, se tiene entonces

\footnotetext{
${ }^{1}$ En general uno debería tener en cuenta contribuciones de los campos de fondo $B_{2}$, no obstante como propuesta de solución se va a considerar que no contribuye a (3.1).

${ }^{2}$ Generalizaciones que consideran quarks en movimiento en el borde, relevantes para aplicaciones a plasmas de quarks y gluones (QGP) son refraseadas en términos de componentes no diagonales en la métrica [98],[108].
} 


$$
\begin{aligned}
S & =-\frac{1}{2 \pi \alpha^{\prime}} \int d \tau d \sigma \sqrt{g_{t}(r) \dot{t}^{2}\left(g_{x}(r) \dot{x}^{2}+g_{r}(r) \dot{r}^{2}\right)} \\
& =-\frac{1}{2 \pi \alpha^{\prime}} \int d t d \sigma \sqrt{g_{t}(r)\left(g_{x}(r) \dot{x}^{2}+g_{r}(r) \dot{r}^{2}\right)} \\
& =-\frac{\mathcal{T}}{2 \pi \alpha^{\prime}} \int d \sigma \sqrt{f^{2}(r) \dot{x}^{2}+g^{2}(r) \dot{r}^{2}} .
\end{aligned}
$$

donde $g^{2}(r)=g_{t}(r) g_{r}(r)$ y $f^{2}(r)=g_{t}(r) g_{x}(r)$. La invarianza ante reparametrizaciones de (3.1) factoriza la extensión temporal del lazo $\mathcal{T}$ y reduce el cálculo del lazo de Wilson a encontrar una geodésica en la geometría efectiva 2- dimensional

$$
d s_{\text {eff }}^{2}=f^{2}(r) d x^{2}+g^{2}(r) d r^{2} .
$$

La carga conservada asociada a traslaciones en $x$ en (3.3) es

$$
\frac{f^{2}(r) \dot{x}(\sigma)}{\sqrt{f^{2}(r) \dot{x}(\sigma)^{2}+g^{2}(r) r^{\prime}(\sigma)^{2}}}=A
$$

de donde se obtiene

$$
\dot{x}(\sigma)= \pm A \frac{g(r)}{f(r)} \frac{1}{\sqrt{f^{2}(r)-A^{2}}} \dot{r}(\sigma) .
$$

La invarianza ante reparametrizaciones garantiza que (3.6) resuelve la ecuación de movimiento en la dirección $r$. Nombrando $r_{0}$ al punto dado por $f\left(r_{0}\right)=A$, (3.6) puede reescribirse como [91]

$$
\frac{d x}{d r}= \pm \frac{g(r)}{f(r)} \frac{f\left(r_{0}\right)}{\sqrt{f^{2}(r)-f^{2}\left(r_{0}\right)}}
$$

Las condiciones de borde en infinito para la separación de los extremos de la cuerda son $\left.\Delta x\right|_{r=\infty}=L$. A partir de (3.7) se nota que la cuerda alcanza el borde de manera ortogonal. Hay dos elecciones de gauge naturales que se encuentran en la literatura: $x(\sigma)=\sigma$ (x-gauge) o $r(\sigma)=\sigma$ ( $r$-gauge). La primera elección ( $x$-gauge) tiene el beneficio de proveernos una parametrización completa de $r(x)$ cuando se imponen $x \in[-L / 2, L / 2]$ y $r( \pm L / 2)=\infty$ (la localización de la punta (tip) de la cuerda se escoge en $\left.(r, x)=\left(r_{0}, 0\right)\right)$. Haciendo $x \rightarrow t$, la ecuación (3.7) puede ser entendida como un movimiento de energía cero en un potencial $U(r)$ dado por

$$
U(r)=\frac{f^{2}(r)\left(f^{2}(r)-f^{2}\left(r_{0}\right)\right)}{g^{2}(r) f^{2}\left(r_{0}\right)}
$$

en donde el punto $r_{0}$ puede verse como el valor mínimo alcanzado por la cuerda en la coordenada holográfica. La segunda elección (r-gauge) da una función $x(r)$ bivaluada cuando se impone $r \in$ $\left[r_{0}, \infty\right)$ y $x(\infty)= \pm L / 2$. Sin embargo, existen una gran cantidad de ejemplos que conducen a 
una expresión analítica cerrada para $x(r)$. Además, cuando se calculan fluctuaciones alrededor de soluciones estáticas, se simplifican drásticamente las ecuaciones de movimiento debido a que no contribuyen las fluctuaciones provenientes de las componentes de la métrica $g_{\mu \nu}$ (ver [91],[99, 100, 101]). Se debe tener en cuenta que el tip de la cuerda es un punto especial en el gauge $r$ ya que en dicho punto deben pegarse correctamente las dos ramas correspondientes a los signos \pm en (3.7) (ver en la sección siguiente).

Integrando (3.6) se puede escribir la longitud $L\left(r_{0}\right)$ como,

$$
L\left(r_{0}\right)=2 \int_{r_{0}}^{\infty} \frac{g(r)}{f(r)} \frac{f\left(r_{0}\right)}{\sqrt{f^{2}(r)-f^{2}\left(r_{0}\right)}} d r .
$$

Asumiendo que $f(r), f^{\prime}(r)>0$ el límite de integración inferior $r_{0}$ en (3.9) es, en general, integrable ${ }^{3}$. Nótese que $L\left(r_{0}\right)$ finito en el lado izquierdo de (3.9) demanda que $g / f^{2}$ decaiga en infinito más rápidamente que $\frac{1}{r}$. En las siguientes secciones voy a analizar más profundamente la relación (3.9). La derivada $L^{\prime}\left(r_{0}\right)$ puede calcularse de la siguiente manera [99, 100, 101] (Ver también el trabajo [122]),

$$
\begin{aligned}
\frac{L^{\prime}\left(r_{0}\right)}{2} & =-\left.\frac{g(r)}{\sqrt{f^{2}(r)-f^{2}\left(r_{0}\right)}}\right|_{r \rightarrow r_{0}}+f^{\prime}\left(r_{0}\right) \int_{r_{0}}^{\infty} \frac{f(r) g(r)}{\left(f^{2}(r)-f^{2}\left(r_{0}\right)\right)^{\frac{3}{2}}} d r \\
& =-\left.\frac{g(r)}{\sqrt{f^{2}(r)-f^{2}\left(r_{0}\right)}}\right|_{r \rightarrow r_{0}}+f^{\prime}\left(r_{0}\right) \int_{r_{0}}^{\infty} d r \frac{g(r)}{f^{\prime}(r)} \frac{d}{d r}\left(-\frac{1}{\sqrt{f^{2}(r)-f^{2}\left(r_{0}\right)}}\right) \\
& =-\left.\frac{f^{\prime}\left(r_{0}\right) g(r)}{f^{\prime}(r) \sqrt{f^{2}(r)-f^{2}\left(r_{0}\right)}}\right|_{r \rightarrow \infty} ^{\infty}+\int_{r_{0}}^{\infty} d r \frac{f^{\prime}\left(r_{0}\right)}{\sqrt{f^{2}(r)-f^{2}\left(r_{0}\right)}} \frac{d}{d r}\left(\frac{g(r)}{f^{\prime}(r)}\right),
\end{aligned}
$$

donde se ha integrado por partes al pasar de la segunda a la tercera linea. Como el primer término del lado derecho de la tercera linea es nulo en todos los fondos de gravedad estudiados en este capítulo se tiene

$$
L^{\prime}\left(r_{0}\right)=2 \int_{r_{0}}^{\infty} d r \frac{f^{\prime}\left(r_{0}\right)}{\sqrt{f^{2}(r)-f^{2}\left(r_{0}\right)}} \frac{d}{d r}\left(\frac{g(r)}{f^{\prime}(r)}\right) .
$$

La energía de la configuración $q \bar{q}$ fue propuesta en $[45,46]$ como la longitud de la solución de cuerda (3.6) en la métrica efectiva 2-dimensional (3.4),

$$
E=\frac{1}{2 \pi \alpha^{\prime}} \int d \sigma \sqrt{f^{2}(r) \dot{x}(\sigma)^{2}+g^{2}(r) \dot{r}(\sigma)^{2}}
$$

Usando (3.6) se puede escribir la energía en el gauge $x$ y en el $r$ de la siguiente forma

\footnotetext{
${ }^{3}$ Para $f^{\prime}\left(r_{0}\right)=0$ hay una divergencia logarítmica dependiente de $r_{0}$ en (3.9) (en [122] hay una discusión general sobre divergencias en la función longitud).
} 


$$
\begin{aligned}
E\left(r_{0}\right) & =\frac{1}{2 \pi \alpha^{\prime}} \int_{-L / 2}^{L / 2} d x \frac{f^{2}(r(x))}{f\left(r_{0}\right)} \\
& =\frac{1}{\pi \alpha^{\prime}} \int_{r_{0}}^{\infty} d r \frac{g(r) f(r)}{\sqrt{f^{2}(r)-f^{2}\left(r_{0}\right)}} .
\end{aligned}
$$

La energía calculada con la expresión (3.11) diverge debido a la extensión infinita de la cuerda ${ }^{4}$. La interpretación de esta divergencia proviene de que la ecuación (3.11) involucra, además de la energía potencial entre los quarks, la autoenergía (masa) de los mismos [45, 46]. Con el fin de obtener una cantidad con sentido físico y encontrar el potencial entre quarks, debemos comparar (3.11) con respecto a un estado de referencia teniendo la precaución de sustraer una cantidad independiente de $r_{0}$. Es costumbre tomar la longitud de una cuerda recta a lo largo de la coordenada $r$ que sale de infinito y explora el interior del espacio-tiempo, con todas las otras coordenadas fijadas a constante, como la masa desnuda del quark. Nombrando $r=r_{\min }$ al valor mínimo permitido por la geometría (3.2) para la coordenada radial, sea por la existencia de un horizonte (por ejemplo, fondos de AdS en coordenadas de Poincaré o agujeros negros térmicos ), o porque realmente el espacio-tiempo se acaba suavente (ej. el Soliton AdS de Witten, la solución de Maldacena-Nuñez y la de Klebanov-Strassler), la masa del quark toma la forma ${ }^{5}$

$$
m_{q}=\frac{1}{2 \pi \alpha^{\prime}} \int_{r_{\text {min }}}^{\infty} g(r) d r
$$

La energía potencial entre el par quark-antiquark obtenida de (3.11) luego de sustraer la autoenergía de los mismos (3.14) es

$$
\begin{aligned}
E_{q \bar{q}}\left(r_{0}\right) & =E\left(r_{0}\right)-2 m_{q} \\
& =\frac{1}{\pi \alpha^{\prime}}\left[\int_{r_{0}}^{\infty} \frac{g(r) f(r)}{\sqrt{f^{2}(r)-f^{2}\left(r_{0}\right)}} d r-\int_{r_{\text {min }}}^{\infty} g(r) d r\right] .
\end{aligned}
$$

Eliminando $r_{0}$ de (3.9) y (3.15) se obtiene la propuesta que nos ofrece la correspondencia AdS/CFT para la energía potencial entre quarks, $V_{\text {string }}(L)$, en el límite de grandes valores de la constante de 't Hooft. En las secciones que siguen se graficarán estas relaciones en varios ejemplos y se analizará su forma funcional. Por completitud, la derivada de (3.15) es

$$
E_{q \bar{q}}^{\prime}\left(r_{0}\right)=\frac{1}{\pi \alpha^{\prime}}\left[-\left.\frac{g(r) f(r)}{\sqrt{f^{2}(r)-f^{2}\left(r_{0}\right)}}\right|_{r=r_{0}}+\int_{r_{0}}^{\infty} d r \frac{f(r) g(r) f\left(r_{0}\right) f^{\prime}\left(r_{0}\right)}{\left(f^{2}(r)-f^{2}\left(r_{0}\right)\right)^{\frac{3}{2}}}\right] .
$$

\footnotetext{
${ }^{4}$ En general, la divergencia en (3.11) es independiente de $r_{0}$. Adicionalmente puede aparecer una divergencia que sí depende de $r_{0}$ en las ecuaciones (3.9) y (3.11) cuando la cuerda explora regiones en donde $f^{\prime}\left(r_{0}\right)=0$.

${ }^{5}$ Véase la discusión al final de las secciones 3.2.1 y 3.2.3 para aspectos relacionados con la interpretación del estado de referencia en fondos gravitatorios suaves.
} 
Usando la primera linea de (3.10) se obtiene [99, 100, 101]

$$
E_{q \bar{q}}^{\prime}\left(r_{0}\right)=\frac{1}{2 \pi \alpha^{\prime}} f\left(r_{0}\right) L^{\prime}\left(r_{0}\right) \Rightarrow \frac{d E_{q \bar{q}}}{d L}=\frac{1}{2 \pi \alpha^{\prime}} f\left(r_{0}\right),
$$

donde $r_{0}$ en la última expresión debe entenderse como la función $r_{0}(L)$ obtenida de invertir (3.9).

Voy a finalizar esta sección describiendo algunas condiciones que debe satisfacer cualquier potencial que pretenda describir la interacción entre quarks. Las llamadas condiciones de " concavidad" demostradas en [112] son

$$
\frac{d V}{d L}>0, \quad \frac{d^{2} V}{d L^{2}} \leq 0
$$

Estas condiciones son válidas independientemente del grupo de gauge y los detalles del sector de materia. La interpretación física de (3.17) nos dice que la fuerza entre el par quark-antiquark es:(i) siempre atractiva y (ii) una función no creciente de su distancia de separación. De (3.9),(3.15),(3.16) se encuentra que para la función propuesta por la conjetura $V_{\text {string }}(L)$ se tiene [109]

$$
\frac{d V_{\text {string }}}{d L}=\frac{d E_{q \bar{q}}}{d r_{0}} \frac{d r_{0}}{d L}=\frac{1}{2 \pi \alpha^{\prime}} f\left(r_{0}\right), \quad \frac{d^{2} V_{\text {string }}}{d L^{2}}=\frac{1}{2 \pi \alpha^{\prime}}\left(\frac{d L}{d r_{0}}\right)^{-1} f^{\prime}\left(r_{0}\right) .
$$

La primera condición siempre se satisface en fondos de gravedad duales, debido a que por definición $f(r)>0$. Aunque en cada uno de los ejemplos estudiados en esta tesis se tiene $f^{\prime}(r)>0$, la segunda condición no se va a satisfacer cuando $L^{\prime}\left(r_{0}\right)$ sea positiva. Voy a presentar casos en donde aparecen estas manifestaciones no física y mostraré que en estas circunstancias la solución para la forma de embeber la cuerda (3.7)-(3.9) es inestable ante pequeñas perturbaciones. Esta última afirmación es el motivo del presente capítulo.

\subsubsection{Análisis de Estabilidad de embeddings de cuerdas clásicas}

Se estudiará en esta sección la estabilidad de la solución clásica $\left(r_{\mathrm{cl}}(\sigma), x_{\mathrm{cl}}(\sigma)\right)$ dada por (3.7)(3.9) ante pequeñas (y lineales) perturbaciones. Una fluctuación general alrededor de la solución puede escribirse como

$$
X^{\mu}=\left(\tau, x_{\mathrm{cl}}(\sigma)+\delta x_{1}(\tau, \sigma), \delta x_{2}(\tau, \sigma), \delta x_{3}(\tau, \sigma), r_{\mathrm{cl}}(\sigma)+\delta r(\tau, \sigma), \theta^{a}+\delta \theta^{a}(\tau, \sigma)\right) .
$$

Es posible usar la invarianza ante difeomorfismos de la acción para fijar $t=\tau$ y olvidarnos de la ecuación de movimiento en la dirección $t$. Para las clases de métricas consideradas en (3.2), las fluctuaciones $\delta x_{2}$ y $\delta x_{3}$ se desacoplan y satisfacen, como era de esperar, la misma ecuación de movimiento, las fluctuaciones en los ángulos $\delta \theta^{a}$ se mezclan entre ellas (en el caso general de variedades compactas) y dan lugar a 5 ecuaciones de movimiento (no voy a analizar fluctuaciones angulares en el presente trabajo y pueden fijarse a 0 de forma consistente). Finalmente las fluctuaciones $\delta x_{1}$ y $\delta r$ se mezclan dando lugar a 2 ecuaciones acopladas. 
Es fácil de ver que las ecuaciones de movimiento obtenidas en el gauge $r$ son proporcionales a las obtenidas en el gauge $x$. El difeomorfismo remanente debe ser usado para fijar la orientación del vector $\left(\delta r, \delta x_{1}\right)$ (fluctuaciones en el plano) en cada punto de la solución $\left(r_{\mathrm{cl}}(\sigma), x_{\mathrm{cl}}(\sigma)\right)$. Después de imponer un vínculo de gauge una ecuación describe las fluctuaciones en el plano $\left(r, x_{1}\right)$ y tenemos un sistema de ecuaciones diferenciales bien definido. La elección física del gauge (n-gauge) es el que orienta la fluctuación a lo largo de la dirección normal a $\left(r_{\mathrm{cl}}(\sigma), x_{\mathrm{cl}}(\sigma)\right)$, en general esto significa que se están teniendo en cuenta ambas fluctuaciones, en la coordenada $r$ y en la $x_{1}$.

Hay otras dos posibilidades naturales consideradas en la literatura correspondientes a fijar $\delta x_{1}(\tau, \sigma)=0$ (x-gauge) o $\delta r(\tau, \sigma)=0$ ( $r$-gauge). Los fijados de gauge $n$ y $x$, como se ha mencionado en la sección previa, parametrizan las fluctuaciones a lo largo de toda la solución clásica pero las ecuaciones de movimiento resultantes son tediosas debido a que las fluctuaciones $\delta r$ dan contribuciones adicionales provenientes de fluctuaciones de la métrica [99, 100, 101, 105].

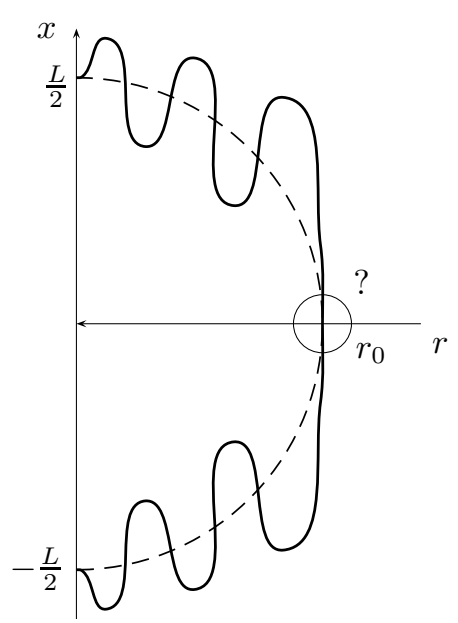

Figura 3.1: r-gauge: La línea discontinua representa la forma clásica de embeber la cuerda que uno perturba. En el tip $r_{0}$, la fluctuación esta orientada a lo largo de la cuerda, y por lo tanto no es física.

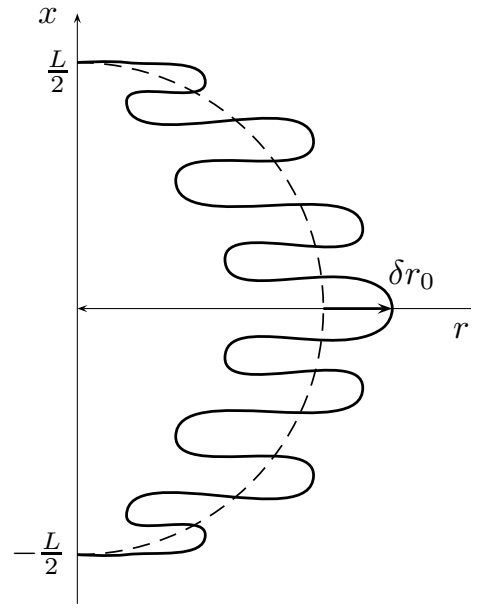

Figura 3.2: $x$-gauge: La línea discontinua representa la forma clásica de embeber la cuerda que uno perturba. Esta es una elección con sentido físico bien definido en toda la solución, las fluctuaciones gráficadas (pares) cambian la posición del tip.

Nótese que a primera vista los fijados de gauge $n$ y $x$ aparentemente permiten oscilaciones del tip, mientras que el fijado de gauge $r$ no lo permite. Voy a elegir trabajar en el gauge $r$, lo que significa tener $\delta r(\tau, \sigma)=0$ y trabajar con ecuaciones de movimiento más simples definidas en la mitad de la solución. Con el fin de obtener soluciones con sentido será entonces necesario analizar las condiciones de borde en el tip $\left(r_{0}, 0\right)$ del embedding. Además, como se están considerando fluctuaciones a lo largo de la coordenada $x_{1}$, precisamente en el tip la fluctuación $\delta x_{1}$ se orienta a lo largo del volumen de mundo de la cuerda y no transversalmente al mismo, es decir: la fluctuación 
$\delta x_{1}$ en el gauge $r$ no es física en el tip del embedding. El análisis requerido ha sido desarrollado en [98],[99, 100, 101] y será discutido en secciones posteriores. Por último, otra ventaja de usar el gauge $r$ es que nos quedan expresiones cerradas de las ecuaciones de movimiento linealizadas para las fluctuaciones (ver (3.22)-(3.23)), la isometría a lo largo de $x_{1}$ implica que no se necesita la solución analítica explicita de $x_{c l}(r)$, lo que contribuye a las ecuaciones de movimiento es su derivada (3.7) (como ejemplo comparar (A.10) con (A.11)). Me gustaría hacer referencia al trabajo [98] en donde se consideraron fluctuaciones $\delta r$ en el gauge $\delta x_{1}=0$ sobre la mitad de la solución clásica parametrizada en el gauge $r$ (ver debajo ecuaciones $(3.27),(3.28)$ ).

En lo que sigue voy estudiar las ecuaciones de movimiento para las fluctuaciones $\delta x_{i}$, estas se obtienen usando el ansatz

$$
t=\tau, \quad x_{1}=x_{\mathrm{cl}}(r)+\delta x_{1}(t, r), \quad x_{2}=\delta x_{2}(t, r), \quad x_{3}=\delta x_{3}(t, r), \quad r=\sigma,
$$

en la acción (3.1). Expandiendo a segundo orden en fluctuaciones se obtiene

$$
\begin{aligned}
2 \pi \alpha^{\prime} \mathcal{L}^{(2)}= & \frac{1}{g(r) f(r) \sqrt{f^{2}(r)-f^{2}\left(r_{0}\right)}}\left[h^{2}(r)\left(f^{2}(r)-f^{2}\left(r_{0}\right)\right)\left(\delta \dot{x}_{1}\right)^{2}-\left(f^{2}(r)-f^{2}\left(r_{0}\right)\right)^{2}\left(\delta \dot{x}_{1}\right)^{2}\right. \\
& \left.+f^{2}(r) h^{2}(r)\left(\left(\delta \dot{x}_{2}\right)^{2}+\left(\delta \dot{x}_{3}\right)^{2}\right)-f^{2}(r)\left(f^{2}(r)-f^{2}\left(r_{0}\right)\right)\left(\left(\delta \dot{x}_{2}\right)^{2}+\left(\delta \dot{x}_{3}\right)^{2}\right)\right], \quad(3.21)
\end{aligned}
$$

donde $h^{2}(r)=g_{x}(r) g_{r}(r)$. La ecuación de Euler-Lagrange para la fluctuación $\delta x_{1}$ es

$$
\left[\frac{d}{d r}\left(\frac{\left(f^{2}(r)-f^{2}\left(r_{0}\right)\right)^{\frac{3}{2}}}{g(r) f(r)} \frac{d}{d r}\right)+\omega^{2} \frac{h^{2}(r) \sqrt{f^{2}(r)-f^{2}\left(r_{0}\right)}}{g(r) f(r)}\right] \delta x_{1}(r)=0,
$$

en donde se ha factorizado la dependencia temporal de las fluctuaciones como $\delta x(t, r)=\delta x(r) e^{-i \omega t}$. Las ecuaciones para las fluctuaciones transversales al plano $\left(r, x_{1}\right)$ obtenidas de (3.21) son

$$
\left[\frac{d}{d r}\left(\frac{f(r) \sqrt{f^{2}(r)-f^{2}\left(r_{0}\right)}}{g(r)} \frac{d}{d r}\right)+\omega^{2} \frac{h^{2}(r) f(r)}{g(r) \sqrt{f^{2}(r)-f^{2}\left(r_{0}\right)}}\right] \delta x_{m}(r)=0, \quad m=2,3 .
$$

Las ecuaciones (3.22)-(3.23) son ecuaciones diferenciales del tipo Sturm-Liouville definidas en la semi-recta $r_{\min } \leq r_{0} \leq r<\infty$ y estoy interesado en analizar la existencia de inestabilidades, en particular, determinando el rango de valores de $r_{0}$ para el cual $\omega^{2}<0$.

Las condiciones de borde a imponer en el problema son del tipo Dirichlet, esto se traduce en fluctuaciones que dejan los extremos de la cuerda fijos en el borde $\left.\delta x_{i}(\tau, \sigma)\right|_{r=\infty}=0$. Pero, como el gauge $r$ parametriza solo la mitad de $\left(r_{\mathrm{cl}}(\sigma), x_{\mathrm{cl}}(\sigma)\right)$ se requiere un estudio adicional de las condiciones de borde en el tip $r=r_{0}$ (punto singular de (3.22)-(3.23)). Voy a comenzar analizando (3.23), la expansión alrededor del tip nos $\mathrm{da}^{6}$

$\frac{d}{d r}\left(\sqrt{r-r_{0}} \frac{d \delta x_{m}(r)}{d r}\right)+\frac{\omega^{2} h^{2}\left(r_{0}\right)}{2 f\left(r_{0}\right) f^{\prime}\left(r_{0}\right)} \frac{1}{\sqrt{r-r_{0}}} \delta x_{m}(r) \approx 0 \Longrightarrow \delta x_{m}(r) \approx C_{0}+C_{1} \sqrt{r-r_{0}}+O\left(r-r_{0}\right)$.

\footnotetext{
${ }^{6}$ Considero a $f(r)$ como una función creciente de $r$ que no tiene ceros excepto, quizá, en el fondo de la geometría $r=r_{\min }$.
} 
Aquí $C_{0,1}$ son constantes arbitrarias correspondientes a las dos soluciones independientes de la ecuación diferencial (3.23), las cuales una vez elegidas, determinan en su totalidad la expansión en serie para $\delta x_{m}(r)$. Físicamente estas se corresponden con fluctuaciones pares e impares alrededor del tip respectivamente (una vez que las pegamos con las fluctuaciones alrededor de la otra mitad de la solución, las cuales obviamente satisfacen las ecuaciones de movimiento). Un conjunto discreto de valores $\omega^{2}$ es esperable si existen soluciones no normalizables en el límite de $r$ grande, también se espera que la solución par tenga el menor autovalor $\omega^{2}$ (ver apéndice A.2).

Ahora analizemos las fluctuaciones sobre el plano $\delta x_{1}$. Expandiendo (3.22) alrededor de $r=r_{0}$ se encuentra

$\frac{d}{d r}\left(\left(r-r_{0}\right)^{\frac{3}{2}} \frac{d \delta x_{1}(r)}{d r}\right)+\frac{\omega^{2} h^{2}\left(r_{0}\right)}{2 f\left(r_{0}\right) f^{\prime}\left(r_{0}\right)} \sqrt{r-r_{0}} \delta x_{1}(r) \approx 0 \Longrightarrow \delta x_{1}(r) \approx C_{0}^{\prime}+C_{1}^{\prime} \frac{1}{\sqrt{r-r_{0}}}+O\left(\sqrt{r-r_{0}}\right)$.

Aparece un desarrollo singular para $\delta x_{1}$ en el tip y uno esta tentado a cancelarlo imponiendo $C_{1}^{\prime}=0$. Sin embargo, como se mencionó anteriormente se debe tener en cuenta que el gauge $r$ implica que, en el tip, $\delta x_{1}$ esta dirigido a lo largo del volumen de mundo de la cuerda y no transversal a él, entonces, en el gauge $r$ el desplazamiento $\delta x_{1}$ (en el tip) no es físico. Con motivo de dar una interpretación física a las constantes $C_{0,1}^{\prime}$ en (3.25) voy a cambiarme del gauge $r$ al gauge $x$ (confrontar con [96],[99, 100, 101]). El cambio de gauge en el ansatz (3.20) puede implementarse mediante un cambio de variables sobre la solución (3.22) desde $r$ a una nueva variable que llamaré $u$. Esto puede ser implementado perturbativamente y a primer orden en las fluctuaciones la relación es $[99,100,101]$,

$$
u=r+\Delta(t, r) \quad \text { donde } \quad \Delta(t, r)=\frac{\delta x_{1}(t, r)}{x_{\mathrm{cl}}^{\prime}(r)} .
$$

Esta transformación nos permite realizar el cambio de gauge deseado ya que

$$
\begin{aligned}
x_{1} & =x_{\mathrm{cl}}(r)+\delta x_{1}(t, r)=x_{\mathrm{cl}}(u-\Delta(t, r))+\delta x_{1}(t, r) \\
& \approx x_{\mathrm{cl}}(u)-x_{\mathrm{cl}}^{\prime}(r) \frac{\delta x_{1}(t, r)}{x_{\mathrm{cl}}^{\prime}(r)}+\delta x_{1}(t, r)=x_{\mathrm{cl}}(u) \\
r & \approx u-\frac{\delta x_{1}(t, u)}{x_{\mathrm{cl}}^{\prime}(u)}
\end{aligned}
$$

aquí $r_{0} \leq u<\infty$. Este es precisamente el gauge empleado en [98]. El segundo término en el lado derecho de (3.28) es interpretado como la fluctuación en la dirección $r$ inducida por la (gauge $r$ ) fluctuación en $x_{1}$. Ahora es fácil de ver que es finita. El desarrollo asintótico (3.25) y la expansión alrededor del tip de (3.7), $x_{\mathrm{cl}}^{\prime}(r) \sim\left(r-r_{0}\right)^{-1 / 2}$, insertada en (3.28) es

$$
r \approx r_{0}-\alpha\left(C_{0}^{\prime} \sqrt{u-r_{0}}+C_{1}^{\prime}\right)+O\left(u-r_{0}\right),
$$

con $\alpha$ una constante finita. El resultado (3.29) muestra que la fluctuación física $\delta r$ originada por la 
fluctuación no física $\delta x_{1}$ en el tip es manifiestamente finita. Entonces podemos interpretar a $\left(C_{0}^{\prime}\right)$ $C_{1}^{\prime}$ en (3.25) como las fluctuaciones que (no) mueven la posición del tip.

En las secciones siguientes voy a estudiar soluciones numéricas de (3.22) para varios fondos gravitatorios determinando los autovalores más bajos que dan soluciones normalizables. Voy a resolver (3.22) mediante el método de shooting integrando numéricamente desde $r_{0}$ hasta un valor grande $r_{\infty}$. Los valores permitidos para $\omega^{2}$ se obtendrán de imponer que la solución numérica se anule en $r=r_{\infty}$. Las condiciones de borde en el tip correspondientes a soluciones pares son $C_{0}^{\prime}=0$ y $C_{1}^{\prime}$ arbitrario, por propósitos numéricos se utiliza $C_{1}^{\prime}=1$, este valor fija la normalización de la fluctuación. Una solución par alrededor del tip satisface

$$
\left.\frac{d \delta r(t, r)}{d x_{1}}\right|_{r=r_{0}}=0 \quad \text { where } \quad \delta r(t, r)=-\frac{\delta x_{1}(t, r)}{x_{\mathrm{cl}}^{\prime}(r)} .
$$

Usando (3.27) se puede escribir (3.30) en términos de $\delta x_{1}(r)$. Las condiciones de borde para soluciones pares de (3.22) se implementan numéricamente como

$$
\begin{aligned}
\delta x_{1}(r)+2\left(r-r_{0}\right) \frac{d \delta x_{1}(r)}{d r} & =0, & & r \rightarrow r_{0} \\
\sqrt{r-r_{0}} \delta x_{1}(r) & =1, & r & \rightarrow r_{0} .
\end{aligned}
$$

Para soluciones impares $C_{0}^{\prime}=1$ y $C_{1}^{\prime}=0$ se implementan como

$$
\begin{array}{rlrl}
\delta x_{1}(r)+2\left(r-r_{0}\right) \frac{d \delta x_{1}(r)}{d r} & =1, & & r \rightarrow r_{0} \\
\sqrt{r-r_{0}} \delta x_{1}(r)=0, & r & \rightarrow r_{0} .
\end{array}
$$

Resumiendo, en fondos gravitatorios generales, la relación funcional de la solución clásica (3.7) entre las coordenadas $x_{1}$ y $r$ en el tip toman la forma $x_{\mathrm{cl}}^{2}(r) \approx r-r_{0}$, y el desarrollo asintótico de las fluctuaciones en $x_{1}$, en el gauge $\mathrm{r}$, es de la forma (3.25). Aunque aparezca una parte divergente en (3.25), un cambio de gauge apropiado muestra que las partes divergentes y no divergentes corresponden respectivamente a fluctuaciones (física) pares e impares alrededor del tip.

\subsection{Fondos Gravitatorios}

En esta sección voy a calcular los embeddings de cuerdas (3.6) duales a lazos de Wilson rectangulares para un número de casos paradigmáticos. Se resumirán los casos de $\operatorname{Ad} S_{5} \times S^{5}[45,46]$ y $A d S_{5}$-Schwarzschild $\times S^{5}$ [102]. Luego, se realizará el análisis numérico de las ecuaciones (3.9)

y (3.15) para los fondos de Maldacena-Nuñez [6], Klebanov-Strassler [7] y la generalización de Maldacena-Nuñez $[114,115]$. En todos los caso la geometría es soportada por $p$-flujos no triviales, pero estos no son relevantes en los cálculos. 


\subsection{1. $A d S_{5} \times S^{5}$}

Esta solución de gravedad es dual a $\mathcal{N}=4$ SYM con grupo de gauge $G=S U(N)$ en la fase de Coulomb. La curvatura de AdS $R$ se relaciona con la teoría de gauge mediante la constante de 't Hooft $\lambda$ como $R^{4}=\alpha^{\prime 2} \lambda$ y el flujo de la 5 -forma que soporta la geometría $N=\int_{S^{5}} F_{5}$ se relaciona con el rango del grupo de gauge a través de $N=\operatorname{Rank}(G)[1,4,5]$. La invarianza conforme de la teoría de gauge implica un desarrollo tipo Coulomb para el potencial entre quarks $V(L) \sim 1 / L$. Lo novedoso del cálculo en el lado gravitatorio es que permite obtener el coeficiente de proporcionalidad y su dependencia en el acoplamiento de gauge.

\section{Coordenadas de Poincaré $[45,46]$}

Este sistema de coordenadas describe la teoría de gauge formulada en $\mathbb{R}^{3,1}$. La métrica se escribe como (compárese con la coordenadas utilizadas en la sección 1.1.3 en donde $z=\frac{1}{r}$ )

$$
d s^{2}=\frac{r^{2}}{R^{2}}\left(-d t^{2}+d x_{i} d x_{i}\right)+R^{2} \frac{d r^{2}}{r^{2}}+R^{2} d \Omega_{5}^{2} .
$$

Se encuentra que $f^{2}(r)=r^{4} / R^{4}, g^{2}(r)=1$. El rango de la coordenada radial es $0<r<\infty$, en $r=0$ hay un horizonte de Killing. La ecuación (3.7) puede ser resuelta analíticamente en el gauge $r$, se obtiene

$$
x_{\mathrm{cl}}(r)= \pm\left\{c t e-\frac{R^{2}}{4 r_{0}} \mathrm{~B}\left(\left(\frac{r_{0}}{r}\right)^{4} ; \frac{3}{4}, \frac{1}{2}\right)\right\}, \quad r_{0} \leq r<\infty
$$

donde $\mathrm{B}(z ; a, b)$ is la función beta incompleta $\mathrm{B}(z ; a, b)=\int_{0}^{z} t^{a-1}(1-t)^{b-1} d t$. Las condiciones de borde fijan la constante en (3.34) y relaciona los parámetros $r_{0}$ y $L$, fijando $x_{\mathrm{cl}}\left(r_{0}\right)=0 \mathrm{y}$ $x_{\mathrm{cl}}(\infty)= \pm L / 2$ se obtiene $[45,46]$,

$$
L\left(r_{0}\right)=\frac{R^{2}}{2 r_{0}} \mathrm{~B}\left(\frac{3}{4}, \frac{1}{2}\right)=\frac{R^{2}}{r_{0}} \frac{(2 \pi)^{\frac{3}{2}}}{\Gamma\left[\frac{1}{4}\right]^{2}} .
$$

La energía (3.15) toma la forma $[45,46]$

$$
E_{q \bar{q}}\left(r_{0}\right)=\frac{r_{0}}{\pi \alpha^{\prime}}(K(-1)-E(-1))=-\frac{r_{0}}{2 \pi \alpha^{\prime}} \frac{(2 \pi)^{\frac{3}{2}}}{\Gamma\left[\frac{1}{4}\right]^{2}},
$$

aquí, $K(m), E(m)$ son las integrales elípticas completas de primer y segundo tipo respectivamente. Eliminando $r_{0}$ de las expresiones (3.35)-(3.36) la propuesta de la conjetura AdS/CFT para la energía de interacción entre quarks en la representación fundamental del grupo de gauge en el límite $\lambda$ grande para la teoría $\mathcal{N}=4 \mathrm{SYM}$ es $[45,46]$

$$
V_{\text {string }}(L)=-\frac{(2 \pi)^{2}}{\Gamma\left[\frac{1}{4}\right]^{4}} \frac{R^{2} / \alpha^{\prime}}{L} \sim-\frac{\sqrt{\lambda}}{L} .
$$

Como es de esperar debido a la invarianza conforme se obtuvo un potencial de Coulomb atractivo. El resultado importante es la dependencia en $\sqrt{\lambda}=\left(g_{Y M}^{2} N\right)^{\frac{1}{2}}$ (recordar que en el régimen 
perturbativo el factor de proporcionalidad es $\lambda$ ). Esto sugiere que en el regimen de acoplamiento fuerte existe algún tipo de renormalización de las cargas $[45,46]$. Nótese que para obtener la cantidad negativa (3.37) habiendo comenzado de una cantidad definida positiva (ver ec.(3.11)) fue crucial sustraer la masa de los quarks (3.14).

\section{Coordenadas Globales}

Este ejemplo va a ser discutido debido a que clarifica conceptos involucrados en el proceso de sustracción de la masa (3.15) en duales gravitatorios suaves y completos (ver secciones 3.2.3, 3.2.4, $3.2 .5)$.

Cálculos realizados en coordenadas globales representan una teoría de gauge $\mathcal{N}=4$ SYM definida sobre $S^{3} \times \mathbb{R}$. La métrica de AdS se escribe

$$
d s^{2}=R^{2}\left[-\cosh ^{2} \rho d t^{2}+d \rho^{2}+\sinh ^{2} \rho d \Omega_{3}^{2}\right] .
$$

Todas las coordenadas son adimensionales, con el radio de AdS, $R$, fijando la escala. Se escribe la métrica de $S^{3}$ como $d \Omega_{3}^{2}=d \theta_{1}^{2}+\sin ^{2} \theta_{1}\left(d \theta_{2}^{2}+\sin ^{2} \theta_{2} d \varphi^{2}\right)$. Siendo $\varphi$ una coordenada cíclica, el ansatz apropiado para la cuerda es $t=\tau, \rho=\rho(\sigma), \varphi=\sigma$, esto conduce a $f^{2}(\rho)=\frac{1}{4} \sinh ^{2} 2 \rho, g^{2}(\rho)=$ $\cosh ^{2} \rho$. Las coordenadas angulares remanentes deben ser fijadas a $\theta_{i}=\frac{\pi}{2}$ (el ecuador de $S^{3}$ ) en orden de satisfacer las ecuaciones de movimiento. La carga conservada debida a la coordenada cíclica $\varphi$ conduce a una ecuación de movimiento unidimensional de energía cero

$$
\dot{\rho}^{2}+U(\rho)=0
$$

donde $\dot{\rho}=d \rho / d \varphi$ y el potencial $U(\rho)=\sinh ^{2} \rho\left(1-\frac{\sinh ^{2} 2 \rho}{\sinh ^{2} 2 \rho_{0}}\right) \cdot \rho_{0}$ es la posición radial mínima alcanzada por la cuerda cuando los extremos de la cuerda en infinito se separan en $\Delta \varphi=\Phi$. La relación (3.9) para $\Phi\left(\rho_{0}\right)$ se calcula trivialmente

$$
\Phi\left(\rho_{0}\right)=2 \int_{r_{0}}^{\infty} \frac{\sinh 2 \rho_{0}}{\sinh \rho \sqrt{\sinh ^{2} 2 \rho-\sinh ^{2} 2 \rho_{0}}} d \rho .
$$

Debido a que la teoría de gauge esta definida sobre $S^{3}$ existe una separación máxima para los quarks la cual corresponde a ubicarlos en las antípodas sobre el ecuador de la $S^{3}$. Esto resulta en una cuerda que alcanza el origen $\Phi(0)=\pi$ y que da lugar a una hoja de mundo recta y suave (extendida a lo largo de la coordenada $r$ ) parametrizada por dos mitades en $\varphi=\varphi_{0}$ y $\varphi=\pi+\varphi_{0}$. La energía (divergente) (3.13) de la configuración (3.39) es

$$
E\left(\rho_{0}\right)=\frac{R}{2 \pi \alpha^{\prime}} \int_{\rho_{0}}^{\infty} \frac{\sinh ^{2} 2 \rho}{\sinh \rho \sqrt{\sinh ^{2} 2 \rho-\sinh ^{2} 2 \rho_{0}}} d \rho .
$$

Substrayendo la masa de los quarks (3.15) se obtiene,

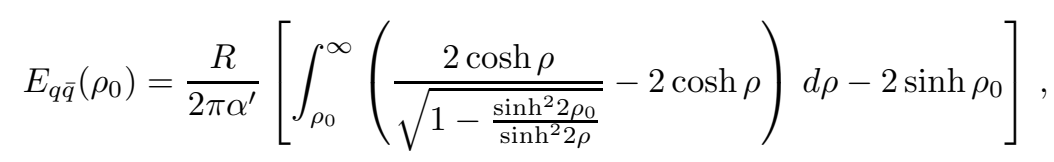


la cual es finita y definida negativa ( ver figura 3.3). El resultado finito (3.42) debe entenderse como resultante de comparar (3.41) con respecto al estado de referencia proveniente de la configuración de la cuerda recta con sus extremos en infinito y en las antípodas del ecuador de la $S^{3}$. Se interpreta esta última configuración como la única correspondiente a quarks "infinitamente"separados en la esfera. Nótese que el estado de referencia y la configuración que se esta analizando satisfacen diferentes condiciones de borde.

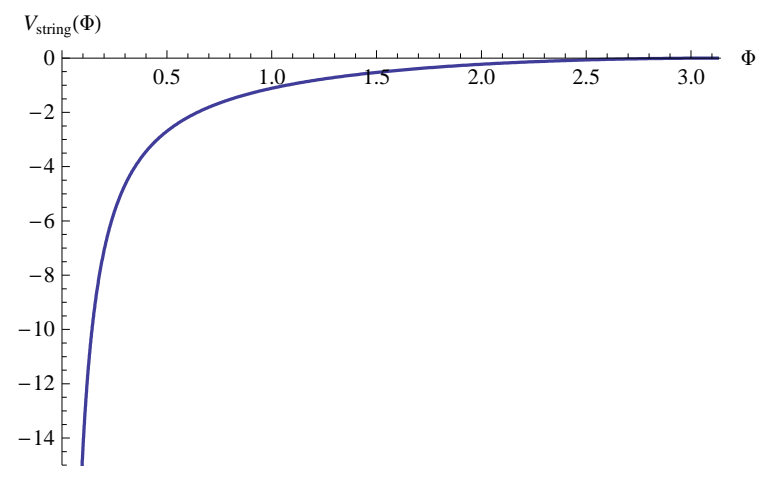

Figura 3.3: $V_{\text {string }}(\Phi)$ obtenido a partir de (3.40) y (3.42). Para ángulos de separación pequeños entre los extremos de la cuerda $\Phi \ll 1$ se encuentra el comportamiento de Coulomb esperable $V \sim \sqrt{\lambda} /(R \Phi)$. Para separaciones grandes la solución se desvía debido a que $S^{3}$ es compacta.

\subsection{2. $A d S_{5}-$ Schwarzschild $\times S^{5}$}

Teorías de gauge a temperatura finita se describen considerando soluciones de agujero negro en duales gravitatorios [19]. La geometría de horizonte cercano de $N$ D3-branas no extremales (negras) se conjetura dual a $\mathcal{N}=4 \mathrm{SYM}$ a temperatura finita. Como se explica en [19], el fondo de agujero negro apropiado para describir la teoría de gauge sobre $\mathbb{R}^{3,1}$ involucra un límite de masa infinita delicado del agujero negro $A d S_{5}$-Schwarzschild resultante en, ${ }^{7}$

$$
d s^{2}=\frac{r^{2}}{R^{2}}\left[-\left(1-\frac{\mu^{4}}{r^{4}}\right) d t^{2}+d x_{i} d x_{i}\right]+\frac{R^{2}}{r^{2}} \frac{1}{1-\frac{\mu^{4}}{r^{4}}} d r^{2}+R^{2} d \Omega_{5}^{2} .
$$

El horizonte del agujero negro esta localizado en $r=\mu$ y su temperatura es $T=\frac{\mu}{R^{2}} \pi$. Es conveniente trabajar con coordenadas adimensionales, escaleando $r=\mu \rho, t=R^{2} / \mu \bar{t}$ y $x=R^{2} / \mu$ y se obtiene

$$
d s^{2}=R^{2}\left[-\left(\rho^{2}-\frac{1}{\rho^{2}}\right) d \vec{t}^{2}+\rho^{2} d y_{i} d y_{i}+\frac{1}{\rho^{2}-\frac{1}{\rho^{2}}} d \rho^{2}+d \Omega_{5}^{2}\right] .
$$

La escala de la teoría de gauge adimensional $\bar{t}, y_{i}$ en (3.44) esta fijada por $R^{2} / \mu$ y se tiene $f^{2}(\rho)=\rho^{4}-1, g^{2}(\rho)=1$ y $\rho=1$ para la posición del horizonte. Las expresiones para la longitud

\footnotetext{
${ }^{7}$ El límite proviene de que la métrica es dependiente de solo una escala, al contrario del caso de masa finita en donde hay dos parámetros: la temperatura (masa del agujero negro) y el radio de AdS. Esta última geometría exhibe una transición de fase (Hawking-Page), la cual fue interpretada como dual a la transición de fase confinante/deconfinante en $\mathcal{N}=4$ sobre $S^{3}[19]$.
} 


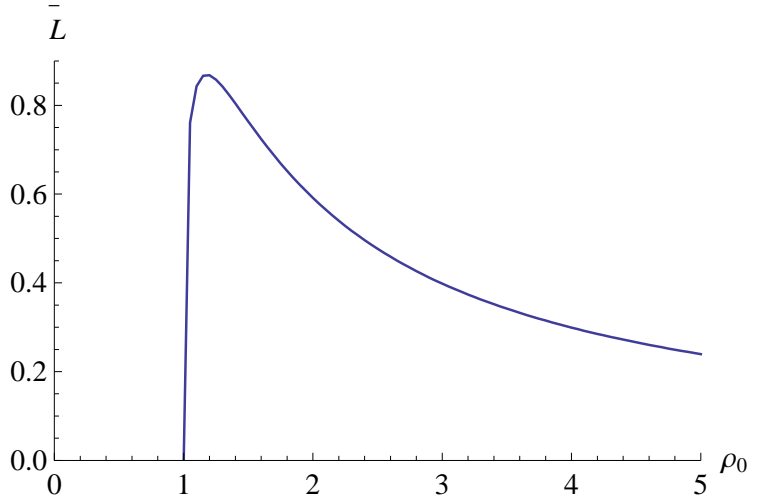

Figura 3.4: $L\left(\rho_{0}\right)$ (3.45): Se observa un máximo para $\rho_{0} \simeq 1,177$. Las dos ramas de la izquierda/derecha del máximo en $\rho_{0 c} \simeq 1,177$ dan lugar a una relación bivaluada para $V_{\text {string }}(L)$ en la fig. 3.5 .

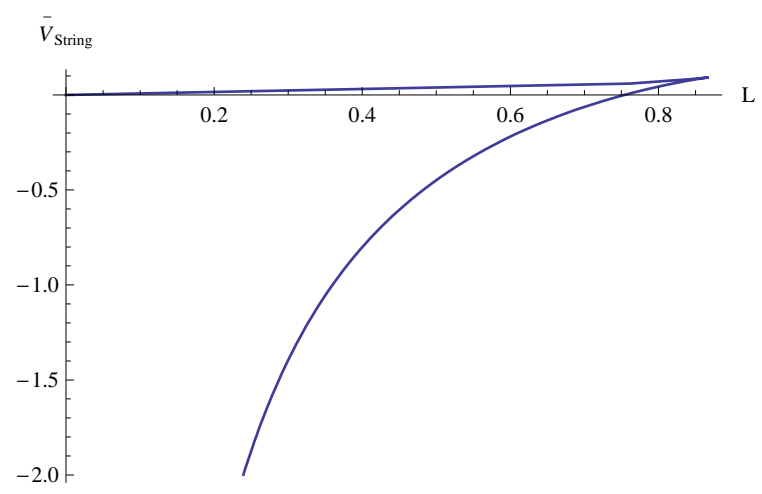

Figura 3.5: $V_{\text {string }}(L)$ bivaluada obtenida desde (3.45) y (3.46) eliminando numéricamente $\rho_{0}$. La curva superior correspondiente a la rama izquierda en la fig. 3.4 no satisface las condiciones $(3.17)$.

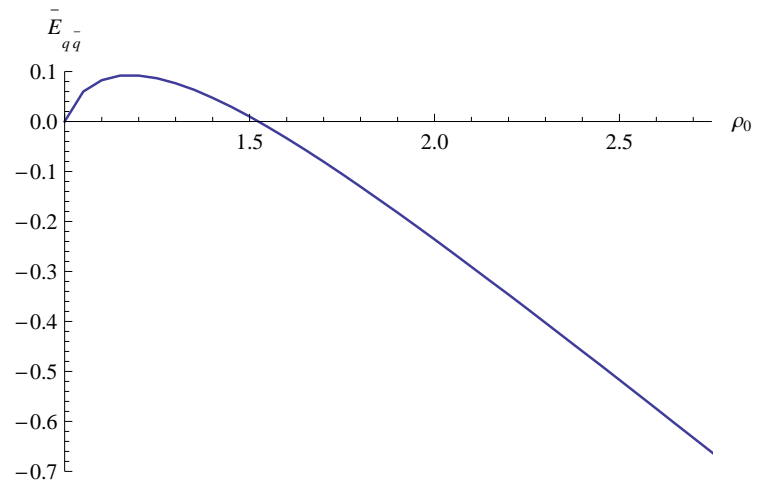

Figura 3.6: Función $\bar{E}_{q \bar{q}}\left(\rho_{0}\right)$ para $\mathcal{N}=4$ SYM térmico. La ecuación (3.16) garantiza la existencia de un extremo en $E_{q \bar{q}}\left(r_{0}\right)$ y $L\left(r_{0}\right)$ para el mismo valor de $r_{0}$. La curva corta el eje en $\rho_{0 m} \simeq 1,524$, para $\rho_{0}>\rho_{0 m}$ la solución en forma de U es un mínimo absoluto, para $\rho_{0}<\rho_{0 m}$ la solución de dos líneas rectas (referencia) es el mínimo absoluto.

de separación adimensional (3.9) y la energía potencial (3.15) para el par $q \bar{q}$ pueden calcularse analíticamente [99, 100, 101, 102, 103, 109]

$$
\begin{gathered}
\bar{L}\left(\rho_{0}\right)=\frac{(2 \pi)^{\frac{3}{2}}}{\Gamma\left[\frac{1}{4}\right]^{2}} \frac{\sqrt{\rho_{0}^{4}-1}}{\rho_{0}^{3}}{ }_{2} F_{1}\left(\frac{3}{4}, \frac{1}{2}, \frac{5}{4} ; \frac{1}{\rho_{0}^{4}}\right) \\
\bar{E}_{q \bar{q}}\left(\rho_{0}\right)=\frac{R^{2}}{\pi \alpha^{\prime}}\left[1-\frac{(2 \pi)^{\frac{3}{2}}}{2 \Gamma\left[\frac{1}{4}\right]^{2}} \rho_{0}{ }_{2} F_{1}\left(-\frac{1}{2},-\frac{1}{4}, \frac{1}{4} ; \frac{1}{\rho_{0}^{4}}\right)\right]
\end{gathered}
$$

Aquí $\rho_{0} \geq 1$ es la posición radial mínima alcanzada por la cuerda, el valor radial mínimo $r_{\min }$ en (3.15) ha sido tomado en la ubicación del horizonte $r_{\min }=\mu$. Se puede chequear fácilmente que en el límite de temperaturas bajas $L T \ll 1$ (correspondiente a $\rho_{0} \gg 1$ ) se recupera el comportamiento a temperatura cero (3.35)-(3.37). Se grafican en las figuras 3.4 y 3.6 la forma de la longitud (3.45) 
y la energía (3.46) como función de $\rho_{0}$. En la figura 3.5 se grafica la relación $V_{\text {string }}(L)$ obtenida a partir de (3.45)-(3.46) eliminando $\rho_{0}$, el resultado es una función bivaluada.

La figura 3.4 muestra un máximo $\bar{L}_{c} \simeq 0,869$ en $\rho_{0 c} \simeq 1,177$ lo cual implica que no existe solución suave que conecte al par de quarks para $\bar{L}>\bar{L}_{c}$. La única solución existente para $\bar{L}>$ $\bar{L}_{c}$ corresponde a dos cuerdas rectas que alcanzan el horizonte. Esta configuración, usada para la sustracción en (3.15), es interpretada como la correspondiente a un par de quarks libres. La existencia de un máximo en la función $L\left(r_{0}\right)$ para el fondo de agujero negro ha sugerido interpretarlo como el dual gravitatorio de un baño térmico de apantallamiento [102, 103]. La figura 3.4 también muestra la existencia de dos ramas de soluciones para cada distancia de separación $L<L_{c}$ entre los extremos de la cuerda. La rama izquierda $\left(L^{\prime}>0\right)$ da lugar a un potencial $V_{\text {string }}(L)$ que no satisface las condiciones (3.17) y por lo tanto no es física (curva superior en la figura 3.5). El resultado, como veremos, es que la teoría de cuerdas es sabia: la rama izquierda no debería ser válida debido a que es inestable ante pequeñas perturbaciones (ver sección 3.3.2).

El último punto para comentar es que aunque, como vimos, toda la región para la cual $L^{\prime}<0$ $\left(1,177<\rho_{0}<\infty\right)$ es estable ante pequeñas perturbaciones, se espera que la curva de abajo en la figura 3.5 sea metaestable cuando $E_{q \bar{q}}>0\left(0,754<\bar{L}<0,869\right.$ o $\left.1,177<\rho_{0}<1,524\right)$. La razón de esto es que el estado de referencia, las solución correspondiente a dos líneas rectas tiene $E_{q \bar{q}}=0 \mathrm{y}$ entonces es el mínimo absoluto en $0,754<\bar{L}<0,869$ (Ver fig. 3.6). Teniendo en cuentas estas dos últimas cosas en [102] se sugiere tomar $\bar{L}_{\max } \simeq 0,754$ como la longitud de apantallamiento.

\subsubsection{Maldacena-Núñez}

La región $r \approx 0$ de este fondo gravitatorio es dual al regimen IR de una teoría $\mathcal{N}=1 \mathrm{SYM}$ en $d=4$ más una torre de estados de Kaluza-Klein. La configuración de branas de prueba que permiten obtener esta solución consiste en $N$ D5-branas enroscadas sobre un 2-ciclo finito en el origen de un conifold resuelto. Cuando se tiene en cuenta la acción de las D5 sobre la geometría ocurre una transición (ver [117]) que nos conduce a un espacio-tiempo dotado de una $S^{2}$ que colapsa y una $S^{3}$ finita en el origen (como sucede en el conifold deformado, ver [118]). Esta solución fue encontrada independientemente en el contexto de supergravedades con simetrías de gauge (gauged sugra) en [120, 121].

La métrica se escribe [6]

$$
d s^{2}=\alpha^{\prime} N e^{\phi}\left[-d t^{2}+d x_{i} d x_{i}+d r^{2}+e^{2 h}\left(d \theta^{2}+\sin ^{2} \theta d \varphi^{2}\right)+\frac{1}{4}\left(w^{i}-A^{i}\right)^{2}\right],
$$

en donde $w^{i}(i=1,2,3)$ son las formas invariantes a derecha de $s u(2)$

$$
w^{1}+i w^{2}=e^{-i \psi}(d \tilde{\theta}+i \sin \tilde{\theta} d \tilde{\varphi}), \quad w^{3}=d \psi+\cos \tilde{\theta} d \tilde{\varphi}
$$


y $A^{i}, \phi, h$ esta dada por

$$
\begin{aligned}
A^{1} & =-a(r) d \theta, \quad A^{2}=a(r) \sin \theta d \varphi, \quad A^{3}=-\cos \theta d \varphi \\
a(r) & =\frac{2 r}{\sinh 2 r} \\
e^{2 h} & =r \operatorname{coth} 2 r-\frac{r^{2}}{\sinh ^{2} 2 r}-\frac{1}{4} \\
e^{2 \phi} & =e^{2 \phi_{0}} \frac{\sinh 2 r}{2 e^{h}} .
\end{aligned}
$$

$\phi_{0}$ es una constante de integración que fija el valor del dilatón $\phi$ en el origen (algunos autores escriben $\left.g_{s}=e^{\phi_{0}}\right)$.

Las coordenadas $t, x_{i}, r$ son adimensionales y su escala queda fijada por $\left(\alpha^{\prime} N\right)^{\frac{1}{2}}$. La métrica (3.47) es regular en el límite $r \rightarrow 0$ y la 2-esfera $(\theta, \varphi)$ colapsa suavemente, la topología del espaciotiempo resultante es de la forma $\mathcal{M}_{7} \times S^{3}$, contrariamente a los casos discutidos anteriormente en donde la topología tomaba la forma $\mathcal{M}_{5} \times X^{5}$ con $X^{5}$ compacto. La razón de esto es que la métrica de fondo (3.47) modela una teoría de gauge $5+1$ dimensional sobre la $D 5$-brana enroscada. Sin embargo, se espera tener una teoría efectiva $3+1$ dimensional a energías $E<1 / R_{S^{2}}$, donde $R_{S^{2}}$ es el radio de la esfera que envuelve la $D 5$.

El ansatz de la cuerda estática con forma de U nos conduce a $f^{2}(r)=g^{2}(r)=e^{2 \phi}$, con lo cual la longitud de separación (3.9) y la energía (3.15) toman la forma

$$
\begin{gathered}
\bar{L}\left(r_{0}\right)=2 \int_{r_{0}}^{\infty} \frac{e^{\phi\left(r_{0}\right)}}{\sqrt{e^{2 \phi(r)}-e^{2 \phi\left(r_{0}\right)}}} d r \\
\bar{E}_{q \bar{q}}\left(r_{0}\right)=\frac{N}{\pi}\left[\int_{r_{0}}^{\infty} \frac{e^{2 \phi(r)}}{\sqrt{e^{2 \phi(r)}-e^{2 \phi\left(r_{0}\right)}}} d r-\int_{0}^{\infty} e^{\phi(r)} d r\right] .
\end{gathered}
$$

La expresión para la energía puede ser reescrita de forma conveniente [91]

$$
\begin{aligned}
\bar{E}_{q \bar{q}}\left(r_{0}\right) & =\frac{N}{\pi}\left[\int_{r_{0}}^{\infty}\left(\frac{e^{2 \phi(r)}+e^{2 \phi\left(r_{0}\right)}-e^{2 \phi\left(r_{0}\right)}}{\sqrt{e^{2 \phi(r)}-e^{2 \phi\left(r_{0}\right)}}}-e^{\phi(r)}\right) d r-\int_{0}^{r_{0}} e^{\phi(r)} d r\right] \\
& =\frac{N}{\pi}\left[e^{\phi\left(r_{0}\right)} \frac{\bar{L}\left(r_{0}\right)}{2}+\int_{r_{0}}^{\infty} d r\left(\sqrt{e^{2 \phi(r)}-e^{2 \phi\left(r_{0}\right)}}-e^{\phi(r)}\right)-\int_{0}^{r_{0}} e^{\phi(r)} d r\right] .
\end{aligned}
$$

En el límite de $L$ grande la cuerda alcanza el fin del espacio $\left(r_{0} \rightarrow 0\right.$, ver figura 3.7) y los últimos dos términos en (3.52) no contribuyen (see [91]). En el límite de $\bar{L} \gg 1$ se obtiene, explicitando nuevamente las dimensiones [6]

$$
V_{\text {string }}(L) \approx \frac{e^{\phi_{0}}}{2 \pi \alpha^{\prime}} L \quad \Rightarrow \quad T_{\text {string }}=\frac{e^{\phi_{0}}}{2 \pi \alpha^{\prime}} .
$$

La métrica de fondo (3.47) predice, por lo tanto, confinamiento lineal para la separación entre quarks en perfecto acuerdo con las propiedades de su teoría de gauge dual $d=4, \mathcal{N}=1 \mathrm{SYM}$. A partir de la tensión de la cuerda (3.53) se puede ver que el valor del dilatón en el origen $\phi_{0}$ se 


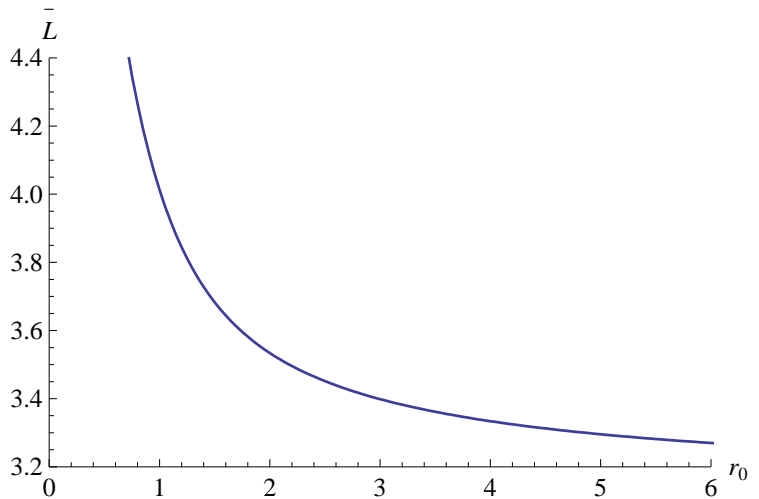

Figura 3.7: $\bar{L}\left(r_{0}\right)$ (3.50). Solución de MN.

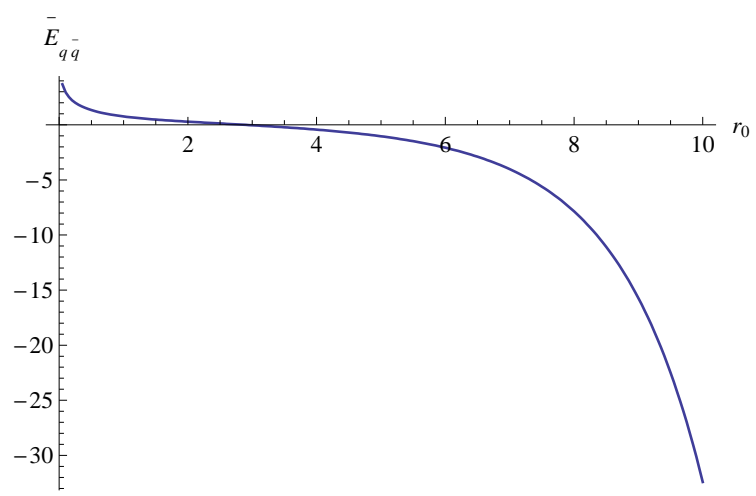

Figura 3.8: $E\left(r_{0}\right)$ (3.51). Solución de MN.

relaciona con la escala dinámica generada de la teoría de gauge dual.

En las figuras 3.7 y 3.8 se grafican las funciones $\bar{L}\left(r_{0}\right)$ y $\bar{E}\left(r_{0}\right)$ provenientes de las ecuaciones $(3.50)$ y (3.51). La divergencia $L \rightarrow \infty$ para $r_{0} \rightarrow 0$ en la figura 3.7 se debe a que $\left.\frac{d}{d r}\left(e^{2 \phi(r)}\right)\right|_{r=0}=0$ (ver [122] para una discusión sobre este echo).

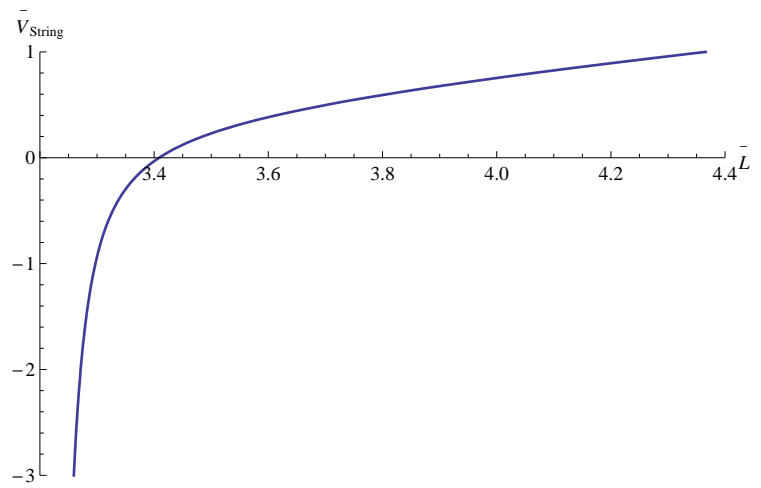

Figura 3.9: Energía potencial $V_{\text {string }}(L)$ para el par $q \bar{q}$ obtenida a partir de la solución de MaldacenaNuñez eliminando numéricamente $r_{0}$ de (3.50)-(3.51). Nótese el cambio en el comportamiento de la función pasando de ser tipo Coulomb a lineal.

El resultado para el potencial $V_{\text {string }}(L)$ en la figura 3.9 es gratificante, pero el comportamiento lineal ocurre para configuraciones que tienen energías por encima de cero. Una pregunta que surge es si debemos confiarle a la solución cuando $V_{\text {string }}>0$ (ver el último párrafo de la sección 3.2.2). La sustracción en (3.51) corresponde a un par de cuerdas rectas expandidas a lo largo de la dirección radial con las demás coordenadas espaciales fijas. Al ser regular el fondo gravitatorio, las cuerdas no pueden terminar en ningún punto en el interior y la única posibilidad para obtener una solución de referencia suave es ubicar los extremos de la cuerda antipodalmente en la coordenada $\varphi$ y teniendo 
ambos la misma coordenada $x_{1}$ (ver la linea azul horizontal en la fig.3.10 y la discusión relacionada en la sección 3.2.1). Se concluye que aunque el confinamiento lineal ocurre para configuraciones con $E_{q \bar{q}}>0$, la solución es estable y no puede decaer al estado de referencia $E_{q \bar{q}}=0$ ya que las dos configuraciones a comparar en (3.51) satisfacen diferentes condiciones de borde. En la figura 3.10 se dibujan las hojas de mundo relevantes para el calculo del valor de expectación del lazo de Wilson rectangular en fondos de gravedad suaves.

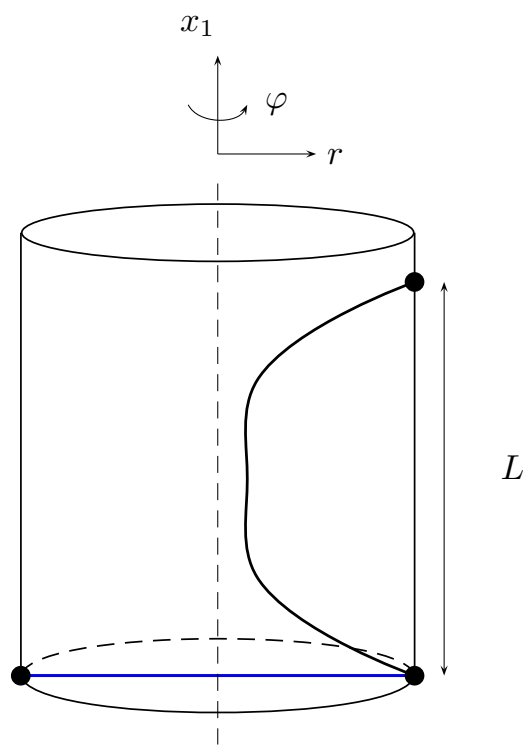

Figura 3.10: Geodésicas empleadas para el cálculo de lazos de Wilson rectangulares en fondos gravitatorios suaves. La curva negra vertical muestra la solución en forma de U, la linea azul horizontal es el estado de referencia con respecto al cual comparar la energía de la linea negra. Las configuraciones negra y azul satisfacen diferentes condiciones de borde.

\subsubsection{Klebanov-Strassler}

Este fondo gravitatorio describe una (quiver) teoría de gauge $\mathcal{N}=1$ con campos de materia en la representación fundamental del grupo de gauge $S U(N+M) \times S U(N)$. La configuración de branas de prueba que conducen a esta geometría es: $N D 3$ branas en el vértice del conifold singular más $M D 5$-branas enrolladas en la $S^{2}$ del conifold y compartiendo las restantes 3 dimensiones con la D3. La solución esta soportada por un dilatón constante $\phi=\phi_{0}$, el cual puede ser fijado para tener $g_{s} \ll 1$ en todo el espacio (al contrario que en la solución de $\mathrm{MN}$ ). El flujo $\mathcal{N}=1$ $S U(N+M) \times S U(N) \rightarrow S U(M)$ que se realiza a través de una cascada de dualidades de Seiberg en la teoría de gauge (ver [123]) se manifiesta en la geometría por medio de la variación del flujo de la 5-forma. 


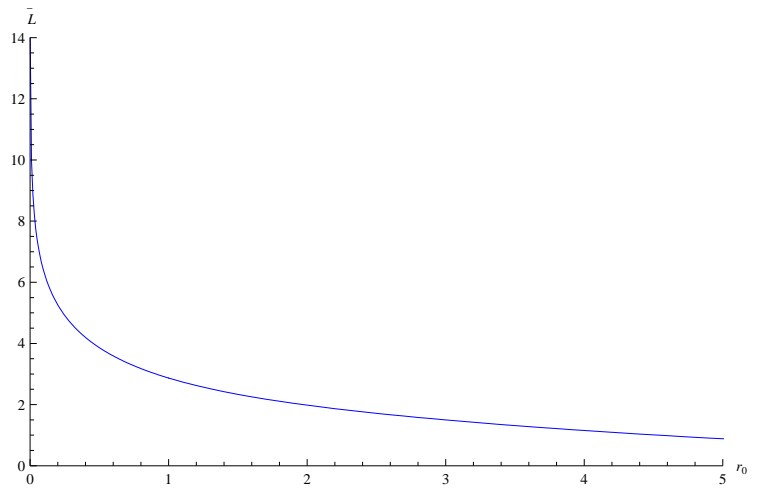

Figura 3.11: $\bar{L}\left(r_{0}\right)$ (3.60). Solución de KS.

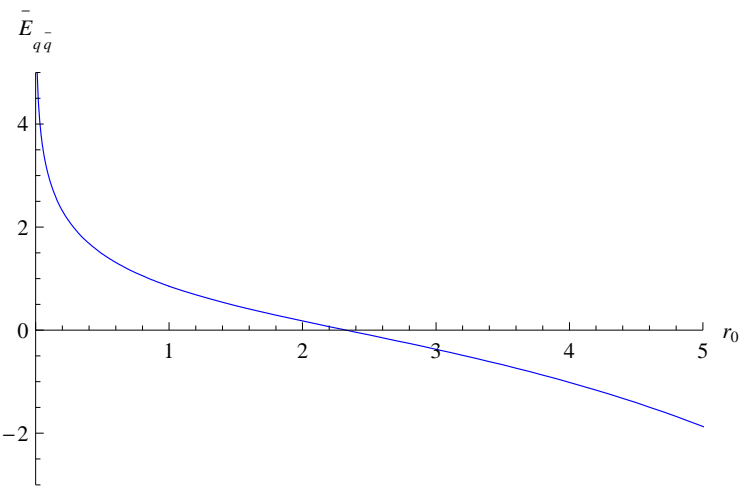

Figura 3.12: $\bar{E}_{q \bar{q}}\left(r_{0}\right)(3.61)$. Solución de KS.

La métrica es [7]

$$
d s^{2}=g_{s} \alpha^{\prime} M\left[h^{-\frac{1}{2}}(r)\left(-d t^{2}+d x_{i} d x_{i}\right)+h^{\frac{1}{2}}(r) d s_{6}^{2}\right]
$$

La geometría del conifold deformado $d s_{6}$ se puede escribir como

$$
d s_{6}^{2}=\frac{1}{2} K(r)\left[\frac{\left(d r^{2}+\left(g^{5}\right)^{2}\right)}{3 K^{3}(r)}+\cosh ^{2} \frac{r}{2}\left(\left(g^{3}\right)^{2}+\left(g^{4}\right)^{2}\right)+\sinh ^{2} \frac{r}{2}\left(\left(g^{1}\right)^{2}+\left(g^{2}\right)^{2}\right)\right],
$$

en donde

$$
K(r)=\frac{[\sinh (2 r)-2 r]^{\frac{1}{3}}}{2^{\frac{1}{3}} \sinh r}
$$

y las $g_{i}$ están definidas por

$$
g^{1}=\frac{e^{1}-e^{3}}{\sqrt{2}}, \quad g^{2}=\frac{e^{2}-e^{4}}{\sqrt{2}}, \quad g^{3}=\frac{e^{1}+e^{3}}{\sqrt{2}}, \quad g^{4}=\frac{e^{2}+e^{4}}{\sqrt{2}}, \quad g^{5}=e^{5},
$$

con

$$
\begin{gathered}
e^{1}=-\sin \theta_{1} d \phi_{1}, \quad e^{2}=d \theta_{1}, \quad e^{3}=-\sin \psi d \theta_{2}+\cos \psi \sin \theta_{2} d \phi_{2}, \\
e^{4}=\cos \psi d \theta_{2}+\sin \psi \sin \theta_{2} d \phi_{2}, \quad e^{5}=d \psi+\cos \theta_{1} d \phi_{1}+\cos \theta_{2} d \phi_{2} .
\end{gathered}
$$

Las coordenadas en (3.54) son adimensionales, las coordenadas de la teoría de gauge $t, x_{i}$ escalean como $\frac{g_{s} M \alpha^{\prime}}{\ell_{c f}}$, y la escala de la coordenada holográfica $r$ esta fijada por $\ell_{c f}$. El factor $h(r)$ toma la forma

$$
h(r)=2^{\frac{2}{3}} \int_{r}^{\infty} d x \frac{x \operatorname{coth} x-1}{\sinh ^{2} x}(\sinh 2 x-2 x)^{\frac{1}{3}} .
$$

El fondo gravitatorio (3.54) esta soportado en parte por un $B_{\mu \nu}$ no trivial pero la forma de embeber la cuerda que consideramos hace que este no contribuya en los cálculos [113]. Las funciones en (3.4) están dadas por $f^{2}(r)=\frac{1}{h(r)}, g^{2}(r)=\frac{1}{6 K^{2}(r)}$. Las expresiones adimensionales para la longitud (3.9) y la energía (3.15) son

$$
\bar{L}\left(r_{0}\right)=2 \int_{r_{0}}^{\infty} \frac{d r}{\sqrt{6} K(r)} \frac{h(r)}{\sqrt{h\left(r_{0}\right)-h(r)}} .
$$




$$
\bar{E}_{q \bar{q}}\left(r_{0}\right)=\frac{g_{s} M}{\pi}\left[\int_{r_{0}}^{\infty} \frac{d r}{\sqrt{6} K(r)} \frac{\sqrt{h\left(r_{0}\right)}}{\sqrt{h\left(r_{0}\right)-h(r)}}-\int_{0}^{r_{0}} \frac{d r}{\sqrt{6} K(r)}\right] .
$$

En las figuras 3.11 y 3.12 se graficaron estas dos últimas expresiones. Al igual que en el caso de MN, se espera encontrar una divergencia en $r_{0}=0$ debido a que $\left.\frac{d h(r)}{d r}\right|_{r=0}=0$. Eliminando numéricamente $r_{0}$ de las ecuaciones (3.60)-(3.61) se grafica en la figura 3.13 la función $V_{\text {string }}(L)$. Como se puede observar fácilmente, existe una relación lineal para el potencial de interacción para valores de $L \gg 1$. Procediendo como en (3.52) encontramos que la tensión de la cuerda confinante es

$$
T_{\text {string }}=\frac{1}{2 \pi \alpha^{\prime}} \frac{\ell_{c f}^{2}}{g_{s} \alpha^{\prime} M \sqrt{h_{0}}},
$$

en donde $h_{0}=h(0) \simeq 1,1398$. Como en el caso de MN, la contribución dominante al area mínima (3.1), en el límite de $L$ grande, proviene de la región $r \approx 0$. De nuevo, hay un problema relacionado a cuándo se le debe creer a configuraciones con $E_{q \bar{q}}>0$, como se discutió al final de la sección anterior y se dibujó en la fig. 3.10 las soluciones con $E_{q \bar{q}}>0$ son clásicamente estables.

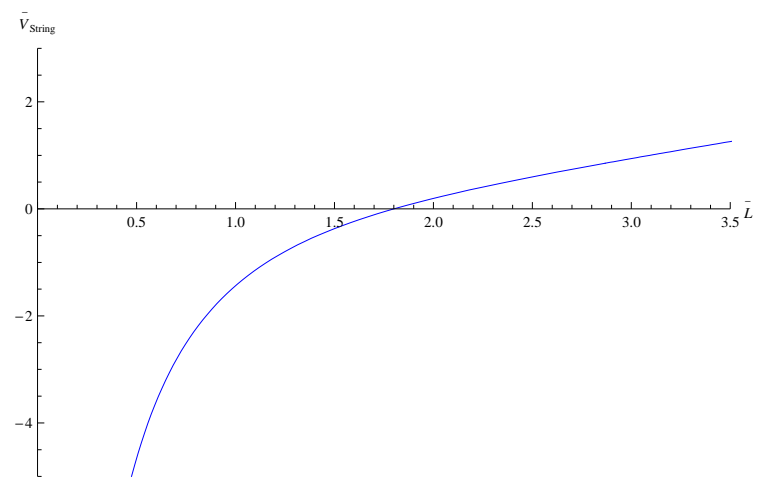

Figura 3.13: $V_{\text {string }}(L)$ para un lazo de Wilson rectangular sobre el fondo de gravedad de KS.

\subsubsection{Maldacena-Núñez generalizado}

Este tipo de fondos gravitatorios fueron obtenidos en [114] generalizando la solución descripta en la sección 3.2.3. Estas soluciones fueron ampliamente discutidas en [116] y se interpretan como duales a teorías de gauge mínimamente supersimétricas que contienen operadores irrelevantes de dimensión 6. El operador cambia drásticamente el desarrollo ultravioleta (UV) de las teorías tomando la solución " afuera "del límite de horizonte cercano de las D5-branas que generan la geometría. El análisis en [116] muestra que asintóticamente, $r$ grande, la solución es el producto de un espacio de Minkowski en 4 dimensiones con el conifold deformado. 


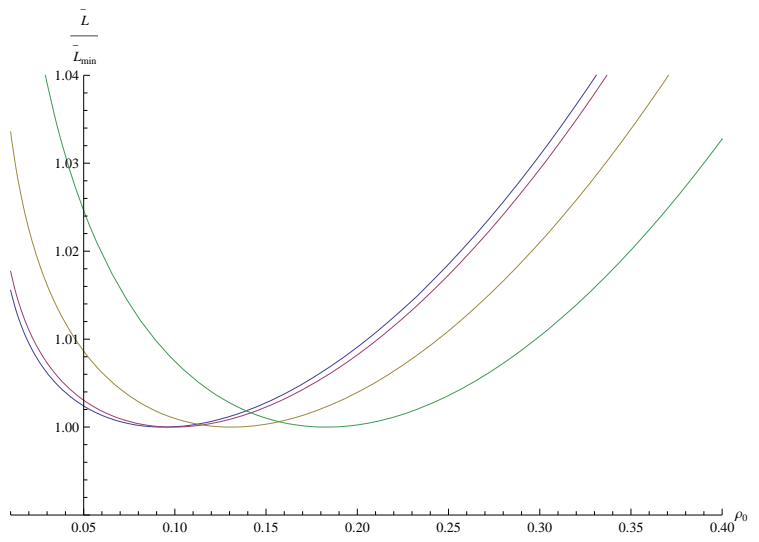

Figura 3.14: $L\left(\rho_{0}\right)(3.68)$. Las curvas azul, violeta, amarilla y verde se corresponden con los valores $\mu=-1,8,-1,5,-1,-, 8\left(\rho_{\infty}=7\right)$. Se observa una distancia de separación mínima para los quarks.

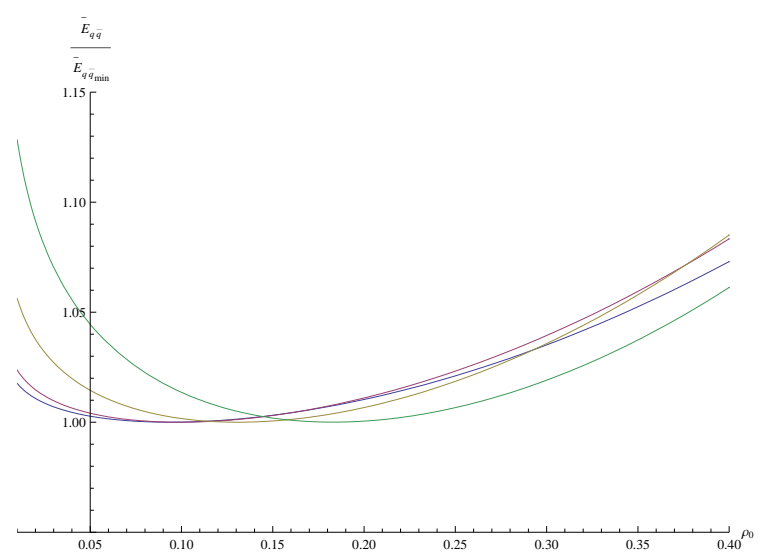

Figura 3.15: $E\left(r_{0}\right)$ (3.69). Los colores se corresponden con los valores de $\mu$ de la fig. 3.14 .

La métrica es [114]

$$
\begin{aligned}
d s^{2}= & g_{s} \alpha^{\prime} N e^{4 f(r)}\left[-d t^{2}+d x_{i} d x_{i}+d r^{2}+e^{2 h(r)}\left(d \theta^{2}+\sin ^{2} \theta d \varphi^{2}\right)\right. \\
& \left.+\frac{e^{2 g(r)}}{4}\left(\left(w_{1}+a(r) d \theta\right)^{2}+\left(w_{2}-a(r) \sin \theta d \varphi\right)^{2}\right)+\frac{e^{2 k(r)}}{4}\left(w_{3}+\cos \theta d \varphi\right)^{2}\right] .
\end{aligned}
$$

Realizando el cambio de coordenadas $d \rho \equiv e^{-k(r)} d r$, las funciones $a, k$ y $f$ satisfacen

$$
\begin{aligned}
\partial_{\rho} a & =\frac{-2}{-1+2 \rho \operatorname{coth} 2 \rho}\left[e^{2 k} \frac{(a \cosh 2 \rho-1)^{2}}{\sinh 2 \rho}+a(2 \rho-a \sinh 2 \rho)\right] \\
\partial_{\rho} k & =\frac{2\left(1+a^{2}-2 a \cosh 2 \rho\right)^{-1}}{-1+2 \rho \operatorname{coth} 2 \rho}\left[e^{2 k} a \sinh 2 \rho(a \cosh 2 \rho-1)+\left(2 \rho-4 a \rho \cosh 2 \rho+\frac{a^{2}}{2} \sinh 4 \rho\right)\right] \\
\partial_{\rho} f & =-\frac{1}{4 \sinh ^{2} 2 \rho}\left[\frac{(1-a \cosh 2 \rho)^{2}(-4 \rho+\sinh 4 \rho)}{\left(1+a^{2}-2 a \cosh 2 \rho\right)(-1+2 \rho \operatorname{coth} 2 \rho)}\right],
\end{aligned}
$$

y las funciones $g(\rho), h(\rho)$ en (3.63) están dadas por

$$
e^{2 g}=\frac{b \cosh 2 \rho-1}{a \cosh 2 \rho-1}, \quad e^{2 h}=\frac{e^{2 g}}{4}\left(2 a \cosh 2 \rho-1-a^{2}\right), \quad \text { con } b(\rho)=\frac{2 \rho}{\sinh 2 \rho} .
$$

Las primeras dos ecuaciones diferenciales en (3.64) tienen una familia de soluciones regulares dependientes de un solo parámetro. Para pequeños valores de $r$ se encuentra [114]

$$
a(\rho)=1+\mu \rho^{2}+\ldots, \quad e^{2 k(\rho)}=\frac{4}{6+3 \mu}-\frac{20+36 \mu+9 \mu^{2}}{15(2+\mu)} \rho^{2}+\ldots
$$

con $\mu$ tomando valores en el intervalo $\left(-2,-\frac{2}{3}\right)$. Insertando (3.66) en la tercera ecuación de (3.64) y en (3.65) se obtiene

$$
e^{2 g(\rho)}=\frac{4}{6+3 \mu}+\ldots, \quad e^{2 h(\rho)}=\frac{4 \rho^{2}}{6+3 \mu}+\ldots, \quad e^{2 f(\rho)}=1+\frac{(2+\mu)^{2}}{8} \rho^{2}+\ldots
$$




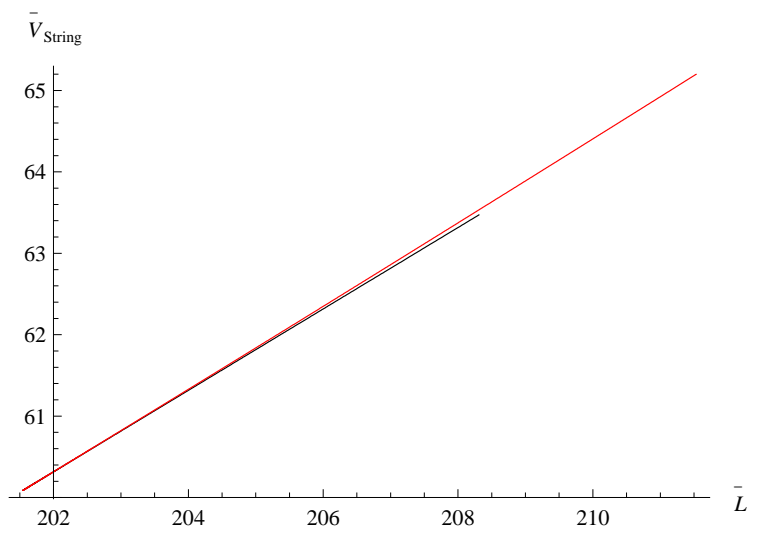

Figura 3.16: $V_{\text {string }}(L)$ bivaluada para el caso de $\operatorname{gMN}$ con $\mu=-1$. La curva superior es la rama no física correspondiente a soluciones de cuerda a la derecha del mínimo en la figura 3.14. La curva no alcanza el origen, manifestando así la existencia de una longitud separación mínima entre quarks.

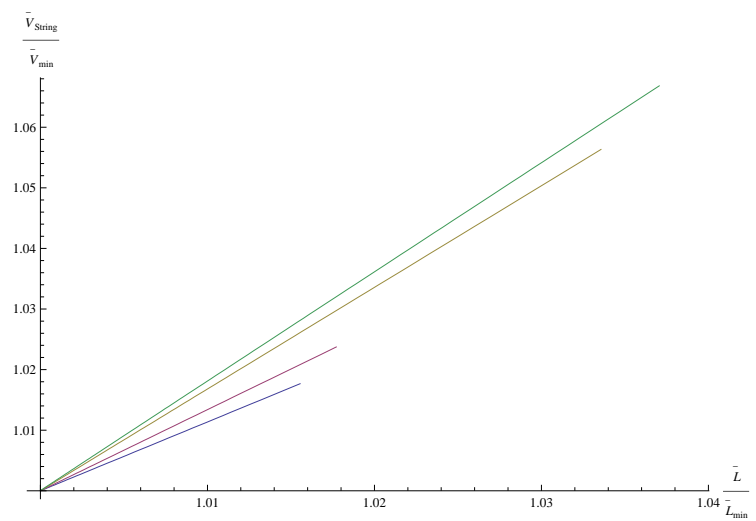

Figura 3.17: $\bar{V}_{\text {string }}(L)$ normalizado y lineal para la rama física (izquierda) de la figura 3.14. Los colores se corresponden con los de la fig. 3.14.

La constante arbitraria para $f$ proveniente de (3.64) fue factorizada como $g_{s}$ in (3.63). Los valores límites para $\mu$ dan resultados conocidos: el caso con $\mu=-\frac{2}{3}$ reproduce la solución de MN estudiada en la sección 3.2 .3 con $\phi=4 f$ ( $k=$ const.), y el caso con $\mu=-2$ nos conduce al producto del espacio de Minkowski en 4 dimensiones con el conifold deformado ( $\phi$ en este caso es constante). Finalmente, el límite $\rho \rightarrow \infty$ de todas las soluciones (excepto $\mu=-\frac{2}{3}$ ) tiene al conifold deformado como límite asintótico (ver [114] para más detalles).

La expresiones para la longitud (3.9) y la energía (3.15) quedan

$$
\begin{gathered}
\bar{L}\left(\rho_{0}\right)=2 \int_{\rho_{0}}^{\rho_{\infty}} \frac{e^{4 f\left(\rho_{0}\right)}}{\sqrt{e^{8 f(\rho)}-e^{8 f\left(\rho_{0}\right)}}} e^{k(\rho)} d \rho \\
\bar{E}_{q \bar{q}}\left(\rho_{0}\right)=\frac{N g_{s}}{\pi}\left[\int_{\rho_{0}}^{\rho_{\infty}} \frac{e^{8 f(\rho)}}{\sqrt{e^{8 f(\rho)}-e^{8 f\left(\rho_{0}\right)}}} e^{k(\rho)} d \rho-\int_{0}^{\rho_{\infty}} e^{4 f(\rho)} e^{k(\rho)} d \rho\right] .
\end{gathered}
$$

Nótese que la integración a lo largo de la coordenada radial en ambas expresiones se extiende hasta una distancia finita $\rho_{\infty}$. La razón, estudiada en [114], proviene de notar que (3.68) es divergente 8 . Se debe tener en cuenta que el cálculo del lado de cuerdas se corresponde con estudiar la teoría de gauge dual con quarks muy masivos para $\rho_{\infty} \gg 1$ (pero no infinitamente masivos). Además, como los extremos de la cuerda están fijos a una distancia radial finita, se puede chequear que la cuerda de prueba no alcanza a lo largo de la dirección normal a la teoría de gauge definida sobre la brana.

En las figuras 3.14 y 3.15 se grafican las soluciones numéricas de (3.68) y (3.69) para varios valores de $\mu$. La figura (3.14) muestra que $L\left(\rho_{0}\right)$ presenta un mínimo global para todos los valores

\footnotetext{
${ }^{8}$ Ver [122] para encontrar una discusión sobre las divergencias cuando se calcula la función longitud $L\left(r_{0}\right)$.
} 
de $\mu$ (excepto para $\mu \neq-\frac{2}{3}$ ). En otras palabras, no existen soluciones para distancias de separación $L<L_{\text {min }}$. Es interesante notar que el valor mínimo de $L$ se encuentra para todos los valores de $\mu$ en una región pequeña en la coordenada $\rho$ cerca del origen. Basándonos en las consideraciones de concavidad discutidas al final de la sección 3.1 se espera que la hoja de mundo de la cuerda a la izquierda del mínimo $\rho_{c}$ tenga un sentido físico bien definido (que sea estable) y que la rama de la derecha de $\rho_{c}$ sea no física (inestable). En la sección siguiente voy a mostrar que esto es lo que sucede cuando se analizan fluctuaciones cuadráticas alrededor de las soluciones. Se encuentran autovalores negativos para la rama de soluciones con $L^{\prime}\left(\rho_{0}\right)>0$.

En las figuras (3.16) y (3.17) se grafica la relación $V_{\text {string }}(L)$ para este fondo gravitatorio en donde se puede observar un confinamiento lineal. La figura (3.16) muestra la función bivaluada $V_{\text {string }}(L)$ para $\mu=-1$, la rama superior (no física, en rojo) corresponde a configuraciones de cuerda a la derecha del mínimo de la fig. (3.14). En la figura 3.17 se muestra la función $V_{\text {string }}(L)$ para las ramas físicas de la fig.3.14 y para diferentes valores de $\mu$. Procediendo como en (3.52) se encuentra que todas las soluciones conducen a una tensión de la cuerda independiente de $\mu$

$$
T_{\text {string }}=\frac{g_{s}}{2 \pi \alpha^{\prime}}
$$

\subsection{Análisis de Estabilidad}

En esta sección se va a estudiar, para los fondos gravitatorios presentados en la sección previa, el problema de autovalores definido por la ecuación de movimiento (3.22) para fluctuaciones en el plano en el gauge $r$. Estoy interesado en encontrar los modos inestables. Por las razones discutidas en el final de la sección 3.1, el objetivo es mostrar que dichos autovalores negativos $\left(\omega^{2}<0\right)$ se producen para soluciones de cuerda en regiones en donde $L^{\prime}\left(r_{0}\right)>0$. Se estudiarán soluciones pares, esto significa que en (3.25) las condiciones iniciales en el tip serán $C_{1}^{\prime}=1$ y $C_{0}^{\prime}=0{ }^{9}$. Estas condiciones van a ser implementadas numéricamente como (ver (3.31))

$$
\begin{array}{rlrl}
\delta x_{1}(r)+2\left(r-r_{0}\right) \frac{d \delta x_{1}(r)}{d r} & =0, & & r \rightarrow r_{0} \\
\sqrt{r-r_{0}} \delta x_{1}(r)=1, & r \rightarrow r_{0} .
\end{array}
$$

Resolviendo numéricamente, los autovalores $\omega^{2}$ permitidos para (3.22) se obtienen pidiendo que $\delta x_{1}(r)$ sea una solución normalizable

$$
\delta x_{1}(r)=0, \quad r \rightarrow \infty .
$$

\footnotetext{
${ }^{9}$ La soluciones pares corresponden a elegir arbitrariamente $C_{1}^{\prime}$ en el tip, su valor fija la normalización de la solución.
} 


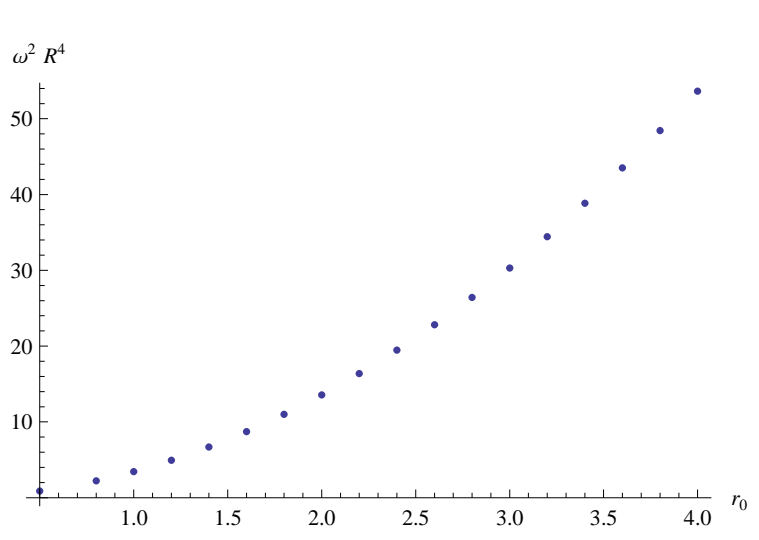

Figura 3.18: Autovalor mínimo $\omega^{2}$ de (3.76) que conduce a una solución normalizable como función de $r_{0}$.

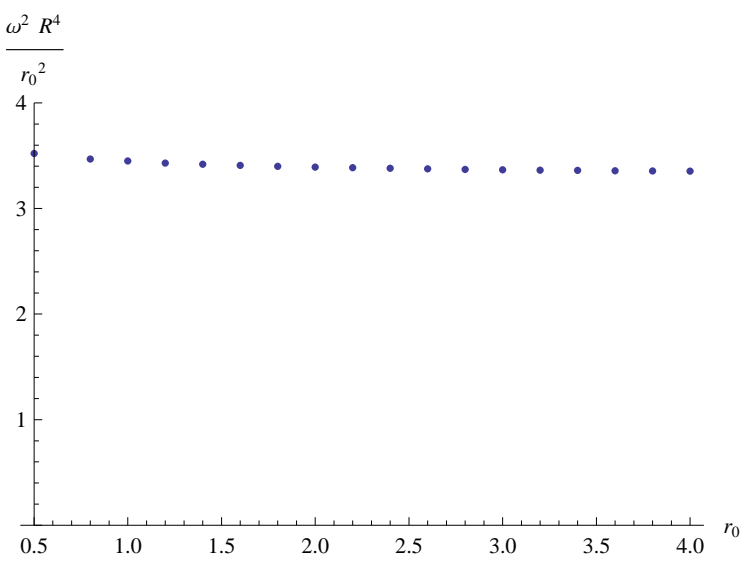

Figura 3.19: Autovalor mínimo propiamente normalizado de la fig. 3.18 como función de $r_{0}$ $(\operatorname{ver}(3.77))$.

Por completitud se van a escribir las relaciones entre los modos cero y los puntos críticos de la función $L\left(r_{0}\right)$ [99]. La solución de modo cero de (3.22) puede inmediatamente escribirse como

$$
\delta x_{1}^{(0)}(r)=C \int_{r}^{\infty} d \bar{r} \frac{g(\bar{r}) f(\bar{r})}{\left(f^{2}(\bar{r})-f^{2}\left(r_{0}\right)\right)^{\frac{3}{2}}}+C^{\prime}
$$

en donde $C^{\prime}$ y $C$ son las constantes de integración, se fija $C^{\prime}=0$ para obtener una solución normalizable y se escoge $C^{\prime}=1$. Integrando por partes en (3.73) y usando (3.10) se obtiene

$$
\begin{aligned}
\delta x_{1}^{(0)}(r) & =-\int_{r}^{\infty} d \bar{r} \frac{g(\bar{r})}{f^{\prime}(\bar{r})} \frac{d}{d \bar{r}}\left(\frac{1}{\sqrt{f^{2}(\bar{r})-f^{2}\left(r_{0}\right)}}\right) \\
& =\frac{g(r)}{f^{\prime}(r) \sqrt{f^{2}(r)-f^{2}\left(r_{0}\right)}}+\frac{L^{\prime}(r)}{2 f^{\prime}(r)} .
\end{aligned}
$$

Expandiendo esta última expresión alrededor del tip $r=r_{0}$ se tiene

$$
\delta x_{1}^{(0)}(r)=\frac{g\left(r_{0}\right)}{\sqrt{2}\left(f^{\prime}\left(r_{0}\right)\right)^{\frac{3}{2}}} \frac{1}{\sqrt{r-r_{0}}}+\frac{L^{\prime}\left(r_{0}\right)}{2 f^{\prime}\left(r_{0}\right)}+\mathcal{O}\left(\sqrt{r-r_{0}}\right) .
$$

Por lo general, el primer factor en el lado derecho de (3.75) es no nulo, luego, una condición necesaria y suficiente para obtener una solución par con modo cero (ver (3.71)) requiere que el segundo termino en (3.75) se anule, ó, equivalentemente, $r_{0}$ debe ser un punto crítico de la función longitud $L\left(r_{0}\right)[99]$.

\subsection{1. $A d S_{5}$}

Las ecuaciones de movimiento (3.22) para la fluctuaciones en el caso de AdS en coordenadas de Poincaré (3.33) toma la forma

$$
\left[\frac{d}{d r}\left(\frac{\left(r^{4}-r_{0}^{4}\right)^{\frac{3}{2}}}{r^{2}} \frac{d}{d r}\right)+\omega^{2} R^{4} \frac{\sqrt{r^{4}-r_{0}^{4}}}{r^{2}}\right] \delta x_{1}(r)=0 \quad 0<r_{0} \leq r<\infty .
$$


La invarianza ante dilataciones nos permite factorizar la dependencia en $r_{0}$. Haciendo $r=r_{0} \rho$ se obtiene

$$
\left[\frac{d}{d \rho}\left(\frac{\left(\rho^{4}-1\right)^{\frac{3}{2}}}{\rho^{2}} \frac{d}{d \rho}\right)+\frac{\omega^{2} R^{4}}{r_{0}^{2}} \frac{\sqrt{\rho^{4}-1}}{\rho^{2}}\right] \delta x_{1}(\rho)=0 .
$$

El desarrollo asintótico $(\rho \rightarrow \infty)$ de $(3.77)$ es

$$
\left[\frac{d}{d \rho}\left(\rho^{4} \frac{d}{d \rho}\right)+\frac{\omega^{2} R^{4}}{r_{0}^{2}}\right] \delta x_{1}(\rho) \approx 0, \quad \rho \gg 1
$$

cuya solución se escribe

$$
\delta x_{1}(\rho) \approx \alpha_{0}+\frac{\alpha_{1}}{\rho^{3}}, \quad \rho \gg 1,
$$

en donde $\alpha_{0}, \alpha_{1}$ son constantes de integración. El comportamiento (3.79) implica que existirán soluciones normalizables $\left(\alpha_{0}=0\right)$ para valores particulares (discretos) de $\omega_{n}^{2}$.

Con el objeto de testear el método de shooting empleado, se integrará numéricamente (3.76) para diferentes valores de $r_{0}$ y se determinará el autovalor mínimo $\omega^{2}$ compatible con un solución normalizable. En la figura 3.18 se grafican estos $\omega^{2}$ como función de $r_{0}$. Los autovalores son positivos para todo valor de $r_{0}$, lo cual señala la estabilidad de la configuración de cuerda. En la figura 3.19 se muestra la esperada independencia en $r_{0}$ de los modos apropiadamente normalizados (ver (3.77)). En la siguiente tabla se muestran los primeros autovalores correspondientes a condiciones de borde pares en el tip.

\begin{tabular}{|c||c|}
\hline & $\omega_{n}^{2} R^{4} / r_{0}^{2}$ \\
\hline$n=1$ & 3.450 \\
$n=3$ & 22.113 \\
$n=5$ & 52.325 \\
$n=7$ & 94.558 \\
$n=9$ & 148.845 \\
\hline
\end{tabular}

En la sección 3.4 se probará la estabilidad de la configuración, transformando la ecuación diferencial (3.76) en una ecuación tipo Schrodinger (ver apéndice A.1).

\subsubsection{D3-branas no extremales}

La ecuación de movimiento para la fluctuación (3.22) en el fondo gravitatorio (3.43) toma la forma

$$
\left[\frac{d}{d \rho}\left(\frac{\left(\rho^{4}-\rho_{0}^{4}\right)^{\frac{3}{2}}}{\sqrt{\rho^{4}-1}} \frac{d}{d \rho}\right)+\frac{\omega^{2} R^{4}}{\mu^{2}} \frac{\rho^{4} \sqrt{\rho^{4}-\rho_{0}^{4}}}{\left(\rho^{4}-1\right)^{\frac{3}{2}}}\right] \delta x_{1}(\rho)=0, \quad 1<\rho_{0} \leq \rho<\infty .
$$

El fondo de gravedad (3.43) tiende asintóticamente a AdS y por lo tanto el comportamiento asintótico $(\rho \rightarrow \infty)$ de la solución de (3.80) esta dado por (3.79). Como en el caso anterior, se espera encontrar un conjunto de autovalores que conducen a soluciones normalizables. 


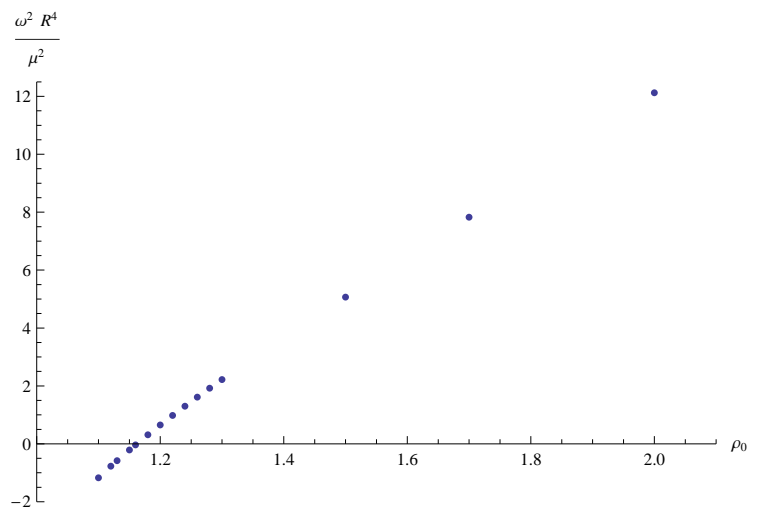

Figura 3.20: Minimo autovalor $\omega^{2}$ de (3.80) que conduce a una solución normalizable como función de $\rho_{0}$. Existe un modo cero para $\rho_{0} \simeq 1,177$. La solución clásica con $\rho_{0}<1,177$ es inestable ante perturbaciones lineales.

Se grafica en la figura 3.20 el mínimo autovalor encontrado numéricamente resolviendo (3.80) y que permite soluciones normalizables. Aparece un modo cero precisamente en el punto crítico de la función longitud $L\left(\rho_{0}\right)$, esto es para $\rho_{0} \simeq 1,177$ (ver fig.3.4), en acuerdo con (3.75).

Se concluye que la rama (izquierda) en la figura 3.4 que posee $L^{\prime}\left(\rho_{0}\right)>0$ es inestable ante perturbaciones lineales. Finalmente, nótese que el análisis numérico indica que las soluciones de la rama derecha de la figura 3.4 , para las cuales $L^{\prime}\left(\rho_{0}\right)<0$, son estables ante perturbaciones lineales. Sin embargo, como se discutió al final de la sección 3.2.2 se espera que la solución con $1,177<\rho_{0}<1,524$ sea metaestable, decayendo a un par de quarks libres.

\subsubsection{Maldacena-Núñez}

La ecuación de movimiento para la fluctuación en el plano en la geometría de Maldacena-Nuñez (3.47) toma la forma (3.22)

$$
\left[\frac{d}{d r}\left(\frac{\left(e^{2 \phi(r)}-e^{2 \phi\left(r_{0}\right)}\right)^{\frac{3}{2}}}{e^{2 \phi(r)}} \frac{d}{d r}\right)+\bar{\omega}^{2} \sqrt{e^{2 \phi(r)}-e^{2 \phi\left(r_{0}\right)}}\right] \delta x_{1}(r)=0, \quad 0<r_{0} \leq r<\infty .
$$

en donde $\bar{\omega}^{2}=\omega^{2} \alpha^{\prime} N$. Se analizará ahora el desarrollo asintótico de (3.81) para ver cuando debiese uno esperar un espectro discreto o no. En el límite $r \rightarrow \infty$ la ecuación de movimiento para $\delta x_{1}(r)$ queda

$$
\left[\frac{d}{d r}\left(e^{r} r^{-\frac{1}{4}} \frac{d}{d r}\right)+\bar{\omega}^{2} e^{r} r^{-\frac{1}{4}}\right] \delta x_{1}(r)=0, \quad r \gg 1,
$$

en donde se usó que $e^{2 \phi(r)} \rightarrow e^{2 r} r^{-\frac{1}{2}}$ para $r \gg 1$. Esta última ecuación puede escribirse como

$$
\left[\frac{d^{2}}{d r^{2}}+\left(1-\frac{1}{4 r}\right) \frac{d}{d r}+\bar{\omega}^{2}\right] \delta x_{1}(r)=0, \quad r \gg 1
$$


El término $r^{-1}$ puede omitirse en el límite de $r$ grande y la solución asintótica para (3.81) es

$$
\delta x_{1}(r) \simeq e^{-\frac{1}{2} r}\left(\beta_{0} e^{r \alpha}+\beta_{1} e^{-r \alpha}\right), \quad r \gg 1,
$$

en donde $\alpha=\frac{\sqrt{1-4 \bar{\omega}^{2}}}{2}$. A partir de (3.83) se sigue que cualquier $\bar{\omega}^{2}>0$ conduce a soluciones normalizables, el espectro de fluctuaciones es por lo tanto continuo. En el caso $\bar{\omega}^{2} \leq 0\left(\alpha \geq \frac{1}{2}\right)$, $\beta_{0}$ debe ser fijada a cero y se tiene la posibilidad encontrar un espectro discreto de autovalores negativos. El análisis numérico no encontró ningún autovalor normalizable negativo, sugiriendo la estabilidad de la configuración clásica y en acuerdo con la condición de concavidad (3.17).

En la sección 3.4 se mostrará que no existen autovalores negativos a partir del estudio de la ecuación de Schrödinger de (3.81) (ver apéndice A.1).

\subsubsection{Klebanov-Strassler}

En este caso, la ecuación de movimiento para la fluctuación toma la forma

$$
\left[\frac{d}{d r}\left(\frac{K(r)}{h(r)}\left(1-\frac{h(r)}{h\left(r_{0}\right)}\right)^{\frac{3}{2}} \frac{d}{d r}\right)+\bar{\omega}^{2} \frac{1}{6 K(r)} \sqrt{1-\frac{h(r)}{h\left(r_{0}\right)}}\right] \delta x_{1}(r)=0, \quad 0<r_{0} \leq r<\infty,
$$

con $\bar{\omega}^{2}$ adimensional y $K(r)$ y $h(r)$ dadas por (3.56) y (3.59) respectivamente. Del límite $r \rightarrow \infty$ de $K(r)$ y $h(r)$ se obtiene

$$
\left[\frac{d}{d r}\left(\frac{e^{r}}{r} \frac{d}{d r}\right)+\bar{\omega}^{2} \frac{e^{\frac{r}{3}}}{2^{\frac{4}{3}}}\right] \delta x_{1}(r)=0, \quad r \gg 1
$$

con lo cual da

$$
\left[\frac{d^{2}}{d r^{2}}+\left(1-\frac{1}{r}\right) \frac{d}{d r}+\bar{\omega}^{2} \frac{r e^{-\frac{2}{3} r}}{2^{\frac{4}{3}}}\right] \delta x_{1}(r)=0, \quad r \gg 1 .
$$

En el límite de valores grandes para $r$ el término con $r^{-1}$ y el último término en (3.86) pueden ser omitidos y la fluctuación asintóticamente tiende a

$$
\delta x_{1}(r) \simeq \alpha_{0}+\alpha_{1} e^{-r}, \quad r \gg 1 .
$$

La constante de integración $\alpha_{0}$ debe ser fijada a cero para obtener soluciones normalizables y por lo tanto se espera obtener un conjunto discreto de autovalores. En la figura (3.21) se grafican (como función de $r_{0}$ ) los autovalores más bajos de (3.84) tales que que conducen a soluciones normalizables. No se han encontrado numéricamente autovalores negativos. En la sección 3.4 se probará la estabilidad de la solución clásica transformando (3.84) en una ecuación del tipo Schrödinger mostrando que no pueden existir modos negativos (ver apéndice A.1).

\subsubsection{Maldacena-Núñez generalizada}

La ecuación de movimiento para la fluctuación $\delta x_{1}(\rho)$ en la geometría (3.63) es

$$
\left[\frac{d}{d \rho}\left(\frac{\left(e^{8 f(\rho)}-e^{8 f\left(\rho_{0}\right)}\right)^{\frac{3}{2}}}{e^{8 f(\rho)+k(\rho)}} \frac{d}{d \rho}\right)+\bar{\omega}^{2} e^{k(\rho)} \sqrt{e^{8 f(\rho)}-e^{8 f\left(\rho_{0}\right)}}\right] \delta x_{1}(\rho)=0, \quad 0<\rho_{0} \leq \rho<\infty .
$$




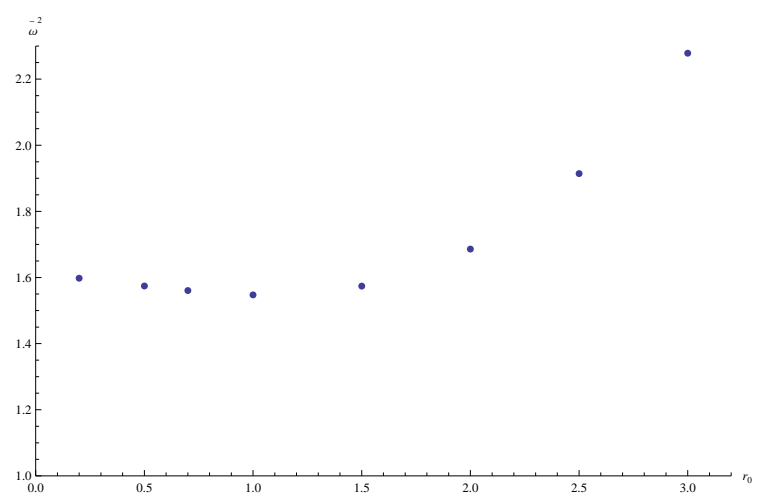

Figura 3.21: Soluciones numéricas para los menores $\omega^{2}$ que permiten encontrar soluciones normalizables como función de $r_{0}$ para el fondo gravitatorio de Klebanov-Strassler. No se han encontrado modos negativos.

En el caso $\mu=-\frac{2}{3}(k(\rho)=$ const.) se recupera la ecuación (3.81) para el fondo de MaldacenaNuñez (a partir de ahora se considerará $\mu \neq-\frac{2}{3}$ ). En el límite de $\rho$ grande, la solución de gMN tiende al conifold deformado y la función $f$ toma un valor constante $f_{\infty}$, el desarrollo asintótico queda dado por

$$
\left[e^{-k(\rho)} \frac{d}{d \rho}\left(e^{-k(\rho)} \frac{d}{d \rho}\right)+\bar{\omega}^{2} \frac{e^{8 f_{\infty}}}{e^{8 f_{\infty}}-e^{8 f\left(\rho_{0}\right)}}\right] \delta x_{1}(\rho)=0, \quad \rho \gg 1 .
$$

Volviendo a la variable original $r$ en $(3.63)\left(d r=e^{k(\rho)} d \rho\right)$ se obtiene

$$
\left[\frac{d^{2}}{d r^{2}}+\tilde{\omega}^{2}\right] \delta x_{1}(r)=0, \quad r \gg 1 .
$$

cuya solución son ondas planas $e^{ \pm i \tilde{\omega} r}$ para $\bar{\omega}^{2}>0$ y exponenciales reales $e^{ \pm \tilde{\omega} r}$ para $\bar{\omega}^{2}<0$. Una palabra de precaución, como se discutió en la sección 3.2.5 la teoría de gauge que vive sobre la brana debe localizarse a una distancia finita $\rho_{\infty}$, luego, para (3.88) definida sobre $\rho_{0} \leq \rho \leq \rho_{\infty}$ existirán autovalores positivos. En el caso $\bar{\omega}^{2}<0$ existe la posibilidad de obtener autovalores negativos, y de echo se encuentran soluciones normalizables con modos negativos precisamente para las región en la que la solución clásica no cumple con las condiciones de convexidad (3.17). En las figuras 3.22 y 3.23 se grafican los autovalores mínimos que permiten soluciones normalizables obtenidos numéricamente como función de $r_{0}$. Se encuentra un acuerdo completo con la figura 3.14: no se encuentran inestabilidades para la solución clásica que satisface $L^{\prime}\left(r_{0}\right)<0$, por otro lado, se encuentran modos negativos (inestables) para la rama derecha (soluciones con $L^{\prime}\left(r_{0}\right)>0$ ) en la figura 3.14. Estos resultados son gratificantes debido a que los modos inestables se encuentran precisamente para las soluciones clásicas de cuerda que no satisfacen las condiciones (3.17). En la sección siguiente voy a resumir estos resultado transformando las ecuaciones de movimiento en ecuaciones del tipo Schrödinger. 


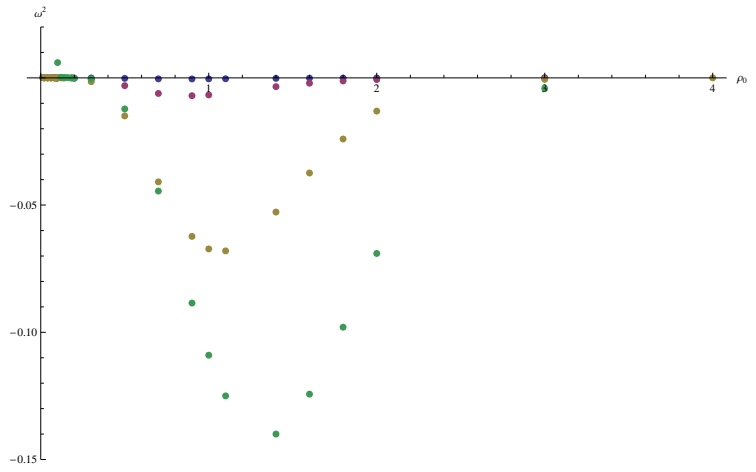

Figura 3.22: Autovalores más bajos $\omega^{2}$ de (3.88) que conducen a soluciones normalizables como función de $\rho_{0}$. Modos negativos (inestables) se encuentran precisamente para las soluciones clásicas que satisfacen $L^{\prime}\left(\rho_{0}\right)>$ 0. Colores como en la fig. 3.14.

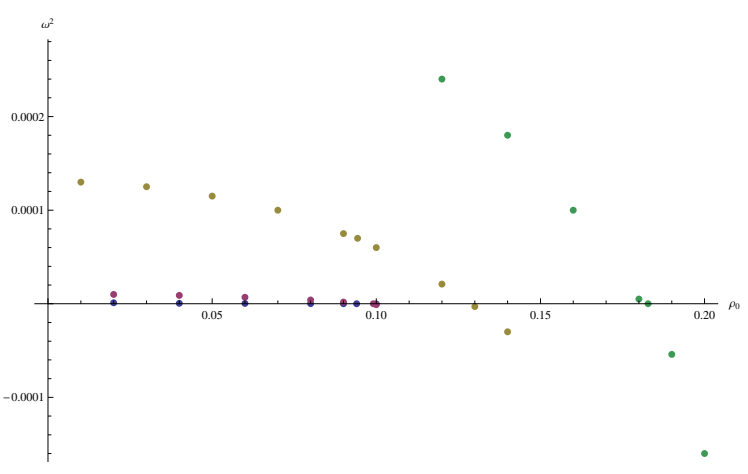

Figura 3.23: Ampliación de la figura 3.22 cerca del origen. Los autovalores más bajos son positivos para las soluciones que satisfacen $L^{\prime}\left(\rho_{0}\right)<0$. Aparece un modo cero precisamente para el valor critico de la función $L\left(\rho_{0}\right)$ (ver fig. 3.14).

\subsection{Análisis de Potenciales de Schrödinger}

En esta sección se analizarán la ecuación de movimiento para la fluctuación (3.22) llevándola a una expresión de tipo Schrödinger (ver apéndice A.1). A partir de la forma del potencial es posible mostrar la existencia, o no, de autovalores negativos y por lo tanto probar la estabilidad de la configuración de cuerda correspondiente.

\subsection{1. $A d S_{5}$}

El potencial de Schrödinger (A.4) para la ecuación (3.77) toma la forma [99]

$$
V(\rho)=2 \frac{\rho^{4}-1}{\rho^{2}}, \quad \rho \in[1, \infty),
$$

aquí $\rho$ debe ser entendido como $\rho=\rho(y)$. El cambio de variables (A.2) que nos conduce a la ecuación de Schrodinger (A.3) puede ser calculado analíticamente

$$
y(\rho)=y_{0}-\frac{1}{4} \mathrm{~B}\left(\frac{1}{\rho^{4}} ; \frac{1}{4}, \frac{1}{2}\right),
$$

con $y_{0}=\frac{\Gamma\left[\frac{1}{4}\right]^{2}}{4 \sqrt{2 \pi}}$. La semi-recta $\rho \in[1, \infty)$ del problema original de Sturm-Liouville, ante el cambio de variables (3.92), se mapea en el intervalo infinito $y \in\left[0, y_{0}\right]$. El potencial (3.91) es divergente en $y_{0}$. Se ha definido un problema de Schrodinger sobre un intervalo finito con condiciones de borde canónicas (ver (A.5)-(A.6)) y por lo tanto, dado que el potencial (3.91) es definido positivo, se obtendrá un espectro discreto. Argumentos estándar de mecánica cuántica permiten afirmar que no existirán soluciones con autovalores negativos. Se concluye que el fondo de $A d S$ dado por (3.34) es estable ante perturbaciones lineales. La figura 3.24 muestra el potencial (3.91) como función 


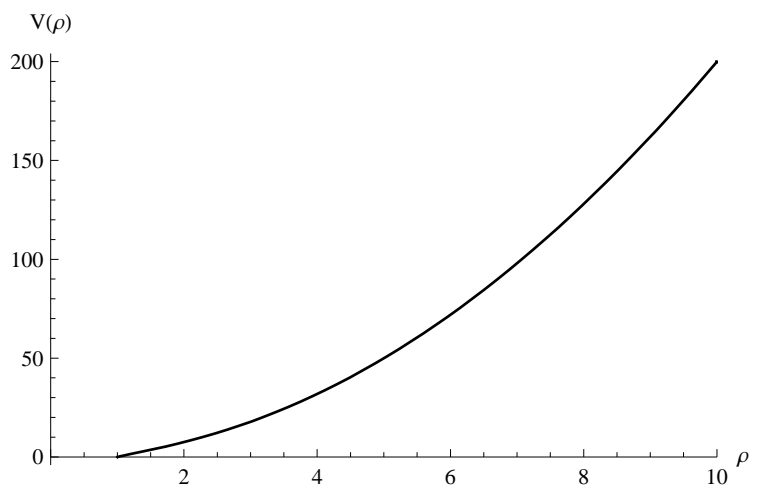

Figura 3.24: Potencial de Schrodinger (3.91) para fluctuaciones en $A d S$. Al ser definido positivo se garantiza la ausencia de modos negativos.

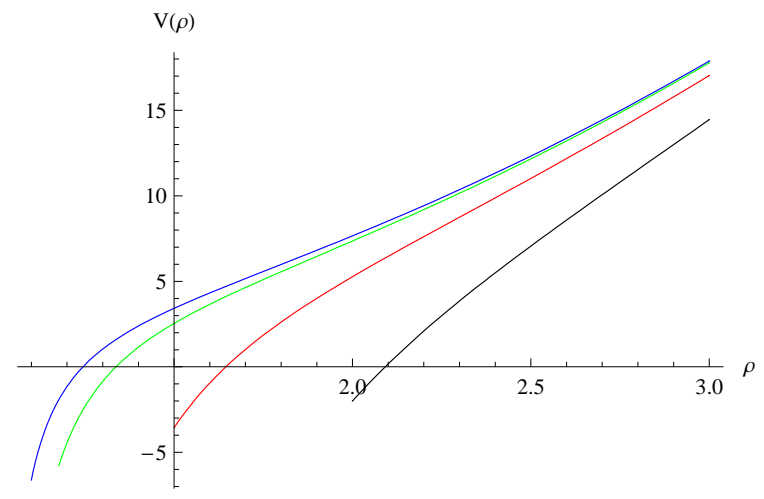

Figura 3.25: $V\left(\rho, \rho_{0}\right)$ (ec.(3.93)) para diferentes valores de $\rho_{0}$. La línea azul corresponde a $\rho_{0}=1,1$, la verde al valor crítico $\rho_{0}=1,177$ y las líneas roja y negra a $\rho_{0}=1,2$ respectivamente. La región en donde el potencial es negativo disminuye a medida que $\rho_{0}$ aumenta. Los autovalores negativos dejan de existir para $\rho_{0} \geq 1,177$.

de $\rho$, la verdadera variable del problema de Schrodinger es $y$ dada por (3.92), la cual es solo un reescaleo del eje horizontal de la fig. 3.24 mapeando $\rho=\infty$ a una distancia finita.

\subsection{2. $A d S_{5}$-Schwarzschild}

El potencial de Schrödinger (A.4) para la ecuación (3.80) toma la forma [99]

$$
V\left(\rho, \rho_{0}\right)=2 \frac{\rho^{8}\left(\rho^{4}-\rho_{0}^{4}\right)-\rho_{0}^{4}\left(4 \rho^{4}-1\right)-3 \rho^{4}}{\rho^{6}\left(\rho^{4}-1\right)}, \quad 1<\rho_{0} \leq \rho<\infty .
$$

El comportamiento de este potencial para diferentes valores de $r_{0}$ a sido graficado en la figura 3.25.

A diferencia del caso de $A d S_{5} \times S^{5}$, existen regiones en donde el potencial toma valores negativos, lo cual esta en acuerdo con los resultados de la sección 3.3.2 en donde se habían encontrado autovalores negativos. El potencial comienza tomando un valor negativo en $\rho_{0}$ dado por $V\left(\rho_{0}, \rho_{0}\right)=-8 / \rho_{0}^{2}$. A medida que $\rho_{0}$ se incrementa la región negativa disminuye y los modos negativos dejan de existir en el valor crítico, encontrado numéricamente en la sección 3.3.2, $\rho_{0 c} \simeq 1,177$ el cual precisamente coincide con el valor crítico de la función longitud $L\left(\rho_{0}\right)$. Se concluye que la solución clásica que satisface $L^{\prime}\left(\rho_{0}\right)>0$ es inestable ante perturbaciones lineales (ver también [99] para un análisis perturbativo de los autovalores). La solución inestable $L^{\prime}\left(\rho_{0}\right)>0$ tiene energía finita $E_{q \bar{q}}$ positiva (ver fig. 3.6), debido a que la configuración de referencia satisface las mismas condiciones de borde, el candidato natural para el proceso de decaimiento es el estado de referencia (quarks libres).

Por completitud se mencionará que debido a que el desarrollo asintótico en esta caso coincide con el de el ejemplo previo, la ecuación de Schrödinger para las fluctuaciones queda definida en un 
intervalo finito. El espectro, por lo tanto, es discreto.

\subsubsection{Maldacena-Núñez}

El potencial de Schrödinger para (3.81) toma la forma

$$
V\left(r, r_{0}\right)=\frac{e^{-2 \phi(r)}}{4}\left(\left(e^{2 \phi(r)}-3 e^{2 \phi\left(r_{0}\right)}\right) \phi^{\prime 2}(r)+2\left(e^{2 \phi(r)}+e^{2 \phi\left(r_{0}\right)}\right) \phi^{\prime \prime}(r)\right), \quad 0<r_{0} \leq r<\infty .
$$

Como antes, $r$ debe ser entendida como $r=r(y)$ y al contrario que los últimos dos casos con el cambio de variables (A.2) se obtiene un problema de Schrödinger en la coordenada $y$ definida sobre la semi-recta $y \in[0, \infty)$. Las figuras 3.26 y 3.27 muestran el potencial de Schrödinger (3.94) para diferentes valores de $r_{0}$. Se deben confrontar estas figuras con los resultados obtenidos en la sección 3.3.3. Lo concluido en esa sección fue que, para todo valor de $r_{0}$, existe un espectro continuo para $\omega^{2}>0$ y numéricamente no se encontraron modos normalizables negativos. En primer lugar, analizemos el espectro continuo para $\omega^{2}>0$. La Figura 3.26 muestra que el potencial es definido positivo para $r_{0} \leq 1,1605$ y asintóticamente toma el valor $V_{\infty}=\frac{1}{4}$. Luego, se podría concluir que no existen soluciones para $0<\bar{\omega}^{2}<V_{\min }$ y que se encuentra un espectro discreto para $V_{\min }<\bar{\omega}^{2}<\frac{1}{4}$ (en caso de ser posible) y un espectro continuo, pero no normalizable, para $\bar{\omega}^{2}>\frac{1}{4}$, todo en contradicción con los resultados mencionados. La concordancia se obtiene cuando se tiene en cuenta el factor $(P Q)^{-\frac{1}{4}}$ que relaciona la solución de la ecuación de Schrödinger $\Psi$ con la fluctuación $\delta x_{1}$ (ver apéndice A.1 eqn. (A.2))

$$
\delta x_{1}=\frac{e^{\frac{\phi(r)}{2}}}{\left(e^{2 \phi(r)}-e^{2 \phi\left(r_{0}\right)}\right)^{\frac{1}{2}}} \Psi \simeq e^{-\frac{r}{2}} \Psi, \quad r \rightarrow \infty .
$$

El factor $e^{-\frac{r}{2}}$ hace que todas las soluciones con $\bar{\omega}^{2}>0$ del problema de Schrödinger satisfagan $\left.\delta x_{1}\right|_{r=\infty}=0$ independientemente de si $\Psi(y)$ es normalizable o no (asintóticamente se tiene $y \simeq r$ ). Sin embargo, para soluciones con $\bar{\omega}^{2}<0$ el factor no es suficiente para hacer que las soluciones (divergentes) satisfagan las condiciones de borde. Se concluye que para todo valor de $r_{0}$ se tiene un espectro continuo para $\bar{\omega}^{2}>0$.

El segundo punto a tener en cuenta es la posibilidad de encontrar estados ligados para $-\frac{1}{2}<$ $\bar{\omega}^{2}<0$ en el límite de $r_{0}$ grande. Como se ha visto en la figura 3.27 , asintóticamente, el potencial comienza tomando el valor $V\left(r_{0}\right) \simeq-\frac{1}{2}$ y en la aproximación lineal se obtiene $V(r) \simeq-\frac{1}{2}+\frac{3}{2}\left(r-r_{0}\right)$. La relación entre las coordenadas $r$ e $y$ (A.2) en el mismo límite es $\left(r-r_{0}\right) \simeq y^{2} / 2$. Todo esto conduce a un oscilador armónico en las coordenadas y con energía de ligadura positiva. Se concluye, por lo tanto, que no existen estados ligados y se encuentra una perfecta concordancia entre el análisis de Schrödinger y los resultados numéricos de la sección 3.3.3.

El resultado es atractivo debido a que si se hubiesen encontrado inestabilidades, no hay candidato disponible para el decaimiento (confrontar con sección 3.4.5). 


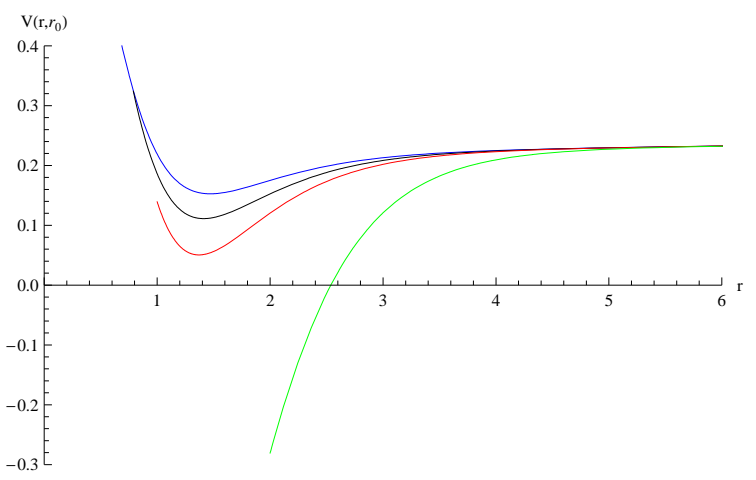

Figura 3.26: Potencial de Schrödinger (3.94). Las líneas azul, negra, roja y verde corresponden con $r_{0}=0,2,0,7,1,2$. el mínimo de potencial se hace negativo para $r_{0} \geq 1,1605$.

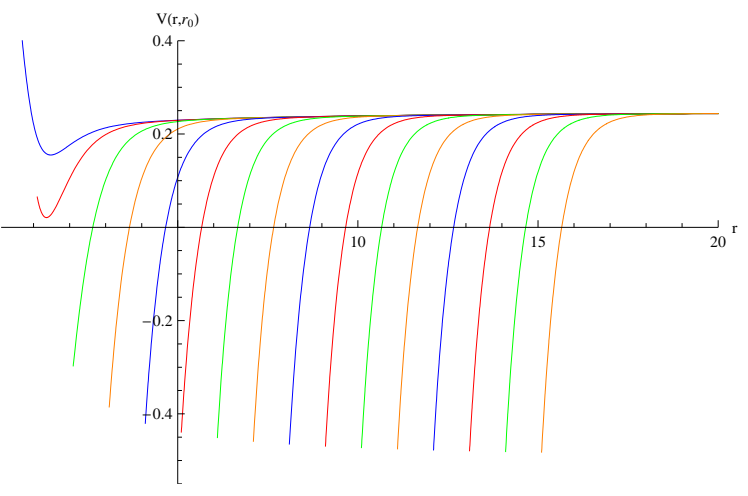

Figura 3.27: Potencial (3.94) como función de $r_{0}$. El potencial asintóticamente tiende a $V_{\infty}=\frac{1}{4}$ en concordancia con (3.83). Para $r_{0}<1,1605$ es positivo y para $r_{0} \geq 1,1605$ contiene regiones negativas. Cerca del tip el potencial puede aproximarse asintóticamente (para $r_{0}$ grande) por $V(r) \simeq-\frac{1}{2}+\frac{3}{2}\left(r-r_{0}\right)$

\subsubsection{Klebanov-Strassler}

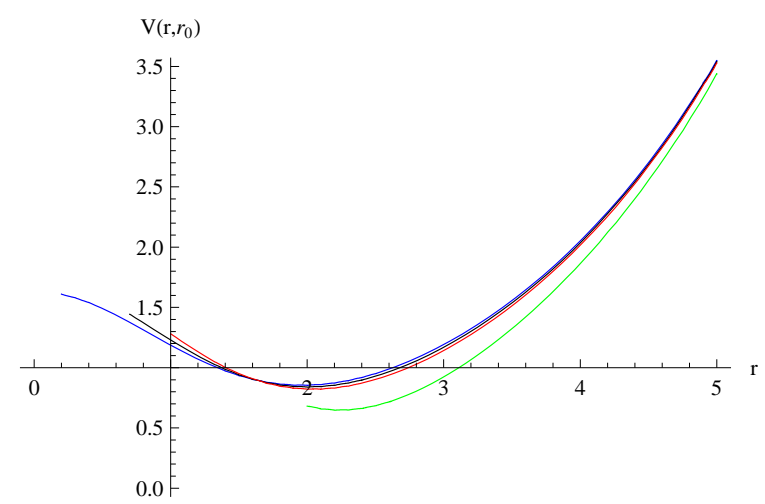

Figura 3.28: Potencial de Schrodinger (3.96). Las líneas azul, negra, roja y verde corresponden a $r_{0}=0,2,0,7,1,2$. El potencial es definido positivo y por lo tanto se obtienen autovalores positivos.

El potencial para la fluctuación en el caso de Klebanov-Strassler (3.84) es

$$
\begin{aligned}
V\left(r, r_{0}\right)= & -\frac{3 K(r)}{8 h^{3}(r) h\left(r_{0}\right)}\left[4 h(r)\left(h(r)+h\left(r_{0}\right)\right) h^{\prime}(r) k^{\prime}(r)\right. \\
& \left.\left.-k(r)\left(3 h(r)+7 h\left(r_{0}\right)\right) h^{\prime 2}(r)+4 h(r)\left(h(r)+h\left(r_{0}\right)\right) h^{\prime \prime}(r)\right)\right]
\end{aligned}
$$

El comportamiento asintótico de las funciones $P, Q$ en este caso conduce a un problema de Schrödinger definido en un intervalo finito en la coordenada $y$ (ver apéndice A.1). La figura (3.28) muestra la forma del potencial para varios valores de $r_{0}$. El potencial es definido positivo y, por lo tanto, no existen soluciones con $\omega^{2}<0$. El intervalo finito en el cual el problema de Schrödinger esta definido 


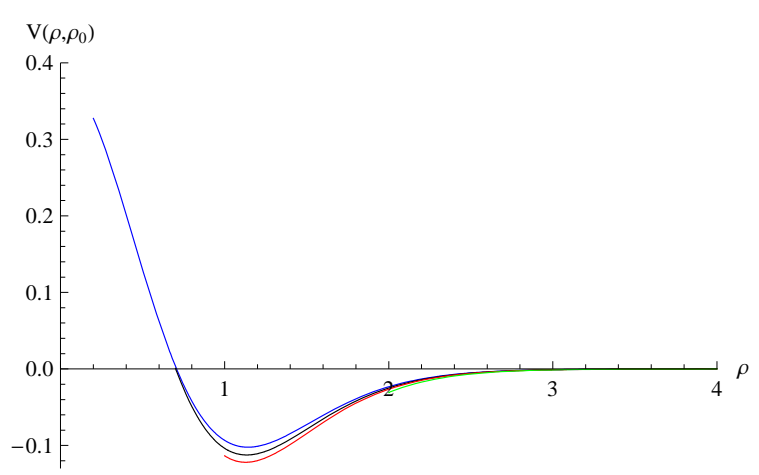

Figura 3.29: Potencial de Schrödinger (3.97) para diferentes valores de $\rho_{0}$ y $\mu=-1$. Las líneas azul, negra, roja y verde se corresponden con $\rho_{0}=0,2,0,7,1,2$ respectivamente.

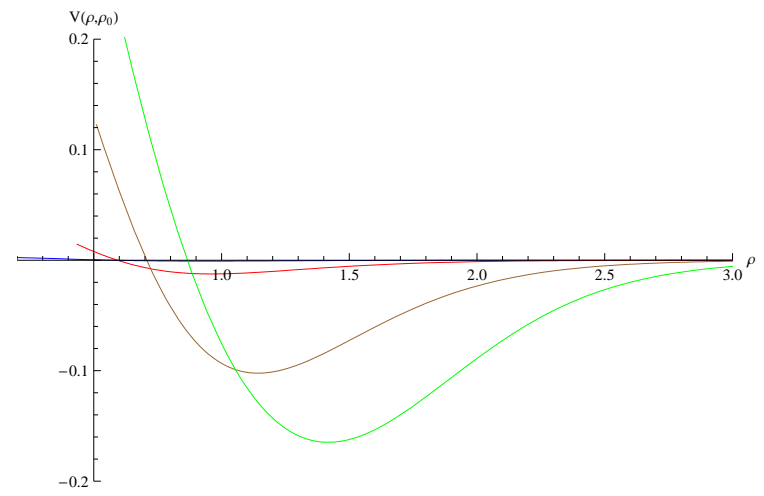

Figura 3.30: Potencial de Schrödinger para $\rho_{0}=0,2$ y para diferentes valores del parámetro $\mu$. Las curvas azul, roja, marrón $\mathrm{y}$ verde se corresponden con $\mu=$ $-1,8,-1,5,-1,-0,8$.

implica un conjunto discreto de autovalores. Se encuentra perfecta concordancia con los resultados de la sección 3.3.4.

\subsubsection{Maldacena-Núñez Generalizado}

El potencial de Schrödinger para fluctuaciones en el caso generalizado de Maldacena-Nuñez puede escribirse como

$$
V\left(\rho, \rho_{0}\right)=\frac{2}{e^{8 f(\rho)+2 k(\rho)}}\left(2\left(e^{8 f(\rho)}-e^{8 f\left(\rho_{0}\right)}\right) f^{\prime 2}(\rho)+\left(e^{8 f(\rho)}+e^{8 f\left(\rho_{0}\right)}\right)\left(f^{\prime \prime}(\rho)-k^{\prime}(\rho) f^{\prime}(\rho)\right)\right)
$$

El comportamiento asintótico de las funciones $P, Q$ (ver apéndice A.1 y la ec. (3.89)) conduce a un problema de Schrödinger formulado en la semi-recta $y \in[0, \infty)$. La figura (3.29) muestra el comportamiento del potencial para diferentes valores de $r_{0}$ y $\mu=-1$. El potencial se hace negativo por sobre el valor crítico $\rho_{*}$ y asintóticamente $V_{\infty}=0$ en concordancia con (3.89) y con la existencia de modos negativos encontrados numéricamente en la sección 3.3.5. El factor $(P Q)^{-\frac{1}{4}}$ que relaciona la función de onda de Schrödinger $\Psi$ con la función $\delta x_{1}$ tiende a una constante en infinito, lo cual no cambia el desarrollo asintótico de las soluciones $\Psi$ (confrontar con la sección 3.4.3). La figura 3.30 muestra el potencial de Schrödinger para un valor fijo $\rho_{0}=0,2$ y para diferentes valores de $\mu$. El mínimo de potencial disminuye a medida que $\mu$ se acerca al valor $-\frac{2}{3}$. Como se ha mencionado, la solución original de Maldacena-Nuñez (3.47) no se conecta continuamente con la clase generalizada de soluciones (3.63). Se encontró perfecta concordancia con los resultados numéricos de la sección 3.4.5 pero no es claro cuál es el estado final de decaimiento. 


\subsection{Lazo de 't Hooft}

El dual electromagnético de las líneas de Wilson en teorías de Yang-Mills son las líneas de 't Hooft [124]. En cuatro dimensiones, se supone que el mecanismo para confinamiento se debe a la condensación de monopolos magnéticos (efecto Meissner dual), el análisis en [124] concluye que se debería observar un potencial apantallado entre un par $m \bar{m}$ de monopolos cuando se observa confinamiento entre el par $q \bar{q}$. El confinamiento oblicuo es una generalización de esta idea en la cual se estudia el confinamiento de diones.

La prescripción dada por la conjetura para calcular lazos de 't Hooft en las soluciones de MN y KS ha sido propuesta en los mismos trabajos [6]-[7] (ver [125] y también [118], en donde se analiza una cuestión técnica, corrigiendo el 2-ciclo propuesto en [6]) y consiste en enroscar una D3-brana de prueba en el mismo 2-ciclo sobre el cual están enroscadas las $D 5$-branas que generan la geometría (ver también [92]). El resultado de esta construcción es una D1-brana efectiva (cuerda) la cual se analiza en completa analogía a la cuerda fundamental que ha sido discutida en secciones previas. La diferencia con respecto al lazo de Wilson es que el lazo de 't Hooft en teorías genéricas que no son duales ante simetría $\mathrm{S}$ es sensible a la variedad interna de 5 dimensiones.

\subsubsection{Maldacena-Núñez}

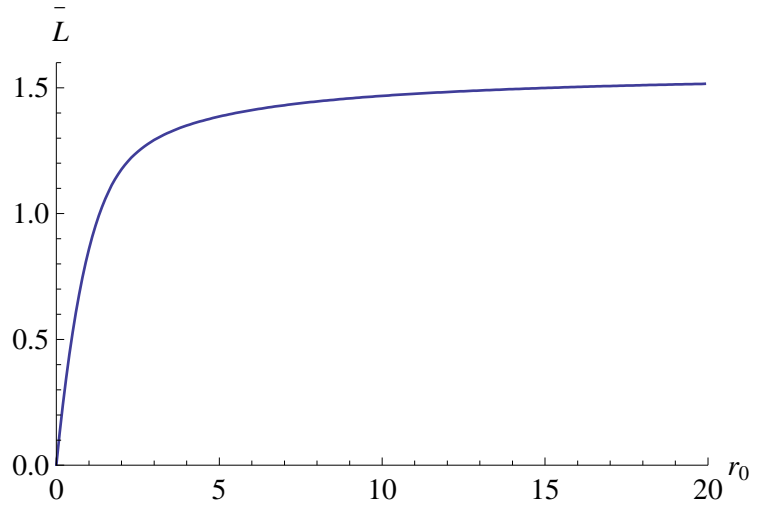

Figura 3.31: Función longitud para la cuerda efectiva como función de $r_{0}$ para el lazo de Wilson en el caso de Maldacena-Núñez.

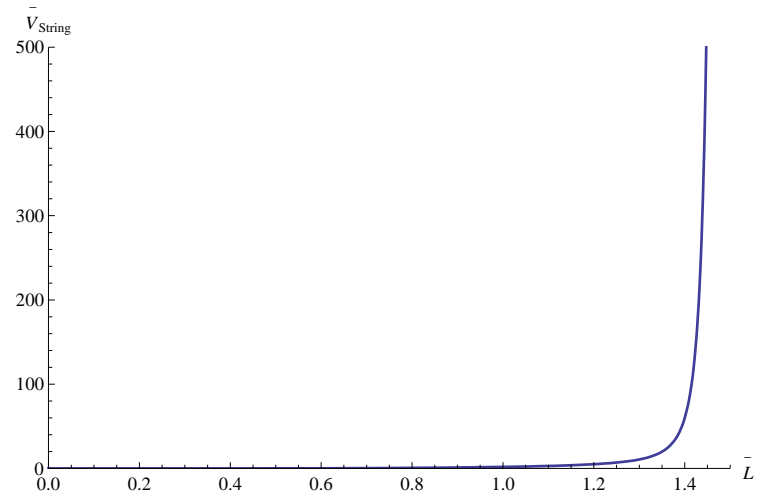

Figura 3.32: Energía del par monopoloantimonopolo como función de la longitud de separación.

La variedad en la que se embebe la $D 3$ en la métrica $(3.47)$ es $[125]^{10}$

$$
\mathcal{M}_{4}=[t, x, r(x), \theta=\tilde{\theta}, \varphi=2 \pi-\tilde{\varphi}, \psi=\pi] .
$$

\footnotetext{
${ }^{10}$ Las coordenadas restantes se fijan a constantes. El valor de la coordenada $\psi$ se fija pidiendo que la $S^{2}$ tenga volumen mínimo.
} 
La métrica inducida sobre $\mathcal{M}_{4}$ es

$$
d s_{i n d}^{2}=\alpha^{\prime} N e^{\phi}\left[-d t^{2}+\left(1+\dot{r}^{2}\right) d x^{2}+\left(e^{2 h}+\frac{1}{4}(1-a)^{2}\right)\left(d \theta^{2}+\sin ^{2} \theta d \varphi^{2}\right)\right],
$$

y de las expresiones (3.49) para $a, h$ queda

$$
V_{S^{2}}(r) \equiv \frac{1}{4}(1-a(r))^{2}+e^{2 h(r)}=r \tanh r .
$$

Nótese que la $S^{2}$ colapsa suavemente en el origen. Integrando la acción DBI ${ }^{11}$

$$
S_{D B I}=-T_{D 3} \int d^{4} \sigma e^{-\phi} \sqrt{g_{\text {ind }}}
$$

sobre la variedad interna $\left(S_{\theta \varphi}^{2}\right)$ y la coordenada temporal se tiene

$$
S_{e f f}=4 \pi T_{D 3} \mathcal{T}\left(\alpha^{\prime} N\right)^{2} \int e^{\phi} r \tanh r \sqrt{1+\dot{r}^{2}} d x .
$$

La diferencia importante con respecto al cálculo del lazo de Wilson en el fondo de MN (ver sección 3.2.3) reside en las funciones $f(r)$ y $g(r)$ que en (3.50)-(3.51) están multiplicadas por el volumen de la 2-esfera (3.100).

En las figuras 3.31 se gráfica el comportamiento de la función longitud (3.9) como función de $r_{0}$. La función longitud es una función creciente en $r_{0}$ y a partir de las discusiones previas se espera que las soluciones sean inestables. Puede verse la inestabilidad de la forma de embeber la D1 efectiva en el caso de MN debido a que una fluctuación a lo largo de la dirección $x_{1}$ dependiente solamente de $t, r$ está desacoplada de las fluctuaciones angulares (fluctuación consistente). La ecuación de movimiento para $\delta x_{1}$ es $(3.22)$ con $f(r)=g(r)=h(r)=r \tanh r e^{\phi(r)}$. El comportamiento asintótico de la fluctuación es el mismo que en el caso del lazo de Wilson, sin embargo, este comportamiento cambia drásticamente cerca del origen debido a que $f(r)$ tiende a cero. Como se ve en la figura 3.33 existen autovalores negativos para todo valor de $r_{0}$. En la figura 3.34 se muestra el comportamiento del potencial de Schrödinger asociado con la ecuación de movimiento para la fluctuación.

En la figura 3.32 se gráfica la energía como función de la longitud de separación $L$. La energía de la configuración es positiva para todo $L$, este echo y la inestabilidad de la configuración sugiere que la configuración estable para dadas condiciones de borde, es la correspondiente a dos " líneas rectas ". Contrariamente al caso del lazo de Wilson, las " líneas rectas" (usadas como estado de referencia para regularizar la energía) pueden terminar en el origen debido a que estas corresponden a $D 3$ enroscadas en una $S^{2}$ topológica de (3.47) que colapsa suavemente en el origen. 

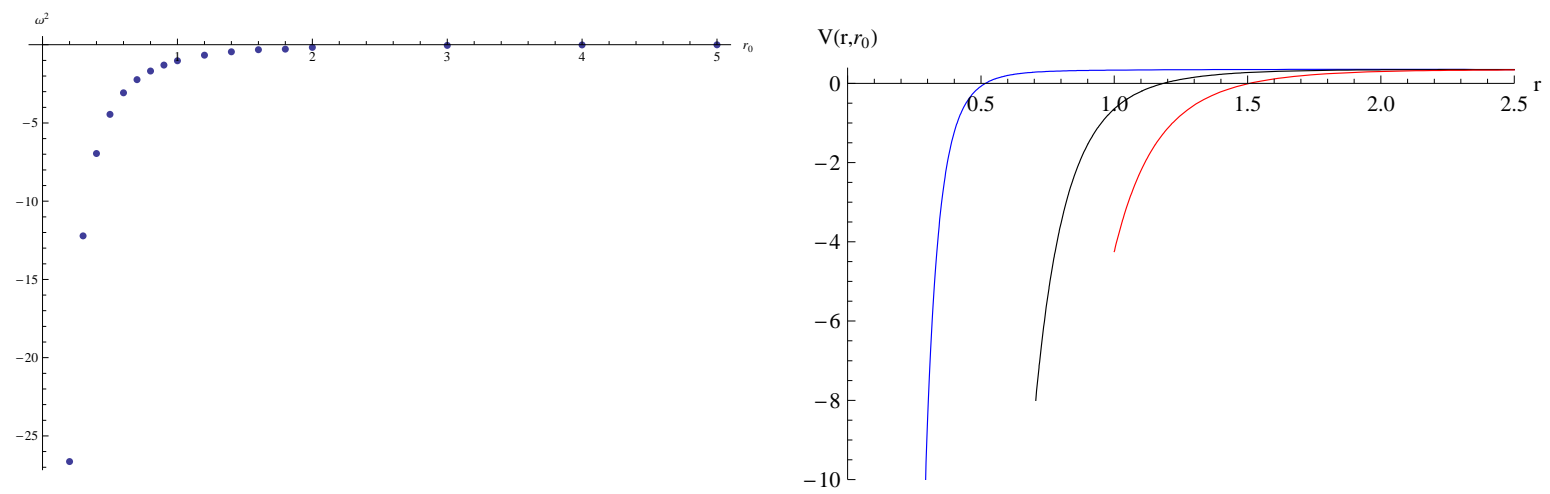

Figura 3.33: Autovalores más bajos $\omega^{2}$ para fluctuaciones que solo dependen de las coordenadas $t, r$ como función de $r_{0}$ en el caso del lazo de 't Hooft para el fondo de MaldacenaNúñez.

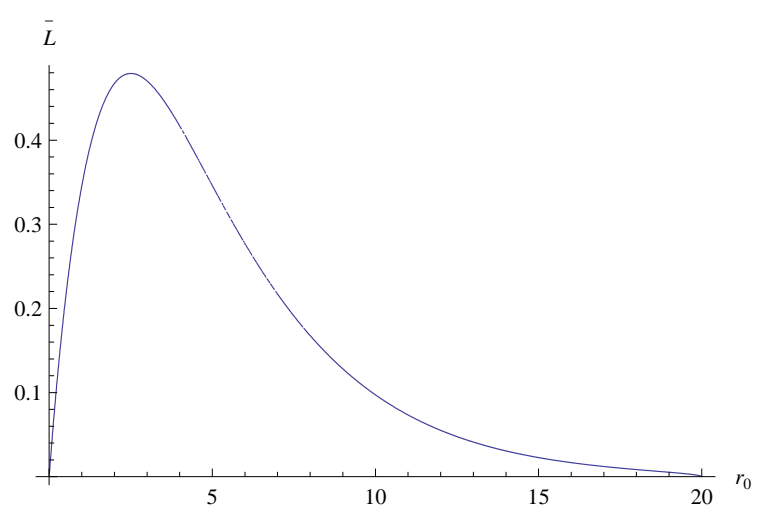

Figura 3.34: Potencial de Schrodinger para fluctuaciones que dependen de las coordenadas $t, r$ como función de $r_{0}$. Las curvas azul, negra y roja corresponden a $r_{0}=0,2,0,7,1$.

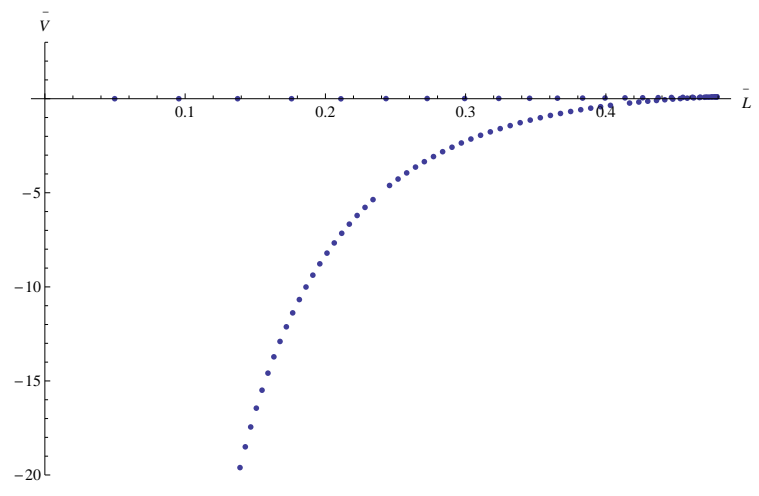

Figura 3.36: La energía del par monopoloantimonopolo como función de la longitud de separación.

\subsubsection{Klebanov-Strassler}

Este caso de nuevo involucra enroscar una $D 3$ sobre una $S^{2}$ topológica dentro de (3.54) (para su parametrización ver el apéndice A de [126]). Hay diferencias importantes con respecto al caso de MN, en esta caso la $H_{3}$ que soporta la geometría de KS contribuye a la acción de la cuerda (3.1), y además conduce a un entrelazamiento entre las fluctuaciones de las coordenadas angulares y las del plano. El comportamiento diferente en el UV con respecto al caso de MN es la razón por la cual la función $L\left(r_{0}\right)$ tiene, a priori, regímenes estables (ver figura 3.35). El comportamiento de $\bar{V}(\bar{L})$ muestra que el potencial esta apantallado para valores grandes de $L$ lo cual concuerda con el potencial lineal para el caso del lazo de Wilson (ver [92] para estudiar un ejemplo relacionado).

\footnotetext{
${ }^{11}$ Usando el ansatz para el fijado de gauge (3.98) en la acción (3.101) se obtiene la ecuación de movimiento correcta para $r(x)$ lo cual coincide con (3.7).
} 
El análisis del presente caso es análogo al realizado en la sección 3.2.2, en resumen, cuando la configuración de energía se hace positiva, la solución de dos líneas rectas es favorecida. Como para el caso de $\mathrm{MN}$ esto es posible sin un horizonte en este caso debido a que la D3 esta enroscada sobre una $S^{2}$ que colapsa suavemente en el origen. No se realizó el análisis de las ecuaciones de movimiento para las fluctuaciones acopladas para chequear las inestabilidades de la rama izquierda de la fig. 3.35 .

\subsection{Fluctuaciones del Lazo de Wilson en representaciones de orden más alto}

En esta sección se estudiarán las fluctuaciones bosónicas y fermiónicas de la configuración de branas dual a un lazo de Wilson en la representación simétrica mencionada en la sección 1.1.3.

En particular se analizarán las fluctuaciones correspondientes a un lazo de Wilson- 't Hooft supersimétrico [127, 128], para un camino $\mathcal{C}$ recto en la representación simétrica del grupo. A partir de este operador se puede obtener el resultado tanto para el lazo de Wilson como para el de 't Hooft.

\subsubsection{Acción general para D-branas}

\section{Acción Bosónica}

La parte bosónica de la acción de un Dp-brana, llamada acción de Dirac-Born-Infeld se escribe

$$
S_{B}=-T_{D p} \int d^{p+1} \sigma e^{-\varphi} \sqrt{-\operatorname{det}\left(g+2 \pi \alpha^{\prime} F\right)}+T_{D p} \int P\left[C_{p+1}\right]
$$

en donde $T_{D p}=(2 \pi)^{-p} \alpha^{-\frac{p+1}{2}}$ es la tensión de la brana, $F$ es el campo de gauge que vive en su volumen de mundo, $\varphi$ es el dilatón y $g$ y $P\left[C_{p+1}\right]$ son el pullback de la métrica y de la $p+1$ forma a la cual se acopla respectivamente. El segundo término de la acción es el denominado término de Wess-Zumino-Witten y es responsable de la existencia de gravitones gigantes [129, 130] y el efecto Myers [131].

Físicamente la expresión (3.103) es la acción efectiva de baja energía para los modos no masivos de una brana con campos de fondo $\left(g_{\mu \nu}, \varphi\right.$ y formas de Ramond-Ramond $\left.C_{p+1}\right)$ [132, 133]. En el caso del lazo de Wilson en la representación simétrica, que corresponde a una $D 3$-brana, solo tendremos acoplamiento al $C_{4}$ de fondo.

\section{Acción Fermiónica}

La acción fermiónica cuadrática para nuestro caso (D3-brana en un fondo $\left.\mathrm{AdS}_{5} \times S^{5}\right)$ toma la forma [136] 


$$
S_{D p}^{(F)}=\frac{T_{D p}}{2} \int d^{p+1} \sigma \sqrt{-\operatorname{Det}(M)} \bar{\Theta}\left(1-\Gamma_{D p}\right)\left(\tilde{M}^{-1}\right)^{\alpha \beta} \Gamma_{\beta} D_{\alpha} \Theta,
$$

en donde $M=g+F$. El campo fermiónico $\Theta$ es un fermion de Weyl con quiralidad positiva en $d=9+1$, esto significa que posee 32 componentes reales y es reducible frente a $S O(9,1)$ a 2 fermiones MW. En lo que sigue consideraremos a $\Theta$ como un fermion de 64 componentes, las partes superiores e inferiores corresponden a los dos fermiones MW, las matrices $\Gamma$ se representaran entonces como matrices de $64 \times 64$ dimensiones. La derivada covariante $D_{\alpha}$ se escribe

$$
D_{\alpha}=\left(\partial_{\alpha} x^{m}\right)\left(\nabla_{m}+\frac{1}{16 \cdot 5 !} F_{n p q r t} \Gamma^{n p q r t}\left(i \sigma_{2}\right) \Gamma_{m}\right), \quad \nabla_{m}=\partial_{m}+\frac{1}{4} \omega \frac{n p}{m} \Gamma_{\underline{n p}} .
$$

Letras griegas denotan índices sobre el volumen de mundo de la brana, letras latinas corresponden a coordenadas del espacio 10-dimensional y los indices subrayados corresponden a coordenadas del espacio tangente (planos). $\tilde{M}_{\alpha \beta}=g_{\alpha \beta}+\tilde{\Gamma} F_{\alpha \beta}$ con $\tilde{\Gamma}=\gamma^{11} \otimes \sigma_{3}{ }^{12}$. La matriz $\Gamma_{D p}$ en (3.104) es

$$
\begin{aligned}
\Gamma_{D p} & =\frac{\sqrt{-\operatorname{Det}(g)}}{\sqrt{-\operatorname{Det}(g+F)}} \Gamma_{D p}^{(0)} \otimes\left(\sigma_{3}\right)^{\frac{p+1}{2}}\left(-i \sigma_{2}\right) \sum_{q} \Gamma^{\alpha_{1} \ldots \alpha_{2 q}} F_{\alpha_{1} \alpha_{2}} \ldots F_{\alpha_{2 q-1} \alpha_{2 q}} \otimes \frac{\sigma_{3}^{q}}{2^{q} q !} \\
\Gamma_{D p}^{(0)} & =\frac{\epsilon^{\alpha_{1} \ldots \alpha_{p+1}}}{(p+1) ! \sqrt{-\operatorname{Det}(g)}} \Gamma_{\alpha_{1} \ldots \alpha_{p+1}}
\end{aligned}
$$

La acción (3.104) más la parte bosónica (3.103) preserva la simetría $\kappa$ y proyecta al volumen de mundo la simetría del target. Es un generalización de la acción de Green y Schwarz para la cuerda $[134,135]$.

\subsubsection{Lazo de Wilson-'t Hooft y sus fluctuaciones}

El valor de expectación del lazo de Wilson en la representación simétrica puede obtenerse en la teoría dual gravitatoria estudiando soluciones de D3-branas enroscadas de forma tal que su volumen de mundo sea $\mathrm{AdS}_{2} \times S^{2} \subset \mathrm{AdS}_{5}$, con campo eléctrico encendido y ubicadas en un punto de $S^{5}[51]$.

\section{Solución}

El fondo en el que embebemos la brana tiene encendidos $g_{\mu \nu}, F_{5}$ y $\varphi$. El dilaton (que aparece en (3.103)) es constante para el fondo AdS, y ya fue factorizado en la definición de $T_{p}$. Escribimos la geometría $\mathrm{AdS}_{5} \times S^{5}$ como

$$
d s^{2}=L^{2}\left(\frac{\cosh ^{2}(u)}{r^{2}}\left(-d t^{2}+d r^{2}\right)+d u^{2}+\sinh ^{2}(u)\left(d \theta^{2}+\sin ^{2} \theta d \phi^{2}\right)\right)+L^{2}\left(d \alpha_{1}^{2}+\sin ^{2} \alpha_{1} d \Omega_{4}^{2}\right) .
$$

${ }^{12} \gamma^{11}$ es la matriz quiral $32 \times 32$ en $d=9+1$ 
En estas coordenadas $F_{5}$ toma la forma

$$
F_{5}=4 L^{4} \frac{\sinh ^{2}(u) \cosh ^{2}(u)}{r^{2}} d t \wedge d r \wedge d u \wedge d \theta \wedge d \phi \equiv d C_{4},
$$

y su potencial puede ser escrito como

$$
C_{4}=4 L^{4} \frac{f(u)}{r^{2}} d t \wedge d r \wedge d \theta \wedge d \phi
$$

$\operatorname{con} f(u)=\frac{1}{32} \sinh (4 u)-\frac{u}{8}$.

Agreguemos una D3-brana en esta geometría con un campo electromagnético viviendo en su volumen de mundo. Debemos ahora escoger un ansatz que de cuenta de un lazo recto en la teoría de gauge. La elección apropiada es $X^{\mu}(\sigma)=\left(t, r, u, \theta, \phi, \alpha_{i}\right)=\left(\sigma_{0}, \sigma_{1}, u\left(\sigma_{1}\right), \sigma_{2}, \sigma_{3}\right.$, ctes $\left.\ldots\right)$. Geométricamente esta elección corresponde a enrollar la $D 3$ en la $S^{2}$ parametrizada por $\theta, \phi$ en (3.107) y a extenderla en las coordenadas $t, r$. El ansatz para el campo electromagnético en el volumen de mundo es

$$
F=\frac{q}{r^{2}} d t \wedge d r+k \sin \theta d \theta \wedge d \phi
$$

donde $q$ y $k$ dan cuenta de las cargas eléctricas y magnéticas. Desde el punto de vista dual la solución con ambas cargas encendidas se correlata con el Wilson loop de un dyon (lazo de Wilson-t'Hoooft).

La acción (3.103) queda

$$
S_{B}=-T_{D 3} \int d^{4} \sigma \sqrt{-\operatorname{det}(g+F)}+T_{D 3} \int P\left[C_{4}\right],
$$

en donde sin pérdida de generalidad se utilizarán unidades en las cuales $2 \pi \alpha^{\prime}=1$. Para obtener la solución correspondiente a un lazo de Wilson se debe fijar $k=0$ [137] mientras que para dar cuenta del lazo de 't Hooft se debe tomar $q=0$.

$\mathrm{Al}$ insertar el ansatz en las ecuaciones de movimiento obtenemos que $u=u_{e q}$ es constante y su valor queda fijado por las cargas $(q, k)$ según

$$
q=\operatorname{coth}\left(u_{e q}\right) \sqrt{L^{4} \sinh ^{2}\left(u_{e q}\right)-k^{2}}
$$

El resultado obtenido generaliza el de [137] que se obtiene para $k=0$.

\section{Fluctuaciones}

En esta sección se calcularán las acciones bosónica y fermiónica para pequeñas fluctuaciones alrededor de la solución (3.112). 


\section{- Bosónicas}

El objetivo es expandir la acción (3.111) a segundo orden en fluctuaciones. Las perturbaciones a la solución se escriben

$$
u=u_{e q}+\delta u, \quad \theta^{\hat{i}}=\theta_{0}^{\hat{i}}+\delta \theta^{\hat{i}}, \quad F=\frac{q}{r^{2}} d t \wedge d r+k \sin \theta d \theta \wedge d \phi+f .
$$

En lo que sigue se ignorarán las fluctuaciones $\delta \theta^{\hat{i}}$ debido a que las mismas se desacoplan. La acción bosónica para las fluctuaciones relevantes se puede escribir

$$
\begin{aligned}
S_{B}^{(2)}= & \frac{T_{D 3}}{4} \int d^{4} \sigma\left[\frac { 1 } { r ^ { 2 } } \left(\frac { 1 } { L ^ { 4 } \operatorname { s i n h } ^ { 4 } ( u _ { e q } ) + k ^ { 2 } } \left[2 k r ^ { 2 } \sqrt { \operatorname { s i n h } ^ { 2 } ( u _ { e q } ) L ^ { 4 } - k ^ { 2 } } \left(f_{t \phi} f_{r \theta}-f_{t \theta} f_{r \phi}\right.\right.\right.\right. \\
& \left.+f_{t r} f_{\theta \phi}\right)+L^{4} \cosh \left(u_{e q}\right) \csc (\theta) \sinh ^{3}\left(u_{e q}\right)\left(\left(-f_{\theta \phi}^{2}+r^{2}\left(f_{t \phi}^{2}-f_{r \phi}^{2}\right)\right)\right. \\
& \left.\left.\left.+r^{2} \sin \theta^{2}\left(f_{t \theta}^{2}-f_{r \theta}^{2}+r^{2} f_{t r}^{2}\right)\right)\right]\right) \\
& \left.-\frac{L^{4} \cosh \left(u_{e q}\right) \sinh \left(u_{e q}\right)}{r^{2}}\left(\csc (\theta) \partial_{\phi} \delta u^{2}+\sin (\theta)\left(\left(\partial_{r} \delta u^{2}-\partial_{t} \delta u^{2}\right) r^{2}+\partial_{\theta} \delta u^{2}\right)\right)\right]
\end{aligned}
$$

Nótese que el primer término se anula tanto para el operador de Wilson $(k=0)$ como para el de 't Hooft $(q=0)$ de lo cual se sigue que este es un termino enteramente originado por el campo diónico. Introduciendo la métrica deformada $\hat{g}$ (métrica de cuerda abierta)

$$
\hat{g}_{\alpha \beta}=g_{\alpha \beta}-F_{\alpha \gamma} g^{\gamma \delta} F_{\delta \beta}=\frac{\sinh ^{4}\left(u_{e q}\right) L^{4}+k^{2}}{L^{2} \sinh ^{2}\left(u_{e q}\right)}\left(\begin{array}{cccc}
-\frac{1}{r^{2}} & 0 & 0 & 0 \\
0 & \frac{1}{r^{2}} & 0 & 0 \\
0 & 0 & 1 & 0 \\
0 & 0 & 0 & \sin ^{2} \theta
\end{array}\right),
$$

la ecuación (3.114) toma la forma

$$
\begin{aligned}
S_{B}^{(2)}= & \frac{T_{D 3}}{2}\left(\frac{L^{4} \cosh \left(u_{e q}\right) \sinh ^{3}\left(u_{e q}\right)}{L^{4} \sinh ^{4}\left(u_{e q}\right)+k^{2}}\right) \int d^{4} \sigma\left[\sqrt{-\hat{g}}\left(L^{2} \hat{g}^{\alpha \beta} \partial_{\alpha} \delta u \partial_{\beta} \delta u+\frac{1}{2} \hat{g}^{\alpha \beta} \hat{g}^{\gamma \delta} f_{\alpha \gamma} f_{\beta \delta}\right)\right. \\
& \left.+\frac{k \sqrt{\sinh ^{2}\left(u_{e q}\right) L^{4}-k^{2}}}{L^{4} \cosh \left(u_{e q}\right) \sinh ^{3}\left(u_{e q}\right)}\left(f_{t \phi} f_{r \theta}-f_{t \theta} f_{r \phi}+f_{t r} f_{\theta \phi}\right)\right] \\
= & \frac{T_{D 3}}{2}\left(\frac{L^{4} \cosh \left(u_{e q}\right) \sinh ^{3}\left(u_{e q}\right)}{L^{4} \sinh ^{4}\left(u_{e q}\right)+k^{2}}\right) \int d^{4} \sigma \sqrt{-\hat{g}}\left(L^{2} \hat{g}^{\alpha \beta} \partial_{\alpha} \delta u \partial_{\beta} \delta u+\frac{1}{2} \hat{g}^{\alpha \beta} \hat{g}^{\gamma \delta} f_{\alpha \gamma} f_{\beta \delta}\right. \\
& \left.+\frac{k q}{2 L^{4} \sinh ^{2}\left(2 u_{e q}\right)} \hat{\epsilon}^{\alpha \beta \gamma \delta} f_{\alpha \beta} f_{\gamma \delta}\right),
\end{aligned}
$$

en donde $\hat{\epsilon}^{\alpha \beta \gamma \delta}=\frac{\epsilon^{\alpha \beta \gamma \delta}}{\sqrt{-\hat{g}}}$. Nótese que como consecuencia de lo comentado luego de (3.114) se obtiene un término proporcional a $\tilde{f} f$ cuya constante de proporcionalidad es $\frac{k q}{2 L^{4} \sinh ^{2}\left(2 u_{e q}\right)}$ de lo cual se observa que es una contribución propia de este operador de Wilson-'t Hooft. 


\section{- Fermiónicas}

A continuación escribiremos el resultado para las fluctuaciones de la acción fermiónica para la D3 brana, su derivación se relegará a la sección A.3 del apéndice.

Puesto que en la solución clásica (3.112) los campos fermiónicos están apagados, $\Theta$ en (3.104) representa la fluctuación propiamente dicha, el resultado final es

$$
S_{F}^{(2)}=\frac{T_{D 3}}{2}\left(\frac{L^{4} \cosh \left(u_{e q}\right) \sinh ^{3}\left(u_{e q}\right)}{L^{4} \sinh ^{4}\left(u_{e q}\right)+k^{2}}\right) \int d^{4} \sigma \sqrt{\hat{g}} \bar{\Theta} \hat{\Gamma}^{\alpha} \hat{\nabla}_{\alpha} \Theta
$$

en donde $\hat{g}$ se definió en (3.115) y en esta ultima expresión el espinor $\Theta$ tiene 16 componentes reales debido a que el fijado de gauge de la simetría $\kappa, \tilde{\Gamma} \Theta=\Theta$ anula la mitad de las componentes. Sorprendentemente una series de redefiniciones y rotaciones del campo fermiónico permiten reescribir la acción resultante como una acción para un fermion libre en espacio curvo.

\subsection{Conclusiones}

En el capitulo 3 se ha analizado la propuesta de la conjetura de Maldacena para calcular lazos de Wilson via teoría de cuerdas en fondos gravitatorios y se ha estudiado su estabilidad ante perturbaciones lineales.

La prescripción del lado de cuerdas involucra calcular el área mínima para la hoja de mundo de una cuerda cuyos extremos están sobre el lazo y ubicados en un valor fijo de la coordenada holográfica. Cuando los extremos se ubican en infinito se obtiene un area divergente y es necesario realizar una regularización para obtener un resultado que tenga sentido. En la sección 3.1 se ha resumido esta prescripción y se ha mostrado cómo obtener un resultado finito. Se ha elegido regularizar la acción mediante el procedimiento original propuesto en [45, 46]. Este proceso tiene en cuenta que en el cálculo del área mínima de la acción de Nambu-Goto hay contribuciones de la auto-energía (masa) de los quarks externos. Con esta interpretación se reproducen resultado conocidos para $A d S$ y $A d S$ térmico. De echo, la regularización es responsable de cambiar el área positiva en una energía potencial negativa y atractiva. Cuando se estudian fondos gravitatorios que terminan suavemente ( $A d S$ en coordenadas globales, MN y $\mathrm{KS}$ ) nos encontramos con un rompecabezas debido a que las cuerdas rectas que se extienden a lo largo de la dirección redial usadas para la substracción deben terminar en algún lugar en el volumen del espacio. Se concluye que la interpretación correcta para el proceso de substracción es que se está comparando la hoja de mundo de la cuerda con respecto a un estado de referencia que consiste de la hoja de mundo de dos cuerdas rectas cuyos extremos están en las antípodas de una dirección compacta (representada gráficamente en la fig.3.10). Se dice entonces que el estado de referencia, en general, satisface diferentes condiciones de borde que la hoja de mundo con la que se calcula el valor de expectación 
del lazo de Wilson. Esta última observación es bienvenida para los casos de MN y KS en donde ocurre confinamiento lineal para hojas de mundo que poseen energía regularizada positiva (ver figs. 3.9 and 3.13): si el estado de referencia satisficiera las mismas condiciones de borde que la hoja de mundo del lazo de Wilson, el comportamiento lineal obtenido no debería ser tomado en cuenta debido a que el estado de referencia $\left(E_{q \bar{q}}=0\right)$ sería el de menor energía ( confrontar con el último párrafo de la sección 3.2.2), pero del análisis previo se ve que este no es el caso. Me gustaría recordar una observación de [92] que establece que la relación entre valores de expectación de lazos de Wilson y cuerdas en duales gravitatorios (al nivel semiclásico)

$$
\langle W\rangle \simeq e^{-A}
$$

es esquemático debido a que la adición de términos de borde a la acción de Nambu-Goto no cambia el área mínima de las soluciones pero cambia el valor de la acción clásica en algo diferente al area. En [92] esta arbitrariedad fue usada para realizar una transformación de Legendre de la acción de Nambu-Goto mostrando que la cantidad resultante, para el caso de lazos en $A d S$, está libre de divergencias lineales que provienen del comportamiento de la hoja de mundo cerca del borde de $A d S$.

También se han discutido las condiciones de concavidad (3.17) que deben ser satisfechas por cualquier potencial que pretenda describir la interacción entre quarks físicos. Duales gravitatorios genéricos tienen funciones $f(r)$ crecientes y positivos, por lo tanto las condiciones de concavidad no se satisfacen cuando la función longitud es una función creciente de la posición radial mínima, $r_{0}$, alcanzada por la cuerda. En la sección 3.2 se analizaron las funciones longitud y potencial, $L\left(r_{0}\right)$ y $V_{\text {string }}(L)$, para diferentes fondos de gravedad y se mostró que algunos de ellos conducen a soluciones del embedding de la cuerda que no satisfacen las condiciones de concavidad.

Basándome en los trabajos previos [98]-[99] he estudiado fluctuaciones lineales alrededor del embedding para testear su estabilidad. Se concluyó que cuando la solución conduce a un potencial no físico, que no satisface las condiciones (3.17), existen modos inestables ante fluctuaciones lineales. Durante el análisis se han discutido los diferentes fijados de gauge que pueden imponerse y su relación con el difeomorfismo de la acción de Nambu-Goto. Se estudiaron tres fijados de gauge naturales y se eligió trabajar en el gauge $r$ debido a que se obtienen expresiones simples y cerradas para las ecuaciones de movimiento (ver ec. (3.21)). El gauge $r$ nos conduce a un comportamiento singular para las fluctuaciones en el tip de la solución, pero refiriendo a [99] se ha mostrado que dichas fluctuaciones son físicas.

En la sección 3.3 se estudió la estabilidad de las soluciones analizadas en la sección 3.2. Se mostró mediante un análisis numérico que los fondos de gravedad $A d S_{5} \times S^{5}$, Maldacena-Núñez y Klebanov-Strassler son estables. Por otro lado para $A d S$ térmico y la generalización de Maldacena- 
Núñez de la sección 3.2.5 se encontraron modos inestables, en concordancia con el comportamiento de $L\left(r_{0}\right)$. Este último caso es patológico porque el lazo no puede estar ubicado en infinito y además, se encontró que existe un mínimo de separación más allá del cual no existen soluciones que conecten los extremos de la cuerda. En la sección 3.4 se han transformado las ecuaciones de movimiento para las fluctuaciones ( que son del tipo Sturm-Liouville), en ecuaciones de Schrödinger con el objetivo de reanalizar el problema de estabilidad. Este análisis se encontró en perfecta concordancia con los resultados obtenidos en 3.3. Se concluye que las regiones en donde se encuentran modos inestables coincide con las regiones en donde la condición de concavidad no se satisface.

En la sección 3.5 se estudio el caso de la interacción monopolo-antimonopolo en los casos de Maldacena-Nuñez y Klebanov-Strassler. Se discutió la prescripción de la conjetura para calcular lazos de 't Hooft, la cual consiste en enroscar una D3 sobre una $S^{2}$ topológica presente en la geometría. El caso de MN mostró ser inestable para todo valore de $r_{0}$. Fue factible un análisis de la fluctuación en el plano debido a que su ecuación de movimiento estaba desacoplada de las fluctuaciones angulares, se encontraron, mediante un análisis numérico, los modos inestables. El comportamiento del caso de KS, similar al $A d S$ térmico, tiene presumiblemente regiones estables e inestables, pero un análisis de fluctuaciones nos conduce a ecuaciones de movimiento acopladas que aún no se han analizado.

Se concluye que el análisis de lazos de Wilson/'t Hooft calculados en duales gravitatorios estudiando el valor de $f^{2}$ en el origen debe ser acompañado de un análisis de la función $L\left(r_{0}\right)$.

En la última sección se calcularon fluctuaciones tanto bosónicas como fermiónicas para el dual gravitatorio al valor de expectación de un lazo de Wilson- 't Hooft evaluado en la representación simétrica del grupo de gauge. En este caso aún hay trabajo por hacer, ya que puede continuarse el analisis estudiando en que representaciones del grupo de simetría transforman dichas fluctuaciones. 


\section{Capítulo 4}

\section{Superconductores Holográficos}

Luego de los trabajos $[60,62]$ la conjetura AdS/CFT se convirtió en una herramienta útil para estudiar sistemas de materia condensada. En particular, por medio del análisis de la teoría semiclásica de gravedad se han estudiado desde un punto de vista teórico sistemas de materia condensada fuertemente correlacionados (ver [71]).

En este capítulo se analizará la deformación de la geometría del espacio-tiempo dual a un superconductor $^{1}$ tipo $p$ en $3+1$ dimensiones [139] (véase [140,141] para un tratamiento similar en $4+1$ dimensiones). En el camino se reproducirá la deformación del dual gravitatorio al superconductor tipo $p+i p$ estudiado en [142]. También se usará la prescripción dada en [82, 85, 143] para calcular la entropía de entrelazamiento desde el punto de vista holográfico para ambos duales gravitatorios. Cálculos similares de la entropía de entrelazamiento en pueden encontrarse en [144, 145, 146, 147].

La superconductividad tipo $p$ es una fase de la materia que se produce cuando los electrones con momento angular relativo $j=1$ condensan formando pares de Cooper. En otras palabras, existe un vector cargado ante una simetría $U(1)$ que condensa. Este tipo de superconductores se originan a partir de electrones fuertemente correlacionados y por lo tanto la utilización de la teoría de Bardeen, Cooper y Schrieffer (BCS) no es la manera correcta de describir su dinámica microscópica. Este fenómeno es un desafío para la física teórica que, debido a la propiedad fundamental de la conjetura de describir teorías de campos fuertemente acopladas, puede ser estudiado a partir de su dual gravitatorio. Se introducirán ahora los ingredientes mínimos necesarios para reproducir la dinámica del superconductor. Este tipo de enfoque nos permite reproducir las propiedades del sistema de materia condensada sin explicar su origen microscópico.

En los trabajos $[148,149,150,151,152,153]$ fueron estudiados superconductores tipo $p$ a

\footnotetext{
${ }^{1}$ La diferencia sutíl entre un superconductor y un superfluído proviene de lo siguiente: en ambos se produce la ruptura espontanea de una simetría pero en el primer caso esta es una simetría local mientras que en el segundo caso es global. En esta tesis no se va a hacer diferencia entre uno y otro debido a que para los fenómenos considerados esta distinción no produce ninguna diferencia.
} 
partir de una teoría de cuerdas dual. Los mínimos ingredientes necesarios en la teoría gravitatoria para tener temperatura finita, potencial químico y ruptura espontánea de simetría (SSB) son: una geometría de agujero negro y un campo de gauge no Abeliano [154, 155]. Las soluciones a ser consideradas son geometrías asintóticamente AdS con un campo de gauge $S U(2)$. La SSB se realiza mediante una condición asintótica no trivial (pelo) sobre este campo de gauge. El potencial químico y la SSB provienen de prender dos direcciones independientes dentro del grupo de gauge no Abeliano. La ruptura espontánea de simetría ocurre en el lado gravitatorio a través de la formación de un condensado fuera del horizonte.

La entropía de entrelazamiento (EE) entre un subsistema $\mathcal{A}$ y su complemento $\mathcal{B}$ está definida por la entropía de von Neumann

$$
\mathcal{S}_{\mathcal{A}}=-\operatorname{Tr}_{\mathcal{A}}\left(\rho_{\mathcal{A}} \ln \rho_{\mathcal{A}}\right)
$$

Aquí $\rho_{\mathcal{A}}=\operatorname{Tr}_{\mathcal{B}}(\rho)$ es la matriz densidad obtenida de tomar la traza sobre los grados de libertad del subsistema $\mathcal{B}$ en la matrix densidad del sistema completo $\rho$. Inocentemente $S_{\mathcal{A}}$ mide la cantidad de información que está oculta dentro de $\mathcal{B}$ cuando subdividimos el sistema. Desde el punto de vista de la teoría de gravedad la EE fue conjeturada [82] proporcional al valor del area mínima para una superficie, $\gamma_{\mathcal{A}}$, en el volumen cuyo borde en infinito coincide con el borde de la región $\mathcal{A}$ (véase [143])

$$
\mathcal{S}_{\mathcal{A}}=\frac{2 \pi \operatorname{Area}\left(\gamma_{\mathcal{A}}\right)}{\kappa^{2}}
$$

Aquí $\kappa$ es la constante gravitatoria. Nótese que la entropía térmica estándar se obtiene como un caso particular de la EE cuando la región $\mathcal{A}$ es el sistema entero. En [156] los autores demostraron cómo esta técnica holográfica para calcular la EE arroja el resultado correcto al estudiarse el caso particular de una superficie esférica y a temperatura cero en teorías de campos conformes. Al final de este capítulo se calculará la EE para una geometría con forma de banda en los duales gravitatorios correspondientes a los superconductores tipo $p$ y $p+i p$.

\section{1. $\quad$ Superconductores tipo $p$ y $p+i p$}

Como ya ha sido mencionado, el dual gravitatorio a un superconductor tipo $p$ se modela con una teoría de Einstein-Yang-Mills (EYM). En [139, 157] fue estudiada dicha teoría de gravedad en $3+1$ dimensiones y en su límite de prueba, es decir, despreciando la deformación en la geometría debida

al campo de gauge. Además, estos autores mostraron que la teoría dual al superconductor tipo $p+i p$ estudiada en [142] es inestable ante pequeñas fluctuaciones siendo la configuración estable aquella para el superconductor tipo $p$. En esta sección se tendrán en cuenta las deformaciones de la geometría $3+1$ dimensional dual al superconductor $p$ producidas por el campo de gauge no 
Abeliano y se compararán los resultados con los obtenidos para el caso del $p+i p$.

Se trabajará con un grupo de gauge $S U(2)$. En el caso del $p+i p$, se propondrá un campo de gauge tal que rompa el subgrupo $U(1)$ del $S U(2)$ y la simetría rotacional $S O(3)$ del espacio pero que deje invariante un subgrupo diagonal de ellos. Por otro lado, en el dual al superconductor tipo $p$, el campo de gauge romperá ambas simetrías $U(1)$ por completo. La solución gravitatoria que describe la dinámica de acoplamiento fuerte en ambos tipos de superconductores se describe de la siguiente manera (ver figura 4.1): se forma una capa superconductora cargada fuera del horizonte producida por la competencia entre la repulsión eléctrica (con el agujero negro cargado) y el potencial gravitatorio de la geometría asintóticamente AdS. A temperaturas suficientemente altas no se produce pelo fuera del agujero negro y la solución es AdS-Reissner-Nordström (AdSRN). Por debajo de una temperatura crítica $T_{c}$ se genera un campo de gauge no trivial con potencial químico no nulo sobre el borde de la geometría y aparece un condesado sin necesidad de tener una fuente para el mismo lo cual origina un rompimiento espontáneo de la simetría de gauge $S U(2)$.

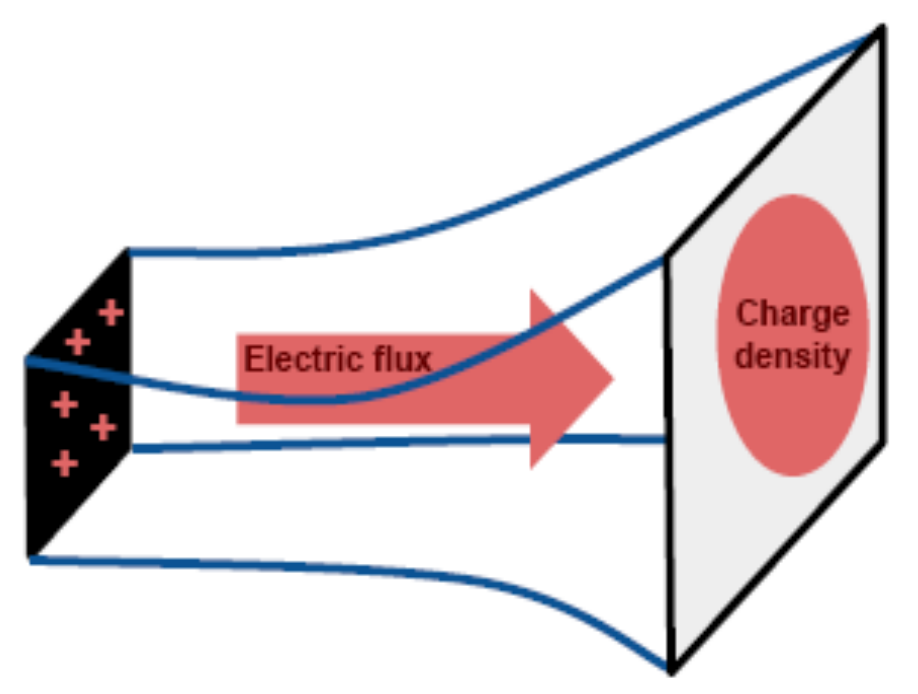

Figura 4.1: El agujero negro AdSRN plano. La fuente de la densidad de carga se encuentra en el horizonte del agujero negro.

\subsubsection{Superconductor tipo $p$ en $2+1$ dimensiones}

\section{Solución}

Comencemos a partir de una teoría de Yang-Mills en $3+1$ dimensiones y con grupo de gauge $S U(2)$ en un espacio AdS (ver [158]), su densidad Lagrangiana es 


$$
\kappa_{(4)}^{2} \mathcal{L}=R-2 \Lambda-\frac{1}{4} \operatorname{Tr}\left(F_{\mu \nu} F^{\mu \nu}\right)
$$

en donde $\Lambda=-\frac{3}{\hat{R}^{2}}, \kappa_{(4)}$ es la constante gravitacional en cuatro dimensiones y el $F_{\mu \nu}$ para el campo de gauge es

$$
F_{\mu \nu}^{a}=\partial_{\mu} A_{\nu}^{a}-\partial_{\nu} A_{\mu}^{a}+g_{Y M} \epsilon^{a b c} A_{\mu}^{b} A_{\nu}^{c}
$$

con $g_{Y M}=\frac{\hat{g}_{Y M}}{\kappa_{(4)}}$ denotando al parámetro que mide la deformación de la geometría y $\hat{g}_{Y M}$ el acoplamiento de Yang-Mills usual. Se usarán letras latinas como índice sobre el grupo $S U(2)$ y letras griegas para denotar coordenadas del espacio-tiempo. Escaleando el campo de gauge como $\tilde{A}=\frac{A}{g_{Y M}}$ se puede ver que el límite de $g_{Y M}$ grande corresponde al límite de prueba del campo de gauge. Aproximadamente uno puede pensar que $\frac{1}{\widehat{g}_{Y M}^{2}}$ cuenta el número de grados de libertad cargados ante el $S U(2)$ de la teoría dual. Además, $\frac{1}{\kappa_{(4)}^{2}}$ cuenta el número total de grados de libertad. Considerar la deformación producida por el campo de gauge significa que el número de grados de libertad de los estados cargados es del mismo orden que el número de grados de libertad del sistema.

Las ecuaciones de movimiento obtenidas a partir de minimizar la acción son

$$
\begin{aligned}
G_{\mu \nu}=R_{\mu \nu}-\frac{1}{2} g_{\mu \nu} R & =\frac{3}{R^{2}} g_{\mu \nu}+\frac{1}{2} \operatorname{Tr}\left[F_{\mu \gamma} F_{\nu}^{\gamma}\right]-\frac{g_{\mu \nu}}{8} \operatorname{Tr}\left[F_{\gamma \rho} F^{\gamma \rho}\right] \\
D_{\mu} F^{\mu \nu} & =0
\end{aligned}
$$

y con el objetivo de resolverlas se propondrá la siguiente solución [140, 159]

$$
d s^{2}=-M(r) \sigma(r)^{2} d t^{2}+\frac{1}{M(r)} d r^{2}+r^{2} h(r)^{2} d x^{2}+r^{2} h(r)^{-2} d y^{2},
$$

para la geometría de fondo, y

$$
A=\phi(r) \tau^{3} d t+\omega(r) \tau^{1} d x
$$

para el campo de gauge. Aquí se ha usado la notación matricial $A=A_{\mu}^{a} \tau^{a} d x^{\mu} \operatorname{con} \tau^{a}=\frac{\sigma^{a}}{2 i}$ y $\sigma^{a}$ las matrices de Pauli usuales, los generadores de $S U(2)$ satisfacen $\left[\tau^{a}, \tau^{b}\right]=\epsilon^{a b c} \tau^{c}$. Un solución que satisfaga que $\omega \neq 0$ en el campo de gauge (4.8) romperá la simetría $U(1)$ asociada con rotaciones alrededor de $\tau^{3}$ (usualmente denominada $U(1)_{3}$ ). Además si $h \neq 0$ se romperá la simetría $U(1)_{x y}$ asociada a rotaciones en el plano $x y$. A temperaturas suficientemente altas se espera que no haya 
pelo fuera del agujero negro y la solución con condensado nulo es AdSRN con

$$
\begin{aligned}
\omega(r) & =0, \\
h(r) & =1 \\
\sigma(r) & =1, \\
\phi(r) & =\mu\left(1-\frac{r_{h}}{r}\right), \\
M(r) & =r^{2}+\frac{\mu^{2} r_{h}^{2}}{r^{2}}-\left(\frac{\mu^{2}}{8}+r_{h}^{2}\right) \frac{r_{h}}{r} .
\end{aligned}
$$

Reemplazando la solución propuesta en las ecuaciones de movimiento para la acción de EYM se obtienen 5 ecuaciones, tres de ellas son ecuaciones diferenciales de segundo orden y las dos restantes son ligaduras de primer orden

$$
\begin{aligned}
M^{\prime} & =\frac{3 r}{\hat{R}^{2}}-\frac{1}{8 \sigma^{2}}\left(\frac{g_{Y M}^{2} \phi^{2} \omega^{2}}{r h^{2} M}+r \phi^{\prime 2}\right)-M\left(\frac{1}{r}+\frac{r h^{\prime 2}}{h^{2}}+\frac{\omega^{\prime 2}}{8 r h^{2}}\right) \\
\sigma^{\prime} & =\frac{\sigma}{h^{2}}\left(r h^{\prime 2}+\frac{\omega^{\prime 2}}{8 r}\right)+\frac{g_{Y M}^{2} \phi^{2} \omega^{2}}{8 r M^{2} h^{2} \sigma} ; \\
h^{\prime \prime} & =\frac{1}{8 r^{2} h}\left(-\omega^{\prime 2}+\frac{g_{Y M}^{2} \phi^{2} \omega^{2}}{M^{2} \sigma^{2}}\right)-h^{\prime}\left(\frac{2}{r}-\frac{h^{\prime}}{h}+\frac{M^{\prime}}{M}+\frac{\sigma^{\prime}}{\sigma}\right) ; \\
\omega^{\prime \prime} & =-\frac{g_{Y M}^{2} \phi^{2} \omega}{M^{2} \sigma^{2}}+\omega^{\prime}\left(\frac{2 h^{\prime}}{h}-\frac{M^{\prime}}{M}-\frac{\sigma^{\prime}}{\sigma}\right) ; \\
\phi^{\prime \prime} & =\frac{g_{Y M}^{2} \phi \omega^{2}}{r^{2} h^{2} M}-\phi^{\prime}\left(\frac{2}{r}-\frac{\sigma^{\prime}}{\sigma}\right) .
\end{aligned}
$$

Este sistema de ecuaciones goza de 4 simetrías de escala que serán útiles cuando intentemos resolverlas numéricamente, ellas son

1. $\sigma \rightarrow \lambda \sigma, \quad \phi \rightarrow \lambda \phi$

2. $\omega \rightarrow \lambda \omega, \quad h \rightarrow \lambda h$

3. $M \rightarrow \lambda^{-2} M, \quad \sigma \rightarrow \lambda \sigma, \quad g_{Y M} \rightarrow \lambda^{-1} g_{Y M}, \quad \hat{R} \rightarrow \lambda \hat{R}$

4. $M \rightarrow \lambda^{2} M, \quad r \rightarrow \lambda r, \quad \phi \rightarrow \lambda \phi, \quad \omega \rightarrow \lambda \omega$

Usando estas simetrías de escala podemos elegir $\hat{R}=r_{h}=1$ y fijar el valor en el borde de las funciones de la métrica $\sigma(\infty)=h(\infty)=1$. La geometría y el campo de gauge deben ser regulares en el horizonte, esto motiva la siguiente expansión en el IR ( $r$ pequeño)

$$
\begin{aligned}
M & =M_{1}\left(r-r_{h}\right)+M_{2}\left(r-r_{h}\right)^{2}+\ldots \\
h & =h_{0}+h_{2}\left(r-r_{h}\right)^{2}+\ldots \\
\sigma & =\sigma_{0}+\sigma_{1}\left(r-r_{h}\right)+\sigma_{2}\left(r-r_{h}\right)^{2}+\ldots \\
\omega & =\omega_{0}+\omega_{2}\left(r-r_{h}\right)^{2}+\omega_{3}\left(r-r_{h}\right)^{3}+\ldots \\
\phi & =\phi_{1}\left(r-r_{h}\right)+\phi_{2}\left(r-r_{h}\right)^{2}+\ldots
\end{aligned}
$$


Por otro lado, en el UV ( $r$ grande) el desarrollo deseado es

$$
\begin{aligned}
M & =r^{2}+\frac{M_{1}^{b}}{r}+\frac{\left(\omega_{1}^{b}\right)^{2}+\rho^{2}}{8 r^{2}}+\ldots \\
h & =1+\frac{h_{3}^{b}}{r^{3}}-\frac{\left(\omega_{1}^{b}\right)^{2}}{32 r^{4}}+\ldots \\
\sigma & =1-\frac{\left(\omega_{1}^{b}\right)^{2}}{32 r^{4}}+\ldots \\
\omega & =\omega_{0}^{b}+\frac{\omega_{1}^{b}}{r}-\frac{g_{Y M}^{2} \mu^{2} \omega_{1}^{b}}{6 r^{3}}+\ldots \\
\phi & =\mu+\frac{\rho}{r}+\frac{g_{Y M}^{2} \mu^{2} \omega_{1}^{b}}{12 r^{4}}+\ldots
\end{aligned}
$$

Para dar cuenta de la SSB, se buscan soluciones en donde la componente no normalizable se anule $\omega_{0}^{b}=0$. El diccionario estándar de AdS/CFT nos permite interpretar al valor de borde y al subleading (segundo termino más relevante en la expansión) de $\phi$ como el potencial químico $\mu$ y la densidad de carga $\rho$ de la teoría de campos dual [160]. Además, el coeficiente sub-leading $M_{1}^{b}$ en la expansión en el borde de $g_{t t}$ coincide con la acción Euclídea regularizada evaluada en la solución de las ecuaciones de movimiento [60]. El coeficiente normalizable en $\omega$ es dual al valor de expectación de vació de la corriente $\left\langle J_{x}^{1}\right\rangle \propto \omega_{1}^{b}$ y hace las veces de parámetro de orden para el sistema.

Las soluciones de las ecuaciones (4.10) dependen de cuatro coeficientes IR $\phi_{1}, \omega_{0}, h_{0}, \sigma_{0}$ y del parámetro de deformación $g_{Y M}$. Todos los otros coeficientes en (4.11) pueden ser escritos en términos de estos. Se procederá a integrar las ecuaciones de movimiento numéricamente desde el horizonte hacia el borde usando un método de shooting con el objetivo de tener el comportamiento asintótico deseado. Se exploró el rango $g_{Y M} \in[0,85,24]$ y se observó que el comportamiento de las funciones no cambia cualitativamente al variar $g_{Y M}$. En las figuras 4.2 y 4.3 se muestran las soluciones de (4.10) con condiciones de borde (4.12). Se ha usado el potencial químico $\mu$ para adimensionalizar. Esto significa que se esta trabajando en el conjunto gran canónico.

\section{Termodinámica}

En esta sección se calcularán las cantidades termodinámicas asociadas con las soluciones. A partir del estudio de la función potencial en el conjunto ${ }^{2}$ gran canónico se observará una transición de fase de segundo orden entre una fase superconductora y una normal.

La temperatura de la teoría de campos dual está dada por la temperatura de Hawking del agujero negro

$$
T=\frac{M_{1} \sigma_{0}}{2 \pi}=\frac{1}{16 \pi}\left(24 \sigma_{0}^{2}-\phi_{1}^{2}\right) r_{h}
$$

\footnotetext{
${ }^{2}$ Para pasar del conjunto canónico ( $\mu$ fijo) con energía libre $\Omega$, al conjunto gran canónico ( $\rho$ fijo) con energía libre $F$, se debe agregar un termino de borde a la acción Euclídea. Esto cambia el problema variacional e implica la relación de Gibbs $F=\Omega+\mu \rho$.
} 

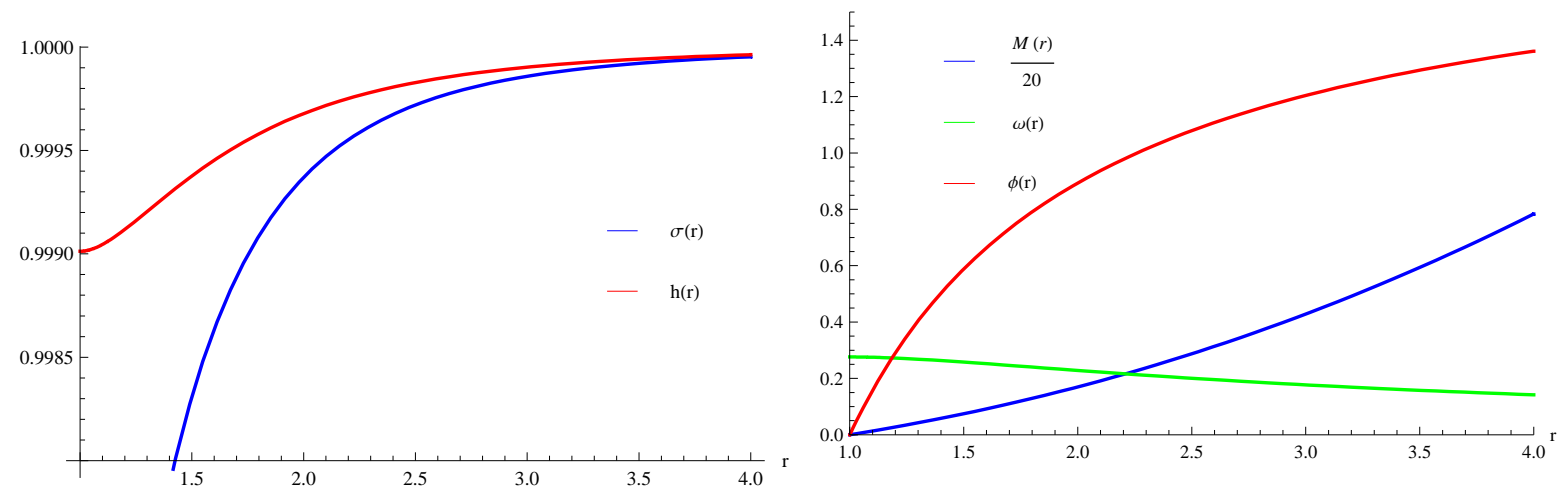

Figura 4.2: Las funciones adimensionales de la métrica $\sigma(r)$ y $h(r)$ para $g_{Y M}=2$ y $T=0,2312 \mu$.

Figura 4.3: Las funciones adimensionales de la métrica $M(r)$ y las funciones del campo de gauge $\omega(r)$ y $\phi(r)$ para $g_{Y M}=2, T=0,2312 \mu$.

en donde la segunda igualdad proviene de la consistencia de la expansión en serie(4.11) que relaciona el coeficiente $M_{1}$ con $\sigma_{0}$ y $\phi_{1}$. El área del horizonte, $A_{h}$, conduce a la entropía

$$
S=\frac{2 \pi}{\kappa_{(4)}^{2}} A_{h}=\frac{2 \pi^{2} V T^{2}}{\kappa_{(4)}^{2}} \frac{12^{2}}{\left(24 \sigma_{0}^{2}-\phi_{1}^{2}\right)^{2}}
$$

en donde $V=\int d x d y$. En la figura 4.4 se muestra el parámetro de orden $\omega_{1}^{b}$ (es decir, el valor de expectación de la corriente $\left.\left\langle J_{x}^{1}\right\rangle\right)$ como función de la temperatura. Nótese que a $T=T_{c}$ el condensado se anula mostrando la desaparición de el estado superconductor para $T>T_{c}$. A partir de los resultados numéricos se encuentra que cerca de la temperatura crítica $T_{c}$ la corriente satisface $\left\langle J_{1}^{x}\right\rangle \propto\left(1-\frac{T}{T_{c}}\right)^{1 / 2}$ y por lo tanto el valor del exponente crítico es $1 / 2$. La figura 4.5 muestra el comportamiento de la entropía de Bekenstein-Hawking (4.14) como función de la temperatura para mi solución y la del agujero negro AdSRN.

La correspondencia AdS/CFT identifica la acción Euclídea on-shell de la teoría de gravedad $S_{E}$ multiplicada por la temperatura $T$ como la función potencial $\Omega$ del sistema en el conjunto gran canónico. Para calcularla, se hará una continuación a signatura Euclídea y se compactificará el tiempo con periodo $\frac{1}{T}$ para evitar singularidades. La acción on-shell tiene un factor $\frac{1}{T}$ proveniente de la integración en la coordenada temporal, escribiendo $S_{o n-s h e l l}=\frac{\tilde{S}_{b u l k}}{T}$ se tiene

$$
\tilde{S}_{b u l k}=-\int d x d y d r \sqrt{-g} \mathcal{L}
$$

en donde la densidad Lagrangiana está escrita en (4.3). La componente yy del tensor energíamomento es proporcional a la métrica y por lo tanto las ecuaciones de Einstein (4.5) implican que

$$
G_{y y}=\frac{r^{2}}{2 h^{2}}\left(\kappa_{(4)}^{2} \mathcal{L}-R\right)
$$




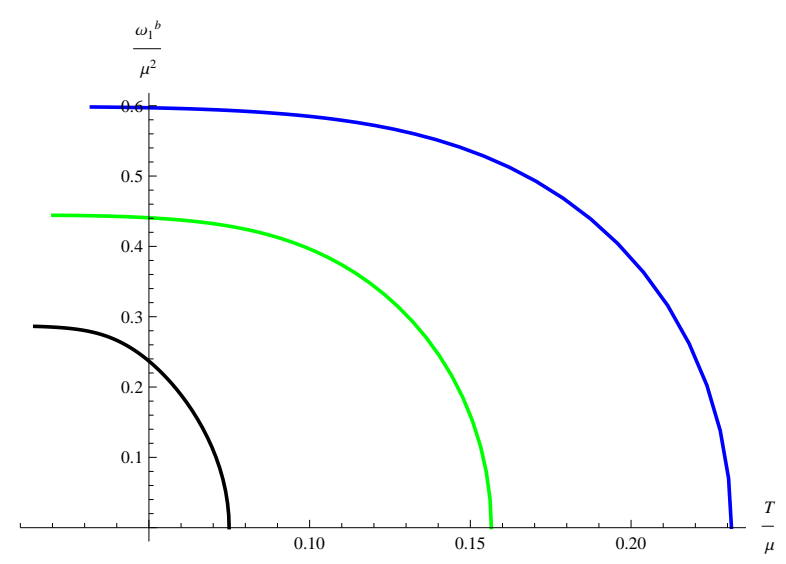

Figura 4.4: La figura muestra el comportamiento del coeficiente normalizable de la función $\omega$, el cual es proporcional al condensado $\left\langle J_{x}^{1}\right\rangle$. Las líneas negra, verde y azul refieren a soluciones con $g_{Y M}=1,1,5,2$ y $T_{c}=0,0749,0,1565,0,2312$ respectivamente. Nótese que el condensado se anula para $T>T_{c}$.

Luego, tenemos

$$
G_{\mu}^{\mu}=-R=G_{r}^{r}+G_{t}^{t}+G_{x}^{x}+\frac{1}{2}\left(\kappa_{(4)}^{2} \mathcal{L}-R\right)
$$

a partir de lo que se obtiene

$$
\mathcal{L}=\frac{2}{r^{2} \sigma \kappa_{(4)}^{2}}\left[\frac{r^{3} M \sigma}{h}\left(\frac{h}{r}\right)^{\prime}\right]^{\prime}
$$

en donde ' denota derivada con respecto a la coordenada holográfica $r$. Luego, la contribución a la acción on-shell (4.15) proveniente del volumen del espacio-tiempo se puede escribir como

$$
\tilde{S}_{b u l k}=-\int d x d y d r \sqrt{-g} \mathcal{L}=-\frac{2 V}{\kappa_{(4)}^{2}}\left[\frac{r^{3} M \sigma}{h}\left(\frac{h}{r}\right)^{\prime}\right]_{r=r_{\infty}}
$$

en donde $r_{\infty}$ es el borde del espacio. Como es usual, para tener un problema variacional bien definido cuando se imponen condiciones de borde Dirichlet en la métrica necesitamos agregar a la acción el término de Gibbons-Hawking

$$
\tilde{S}_{G H}=-\frac{1}{\kappa_{(4)}^{2}} \int d x d y \sqrt{-g_{\infty}} \nabla_{\mu} n^{\mu}=-\frac{V}{\kappa_{(4)}^{2}} r^{2} \sigma\left[\frac{M^{\prime}}{2}+M\left(\frac{\sigma^{\prime}}{\sigma}+\frac{2}{r}\right)\right]_{r=r_{\infty}},
$$

siendo $n^{\mu} d x_{\mu}=\sqrt{M} d r$ el vector unitario normal al borde y $g_{\infty}$ el determinante de la métrica inducida sobre el borde. Precisamente en $r=r_{\infty}$ (4.20) diverge y por lo tanto debe ser regularizada mediante la suma de contra-términos de borde

$$
\tilde{S}_{c t}=\frac{1}{\kappa_{(4)}^{2}} \int d x d y \sqrt{-g_{\infty}}=\frac{V}{\kappa_{(4)}^{2}}\left[r^{2} \sqrt{M} \sigma\right]_{r=r_{\infty}}
$$



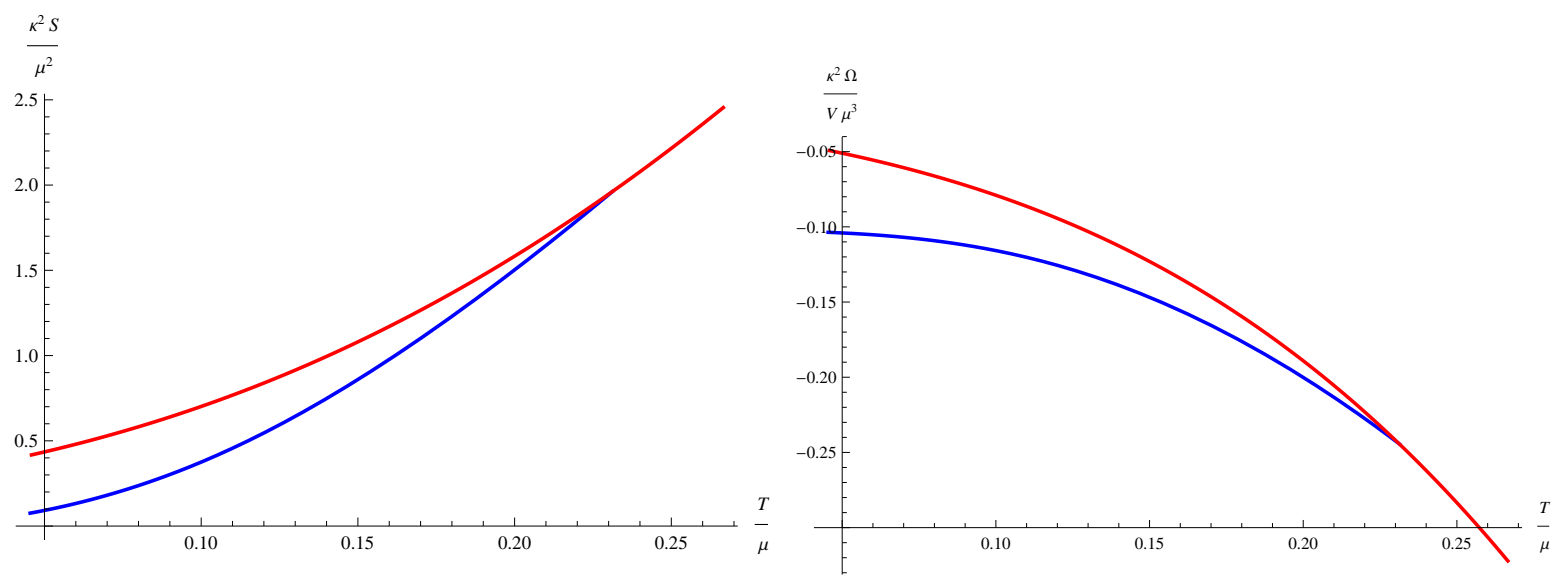

Figura 4.5: Entropía como función de la temperatura. La línea azul es para la fase superconductora con $g_{Y M}=2$ y la línea roja refiere a la fase normal (geometría AdSRN). Hay una transición de Figura 4.6: Función potencial $\Omega$ calculada a partir de (4.22) como unción de la temperatura $T$ para $g_{Y M}=2$. La línea roja es el potencial para la solución de RN y la azul para la fase superconductora. fase de segundo orden para $T=T_{c}=0,2312$.

Finalmente, el potencial termodinámico dual $\Omega$ resulta

$$
\begin{aligned}
\Omega & =\lim _{r_{\infty} \rightarrow \infty} \tilde{S}_{\text {on-shell }} \\
& =\lim _{r_{\infty} \rightarrow \infty}\left(\tilde{S}_{b u l k}+\tilde{S}_{G H}+\tilde{S}_{c t}\right)
\end{aligned}
$$

Luego de regularizar la acción el potencial $\Omega$ coincide con el valor sub-leading de la componente $g_{t t}$ de la métrica de fondo, es decir, $\Omega=M_{1}^{b}$ [60]. Se ha verificado la solución numérica calculando $\Omega$ de ambas maneras y se encontró una concordancia excelente. En la figura 4.6 se muestra el potencial (4.22) como función de la temperatura. Como se mencionó anteriormente se produce una transición de fase de segundo orden en $T=T_{c}$ : el gran potencial y la entropía son continuas pero $S$ no es diferenciable. Por debajo de $T_{c}$ el sistema se encuentra en la fase superconductora, al incrementar la temperatura por sobre $T_{c}$ la geometría de AdSRN domina la energía libre, lo que modela la transición de la fase superconductora a la normal.

\subsection{2. $\quad$ Superconductores tipo $p+i p$}

En esta sección se recopilarán los resultados de [142] y se compararán con los resultados obtenidos en la sección anterior. Se encontró que a $T=T_{c}$ el sistema tiene una transición de fase de segundo orden y que en todo el rango de temperatura el gran potencial del superconductor tipo $p$ es menor que el del $p+i p$. Esto implica que la fase estable del sistema es la tipo $p$, lo cual concuerda con el análisis de estabilidad desarrollado en [139]. 


\section{Solución}

La geometría y el campo de gauge para modelar la solución son

$$
\begin{aligned}
d s^{2} & =-M(r) d t^{2}+r^{2} h(r)^{2}\left(d x^{2}+d y^{2}\right)+\frac{d r^{2}}{M(r)} \\
A & =\phi(r) \tau^{3} d t+\omega(r)\left(\tau^{1} d x+\tau^{2} d y\right) .
\end{aligned}
$$

Aquí hay un diferencia importante con el superconductor tipo $p$ de la sección previa. Ahora el campo de gauge rompe el grupo $U(1)_{3} \times U(1)_{x y}$ dejando invariante una combinación diagonal. En la sección anterior el campo de gauge rompía por completo el grupo $U(1)_{3} \times U(1)_{x y}$. Esto nos permite proponer una métrica totalmente simétrica en el plano $x y$.

Las 5 ecuaciones de movimiento obtenidas se dividen en 4 ecuaciones diferenciales de segundo orden y una ligadura de primer orden proveniente de la componente $r r$ de la ecuaciones de Einstein

$$
\begin{aligned}
h^{\prime \prime} & =-\frac{h}{2}\left[\frac{1}{r^{2}}-\frac{3}{\hat{R}^{2} M}+\frac{M^{\prime}}{r M}+\frac{\phi^{\prime 2}}{8 M}+\frac{\omega^{\prime 2}}{4 r^{2} h^{2}}\right]-\frac{h^{\prime}}{2}\left[\frac{6}{r}+\frac{h^{\prime}}{h}+\frac{M^{\prime}}{M}\right]-\frac{g_{Y M}^{2} \omega^{2}}{8 r^{2} h M}\left[\frac{\phi^{2}}{M}+\frac{\omega^{2}}{2 r^{2} h^{2}}\right] \\
M^{\prime \prime} & =\frac{3}{\hat{R}^{2}}+\frac{M}{r}\left[-\frac{M^{\prime}}{M}+\frac{1}{r}+\frac{\omega^{\prime 2}}{4 r h^{2}}\right]-\frac{h^{\prime}}{h}\left[M^{\prime}-\frac{h^{\prime}}{h}-\frac{2}{r}\right]+\frac{3}{8} \phi^{\prime 2}+\frac{g_{Y M}^{2} \omega^{2}}{4 r^{2} h^{2}}\left[\frac{\phi^{2}}{M}+\frac{3 \omega^{2}}{2 r^{2} h^{2}}\right] \\
\omega^{\prime \prime} & =\frac{g_{Y M}^{2} \omega}{M}\left[\frac{\omega^{2}}{r^{2} h^{2}}-\frac{\phi^{2}}{M}\right]-\frac{M^{\prime} \omega^{\prime}}{M} \\
\phi^{\prime \prime} & =\frac{2 g_{Y M}^{2} \phi \omega^{2}}{r^{2} h^{2} M}-2 \phi^{\prime}\left[\frac{1}{r}+\frac{h^{\prime}}{h}\right] \\
0 & =-\frac{3}{\hat{R}^{2}}+\frac{M}{r^{2}}\left[1-\frac{\omega^{\prime 2}}{4 h^{2}}+\frac{M^{\prime}}{M} r\right]+\frac{h^{\prime}}{h}\left[M\left(\frac{2}{r}+\frac{h^{\prime}}{h}\right)+M^{\prime}\right]+\frac{1}{8} \phi^{\prime 2} .
\end{aligned}
$$

Las ecuaciones tienen tres simetrías de escala que nos ayudarán a resolver el sistema numéricamente. Estas son

1. $\omega \rightarrow \lambda \omega, \quad h \rightarrow \lambda h$

2. $M \rightarrow \lambda^{-2} M, \quad \phi \rightarrow \frac{\phi}{\lambda}, \quad \hat{R} \rightarrow \lambda \hat{R}, \quad g_{Y M} \rightarrow \frac{g_{Y M}}{\lambda}$

3. $M \rightarrow \lambda^{2} M, \quad h \rightarrow \frac{h}{\lambda}, \quad \phi \rightarrow \lambda \phi, \quad r \rightarrow \lambda r$

y permiten escoger $R=r_{h}=1$ y fijar el valor de $h(r)$ en el borde a $h(\infty)=1$. El comportamiento en el IR de estas ecuaciones es el mismo que para un agujero negro cargado

$$
\begin{aligned}
M & =M_{1}\left(r-r_{h}\right)+M_{2}\left(r-r_{h}\right)^{2}+\ldots \\
h & =h_{0}+h_{1}\left(r-r_{h}\right)+h_{2}\left(r-r_{h}\right)^{2}+\ldots \\
\omega & =\omega_{0}+\omega_{1}\left(r-r_{h}\right)+\omega_{2}\left(r-r_{h}\right)^{2}+\ldots \\
\phi & =\phi_{1}\left(r-r_{h}\right)+\phi_{2}\left(r-r_{h}\right)^{2}+\ldots
\end{aligned}
$$


en donde, como antes, se impuso que el potencial de Maxwell $\phi$ se anule en el horizonte con el objeto de obtener un campo de gauge bien definido cuando se realize la continuación Euclídea. En el UV se pedirá

$$
\begin{aligned}
M & =r^{2}+2 h_{1}^{b} r+\left(h_{1}^{b}\right)^{2}+\frac{M_{1}^{b}}{r}+\frac{-8 h_{1}^{b} M_{1}^{b}+\rho^{2}+2\left(\omega_{1}^{b}\right) / 3}{8 r^{2}}+\ldots \\
h & =1+\frac{h_{1}^{b}}{r}-\frac{\left(\omega_{1}^{b}\right)^{2}}{48 r^{4}}+\ldots \\
\omega & =\frac{\omega_{1}^{b}}{r}-\frac{h_{1}^{b} \omega_{1}^{b}}{r^{2}}+\ldots \\
\phi & =\mu+\frac{\rho}{r}-\frac{\rho h_{1}^{b}}{r^{2}}+\ldots
\end{aligned}
$$

Nótese que para que ocurra una ruptura espontánea de simetría no se permite un término no normalizable en $\omega$. Como antes, las simetrías de escala (3) permiten fijar $h_{0}^{b}=1$. En la figura 4.7 se muestra el comportamiento de las solución y en la figura 4.8 se grafica el parámetro de orden $\left\langle J_{x}^{1}\right\rangle>\propto \omega_{1}^{b}$ como una función de la temperatura. Para $T=T_{c}$ ambos condensados se anulan y ocurre una transición de fase de segundo orden. Nótese que los valores del condensado para el caso del $p+i p$ son menores que aquellos para el superconductor tipo $p$.

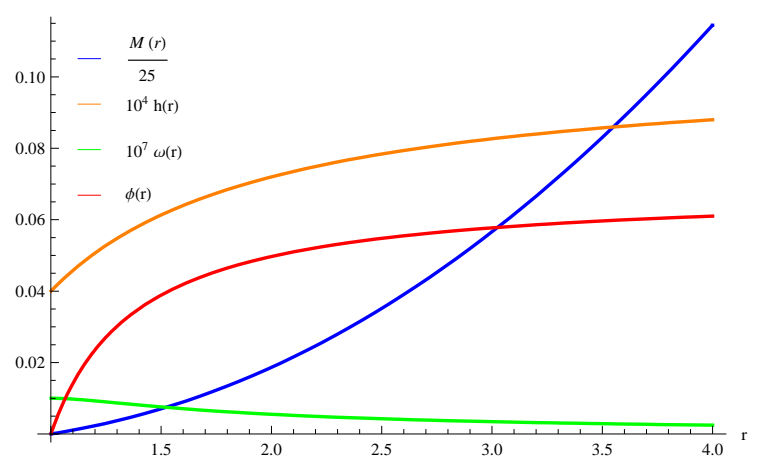

Figura 4.7: Comportamiento de las funciones adimensionales $M(r), h(r), \omega(r)$ y $\phi(r)$, para $g_{Y M}=$ $2, T=0,2312 \mu$.

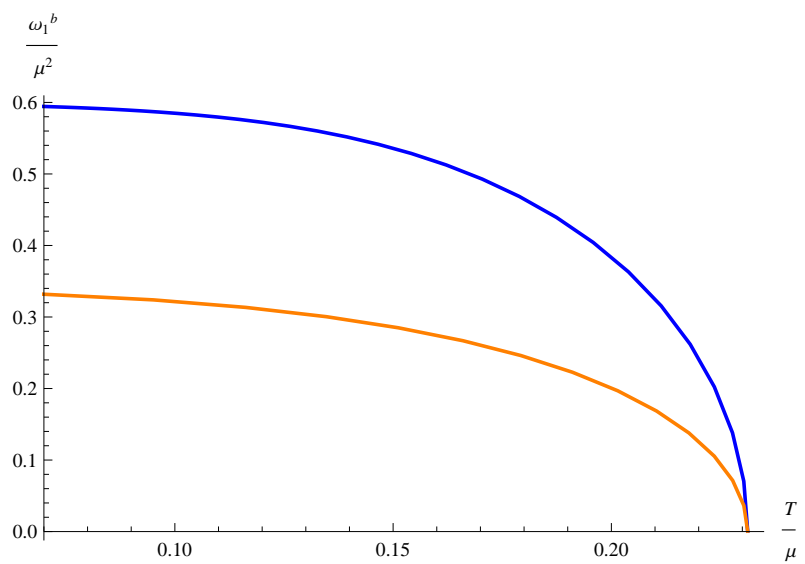

Figura 4.8: El valor de expectación $\left\langle J_{x}^{1}\right\rangle \propto \omega_{1}^{b}$ de la teoría de campos dual como función de la temperatura para el superconductor tipo $p$ (línea azul) y tipo $p+i p$ (línea naranja) para $g_{Y M}=2$. Se anula para $T>T_{c}=0,2312$, lo cual sugiere la existencia de una transición de fase entre el estado superconductor y el normal. 


\section{Termodinámica}

La temperatura asociada a la solución de fondo es proporcional a la derivada de la componente $g_{t t}$ de la métrica evaluada en el horizonte. En este caso se tiene

$$
T=\frac{M_{1}}{2 \pi}
$$

La formula de Bekenstein-Hawking, que relaciona la entropía con el area del horizonte del agujero negro, en este caso es

$$
S=\frac{2 \pi}{\kappa_{(4)}^{2}} A_{h}=\frac{2 \pi}{\kappa_{(4)}^{2}} r_{h}^{2} h_{0}^{2} .
$$

En la figura 4.9 se muestra la entropía en el caso del superconductor tipo $p+i p$ (linea naranja), del tipo $p$ (línea azul) y del agujero negro de RN (línea roja).

El grand potencial $\Omega$ está dado por el coeficiente del término sub-leading de la función $g_{t t}$ de la métrica,

$$
\Omega=\frac{V M_{1}^{b}}{\kappa_{(4)}^{2}}
$$

Este potencial se dibuja en la figura 4.10, en donde claramente se muestra que para cualquier valor de la temperatura la solución tipo $p$ (azul) es preferida por sobre la $p+i p$ (naranja) debido a su menor energía libre. Para $T>T_{c}$ el sistema se encuentra en su fase normal (en rojo) y el condensado se anula (ver figura 4.8).

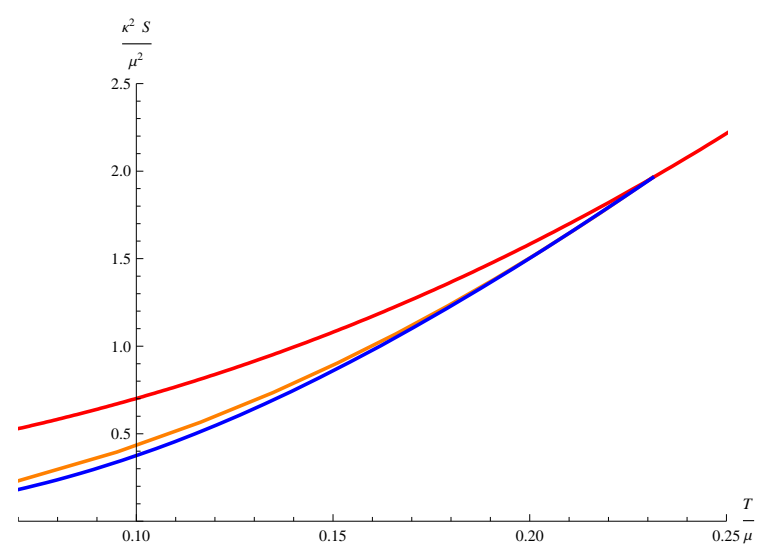

Figura 4.9: Entropía de Bekenstein-Hawking para el agujero negro de RN (rojo) y las soluciones de superconductro tipo $p$ (azul) y $p+i p$ (naranja) con $g_{Y M}=2$ y $T_{c}=0,2312$. Hay una transición de fase de segundo orden para ambos superconductores en $T=T_{c}$.

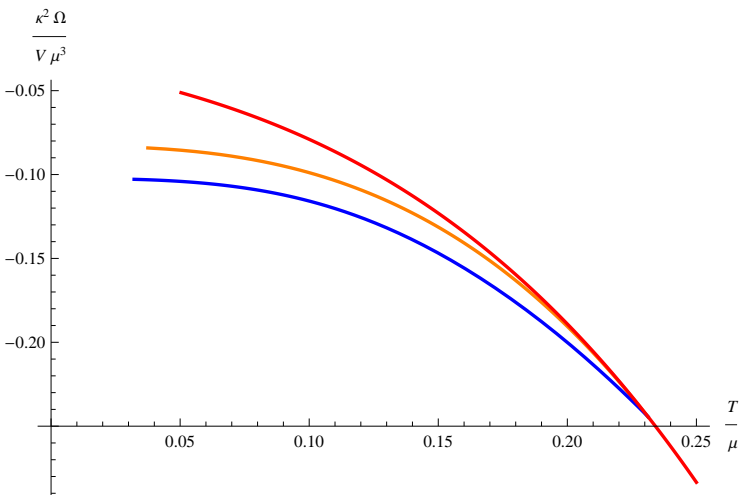

Figura 4.10: Potencial gran canónico como función de la temperatura. Solución de RN (rojo), $p+i p$ (naranja) y $p$ (azul). Para todo rango de temperaturas por debajo de $T_{c}$ la solución de superconductor tipo $p$ es preferida por sobre la $p+i p\left(g_{Y M}=2\right.$ y $\left.T_{c}=0,2312\right)$. 


\subsection{Entropía de entrelazamiento}

Una prescripción holográfica para calcular la entropía de entrelazamiento (EE) en el espacio $\mathrm{AdS}_{d+1}$ dual a una $\mathrm{CFT}_{d}$ fue propuesta en [82] en términos de superficies mínimas. La prescripción involucra subdividir el sistema en dos regiones, $\mathcal{A}$ y su complemento $\mathcal{B}$, y encontrar las superficie estática mínima $d-1$ dimensional (a tiempo constante) $\gamma_{\mathcal{A}}$ tal que su borde coincide con el borde del subsistema $\mathcal{A}$ (ver figura 4.11).

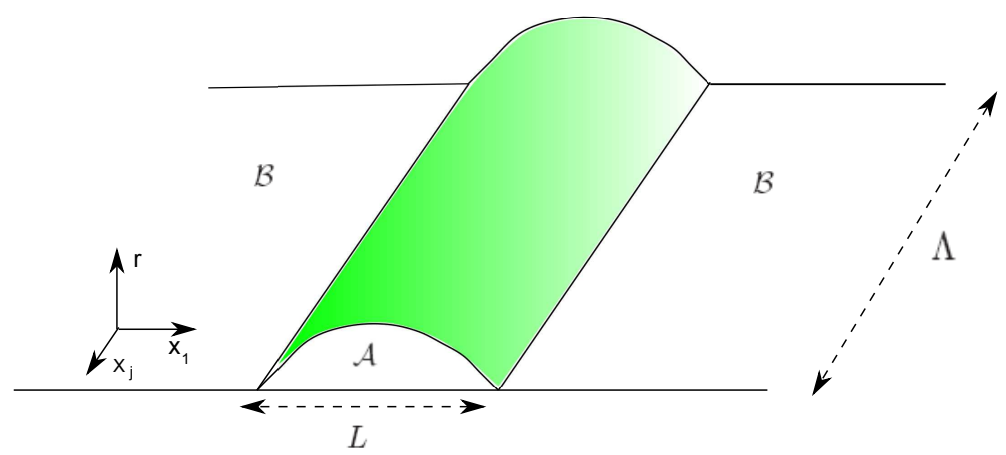

Figura 4.11: Diagrama de la región en forma de banda $\mathcal{A}$ usada para calcular la entropía de entrelazamiento.

La entropía de entrelazamiento entre las dos regiones es el area clásica de $\gamma_{\mathcal{A}}$,

$$
\mathcal{S}_{\mathcal{A}}=\frac{2 \pi}{\kappa_{(d+1)}^{2}} \int_{\gamma_{\mathcal{A}}} d^{(d-1)} \sigma \sqrt{g_{\text {ind }}^{(d-1)}}
$$

en donde $g_{i n d}^{(d-1)}$ es la métrica inducida sobre la superficie y $\kappa_{(d+1)}^{2}$ es la constante gravitatoria en $d+1$ dimensiones.

En [161] y [162] la EE fue calculada para fondos gravitatorios duales a teorías de gauge confinantes y a geometrías de agujero negro respectivamente. En esta sección se realizará un cálculo similar para un fondo de gravedad general y se aplicará la prescripción a las soluciones de superconductores tipo $p$ y $p+i p$ encontrados en la sección 4.1. Nótese que la prescripción es muy similar a la realizada en $[45,46]$ para calcular valores de expectación de lazos de Wilson (ver capítulo 3 ).

Escribamos la métrica de fondo $d+1$ dimensional como

$$
d s_{d+1}^{2}=-g_{t t}(r) d t^{2}+g_{x_{i} x_{i}}(r) d x_{i}^{2}+g_{r r}(r) d r^{2}, \quad i=1 \ldots d-1,
$$

en donde $r$ es la coordenada holográfica. La región de interés será una banda recta en la dirección $x_{j}$ con ancho $L$ en la dirección $x_{1}$. La forma de embeber la superficie estáticamente es $x_{1}=x_{1}(\zeta), x_{j}=$ $\zeta_{j}, r=r(\zeta)$, con $j=2, \ldots, d-1$. Aún se tiene invarianza ante difeomorfismos en $\mathcal{S}_{\mathcal{A}}$, dependiendo 
del contexto esta puede ser fijada como $x_{1}=\zeta$ (embedding global) o $r=\zeta$. La entropía (4.30) es

$$
\mathcal{S}_{\mathcal{A}}=\frac{2 \pi \Lambda}{\kappa_{(d+1)}^{2}} \int d \zeta \sqrt{g_{x_{2} x_{2}}(r) \ldots g_{x_{d-1} x_{d-1}}(r)} \sqrt{g_{r r}(r) r^{\prime 2}+g_{x_{1} x_{1}}(r) x_{1}^{\prime 2}}
$$

en donde $\Lambda=\int d \zeta_{2} \ldots d \zeta_{d-1}$ y el símbolo ' denota derivadas en $\chi$.

Definiendo $g_{\chi \chi}(r)=g_{x_{2} x_{2}}(r) \ldots g_{x_{d-1} x_{d-1}}(r)$ y las funciones

$$
f^{2}(r)=g_{\chi \chi}(r) g_{x_{1} x_{1}}(r), \quad \eta^{2}(r)=g_{\chi \chi}(r) g_{r r}(r)
$$

la entropía de entrelazamiento se puede escribir

$$
\mathcal{S}_{\mathcal{A}}=\frac{2 \pi \Lambda}{\kappa_{(d+1)}^{2}} \int d \zeta \sqrt{\eta^{2}(r) r^{\prime 2}+f^{2}(r) x_{1}^{\prime 2}}
$$

Minimizando (4.34) se obtiene

$$
x_{1}^{\prime}(\zeta)= \pm \frac{f\left(r_{0}\right) \eta(r)}{f(r)} \frac{r^{\prime}(\zeta)}{\sqrt{f^{2}(r)-f^{2}\left(r_{0}\right)}}
$$

en donde $r=r_{0}$ es el valor mínimo en la coordenada holográfica alcanzada por la superficie. Dependiendo del fondo gravitatorio estudiado este punto podría ser el radio del horizonte o el fin del espacio-tiempo. Invirtiendo esta relación se puede leer la longitud de la banda en la dirección $x_{1}$

$$
L=2 \int_{r_{0}}^{\infty} d r \frac{d x_{1}}{d r}=2 \int_{r_{0}}^{\infty} d r \frac{\eta(r)}{f(r)} \frac{f\left(r_{0}\right)}{\sqrt{f^{2}(r)-f^{2}\left(r_{0}\right)}} .
$$

Ahora se fijará la invarianza ante difeomorfismo como $x_{1}(\zeta)=\zeta$, esta elección tiene la ventaja de proveer una parametrización completa de $r\left(x_{1}\right),\left(x_{1} \in\left[-\frac{L}{2}, \frac{L}{2}\right]\right.$ y las condiciones de borde son $\left.r\left( \pm \frac{L}{2}\right)=\infty\right)$. A partir de (4.35) en (4.34) la entropía de entrelazamiento queda

$$
\mathcal{S}_{\mathcal{A}}\left(r_{0}\right)=2 \frac{2 \pi \Lambda}{\kappa_{(d+1)}^{2}} \int_{r_{0}}^{\infty} d r \frac{f(r) \eta(r)}{\sqrt{f(r)^{2}-f\left(r_{0}\right)^{2}}} .
$$

la expresión (4.37) diverge en $r=\infty$ debido a la extensión infinita de la superficie. Esta divergencia se entiende a partir de la existencia de otra solució, con las mismas condiciones de borde, consistente de dos superficies desconectadas que se expanden todo a lo largo de la dirección radial. Su area es

$$
\mathcal{S}_{\mathcal{A}_{\text {disc }}}=2 \frac{2 \pi \Lambda}{\kappa_{(d+1)}^{2}} \int_{r_{\text {min }}}^{\infty} d r \eta(r),
$$

aquí $r_{\text {min }}$ es el valor mínimo de $r$ permitido por la geometría. La EE queda definida con respecto al estado de referencia (4.38)

$$
\Delta \mathcal{S}_{\mathcal{A}}=\frac{4 \pi \Lambda}{\kappa_{(d+1)}^{2}}\left(\int_{r_{0}}^{\infty} d r \frac{f(r) \eta(r)}{\sqrt{f(r)^{2}-f\left(r_{0}\right)^{2}}}-\int_{r_{\text {min }}}^{\infty} d r \eta(r)\right)
$$

En lo que sigue vamos a estudiar la EE para las soluciones de (4.10) y (4.1.2). 
En el caso del superconductor tipo $p$ las funciones relevantes son

$$
f_{p}^{2}(r)=g_{y y} g_{x x}=r^{4}, \quad \eta_{p}^{2}(r)=g_{y y} g_{r r}=\frac{r^{2}}{h^{2} N}
$$

en donde el subíndice $p$ nos recuerda que corresponden al tipo $p$. Con esto, podemos calcular explícitamente la cantidad (4.39) With this, we can compute explicitly the quantity (4.39)

$$
\Delta \mathcal{S}_{\mathcal{A}}=\frac{4 \pi \Lambda}{\kappa_{(4)}^{2}}\left(\int_{r_{0}}^{\infty} d r \frac{r^{3}}{h \sqrt{N} \sqrt{r^{4}-r_{0}^{4}}}-\int_{r_{\text {min }}}^{\infty} d r \frac{r}{h \sqrt{N}}\right)
$$

En la figura 4.12 se muestra $\Delta \mathcal{S}_{\mathcal{A}}$ como función de la longitud de la banda $L$. Se graficó para diferentes valores del parámetro de deformación y diferentes valores de la temperatura. Como era esperado, la curva inferior es la que tiene menor temperatura debido a que al bajar la temperatura hay más grados de libertad condensados. El comportamiento lineal para valores grandes de $\mu L$ es una manifestación de la ley de area propuesta en (4.30). La figura 4.14 muestra la EE de las fases condensadas (línea azul) y normal (linea roja) como función de la temperatura y para un valor constante de $L$.

Con el objetivo de obtener una entropía finita sin sustraer la solución desconectada, podemos escribir la EE de la siguiente manera

$$
\mathcal{S}_{\mathcal{A}}\left(r_{0}\right)=\frac{4 \pi \Lambda}{\kappa_{(4)}^{2}} \int_{r_{0}}^{\mathcal{R}} d r \frac{r^{3}}{h \sqrt{N} \sqrt{r^{4}-r_{0}^{4}}}=S_{\mathcal{A}}+\frac{4 \pi \Lambda}{\kappa_{(d+1)}^{2}} \mathcal{R}
$$

en donde $S_{\mathcal{A}}$ tiene dimensiones de longitud y no presenta divergencias. La figura 4.14 muestra que la EE para el superconductor (linea azul) es menor que para la solución de RN (linea roja). Como se mencionó anteriormente esto es esperable debido que la solución superconductora tiene grados de libertad condensados.

Realizando el mismo análisis para la solución de (4.1.2) se obtiene

$$
f_{p+i p}^{2}(r)=g_{y y} g_{x x}=r^{4} h^{4}, \quad \eta_{p+i p}^{2}(r)=g_{y y} g_{r r}=\frac{r^{2} h^{2}}{M},
$$

y la siguiente EE

$$
\Delta \mathcal{S}_{\mathcal{A}}=\frac{4 \pi \Lambda}{\kappa_{(4)}^{2}}\left(\int_{r_{0}}^{\infty} d r \frac{r^{3} h^{3}}{\sqrt{M} \sqrt{r^{4} h^{4}-r_{0}^{4} h\left(r_{0}\right)^{4}}}-\int_{r_{\text {min }}}^{\infty} d r \frac{r h}{\sqrt{M}}\right)
$$

La figura 4.13 muestra el comportamiento de $\Delta \mathcal{S}_{\mathcal{A}}$ para este caso, y podemos realizar el mismo análisis que para el caso del superconductor $p$. De nuevo, podemos utilizar una aproximación diferente para obtener la entropía no divergente sin realizar la sustracción de las soluciones desconectadas. Esto consiste en separar la parte divergente de la integral (4.37) y tener en cuenta su parte finita, $S_{\mathcal{A}}$. En este caso

$$
\mathcal{S}_{\mathcal{A}}\left(r_{0}\right)=\frac{4 \pi \Lambda}{\kappa_{(4)}^{2}} \int_{r_{0}}^{\mathcal{R}} d r \frac{r^{3} h^{3}}{\sqrt{M} \sqrt{r^{4} h^{4}-r_{0}^{4} h\left(r_{0}\right)^{4}}}=S_{\mathcal{A}}+\frac{4 \pi \Lambda}{\kappa_{(4)}^{2}} \mathcal{R} .
$$




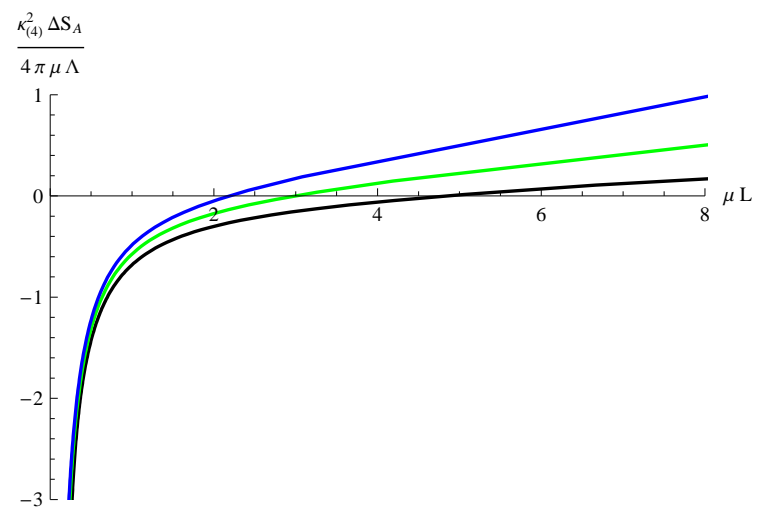

Figura 4.12: Entropía de entrelazamiento como función del tamaño de la banda para la solución tipo $p$. Las curvas negra, verde y azul corresponden a $g_{Y M}=1, T=0,0749 \mu, g_{Y M}=$ $1,5, T=0,1565 \mu$ у $g_{Y M}=2, T=0,2312 \mu$ respectivamente.

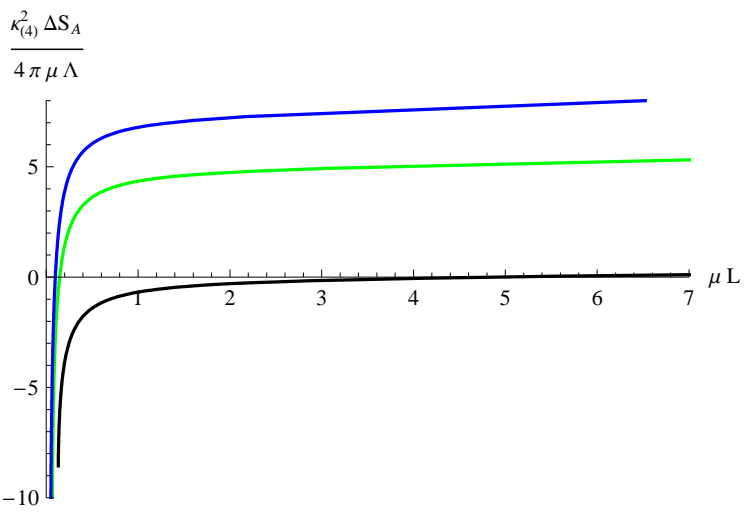

Figura 4.13: Entropía de entrelazamiento como función del tamaño de la banda para la solución tipo $p+i p$. Las curvas negra, verde y azul corresponden a $g_{Y M}=1, T=0,0749 \mu$, $g_{Y M}=1,5, T=0,1565 \mu$ у $g_{Y M}=2, T=$ $0,2312 \mu$ respectivamente.

La figura 4.14 muestra esta parte finita (linea naranja) para $g_{Y M}=2$ y $T_{c}=0,2312$, de donde se ve que, como se esperaba, es menor que la EE de la solución de RN. Además la figura muestra que la EE en el caso del superconductor tipo $p$ es menor que para el $p+i p$. Esto sugiere que a una dada temperatura hay más grados de libertad condensados en el superconductor $p$.

\subsubsection{Conclusiones}

En este capítulo se estudiaron los duales holográficos a superconductores tipo $p$ y $p+i p$ en $3+1$ dimensiones. Se calculó la deformación de la geometría dual al tipo $p$ y sus propiedades termodinámicas. Como era esperable, y en contraste a la solución en $4+1$ dimensiones de [140], se encontró una transición de fase de segundo orden entre las fases normal y superconductora. Luego, se resumió la deformación producida por el campo de gauge sobre la geometría del $p+i p$ y se comparó con nuestra solución. A partir del estudio de las cantidades termodinámicas y en particular de sus potencial gran canónico, se notó que para un valor fijo de la temperatura la solución tipo $p$ tiene menor potencial y por lo tanto es la preferida por el sistema. Esto se relaciona con el echo de que la solución tipo $p+i p$ es inestable frente a pequeñas fluctuaciones y decae a la solución tipo $p$.

Finalmente, usando la propuesta holográfica estudiada en [82], se calculó la entropía de entrelazamiento de la teoría cuántica de campos usando su dual gravitatorio, para una geometría de 


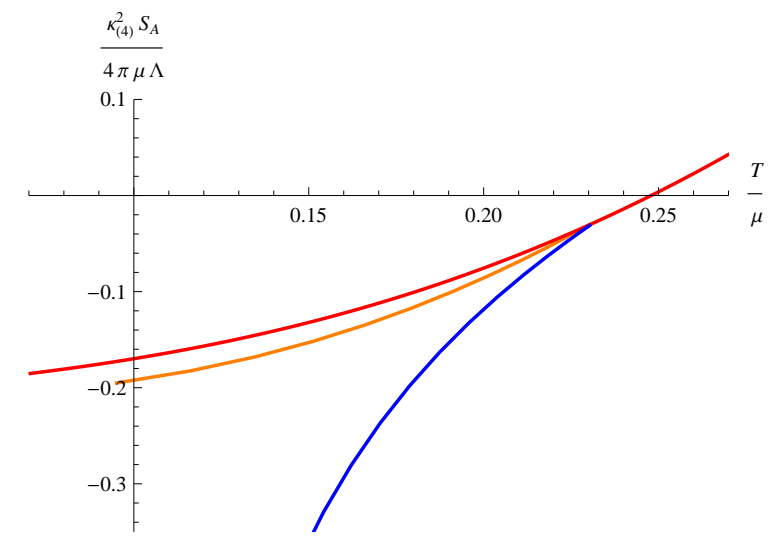

Figura 4.14: Entropía de entrelazamiento como función de la temperatura y a $\mu L=3$ fijo. La curva azul es para la solución tipo $p$, la naranja para la tipo $p+i p$ y la roja para RN. Se utilizó $g_{Y M}=2$ y $T_{c}=0,2312$.

banda y como función de la temperatura y el tamaño de la banda. Se observó que en ambos casos la EE se comporta lineal para valores grandes de $L$, lo cual confirma la ley de area propuesta. La EE vs L tiende a valores mas grandes de $\Delta \mathcal{S}_{\mathcal{A}}$ a medida que se incrementa su temperatura. Como función de la temperatura se observa que la mayor EE es para la solución de RN. Esto era esperable debido a que el superconductor tiene grados de libertad condensados. Además la solución tipo $p$ presenta más grados de libertad condensados que el $p+i p$. Esto podría explicar el echo de que el valor del condensado para el tipo $p$ es más grande que para el $p+i p$ a una dada temperatura. 


\section{Capítulo 5}

\section{Conclusiones}

En esta tesis se estudiaron varias aplicaciones de la conjetura de Maldacena. En particular, a lo largo del trabajo se han analizado desde el punto de vista holográfico (gravitatorio) teorías de campos conformes, no conformes y sistemas de materia condensada. A lo largo de la tesis se desarrollaron distintos aspectos técnicos de la conjetura de Maldacena mostrando cómo se obtienen las funciones de correlación y los valores de expectación del lazo de Wilson de la teoría de gauge dual a partir de cálculos en gravedad. También se estudiaron las recientes aplicaciones de la conjetura al estudio de un sistema superconductor. Los aspéctos estudiados corresponden a cálculos originales que condujeron a publicaciones en revistas internacionales con referato.

En el capítulo 2 se ha extendido la prescripción GKPW para calcular funciones de correlación de teorías de campos duales a fondos de gravedad que poseen dos bordes asintóticamente AdS. En el camino se analizaron las caracterísiticas particulares de la prescripción de GKPW en espaciotiempos de signatura Lorentziana. Se propuso también un método que nos permite concluir si las teorías de campos en dichos bordes interactúan entre sí o se entrelazan.

En el capítulo 3 se estudió el valor de expectación del operador lazo de Wilson para diferentes representaciones del grupo de gauge desde el punto de vista dual gravitatorio. En particular, se analizaron fluctuaciones para diferentes fondos de gravedad duales a teorías de campos no conformes y con supersimetría no maximal. La estabilidad se estudió para el caso del lazo de Wilson rectangular y en la representación fundamental. Asimismo la conjetura provee un marco para estudiar el potencial monopolo-antimonopolo: estos se estudiarón para las geometrías de Maldacena-Nuñez y Klebanov-Strassler, soluciones de gravedad que modelan el régimen IR de $\mathcal{N}=1 \mathrm{SYM}$, con el objeto de chequear las ideas de' $t$ Hooft [2]. Se analizaron fluctuaciones tanto bosónicas como fermiónicas al fondo gravitatorio dual a una línea de Wilson- 't Hooft.

Por último en el capítulo 4 se estudió la termodinámica de un superconductor tipo $p$ en $2+1$ dimensiones, en el límite en el que el número de grados de libertad cargados y el número de grados de libertad totales son comparables, mediante su descripción gravitatoria dual. Se observó que, al igual 
que en el caso del $p+i p$, existe un transición de fase de segundo orden entre un estado normal y uno superconductor al variar la temperatura. Además se concluyó que por debajo de una temperatura crítica la configuración más estable es la solución dual correspondiente al superconductor tipo $p$. Con el objeto de probar la solución, se calculó la entropía de entrelazamiento utilizando la propuesta de Ryu - Takayanagi y se mostró que esta satisface la ley de área. 


\section{Apéndice A}

\section{Lazos de Wilson}

\section{A.1. De Sturm-Liouville a Schrödinger}

Las ecuaciones (3.22)-(3.23) son del tipo de Sturm-Liouville

$$
\left[-\frac{d}{d r}\left(P\left(r, r_{0}\right) \frac{d}{d r}\right)+U\left(r, r_{0}\right)\right] \Phi(r)=\omega^{2} Q\left(r, r_{0}\right) \Phi(r), \quad r_{0} \leq r<\infty
$$

las funciones $P\left(r, r_{0}\right)$ y $Q\left(r, r_{0}\right)$ pueden leerse de $(3.22)-(3.23)$, en ambos casos $U\left(r, r_{0}\right)=0$. El cambio de variables

$$
y=\int_{r_{0}}^{r} \sqrt{\frac{Q}{P}} d r, \quad \Phi(r)=(P Q)^{-\frac{1}{4}} \Psi(y)
$$

transforma (A.1) en una ecuación del tipo Schrödinger

$$
\left[-\frac{d^{2}}{d y^{2}}+V\right] \Psi=\omega^{2} \Psi, \quad 0 \leq y \leq y_{0} .
$$

Aquí $y_{0}=\int_{r_{0}}^{\infty} d r \sqrt{\frac{Q}{P}}$ el cual puede ser infinito o finito dependiendo de la naturaleza de $Q, P$ y se puede chequear que (A.2) es integrable en el límite inferior dado por $y \sim \sqrt{r-r_{0}}$. El potencial $V$ esta dado por

$$
\begin{aligned}
V & =\frac{U}{Q}+\left[(P Q)^{-\frac{1}{4}} \frac{d^{2}}{d y^{2}}\right](P Q)^{\frac{1}{4}} \\
& =\frac{U}{Q}+\left[\frac{P^{\frac{1}{4}}}{Q^{\frac{3}{4}}} \frac{d}{d r}\left(\sqrt{\frac{P}{Q}} \frac{d}{d r}\right)\right](P Q)^{\frac{1}{4}}
\end{aligned}
$$

Los puntos $r=r_{0}$ y $r=\infty$ se mapean a $y=0$ e $y=y_{0}$ respectivamente. Las condiciones de borde que deben imponerse en la solución de (A.3) son [99]:

- Infinito: extremos de la cuerda fijos ${ }^{1}$

$$
\left.\delta x\right|_{r=\infty}=\left.0 \Rightarrow \Psi\right|_{y=y_{0}}=0
$$

\footnotetext{
${ }^{1}$ Ver, sin embargo, un detalle en el contexto de Maldacena-Núñez (secc. 3.4.3) cuando se impone (A.5).
} 
- Tip $r=r_{0}$ : para fluctuaciones en el plano, $\delta x_{1}$, y fluctuaciones transversales $\delta x_{m}$ se obtiene a partir de (3.24),(3.31),(3.32)

$$
\begin{gathered}
\text { Soluciones pares: }\left.\frac{d \Psi}{d y}\right|_{y=0}=0 \\
\text { Soluciones impares: }\left.\Psi\right|_{y=0}=0 .
\end{gathered}
$$

\section{A.2. Espectro para fluctuaciones transversales en $A d S_{5} \times S^{5}$}

En esta sección se repetirá la solución de [97] para el espectro exacto de las fluctuaciones longitudinales en el fondo de gravedad $A d S_{5} \times S^{5}$ y se comparará con los valores obtenidos numéricamente en esta tesis descriptos en el final de la sección 3.1.2. La métrica de $A d S$ en coordenadas de Poincare se escribe

$$
d s^{2}=\frac{R^{2}}{z^{2}}\left(-d t^{2}+d x_{i} d x_{i}+d z^{2}\right)+R^{2} d \Omega_{5}^{2} .
$$

El fijado de gauge $x t=\tau, x=x_{\mathrm{cl}}, z=z_{\mathrm{cl}}(x)$ conduce a

$$
\left(\frac{d z_{\mathrm{cl}}}{d x}\right)^{2}=\frac{z_{0}^{4}-\left(z_{\mathrm{cl}}\right)^{4}}{\left(z_{\mathrm{cl}}\right)^{4}}
$$

La solución a (A.8) con los extremos de la cuerda separados por una distancia $L$ es (3.34)-(3.35).

$$
x_{\mathrm{cl}}(z)= \pm z_{0}\left[\frac{(2 \pi)^{\frac{3}{2}}}{2 \Gamma\left[\frac{1}{4}\right]^{2}}-\frac{1}{4} \mathrm{~B}\left(\frac{z^{4}}{z_{0}^{4}} ; \frac{3}{4}, \frac{1}{2}\right)\right]
$$

en donde $z_{0}=z_{\mathrm{cl}}(0)=\left(\Gamma\left[\frac{1}{4}\right]^{2} /(2 \pi)^{\frac{3}{2}}\right) L$ es la distancia radial máxima alcanzada por la cuerda (tip de la cuerda) y $\mathrm{B}(z ; a, b)$ es la función beta incompleta $\mathrm{B}(z ; a, b)=\int_{0}^{z} t^{a-1}(1-t)^{b-1} d t$.

Las fluctuaciones alrededor de la solución (A.9) en las coordenadas transversales $x_{m}(m=2,3)$ son desacopladas, escribiendo $X^{\mu}=\left(t, x_{\mathrm{cl}}(\sigma), \delta x_{m}(t, \sigma), z_{\mathrm{cl}}(\sigma)\right)$ las ecuaciones a orden lineal son [97],[104]

$$
\begin{array}{ll}
x \text {-gauge : } & {\left[\partial_{t}^{2}-\frac{z_{\mathrm{cl}}^{4}(x)}{z_{0}^{4}} \partial_{x}^{2}\right] \delta x_{m}(t, x)=0} \\
r \text {-gauge : } & {\left[\partial_{t}^{2}-\left(1-\frac{z^{4}}{z_{0}^{4}}\right) \partial_{z}^{2}+\frac{2}{z} \partial_{z}\right] \delta x_{m}(t, z)=0 \quad m=2,3 .}
\end{array}
$$

Como se ha mencionado en la sección 3.1, nótese que la ecuación de movimiento en el gauge $x$ (A.10) depende explícitamente de la solución clásica $z_{\mathrm{cl}}(x)$. Las ecuaciones se relacionan mediante el cambio de variables en (A.9). Escribiendo $\delta x_{m}=e^{-i w t} f(z)$ en (A.11) y nombrando $\tilde{z}=z / z_{0}$ se obtiene [104]

$$
\left[\left(1-\tilde{z}^{4}\right) \partial_{\tilde{z}}^{2}-\frac{2}{\tilde{z}}+\xi^{2}\right] f(\tilde{z})=0, \quad 0 \leq \tilde{z} \leq 1,
$$

en donde $\xi=z_{0} \omega$. El cambio de variables [138]

$$
\begin{aligned}
& f(\tilde{z})=\sqrt{1+\xi^{2} \tilde{z}^{2}} F(q) \\
& q(\tilde{z})= \pm 2 \int_{\tilde{z}}^{1} \frac{t^{2}}{\left(1+(\xi t)^{2}\right) \sqrt{1-t^{4}}} d t
\end{aligned}
$$


transforma la ecuación (A.12) en un oscilador armónico simple

$$
\frac{d^{2} F}{d q}+\frac{1}{4} \xi^{2}\left(\xi^{4}-1\right) F=0, \quad q \in\left[-q_{*}, q_{*}\right]
$$

en donde $q_{*}=q(0)$. Las condiciones de borde en infinito $\delta x_{m}(t, 0)=0$ se han mapeado en $F\left(q_{*}\right)=$ 0 , y cuantiza las frecuencias en (A.14) conduciendo a

$$
\omega_{n} z_{0} \sqrt{\omega_{n}^{4} z_{0}^{4}-1} \int_{0}^{1} \frac{t^{2} d t}{\left(1+w_{n}^{2} z_{0}^{2}\right) \sqrt{1-t^{4}}}=\frac{n \pi}{2}, \quad n=1,2, \ldots
$$

La tabla siguiente muestra la comparación entre los autovalores exactos (A.15) y el análisis numérico de autovalores de (A.12) con $z_{0}=1$.

\begin{tabular}{|l||c|l|}
\hline & Exact & Numeric \\
\hline$\omega_{1}$ & 2.203 & 2.226 \\
$\omega_{2}$ & 3.467 & 3.492 \\
$\omega_{3}$ & 4.697 & 4.735 \\
$\omega_{4}$ & 5.914 & 5.959 \\
$\omega_{5}$ & 7.125 & 7.181 \\
$\omega_{6}$ & 8.332 & 8.396 \\
$\omega_{7}$ & 9.537 & 9.612 \\
$\omega_{8}$ & 10.741 & 10.823 \\
\hline
\end{tabular}

Los autovalores impares (pares) se obtuvieron resolviendo (A.12) con condiciones de borde impares (pares) discutidas después de (3.24).

\section{A.3. Fluctuaciones fermiónicas del operador de Wilson-'t Hooft}

La matriz $\tilde{M}^{\alpha \beta}$ evaluada en la solución clásica (3.112) toma la forma

$$
\tilde{M}^{\alpha \beta}=\frac{L^{2} \sinh ^{2}\left(u_{e q}\right)}{k^{2}+L^{4} \sinh ^{4}\left(u_{e q}\right)}\left(\begin{array}{cccc}
-r^{2} & \frac{2 r^{2} \sqrt{L^{4} \sinh ^{2}\left(u_{e q}\right)-k^{2}}}{L^{2} \sinh \left(2 u_{e q}\right)} & 0 & 0 \\
-\frac{2 r^{2} \sqrt{L^{4} \sinh ^{2}\left(u_{e q}\right)-k^{2}}}{L^{2} \sinh \left(2 u_{e q}\right)} \tilde{\Gamma} & r^{2} & 0 & 0 \\
0 & 0 & 1 & -\frac{k}{L^{2} \sin \theta \sinh ^{2}\left(u_{e q}\right)} \tilde{\Gamma} \\
0 & 0 & \frac{k}{L^{2} \sin \theta \sinh ^{2}\left(u_{e q}\right)} \tilde{\Gamma} & \frac{1}{\sin ^{2} \theta}
\end{array}\right) .
$$

Nótese que los elementos ubicados en la diagonal de esta matriz son los mismos que lo elementos de la diagonal de la inversa de la métrica deformada (3.115).

Ahora se procederá a calcular paso por paso los diferentes factores involucrados en la acción fermiónica (3.104) para la solución de D3-brana analizada en la sección 3.6.2. 
El pullback de la matrices de Dirac 10-dimensionales es

$$
\begin{aligned}
& \Gamma_{t}=\frac{L \cosh \left(u_{k}\right)}{r} \Gamma_{\underline{0}}, \quad \Gamma_{r}=\frac{L \cosh \left(u_{k}\right)}{r} \Gamma_{\underline{1}}, \\
& \Gamma_{\theta}=L \sinh \left(u_{k}\right) \Gamma_{\underline{2}}, \quad \Gamma_{\phi}=L \sinh \left(u_{k}\right) \sin \theta \Gamma_{\underline{3}}
\end{aligned}
$$

y con estas expresiones se encuentra que

$$
\tilde{M}^{\alpha \beta} \Gamma_{\beta} D_{\alpha}=\frac{L \sinh \left(u_{e q}\right)}{\sqrt{k^{2}+L^{4} \sinh ^{4}\left(u_{e q}\right)}}\left[-r e^{2 R_{e} \tilde{\Gamma}} \Gamma_{\underline{0}} D_{t}+r e^{2 R_{e} \tilde{\Gamma}} \Gamma_{\underline{1}} D_{r}+e^{2 R_{m} \tilde{\Gamma}} \Gamma_{\underline{2}} D_{\theta}+\frac{1}{\sin \theta} e^{2 R_{m} \tilde{\Gamma}} \Gamma_{\underline{\underline{3}}} D_{\phi}\right],
$$

en donde han sido utilizadas las matrices de rotación $R_{e}$ y $R_{m}$ definidas por medio de

$$
R_{e}=-\frac{1}{2} \sinh ^{-1}\left(\sqrt{\frac{L^{4} \sinh ^{2}\left(u_{e q}\right)-k^{2}}{L^{4} \sinh ^{4}\left(u_{e q}\right)+k^{2}}}\right) \Gamma_{01}, \quad R_{m}=\frac{1}{2} \arcsin \left(\frac{k}{\sqrt{L^{4} \sinh ^{4}\left(u_{e q}\right)+k^{2}}}\right) \Gamma_{23} .
$$

Usando los siguientes vielbein para la métrica deformada $\hat{g}$

$$
\begin{aligned}
\hat{e}^{\underline{0}}=\frac{\sqrt{\sinh ^{4}\left(u_{e q}\right) L^{4}+k^{2}}}{L r \sinh \left(u_{e q}\right)} d t, & \hat{e}^{\underline{1}}=\frac{\sqrt{\sinh ^{4}\left(u_{e q}\right) L^{4}+k^{2}}}{L r \sinh \left(u_{e q}\right)} d r, \\
\hat{e}^{\underline{\underline{ }}}=\frac{\sqrt{\sinh ^{4}\left(u_{e q}\right) L^{4}+k^{2}}}{L \sinh \left(u_{e q}\right)} d \theta, & \hat{e}^{\underline{3}}=\frac{\sqrt{\sinh ^{4}\left(u_{e q}\right) L^{4}+k^{2}}}{L \sinh \left(u_{e q}\right)} \sin \theta d \phi,
\end{aligned}
$$

se puede simplificar la expresión (A.16) a

$$
\tilde{M}^{\alpha \beta} \Gamma_{\beta} D_{\alpha}=e^{\mathcal{R} \tilde{\Gamma}}\left[\hat{\Gamma}^{\alpha} e^{\mathcal{R} \tilde{\Gamma}} D_{\alpha} e^{-\mathcal{R} \tilde{\Gamma}}\right] e^{\mathcal{R} \tilde{\Gamma}}
$$

en donde se definió $\mathcal{R}=R_{e}+R_{m}$ y se usó que $\hat{\Gamma}_{\alpha}=\hat{e}^{\underline{\alpha}}{ }_{\alpha} \Gamma_{\underline{\alpha}}$ y $\hat{\Gamma}^{\alpha}=\hat{g}^{\alpha \beta} \hat{\Gamma}_{\beta}$.

El pullback de la derivada covariante 10-dimensional se puede escribir

$$
\nabla_{\alpha} d \sigma^{\alpha}=d+\frac{1}{4} \omega \underline{\mu \nu} \Gamma_{\underline{\mu \nu}}+\frac{1}{4} \omega \underline{i j} \Gamma_{\underline{i j}}+\frac{1}{2} \sinh \left(u_{k}\right) e^{\underline{\mu}} \Gamma_{\underline{\mu 4}}+\frac{1}{2} \cosh \left(u_{k}\right) e^{\underline{i}} \Gamma_{\underline{i 4}} .
$$

Los primero 3 términos forman a la derivada covariante con respecto a la geometría deformada del volumen de mundo (3.115). A esta derivada la llamaremos $\hat{\nabla}$ y se define como $\hat{\nabla}_{\alpha}=\partial_{\alpha}+\frac{1}{4} \hat{w} \underline{\underline{\beta \gamma}} \Gamma_{\underline{\beta \gamma}}$. Los dos términos restantes provienen de la curvatura extrínseca del volumen de mundo. Luego, se encuentra que

$$
e^{\mathcal{R} \tilde{\Gamma}} \nabla_{\alpha} d \sigma^{\alpha} e^{-\mathcal{R} \tilde{\Gamma}}=\hat{\nabla}_{\alpha} d \sigma^{\alpha}+\frac{1}{2} \sinh \left(u_{k}\right) e^{\underline{\mu}} \Gamma_{\underline{\mu 4}} e^{-2 R_{e} \tilde{\Gamma}}+\frac{1}{2} \cosh \left(u_{k}\right) e^{\underline{i}} \Gamma_{\underline{i 4}} e^{-2 R_{m} \tilde{\Gamma}}
$$

y por lo tanto podemos escribir al primer termino de la derivada covariante (3.105) como

$$
\tilde{M}^{\alpha \beta} \Gamma_{\beta} \nabla_{\alpha}=e^{\mathcal{R} \tilde{\Gamma}}\left[\hat{\Gamma}^{\alpha} \hat{\nabla}_{\alpha}+\frac{L \sinh \left(u_{e q}\right)}{\sqrt{L^{4} \sinh ^{4}\left(u_{e q}\right)+k^{2}}} \Gamma_{\underline{4}}\left(\sinh \left(u_{e q}\right) e^{-2 R_{e} \tilde{\Gamma}}+\cosh \left(u_{e q}\right) e^{-2 R_{m} \tilde{\Gamma}}\right)\right] e^{\mathcal{R} \tilde{\Gamma}} .
$$


El segundo termino en (3.105) proviene de la 5-forma de Ramond-Ramond y se puede ver fácilmente que

$$
F_{5} \Gamma_{\alpha} d \sigma^{\alpha} \otimes\left(i \sigma_{2}\right)=-4 \Gamma_{\underline{01234}} \otimes\left(i \sigma_{2}\right)\left(\cosh \left(u_{e q}\right) e^{\underline{\mu}} \Gamma_{\underline{\mu}}+\sinh \left(u_{e q}\right) e^{\underline{i}} \Gamma_{\underline{i}}\right)\left(1+\Gamma^{11}\right) .
$$

Como el espinor $\Theta$ satisface $\Gamma^{11} \Theta=\Theta$ se puede reemplazar $\Gamma^{11}=1$ en esta expresión y obtener $\tilde{M}^{\alpha \beta} \Gamma_{\beta} F_{5} \Gamma_{\alpha} \otimes\left(i \sigma_{2}\right)=-16 e^{\mathcal{R} \tilde{\Gamma}} \Gamma_{\underline{01234}} \otimes\left(i \sigma_{2}\right) \frac{L \sinh \left(u_{e q}\right)}{\sqrt{L^{4} \sinh ^{4}\left(u_{e q}\right)+k^{2}}}\left(\cosh \left(u_{e q}\right) e^{-2 R_{m} \tilde{\Gamma}}+\sinh \left(u_{e q}\right) e^{-2 R_{e} \tilde{\Gamma}}\right) e^{\mathcal{R} \tilde{\Gamma}}$.

A partir de (A.17) y (A.18) se tiene

$\tilde{M}^{\alpha \beta} \Gamma_{\beta} D_{\alpha}=e^{\mathcal{R} \tilde{\Gamma}}\left[\hat{\Gamma}^{\alpha} \hat{\nabla}_{\alpha}+\left(1-\Gamma_{D 3}^{(0)}\right) \frac{L \sinh \left(u_{e q}\right)}{\sqrt{L^{4} \sinh ^{4}\left(u_{e q}\right)+k^{2}}} \Gamma_{\underline{4}}\left(\cosh \left(u_{e q}\right) e^{-2 R_{m} \tilde{\Gamma}}+\sinh \left(u_{e q}\right) e^{-2 R_{e} \tilde{\Gamma}}\right)\right] e^{\mathcal{R} \tilde{\Gamma}}$,

en donde

$$
\Gamma_{D 3}^{(0)}=\Gamma_{\underline{0123}} \otimes\left(i \sigma_{2}\right)
$$

Ahora estudiemos al proyector de simetría $\kappa$ denominado $\Gamma_{D 3}$ y definido en [136] como

$$
\Gamma_{D 3}=-\frac{\epsilon^{\alpha_{1} \alpha_{2} \alpha_{3} \alpha_{4}} \Gamma_{\alpha_{1} \alpha_{2} \alpha_{3} \alpha_{4}}}{(p+1) ! \sqrt{\operatorname{det}(g+F)}} \otimes\left(i \sigma_{2}\right) \times \sum_{q} \Gamma^{\alpha_{1} \cdots \alpha_{2 q}} F_{\alpha_{1} \alpha_{2}} \cdots F_{\alpha_{2 q-1} \alpha_{2 q}} \otimes \frac{\left(\sigma_{3}\right)^{q}}{q ! 2^{q}},
$$

en donde $\epsilon^{0123}=1$. Para nuestro caso se obtiene

$\Gamma_{D 3}=-\Gamma_{D 3}^{(0)} \frac{L^{4} \cosh \left(u_{e q}\right) \sinh ^{3}\left(u_{e q}\right)}{L^{4} \sinh ^{4}\left(u_{e q}\right)+k^{2}}\left[1+\left(\frac{k}{L^{2} \sinh ^{2}\left(u_{e q}\right)} \Gamma_{\underline{23}}-\frac{\sqrt{L^{4} \sinh ^{2}\left(u_{e q}\right)-k^{2}}}{L^{2} \cosh \left(u_{e q}\right) \sinh \left(u_{e q}\right)} \Gamma_{\underline{01}}\right) \otimes \sigma_{3}\right]$.

Actuando sobre el espinor $\Theta$ tenemos

$$
\bar{\Theta} \Gamma_{D 3}=-\bar{\Theta} e^{\mathcal{R} \tilde{\Gamma}} \Gamma_{D 3}^{(0)} e^{-\mathcal{R} \tilde{\Gamma}}
$$

para lo cual se ha usado que $\bar{\Theta} \Gamma^{11}=-\bar{\Theta}$.

Usando estas expresiones, el lagrangiano fermiónico para las fluctuaciones de la solución clásica (3.112) se escribe

$$
\mathcal{L}_{F}=\bar{\Theta} e^{\mathcal{R} \tilde{\Gamma}}\left(1+\Gamma_{D 3}^{(0)}\right)\left[\hat{\Gamma}^{\alpha} \hat{\nabla}_{\alpha}+\left(1-\Gamma_{D 3}^{(0)}\right) \frac{L \sinh \left(u_{e q}\right)}{\sqrt{L^{4} \sinh ^{4}\left(u_{e q}\right)+k^{2}}} \Gamma_{\underline{4}}\left(\cosh \left(u_{e q}\right) e^{-2 R_{m} \tilde{\Gamma}}+\sinh \left(u_{e q}\right) e^{-2 R_{e} \tilde{\Gamma}}\right)\right] e^{\mathcal{R} \tilde{\Gamma}} \Theta .
$$

Nótese que como $\left(\Gamma_{D 3}^{0}\right)^{2}=1$ el segundo término dentro del paréntesis se anula. Redefiniendo $e^{\mathcal{R} \tilde{\Gamma}} \Theta=\Theta^{\prime} \Rightarrow \bar{\Theta} e^{\mathcal{R} \tilde{\Gamma}}=\bar{\Theta}^{\prime}$ se obtiene

$$
\mathcal{L}_{F}=\bar{\Theta}^{\prime}\left(1+\Gamma_{D 3}^{(0)}\right) \hat{\Gamma}^{\alpha} \hat{\nabla}_{\alpha} \Theta^{\prime}
$$

El próximo paso es reducir las componentes del espinor usando la simetría $\kappa$, la cual demanda

$$
\tilde{\Gamma} \Theta^{\prime}=\Theta^{\prime}
$$


y nos dice que podemos fijar a cero la mitad de las componentes de $\Theta$. Renombrando $\Theta^{\prime}=\Theta$ se obtiene la forma final para el Lagrangiano

$$
\bar{\Theta}\left(1-\Gamma_{D 3}\right) \tilde{M}^{\alpha \beta} \Gamma_{\beta} D_{\alpha} \Theta=\bar{\Theta} \hat{\Gamma}^{\alpha} \hat{\nabla}_{\alpha} \Theta .
$$

Con este cálculo se obtiene finalmente que a segundo orden en fluctuaciones, la acción fermiónica toma la siguiente forma

$$
S_{F}^{(2)}=\frac{T_{D 3}}{2}\left(\frac{L^{4} \cosh \left(u_{e q}\right) \sinh ^{3}\left(u_{e q}\right)}{L^{4} \sinh ^{4}\left(u_{e q}\right)+k^{2}}\right) \int d^{4} \sigma \sqrt{\hat{g}} \bar{\Theta} \hat{\Gamma}^{\alpha} \hat{\nabla}_{\alpha} \Theta .
$$




\section{Bibliografía}

[1] J. M. Maldacena, Adv. Theor. Math. Phys. 2 (1998) 231 [Int. J. Theor. Phys. 38 (1999) 1113] [arXiv:hep-th/9711200].

[2] G. 't Hooft, Nucl. Phys. B 72, 461 (1974).

[3] O. Aharony, S. S. Gubser, J. M. Maldacena, H. Ooguri and Y. Oz, Phys. Rept. 323 (2000) 183 , hep-th $/ 9905111$.

[4] S. S. Gubser, I. R. Klebanov and A. M. Polyakov, Phys. Lett. B 428 (1998) 105 [arXiv:hepth/9802109].

[5] E. Witten, Adv. Theor. Math. Phys. 2 (1998) 253 [arXiv:hep-th/9802150].

[6] J. M. Maldacena and C. Nunez, Phys. Rev. Lett. 86 (2001) 588 [arXiv:hep-th/0008001].

[7] I. R. Klebanov and M. J. Strassler, JHEP 0008, 052 (2000) [arXiv:hep-th/0007191].

[8] A. Karch and E. Katz, JHEP 0206 (2002) 043, hep-th/0205236; M. Kruczenski, D. Mateos, R. C. Myers and D. J. Winters, JHEP 0307 (2003) 049, hep-th/0304032.

[9] M. B. Green, J. H. Schwarz and E. Witten Cambridge, Uk: Univ. Pr. ( 1987) 469 P. ( Cambridge Monographs On Mathematical Physics)

[10] M. B. Green, J. H. Schwarz and E. Witten Cambridge, Uk: Univ. Pr. ( 1987) 596 P. ( Cambridge Monographs On Mathematical Physics)

[11] J. Polchinski, Phys. Rev. Lett. 75 (1995) 4724 [hep-th/9510017].

[12] I. R. Klebanov, Class. Quant. Grav. 17 (2000) 1161 [hep-th/9908165].

[13] E. Imeroni, arXiv:hep-th/0312070;

[14] V. Balasubramanian, P. Kraus and A. E. Lawrence, Phys. Rev. D 59, 046003 (1999) hepth/9805171. 
[15] V. Balasubramanian, P. Kraus, A. E. Lawrence and S. P. Trivedi, Phys. Rev. D 59 (1999) 104021, hep-th/9808017.

[16] V. Balasubramanian, S. B. Giddings and A. E. Lawrence, JHEP 9903 (1999) 001, hepth/9902052.

[17] K. Skenderis and B. C. van Rees, Phys. Rev. Lett. 101 (2008) 081601, 0805.0150 [hep-th]; JHEP 0905 (2009) 085, arXiv:0812.2909 [hep-th].

[18] P. Breitenlohner and D. Z. Freedman, Phys. Lett. B 115 (1982) 197, Annals Phys. 144 (1982) 249; L. Mezincescu and P. K. Townsend, Annals Phys. 160 (1985) 406.

[19] E. Witten, Adv. Theor. Math. Phys. 2 (1998) 505 [arXiv:hep-th/9803131].

[20] D.Z. Freedman, S.S. Gubser, K. Pilch and N.P. Warner, Adv. Theor. Math. Phys. 3, 363 (1999), hep-th/9904017.

[21] H. Lin, O. Lunin and J.M. Maldacena, JHEP 0410, 025 (2004), hep-th/0409174.

[22] J. de Boer, L. Maoz and A. Naqvi, hep-th/0407212.

[23] G.J. Galloway, K. Schleich, D. Witt and E. Woolgar, Phys. Lett. B505, 255 (2001), hepth/9912119.

[24] K. Skenderis and B. C. van Rees, arXiv:0912.2090 [hep-th].

[25] J.M. Maldacena and L. Maoz, JHEP 0402, 053 (2004), hep-th/0401024.

[26] N. Arkani-Hamed, J. Orgera and J. Polchinski, JHEP 0712, 018 (2007), 0705.2768 [hep-th].

[27] E. Witten and S. T. Yau, Adv. Theor. Math. Phys. 3 (1999) 1635, hep-th/9910245.

[28] M. l. Cai and G. J. Galloway, Adv. Theor. Math. Phys. 3 (1999) 1769, hep-th/0003046.

[29] A. Buchel, Phys. Rev. D70 (2004) 066004, hep-th/0402174.

[30] D. H. Correa, J. Oliva and R. Troncoso, JHEP 0808 (2008) 081, 0805.1513 [hep-th].

[31] J. M. Maldacena, JHEP 0304 (2003) 021, hep-th/0106112.

[32] E. Barnes, D. Vaman, C. Wu and P. Arnold, 1004.1179 [hep-th].

[33] V. Balasubramanian, A. Naqvi and J. Simon, JHEP 0408 (2004) 023, hep-th/0311237. 
[34] P. Martin and J. Schwinger, Phys. Rev. 115, 1342 (1959); J. Schwinger, Jour. Math. Phys. 2, 407(1961); K.T. Mahanthappa, Phys. Rev. 126, 329 (1962); P.M. Bakshi and K.T. Mahanthappa, Jour. Math. Phys. , 1 and 12 (1963); Keldysh, JETP 47, 1515 (1964); Y. Takahashi and H. Umezawa, Collective Phenomena 2, 55 (1975).

[35] T. Azeyanagi, T. Nishioka and T. Takayanagi, Phys. Rev. D 77 (2008) 064005, 0710.2956 [hep-th].

[36] D. Lovelock, J. Math. Phys. 12, 498 (1971).

[37] G. Dotti, J. Oliva and R. Troncoso, Phys. Rev. D 75 (2007) 024002, hep-th/0607062; G. Dotti, J. Oliva and R. Troncoso, Phys. Rev. D 76 (2007) 064038, 0706.1830 [hep-th].

[38] C. P. Herzog and D. T. Son, JHEP 0303 (2003) 046, [arXiv:hep-th/0212072].

[39] M. Ali, F. Ruiz, C. Saint-Victor and J. F. Vazquez-Poritz, Phys. Rev. D 80 (2009) 046002, 0905.4766 [hep-th].

[40] C. P. Herzog, P. K. Kovtun, D. T. Son, Phys. Rev. D79 (2009) 066002. [arXiv:0809.4870 [hep-th]].

[41] D. Marolf, JHEP 0505 (2005) 042 hep-th/0412032.

[42] K. Skenderis and M. Taylor, JHEP 0206 (2002) 025 [hep-th/0204054].

[43] K. G. Wilson, Phys. Rev. D 10 (1974) 2445.

[44] M. E. Peskin and D. V. Schroeder, Reading, USA: Addison-Wesley (1995) $842 \mathrm{p}$

[45] S. J. Rey and J. T. Yee, Eur. Phys. J. C 22 (2001) 379 [arXiv:hep-th/9803001].

[46] J. M. Maldacena, Phys. Rev. Lett. 80, 4859 (1998) [arXiv:hep-th/9803002].

[47] D. E. Berenstein, R. Corrado, W. Fischler and J. M. Maldacena, Phys. Rev. D 59 (1999) 105023 [hep-th/9809188].

[48] N. Drukker, D. J. Gross and H. Ooguri, Phys. Rev. D 60 (1999) 125006 [hep-th/9904191].

[49] J. K. Erickson, G. W. Semenoff and K. Zarembo, Nucl. Phys. B 582 (2000) 155 [hepth/0003055].

[50] G. W. Semenoff and K. Zarembo, Nucl. Phys. Proc. Suppl. 108 (2002) 106 [hep-th/0202156].

[51] N. Drukker and B. Fiol, JHEP 0502 (2005) 010 [hep-th/0501109]. 
[52] S. Yamaguchi, JHEP 0605 (2006) 037 [hep-th/0603208].

[53] C. G. Callan and J. M. Maldacena, Nucl. Phys. B 513 (1998) 198 [hep-th/9708147].

[54] M. R. Douglas, In *Cargese 1997, Strings, branes and dualities* 267-275 [hep-th/9512077].

[55] S. A. Hartnoll and S. P. Kumar, JHEP 0608 (2006) 026 [hep-th/0605027].

[56] S. A. Hartnoll, P. Kovtun, Phys. Rev. D76 (2007) 066001. [arXiv:0704.1160 [hep-th]].

[57] S. A. Hartnoll, P. K. Kovtun, M. Muller, S. Sachdev, Phys. Rev. B76 (2007) 144502. [arXiv:0706.3215 [cond-mat.str-el]].

[58] S. A. Hartnoll, C. P. Herzog, Phys. Rev. D76 (2007) 106012. [arXiv:0706.3228 [hep-th]].

[59] S. S. Gubser, Phys. Rev. D78 (2008) 065034. [arXiv:0801.2977 [hep-th]].

[60] S. A. Hartnoll, C. P. Herzog, G. T. Horowitz, Phys. Rev. Lett. 101 (2008) 031601. [arXiv:0803.3295 [hep-th]].

[61] T. Albash, C. V. Johnson, JHEP 0809 (2008) 121. [arXiv:0804.3466 [hep-th]].

[62] S. A. Hartnoll, C. P. Herzog, G. T. Horowitz, JHEP 0812 (2008) 015. [arXiv:0810.1563 [hep-th]].

[63] M. Cubrovic, J. Zaanen, K. Schalm, Science 325 (2009) 439-444. [arXiv:0904.1993 [hep-th]].

[64] T. Faulkner, H. Liu, J. McGreevy, D. Vegh, Phys. Rev. D83 (2011) 125002. [arXiv:0907.2694 [hep-th]].

[65] H. Liu, J. McGreevy, D. Vegh, Phys. Rev. D83 (2011) 065029. [arXiv:0903.2477 [hep-th]].

[66] T. Faulkner, N. Iqbal, H. Liu, J. McGreevy, D. Vegh, [arXiv:1003.1728 [hep-th]].

[67] T. Faulkner, N. Iqbal, H. Liu, J. McGreevy, D. Vegh, [arXiv:1101.0597 [hep-th]].

[68] N. Iqbal, H. Liu, M. Mezei, [arXiv:1105.4621 [hep-th]]; N. Iqbal, H. Liu, M. Mezei, [arXiv:1108.0425 [hep-th]].

[69] C. Hoyos-Badajoz, K. Jensen and A. Karch, Phys. Rev. D 82 (2010) 086001 [arXiv:1007.3253 [hep-th]].

[70] M. Ammon and M. Gutperle, arXiv:1204.2217 [hep-th]. 
[71] S. A. Hartnoll, Class. Quant. Grav. 26 (2009) 224002 [arXiv:0903.3246 [hep-th]]; J. McGreevy, Adv. High Energy Phys. 2010 (2010) 723105 [arXiv:0909.0518 [hep-th]]; M. Ammon, Fortsch. Phys. 58 (2010) 1123; M. Kaminski, Lect. Notes Phys. 828 (2011) 349 [arXiv:1002.4886 [hepth]]; N. Iqbal, H. Liu and M. Mezei, arXiv:1110.3814 [hep-th]; S. Sachdev, Ann. Rev. Condensed Matter Phys. 3 (2012) 9 [arXiv:1108.1197 [cond-mat.str-el]]; S. A. Hartnoll, "Horizons, holography and condensed matter," arXiv:1106.4324 [hep-th].

[72] E. D'Hoker and D. Z. Freedman, arXiv:hep-th/0201253.

[73] J. McGreevy, Lectures on String theory, MIT Open Course, http://ocw.mit.edu/courses/physics/8-821-string-theory-fall-2008/lecture-notes/

[74] U. H. Danielsson, E. Keski-Vakkuri and M. Kruczenski, JHEP 9901, 002 (1999), hepth/9812007.

[75] D. T. Son and A. O. Starinets, JHEP 0209, 042 (2002), hep-th/0205051.

[76] C. P. Herzog and D. T. Son, JHEP 0303, 046 (2003), hep-th/0212072.

[77] L. Fidkowski, V. Hubeny, M. Kleban and S. Shenker, JHEP 0402 (2004) 014, hep-th/0306170.

[78] D. Z. Freedman, S. D. Mathur, A. Matusis and L. Rastelli, Nucl. Phys. B 546 (1999) 96, hep-th $/ 9804058$.

[79] S. J. Avis, C. J. Isham and D. Storey, Phys. Rev. D 18, 3565 (1978).

[80] I. R. Klebanov and E. Witten, Nucl. Phys. B 556 (1999) 89 arXiv:hep-th/9905104; E. Witten, arXiv:hep-th/0112258.

[81] S. W. Hawking and G. F. R. Ellis, The Large scale structure of space-time, CUP, 1973.

[82] S. Ryu and T. Takayanagi, Phys. Rev. Lett. 96 (2006) 181602, hep-th/0603001.

[83] S. Ryu and T. Takayanagi, JHEP 0608 (2006) 045, hep-th/0605073.

[84] V. E. Hubeny, M. Rangamani and T. Takayanagi, JHEP 0707 (2007) 062, 0705.0016 [hep-th].

[85] T. Nishioka, S. Ryu and T. Takayanagi, J. Phys. A 42 (2009) 504008, arXiv:0905.0932 [hep-th].

[86] N. Seiberg, hep-th/0601234.

[87] N.J.Cornish and D.N.Spergel, math.DG/9906017

[88] A. M. Polyakov, Nucl. Phys. Proc. Suppl. 68 (1998) 1 [arXiv:hep-th/9711002]. 
[89] A. M. Polyakov, Int. J. Mod. Phys. A 14 (1999) 645 [arXiv:hep-th/9809057].

[90] J. Sonnenschein, arXiv:hep-th/0003032.

[91] Y. Kinar, E. Schreiber and J. Sonnenschein, Nucl. Phys. B 566 (2000) 103 [arXiv:hepth/9811192].

[92] D. J. Gross and H. Ooguri, Phys. Rev. D 58 (1998) 106002 [arXiv:hep-th/9805129].

[93] C. P. Herzog and I. R. Klebanov, Phys. Lett. B 526, 388 (2002) [arXiv:hep-th/0111078].

[94] J. Gomis and F. Passerini, JHEP 0608, 074 (2006) [arXiv:hep-th/0604007];

[95] J. Gomis and F. Passerini, JHEP 0701, 097 (2007) [arXiv:hep-th/0612022].

[96] S. S. Gubser, Phys. Rev. D 74 (2006) 126005 [arXiv:hep-th/0605182].

[97] I. R. Klebanov, J. M. Maldacena and C. B. Thorn, JHEP 0604 (2006) 024 [arXiv:hepth/0602255].

[98] J. J. Friess, S. S. Gubser, G. Michalogiorgakis and S. S. Pufu, JHEP 0704 (2007) 079 [arXiv:hep-th/0609137].

[99] S. D. Avramis, K. Sfetsos and K. Siampos, Nucl. Phys. B 769, 44 (2007) [arXiv:hepth/0612139].

[100] S. D. Avramis, K. Sfetsos and K. Siampos, Nucl. Phys. B 793 (2008) 1 [arXiv:0706.2655 [hep-th]].

[101] K. Sfetsos and K. Siampos, JHEP 0808 (2008) 071 [arXiv:0807.0236 [hep-th]].

[102] S. J. Rey, S. Theisen and J. T. Yee, Nucl. Phys. B 527 (1998) 171 [arXiv:hep-th/9803135].

[103] A. Brandhuber, N. Itzhaki, J. Sonnenschein and S. Yankielowicz, Phys. Lett. B 434 (1998) 36 [arXiv:hep-th/9803137].

[104] C. G. Callan and A. Guijosa, Nucl. Phys. B 565 (2000) 157 [arXiv:hep-th/9906153].

[105] Y. Kinar, E. Schreiber, J. Sonnenschein and N. Weiss, Nucl. Phys. B 583 (2000) 76 [arXiv:hep-th/9911123].

[106] S. Forste, D. Ghoshal and S. Theisen, JHEP 9908 (1999) 013 [arXiv:hep-th/9903042].

[107] N. Drukker, D. J. Gross and A. A. Tseytlin, JHEP 0004 (2000) 021 [arXiv:hep-th/0001204]. 
[108] P. C. Argyres, M. Edalati and J. F. Vazquez-Poritz, JHEP 0701 (2007) 105 [arXiv:hepth/0608118].

[109] A. Brandhuber and K. Sfetsos, Adv. Theor. Math. Phys. 3 (1999) 851 [arXiv:hep-th/9906201].

[110] F. Bigazzi, A. L. Cotrone, C. Nunez and A. Paredes, Phys. Rev. D 78 (2008) 114012 [arXiv:0806.1741 [hep-th]].

[111] F. Bigazzi, A. L. Cotrone, A. Paredes and A. V. Ramallo, JHEP 0903 (2009) 153 [arXiv:0812.3399 [hep-th]].

[112] C. Bachas, Phys. Rev. D 33 (1986) 2723.

[113] A. Loewy and J. Sonnenschein, JHEP 0108 (2001) 007 [arXiv:hep-th/0103163].

[114] R. Casero, C. Nunez and A. Paredes, Phys. Rev. D 73 (2006) 086005 [arXiv:hep-th/0602027].

[115] R. Casero, C. Nunez and A. Paredes, Phys. Rev. D 77 (2008) 046003 [arXiv:0709.3421 [hepth]].

[116] C. Hoyos-Badajoz, C. Nunez and I. Papadimitriou, Phys. Rev. D 78 (2008) 086005 [arXiv:0807.3039 [hep-th]].

[117] C. Vafa, J. Math. Phys. 42 (2001) 2798 [arXiv:hep-th/0008142].

[118] M. Bertolini, Int. J. Mod. Phys. A 18, 5647 (2003) [arXiv:hep-th/0303160];

[119] A. Paredes, arXiv:hep-th/0407013.

[120] A. H. Chamseddine and M. S. Volkov, Phys. Rev. Lett. 79 (1997) 3343 [hep-th/9707176].

[121] A. H. Chamseddine and M. S. Volkov, Phys. Rev. D 57 (1998) 6242 [hep-th/9711181].

[122] C. Nunez, M. Piai and A. Rago, arXiv:0909.0748 [hep-th].

[123] M. J. Strassler, arXiv:hep-th/0505153.

[124] G. 't Hooft, Nucl. Phys. B 138 (1978) 1.

[125] M. Bertolini and P. Merlatti, Phys. Lett. B 556 (2003) 80 [arXiv:hep-th/0211142].

[126] C. P. Herzog, I. R. Klebanov and P. Ouyang, arXiv:hep-th/0108101.

[127] A. Kapustin, Phys. Rev. D 74 (2006) 025005 [hep-th/0501015].

[128] B. Chen and W. He, Phys. Rev. D 74 (2006) 126008 [hep-th/0607024]. 
[129] M. T. Grisaru, R. C. Myers and O. Tafjord, JHEP 0008 (2000) 040 [hep-th/0008015].

[130] J. McGreevy, L. Susskind and N. Toumbas, JHEP 0006 (2000) 008 [hep-th/0003075].

[131] R. C. Myers, JHEP 9912 (1999) 022 [hep-th/9910053].

[132] J. Polchinski, Cambridge, UK: Univ. Pr. (1998) 402 p; Cambridge, UK: Univ. Pr. (1998) 531 $\mathrm{p}$

[133] C. V. Johnson, hep-th/0007170.

[134] M. B. Green and J. H. Schwarz, Phys. Lett. B 136 (1984) 367.

[135] J. Simon, Living Rev. Rel. 15 (2012) 3 [arXiv:1110.2422 [hep-th]].

[136] L. Martucci, J. Rosseel, D. Van den Bleeken and A. Van Proeyen, Class. Quant. Grav. 22 (2005) 2745 [hep-th/0504041].

[137] A. Faraggi and L. A. Pando Zayas, JHEP 1105(2011) 018 [arXiv:1101.5145 [hep-th]].

[138] R. C. Brower, C. I. Tan and C. B. Thorn, Phys. Rev. D 73 (2006) 124037 [arXiv:hepth/0603256].

[139] S. S. Gubser and S. S. Pufu, JHEP 0811 (2008) 033 [arXiv:0805.2960 [hep-th]].

[140] M. Ammon, J. Erdmenger, V. Grass, P. Kerner and A. O'Bannon, Phys. Lett. B 686 (2010) 192 [arXiv:0912.3515 [hep-th]].

[141] R.-G. Cai, Z.-Y. Nie and H.-Q. Zhang, Phys. Rev. D 83 (2011) 066013 [arXiv:1012.5559 [hep-th]].

[142] S. S. Gubser, Phys. Rev. Lett. 101 (2008) 191601 [arXiv:0803.3483 [hep-th]].

[143] T. Takayanagi, Class. Quant. Grav. 29 (2012) 153001 [arXiv:1204.2450 [gr-qc]].

[144] T. Albash and C. V. Johnson, JHEP 1205 (2012) 079 [arXiv:1202.2605 [hep-th]].

[145] R.-G. Cai, S. He, L. Li and Y.-L. Zhang, JHEP 1207 (2012) 088 [arXiv:1203.6620 [hep-th]].

[146] R.-G. Cai, S. He, L. Li and Y.-L. Zhang, JHEP 1207 (2012) 027 [arXiv:1204.5962 [hep-th]].

[147] R.-G. Cai, S. He, L. Li and L.-F. Li, arXiv:1209.1019 [hep-th].

[148] M. Ammon, J. Erdmenger, M. Kaminski and P. Kerner, Phys. Lett. B 680 (2009) 516 [arXiv:0810.2316 [hep-th]]. 
[149] P. Basu, J. He, A. Mukherjee and H.-H. Shieh, JHEP 0911 (2009) 070 [arXiv:0810.3970 [hep-th]].

[150] M. Ammon, J. Erdmenger, M. Kaminski and P. Kerner, JHEP 0910 (2009) 067 [arXiv:0903.1864 [hep-th]].

[151] K. Peeters, J. Powell and M. Zamaklar, JHEP 0909 (2009) 101 [arXiv:0907.1508 [hep-th]].

[152] M. Ammon, Fortsch. Phys. 58 (2010) 1123.

[153] M. Kaminski, Lect. Notes Phys. 828 (2011) 349 [arXiv:1002.4886 [hep-th]].

[154] S. A. Hartnoll, arXiv:1106.4324 [hep-th].

[155] S. A. Hartnoll, Class. Quant. Grav. 26 (2009) 224002 [arXiv:0903.3246 [hep-th]].

[156] H. Casini, M. Huerta and R. C. Myers, JHEP 1105 (2011) 036 [arXiv:1102.0440 [hep-th]].

[157] S. Gangopadhyay and D. Roychowdhury, JHEP 1208 (2012) 104 [arXiv:1207.5605 [hep-th]].

[158] E. Winstanley, Lect. Notes Phys. 769 (2009) 49 [arXiv:0801.0527 [gr-qc]].

[159] R. Manvelyan, E. Radu and D. H. Tchrakian, Phys. Lett. B 677 (2009) 79 [arXiv:0812.3531 [hep-th]].

[160] I. R. Klebanov and E. Witten, Nucl. Phys. B 556 (1999) 89 [hep-th/9905104].

[161] I. R. Klebanov, D. Kutasov and A. Murugan, Nucl. Phys. B 796 (2008) 274 [arXiv:0709.2140 [hep-th]].

[162] I. Bah, A. Faraggi, L. A. Pando Zayas and C. A. Terrero-Escalante, Int. J. Mod. Phys. A 24 (2009) 2703 [arXiv:0710.5483 [hep-th]]. 